Portland State University

PDXScholar

7-17-2020

\title{
Exploring Policies and Strategies for the Diffusion of Remote Patient Monitoring (RPM) for the Care of Senior Population
}

Hamad Asri Alanazi

Portland State University

Follow this and additional works at: https://pdxscholar.library.pdx.edu/open_access_etds

Part of the Engineering Commons

Let us know how access to this document benefits you.

\section{Recommended Citation}

Alanazi, Hamad Asri, "Exploring Policies and Strategies for the Diffusion of Remote Patient Monitoring (RPM) for the Care of Senior Population" (2020). Dissertations and Theses. Paper 5505.

https://doi.org/10.15760/etd.7379

This Dissertation is brought to you for free and open access. It has been accepted for inclusion in Dissertations and Theses by an authorized administrator of PDXScholar. Please contact us if we can make this document more accessible: pdxscholar@pdx.edu. 


\title{
Exploring Policies and Strategies for the Diffusion of Remote Patient Monitoring (RPM) for the Care of Senior Population
}

\author{
by \\ Hamad Asri Alanazi
}

A dissertation submitted in partial fulfillment of the requirements for the degree of

\author{
Doctor of Philosophy \\ in \\ Technology Management
}

Dissertation Committee:

Tugrul U. Daim, Chair

David Sibell

Ramin Neshati

Robert R. Harmon

Portland State University

2020 


\begin{abstract}
Telemedicine offers great promise in addressing some of the healthcare challenges such as the increase of healthcare expenditure, a growing population and a projected physician shortage; telemedicine is increasingly being included in the discussion of medicine's future. Telemedicine offers the capabilities to deliver healthcare across distances at reduced costs while maintaining or even increasing the quality of treatment and services.
\end{abstract}

The objective of this research is to develop a model to evaluate alternative solutions to increase the adoption of RPM for the senior population. Also, the research provides a systematic approach for a comprehensive assessment of teleconsultation adoption, develop a decision model to assess the effectiveness of telemedicine policy targeted to increase teleconsultation adoption, evaluate and prioritize current teleconsultation adoption barriers and measure their relative contribution to this adoption, and evaluate and prioritize the alternative solutions and measure their relative contribution to this adoption. Criteria used in this assessment depend on four perspectives that are perceived by decision makers as important for the adoption process. These perspectives are: financial, technical, logistical, and cultural. The research implemented the hierarchical decision model (HDM) to construct a generalized adoption assessment framework. The HDM is used in order to measure the teleconsultation adoption barriers limiting its advantages, and to identify the best alternatives to help its diffusion. 
Experts' qualitative judgments were collected and quantified using the pairwise comparison method. The final rankings and effectiveness of alternative solutions with respect to the mission were identified. The results of this research identified financial barriers as the most influential perspective and that standard processes for reimbursement and multi-state license for telemedicine are the two most effective criteria to accomplish that. The research also applied sensitivity analysis and scenario analysis to identify the effect of perspectives on priority changes on determining the most effective alternative for this perspective. The results showed that standard processes for reimbursement and multi-state license for telemedicine were found to be the two most effective alternatives among the alternative solutions. The research model and outcome can serve as a roadmap in the adoption of teleconsultation or any type of telemedicine. Based on the overall research findings, decision makers can apply specific alternative instruments to support adoption efforts for any given scenario and perspectives emphasis. 


\section{Dedication}

The memory of my parents

To my brothers Salem Farhan, and Mohammed; and my four sisters who believed in me and encouraged me without you all, I would never be here.

To my wonderful wife, Nadiyah, who stood by my side for bad and for good and supported me.

To my lovely children, Abdulaziz, Abdulrahman, Watan, Saud, and Lina, who were the perfect children that anyone could wish for. 


\section{Acknowledgements}

I sincerely acknowledge the many individuals who have provided motivation, encouragement, and guidance throughout this research effort.

I would like to express my gratitude to my advisor, Professor Tugrul U. Daim for his guidance, mentorship, patience, understanding, and encouragement in the challenges of this research process. He has always been there with valuable insights which played a significant role in my Ph.D. journey.

I would like to express my deep appreciation to my dissertation committee members, Dr. David Sibell, Dr. Ramin Neshati, and Dr. Robert R. Harmon for their constructive suggestions and continuous support over the past years as I moved from the research idea to completed research.

I would like to thank all faculty members, staff, and friends in the department of Engineering and Technology Management (ETM) who had been like my second family during the years I spent here. I would like to thank all of the research participants and experts for their time and effort spent on the questionnaires and feedback.

I would like to express my heartfelt gratitude for, my driving force, my brothers Salem, Farhan, and Mohammed; and my four sisters, and without their help, support, and encouragement I will not complete my Ph.D. journey. 
I believe that words not enough to express my great gratitude for the role and help of my lovely wife, Nadiyah during my Ph.D. research. I am especially indebted to her for being extremely understanding, supporting me patiently, and raising our lovely Children, Abdulaziz, Abdulrahman, Watan, Saud, and Lina, without enough help from me during years of my studies and hardship. Her unconditional support fueled my success. 


\section{TABLE OF CONTENTS}

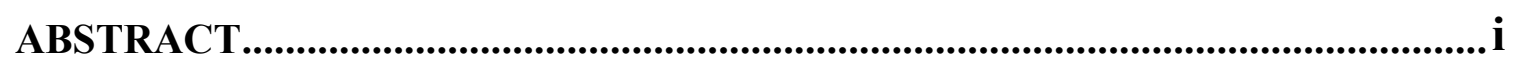

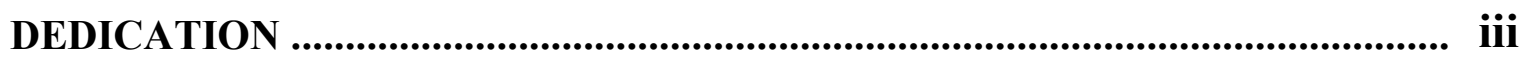

ACKNOWLEDGEMENTS ............................................................................. iv

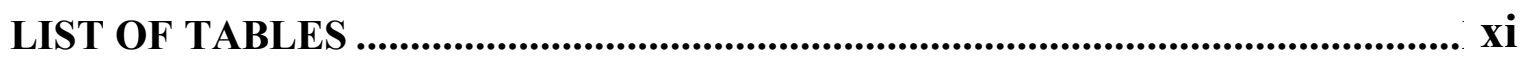

LIST OF FIGURES ......................................................................................... xiii

CHAPTER 1: INTRODUCTION ........................................................................ 1

1.1 The Increase of Health Expenditure .................................................................... 2

1.2 A Growing United States Population ............................................................ 3

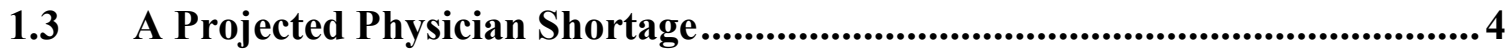

2 CHAPTER 2: LITERATURE REVIEW ................................................ 6

2.1 Health Information Technology (HIT)........................................................ 6

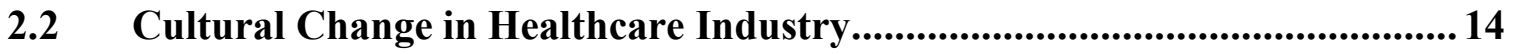

2.3 Telemedicine .................................................................................................... 17

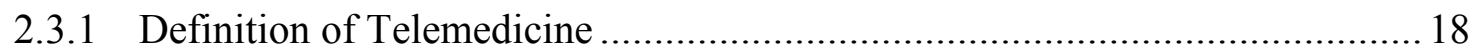

2.3.2 History of Telemedicine ....................................................................... 19

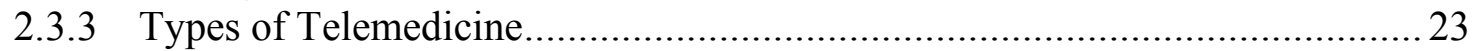

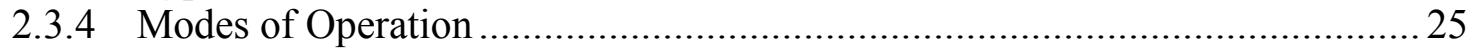

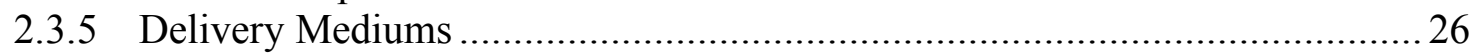

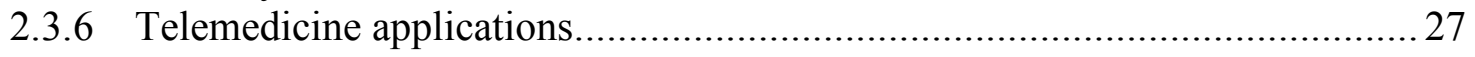

2.4 Teleconsultation ..............................................................................29

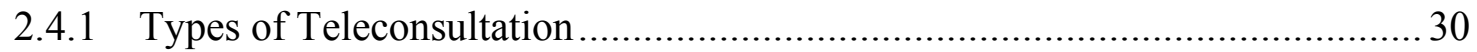

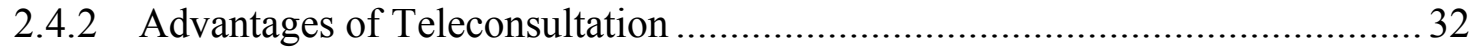

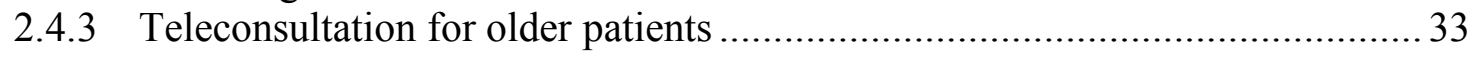

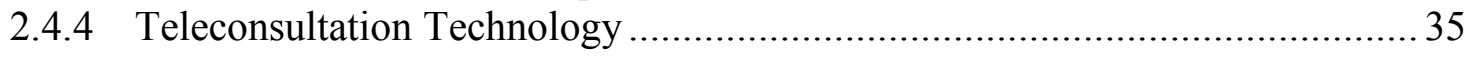

2.4.5 Remote Patient Monitoring (RPM) for older patients ............................... 46

2.5 Successful Telemedicine Applications........................................................50

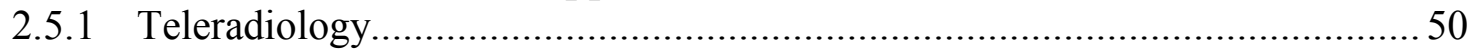

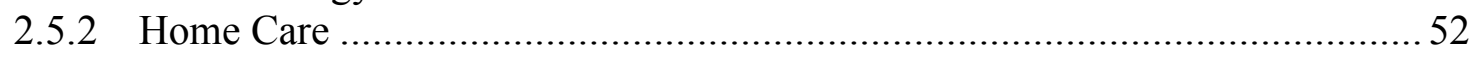




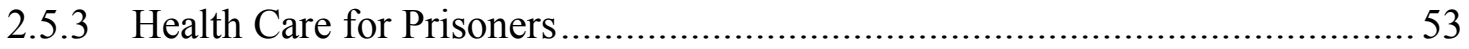

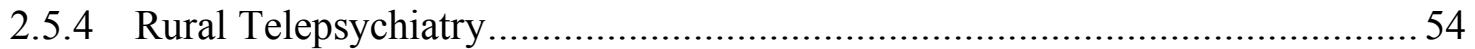

2.5.5 Postsurgical Intensive care in an Urban Nursing Home ................................. 54

2.6 Current Trends in US Telemedicine .................................................................55

2.7 Benefits of Telemedicine..........................................................................61

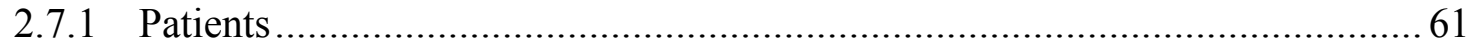

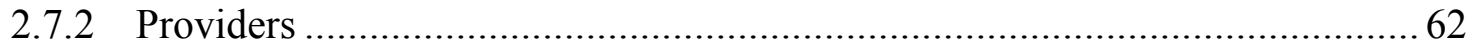

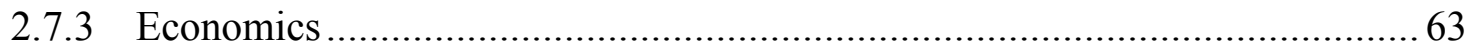

3 CHAPTER 3: RESEARCH APPROACH AND METHODOLOGY ..................65

$3.1 \quad$ Research Objectives ...............................................................................................65

3.2 Research Questions..............................................................................................65

3.3 Multicriteria Decision Making Methods.................................................................67

3.4 Hierarchical Decision Model ................................................................................. 74

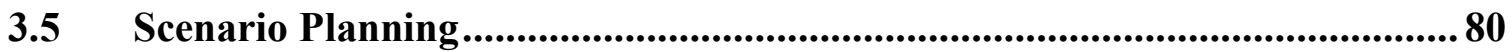

3.5.1 Scenario Planning as a Tool for Strategic Foresight .................................... 83

3.5.2 Strengths and weaknesses of scenarios and scenario analysis ........................ 84

3.5.3 Quantitative Scenario Development Methods................................................ 87

3.5.4 Limitations of Quantitative Scenario Methods .............................................. 87

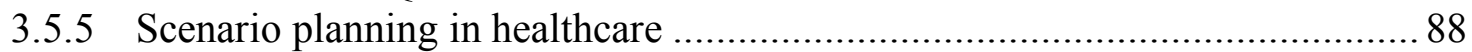

3.5.6 Factors affecting scenario planning and analysis .......................................... 89

3.6 Research Design and Methodology .....................................................................89

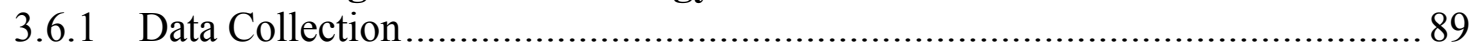

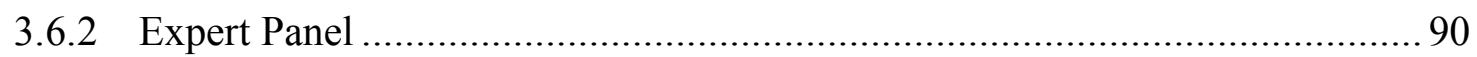

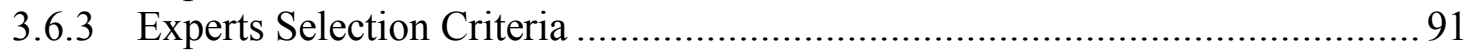

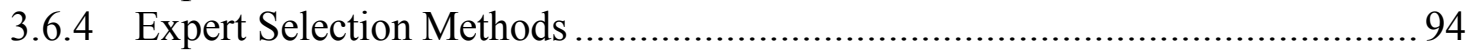

3.7 Research Validity..............................................................................................96

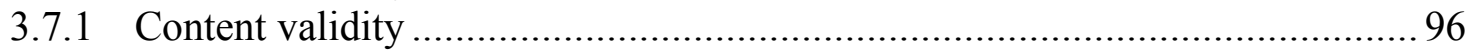

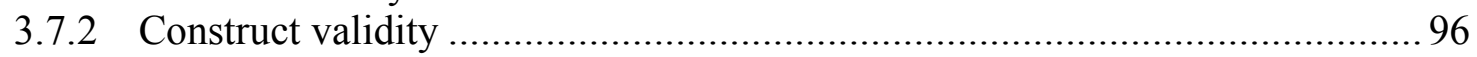

3.8 Data Analysis ........................................................................................................97

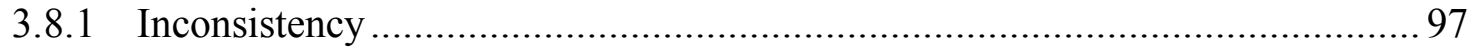

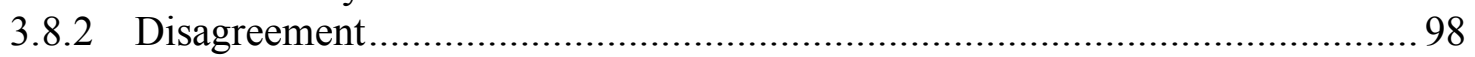

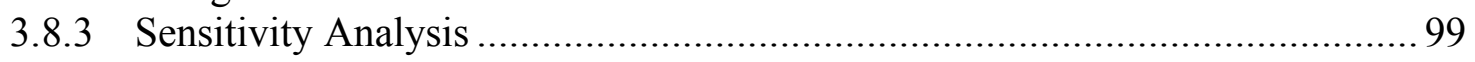

3.9 Research Framework …........................................................................................ 100 
4.1 Adoption Barriers................................................................................................... 101

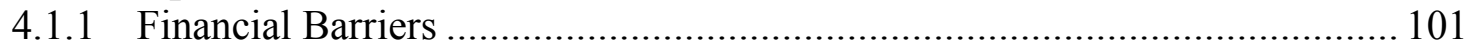

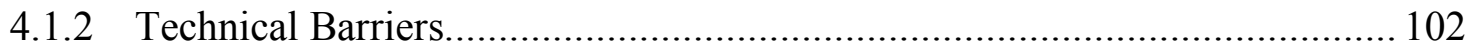

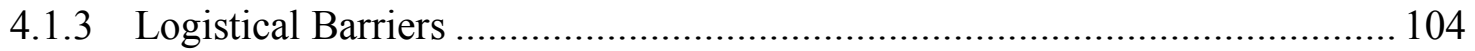

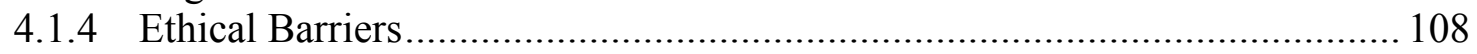

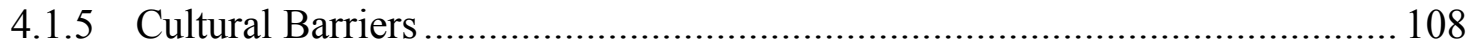

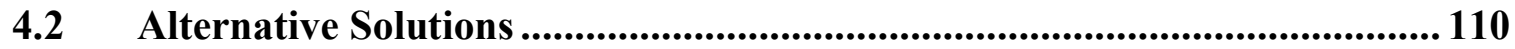

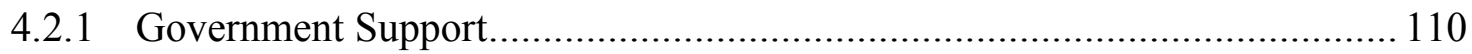

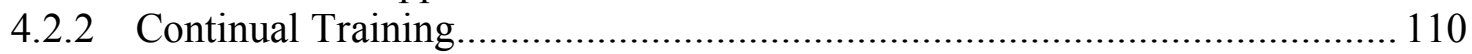

4.2.3 Develop Guidelines for the Use of Telemedicine ....................................... 111

4.2.4 Multi-State Licenses for Telemedicine..................................................... 111

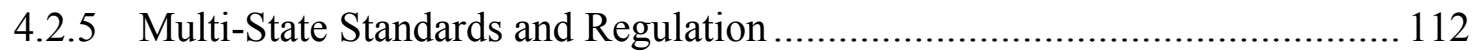

4.2.6 Standard Processes for Reimbursement.................................................. 112

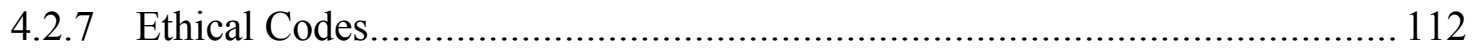

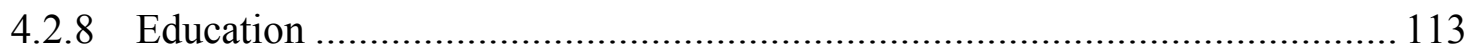

4.3 Adoption Theories ............................................................................................... 113

4.3.1 The Theory of Reasoned Action (TRA) ……............................................. 113

4.3.2 Innovation Diffusion Theory (IDT) .......................................................... 115

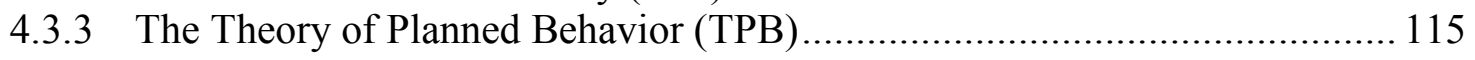

4.3.4 Technology Acceptance Model (TAM) ..................................................... 116

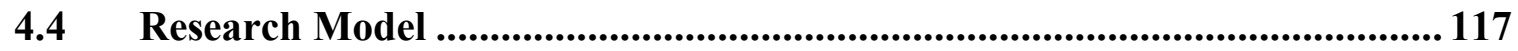

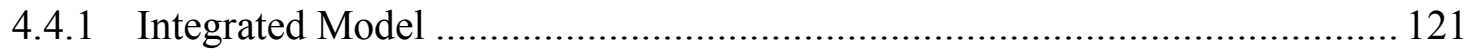

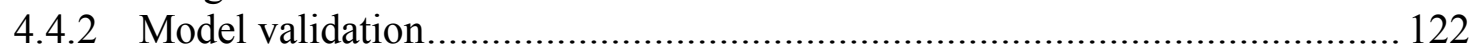

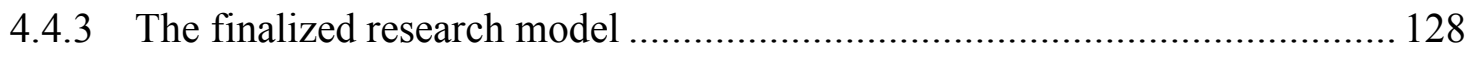

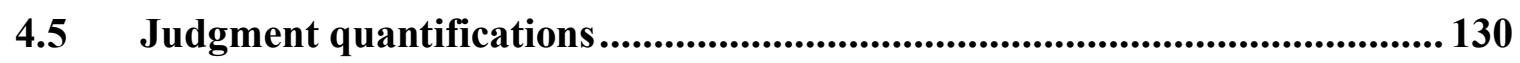

5 CHAPTER 5: DATA ANALYSIS AND RESULTS........................................ 132

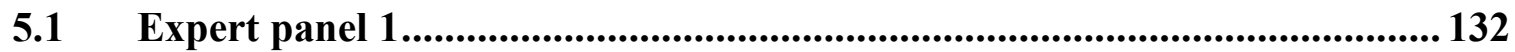

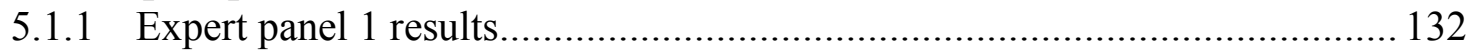

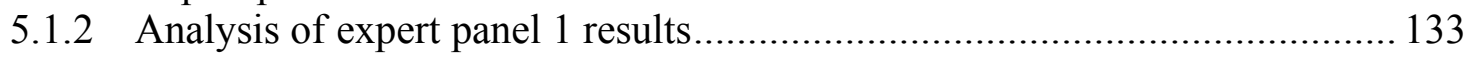

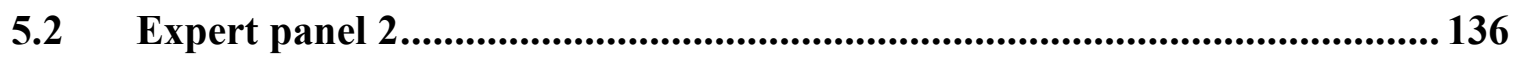

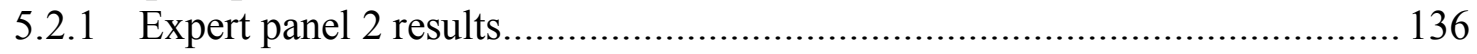

5.2.2 Analysis of expert panel 2 results............................................................. 138

$5.3 \quad$ Expert panel 3............................................................................................. 140

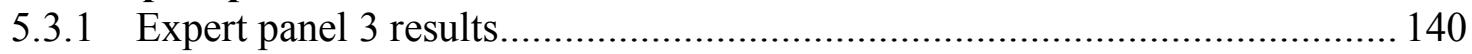

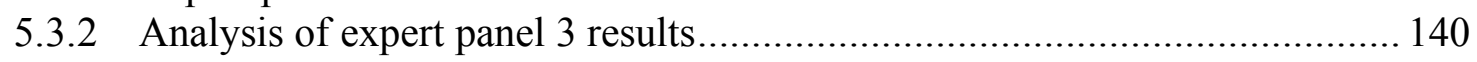




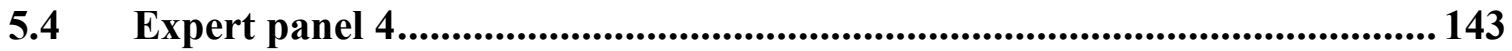

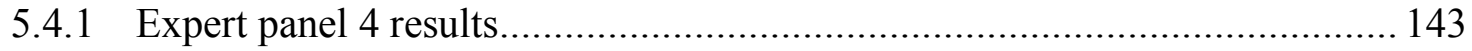

5.4.2 Analysis of expert panel 4 results................................................................. 144

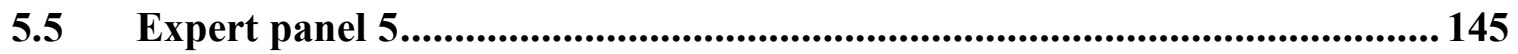

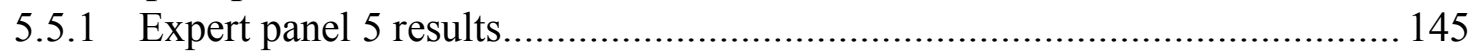

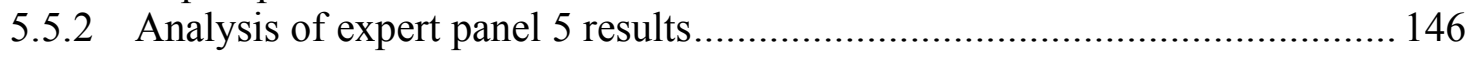

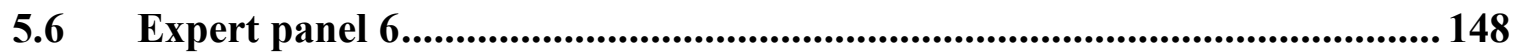

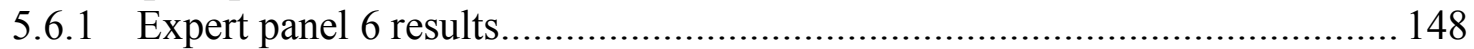

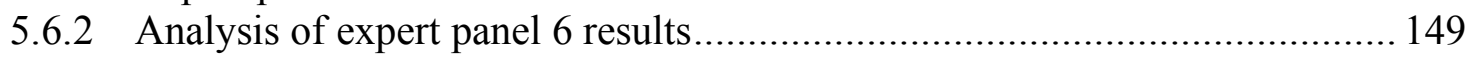

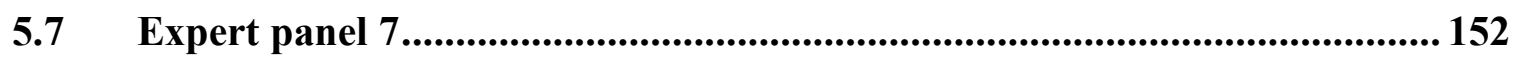

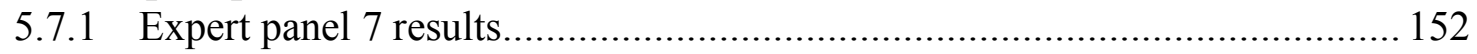

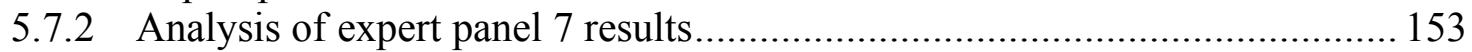

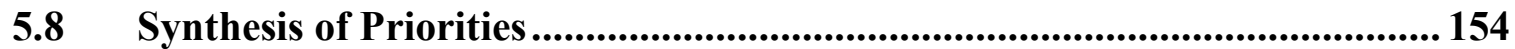

5.8.1 Relative importance of subcriteria with respect to the mission ..................... 154

5.8.2 Overall importance of alternative solutions with respect to the mission........ 156

5.9 Rank analysis of alternatives with respect to expert disagreements............. 158

5.9.1 Analysis of results with respect to expert disagreements in panel 3 .............. 158

5.9.2 Analysis of results with respect to expert disagreements in panel 5 ............. 159

5.9.3 Analysis of results with respect to expert disagreements in panel 6 ............... 161

$5.10 \quad$ Sensitivity Analysis using scenarios approach.................................................. 162

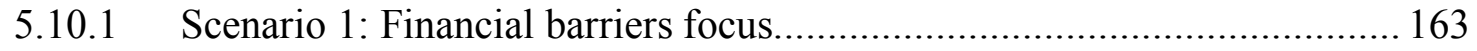

5.10.2 Scenario 2: Technical barriers focus...................................................... 164

5.10.3 Scenario 3: Logistical barriers focus ..................................................... 166

5.10.4 Scenario 4: Cultural barriers focus …………........................................ 167

5.11 Criterion-Related Validity …………………....................................................... 168

6 CHAPTER 6: DISCUSSION ............................................................................ 169

6.1 Technology Acceptance Model (TAM) …………………………........................ 169

6.2 Main perspective adoption barriers...................................................................... 170

$6.3 \quad$ Subcriteria adoption barriers ............................................................................. 172

$6.4 \quad$ Alternative solutions............................................................................................... 174

6.5 Recommendations....................................................................................... 176

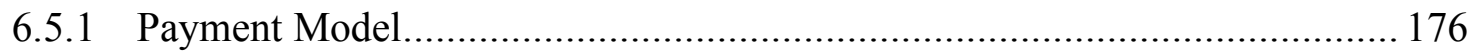

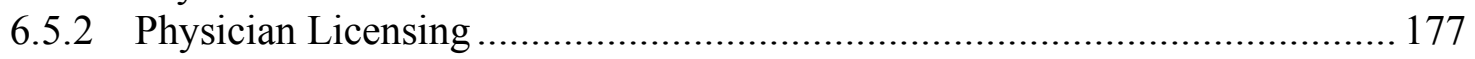

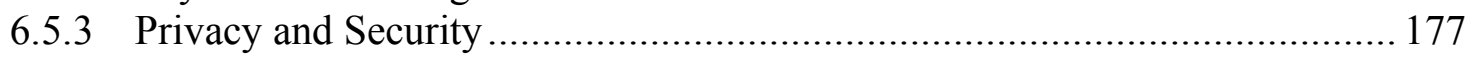


7 CHAPTER 7: CONCLUSIONS, LIMITATIONS AND FUTURE RESEARCH 179

7.1 Research Conclusions and Contributions...................................................179

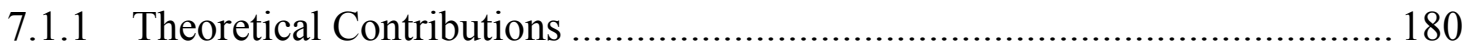

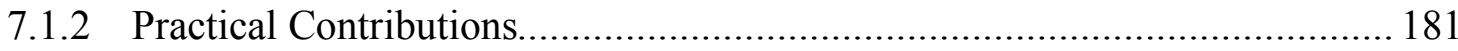

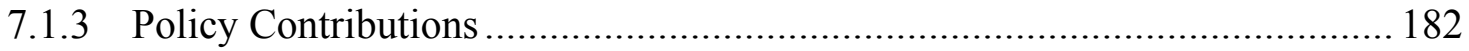

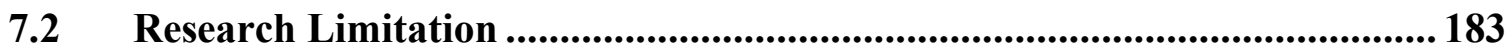

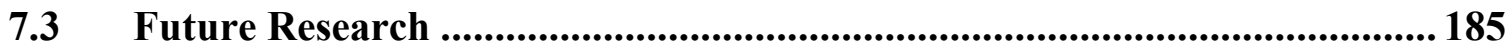

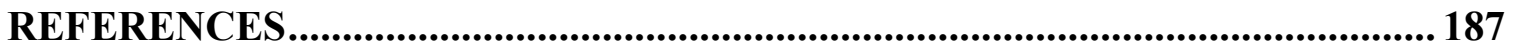

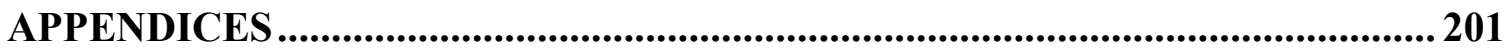

Appendix A: Human Subject Agreement..................................................................... 201

Appendix B: Invitation Letter .......................................................................................... 203

Appendix C: Content Validity Instrument ....................................................................... 204

Appendix D: Judgment Quantification Instrument.....................................................2213 


\section{List of Tables}

Table 1: Projections and distribution of the total population by age between 2015 and 2060. (number in thousands) (Source: United States Census Bureau, 2016).

Table 2 : Physician supply and demand projections, $2015-2025 \ldots \ldots \ldots \ldots \ldots \ldots \ldots . . .5$

Table 3 : Four types of health information technology (HIT) ......................... 8

Table 4 : Phases of telemedicine development (Makena and Hayes, 2011[36]) 23

Table 5: The difference between projection and scenario (SOURCE: Liu, Li \&

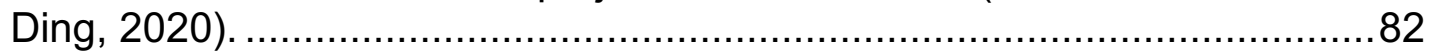

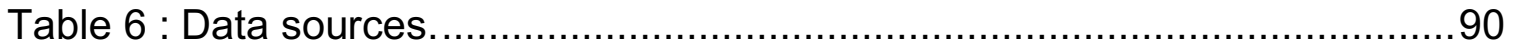

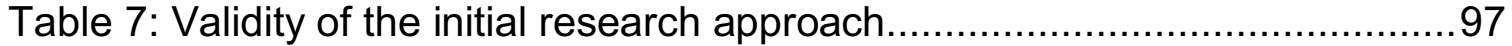

Table 8: Expert panel focus and required expertise....................................121

Table 9 : Total of experts and their background........................................123

Table 10: Focus and number of participants per content validity instrument ....124

Table 11: Summary of responses for content validity instrument 1 ................125

Table 12: Summary of responses for content validity instrument 2 .................125

Table 13: Summary of responses for content validity instrument $3 \ldots \ldots \ldots \ldots \ldots \ldots . .126$

Table 14: Summary of responses for content validity instrument $4 \ldots \ldots \ldots \ldots \ldots \ldots . .126$

Table 15: Summary of responses for content validity instrument $5 \ldots \ldots \ldots \ldots \ldots \ldots . . .126$

Table 16: Summary of responses for content validity instrument $6 \ldots \ldots \ldots \ldots \ldots \ldots . .127$

Table 17: Summary of responses for content validity instrument $7 \ldots \ldots \ldots \ldots \ldots \ldots . . .127$

Table 18: Summary of responses for content validity instrument $8 \ldots \ldots \ldots \ldots \ldots \ldots . . .128$

Table 19: Analysis of expert panel 1 results ..............................................134

Table 20 : Analysis of Subgroup "A" results in expert panel 1 ........................135

Table 21: Analysis of Subgroup "B" results in expert panel 1 ........................136

Table 22: Analysis of expert panel 2 (A) results.........................................139

Table 23 : Analysis of expert panel 2 (B) results ..........................................139

Table 24 : Analysis of expert panel 3 results ............................................. 141

Table 25: Analysis of Subgroup "A" results in expert panel 3 ....................... 142

Table 26 : Analysis of Subgroup "B" results in expert panel $3 \ldots \ldots \ldots \ldots \ldots \ldots \ldots \ldots \ldots . . .143$

Table 27 : Analysis of expert panel 4 results ................................................. 145

Table 28: Analysis of expert panel 5 results ............................................146

Table 29: Analysis of Subgroup "A" results in expert panel 3 .......................148

Table 30 : Analysis of Subgroup "B" results in expert panel $3 \ldots \ldots \ldots \ldots \ldots \ldots \ldots \ldots . . . .148$

Table 31: Analysis of expert panel 6 results ............................................150

Table 32 : Analysis of Subgroup "A" results in expert panel 6 ........................151

Table 33 : Analysis of Subgroup "B" results in expert panel 6 .......................151

Table 34 : Analysis of expert panel 7 results ...............................................153

Table 35 : The relative importance of all sub-criteria with respect to the mission

156

Table 36 : The overall importance of alternative with respect to the mission ....158

Table 37 : Relative importance of subcriteria per subgroups in panel 3........... 159

Table 38 : Ranking of alternative solutions per subgroups in panel 3..............159

Table 39 : Relative importance of sub-criteria per subgroups in panel 5 .........160 
Table 40 : Ranking of alternative solutions per subgroups in panel $5 \ldots \ldots \ldots \ldots . . . .160$

Table 41: Relative importance of subcriteria per subgroups in panel 6............161

Table 42: Rankings of alternative solutions per subgroups in panel 6 .............161

Table 43: Distribution of weights for model perspectives in four scenarios .......162

Table 44 : Description of the four scenarios focus. .......................................163

Table 45 : Scenario 1 overall importance of alternatives ................................164

Table 46 : Scenario 2 overall importance of alternatives ...............................165

Table 47: Scenario 3 overall importance of alternatives .................................167

Table 48 : Scenario 4 overall importance of alternatives ...............................168

Table 49: The global weight for the subcriteria ........................................173

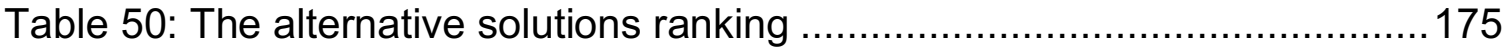




\section{List of Figures}

Figure 1: National health expenditures in billions (green) and the constant (blue) between 1970 - 2018. (Source: http://www.cms.gov) …........................... 3

Figure 2 : Physician supply and demand projections, 2013 - 2025. (Source

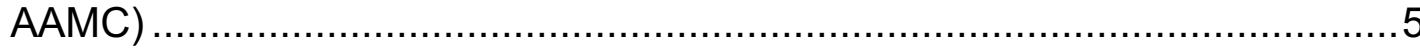

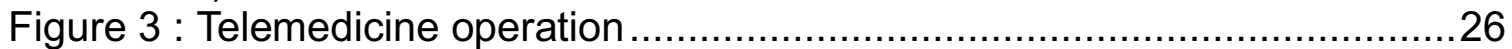

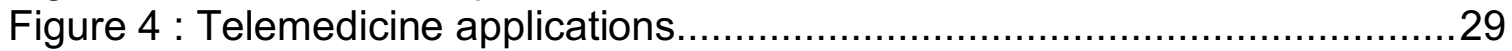

Figure 5 : How Remote Patient Monitoring (RPM) works................................40

Figure 6 : Some Remote Patient Monitoring (RPM) devices. (Source:

http://www.healthcare.philips.com) .................................................4 41

Figure 7 : Telemedince important to healthcare organization. (Source: Foley

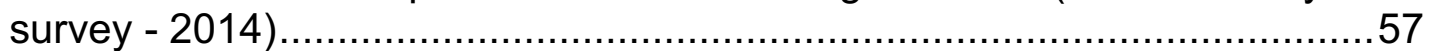

Figure 8 : Top telemedince practices currently available to patients. (Source: Foley survey - 2014) .............................................................. 58

Figure 9: Current status of telemedince program. (Source: Foley survey - 2014)

Figure 10 : Worldwide revenue telehealth devices and services 2013-2018.

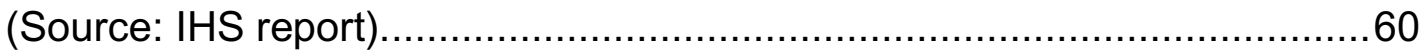

Figure 11 : Forecasted number of telehealth patients worldwide 2013-2018.

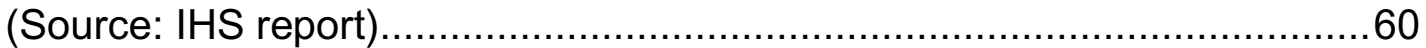

Figure 12 : Literature review, goal and questions. ....................................67

Figure 13 : Five levels of the Hierarchical Decision Model (HDM) ....................75

Figure 14 : The most significant concern regarding reimbursement of telemedince services. (Source: Foley survey - 2014)...........................107

Figure 15 : Theory of Reasoned Action (TRA) ( Aizen and Fishben. 1980) [119].

Figure 16 : Theory of Planned Behavior (TPB) (Ajzen, I. 1991) [126].............. 116

Figure 17 : Final version of Technology Acceptance Model (TAM) [127] .........117

Figure 18 : Initial research model.........................................................122

Figure 19 : The finalized assessment model............................................129

Figure 20 : Relative importance of perceived ease of use and perceived

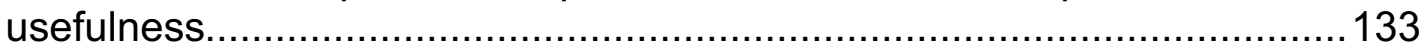

Figure 21 : Subgroups in expert panel 1 using dendrogram .........................135

Figure 22 : Relative importance of criteria with respect to the mission (A)........137

Figure 23 : Relative importance of criteria with respect to the mission (B)........138

Figure 24 : Relative importance of sub-criteria under financial barriers ............140

Figure 25 : Subgroups in expert panel 3 using dendrogram ..........................142

Figure 26 : Relative importance of sub-criteria under technical barriers ........... 144

Figure 27 : Relative importance of subcriteria under logistical barriers.............145

Figure 28 : Subgroups in expert panel 5 using dendrogram ......................... 147

Figure 29 : Relative importance of sub-criteria under cultural barriers.............. 149

Figure 30 : Subgroups in expert panel 6 using dendrogram .........................150

Figure 31 : Relative weights of alternatives with respect to the mission ...........152

xiii 
Figure 32 : Relative importance of sub-criteria with respect to the mission ......155 Figure 33 : Overall importance of alternative solutions with respect to the mission 157

Figure 34 : The global contribution of alternatives to the mission in scenario 1163 Figure 35 : The global contribution of alternatives to the mission in scenario 2165 Figure 36 : The global contribution of alternatives to the mission in scenario 3166 Figure 37: The global contribution of alternatives to the mission in scenario 4167 Figure 38: Grouping the alternative solutions ……..................................176

Figure 39: The final model weight ........................................................ 180 


\section{CHAPTER 1: INTRODUCTION}

Today, there are rapid and dynamic changes in all business sectors that increase critical and valuable technology management to be competitive for any organization's survival. Some factors should be given attention by any organization to improve its performance to be successful, such as responsiveness to new technological opportunities, and the ability to identify, evaluate, and adopt the latest technologies. [1]. The healthcare sector is one of the most important areas for any country for two reasons: first, it is related to people's health and lives, and second, it has high costs for both developing and industrial countries.

Healthcare is a critical issue in the United States and the entire world. The United States spends about $18 \%$ of its Gross Domestic Product (GDP) on healthcare, which is more than what any other developed country currently spends. The United States has led the world in both the level and growth rate of spending on healthcare [2]. The United States government suffers from rising healthcare costs, and in many cases, these increases are passed on to American families by paying higher premiums or out-of-pocket spending [3]. Moreover, a growing population and a shortage of physicians are complicating the healthcare issue with more challenges. Telemedicine was introduced as an implementation tool to improve and support healthcare services and will offer considerable changes in healthcare organizations [4]. The impact of telemedicine will reach all healthcare organizations, from physicians to nursing, and throughout all levels. 
A study done by the Agency for Healthcare Research and Quality (AHRQ) indicates that telemedicine is a small but growing movement and concludes, "active programs demonstrate that the technology can work, and their growing number indicates that telemedicine can be used beneficially from clinical and economic standpoints" [5]. The same study identified 455 telemedicine programs of which $80 \%$ are in the United States. The top three common telemedicine activities for these programs were consultations or second opinions (290 programs), diagnostic test interpretations (169 programs), and chronic disease management (130 programs) [5].

The objective of this research is to evaluate the solutions which will remove barriers so that HIT can diffuse and adopted.

\subsection{The Increase of Health Expenditure}

According to statistics compiled by researchers at the Centers for Medicare and Medicaid Services (CMS), the total United States healthcare spending in 2013 reached $\$ 2.9$ trillion or $\$ 9,255$ per person [6]. The GDP grew similarly in 2010 to 2013, and health spending as a share of the GDP has remained stable from 2009 through 2013 , at $17.4 \%$ as shown in figure 1 (blue curve). The total healthcare spending in the United States has increased from $\$ 75$ billion in 1970 to $\$ 3.6$ trillion in 2018 as shown in figure 1 (green curve).

Nevertheless, healthcare expenditures will keep increasing according to the recent study by the Center for Medicare and Medicaid Services (CMS) and it will 
reach $\$ 4.8$ billion by the end of 2021 , about one-fifth or $20 \%$ of the United States GDP [6].

Total national health expenditures, US \$ Billions, 1970-2018

- Total National Health Expenditures - Constant 2018 Dollars

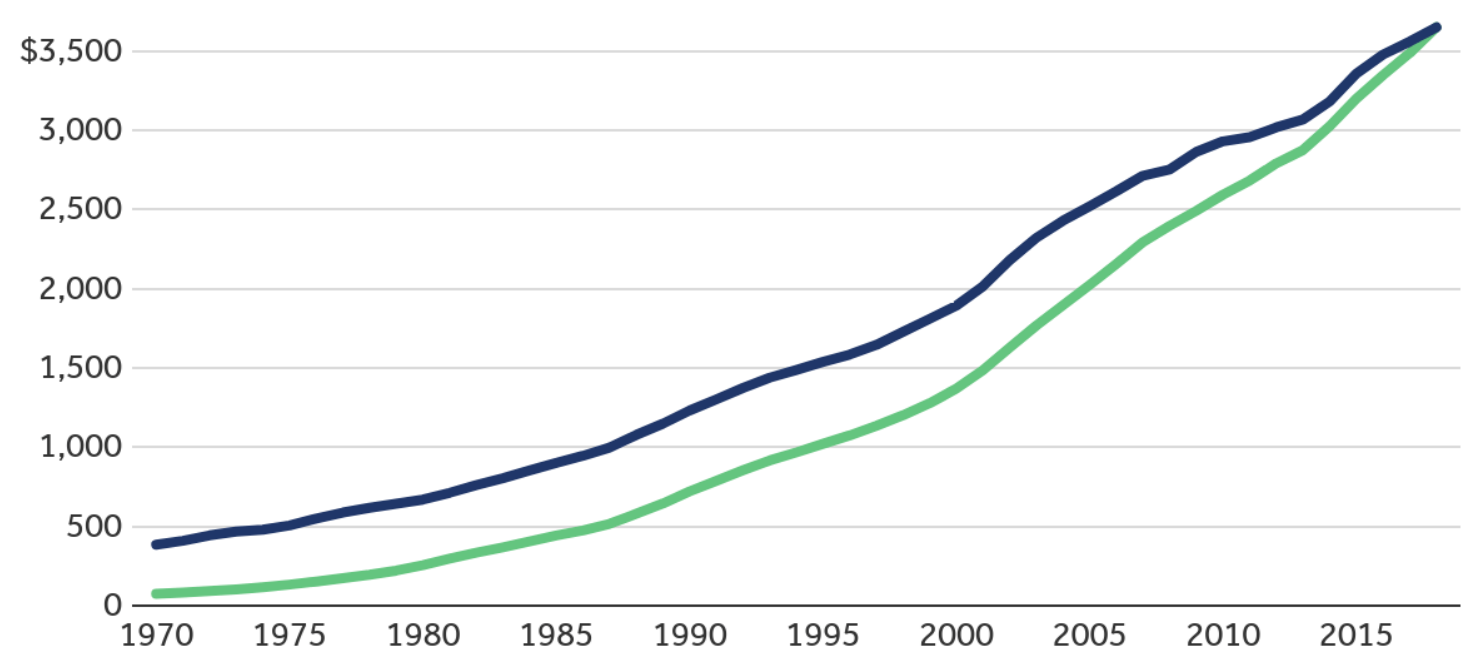

Figure 1: National health expenditures in billions (green) and the constant (blue) between 1970 2018. (Source: http://www.cms.gov)

\subsection{A Growing United States Population}

According to the United States Census Bureau, between 2013 and 2023 the country's population will increase by 9.5 percent [7] . Also, in the same study the Census Bureau projects that the population aged 65 and older will increase by nearly 45 percent [7]. As the elder population increases, the need for healthcare services and products associated with the amplified prevalence of complex medical conditions and chronic diseases will continue to rise.

According to another study, by 2050 the total elder population (65 years and over) will reach about 88 million or more than double the 40.5 million elderly people 
in 2010 [8]. Also, by 2030 the total elder population (65 years and over) will reach above $20 \%$ of the total population of the country, projected to reach more than 439 million as shown in Table $1[7,9]$. Moreover, by 2023 about an additional 28 million people will have health insurance, according to an estimate done by the Congressional Budget Office [8].

\begin{tabular}{|c|c|c|c|c|c|c|c|c|}
\hline \multirow{2}{*}{ Age Group } & \multicolumn{6}{|c|}{ Population } & \multicolumn{2}{|c|}{ Change, 2015 to 2060} \\
\hline & 2015 & 2020 & 2030 & 2040 & 2050 & 2060 & Number & Percent \\
\hline \multicolumn{9}{|l|}{ NUMBER } \\
\hline Total & 323,127 & 334,503 & 359,402 & 380,219 & 398,328 & 416,795 & 93,668 & $29 \%$ \\
\hline Under 18 & 78,181 & 74,128 & 76,273 & 78,185 & 79,888 & 82,309 & 4,128 & $5 \%$ \\
\hline 18 to 44 & 114,826 & 120,073 & 126,588 & 128,669 & 132,371 & 136,310 & 21,484 & $19 \%$ \\
\hline 45 to 64 & 82,574 & 83,861 & 82,434 & 91,021 & 98,074 & 100,013 & 17,439 & $21 \%$ \\
\hline 65 and older & 47,546 & 56,441 & 74,107 & 82,344 & 87,996 & 98,164 & 50,618 & $106 \%$ \\
\hline \multicolumn{9}{|l|}{ PERCENT \% } \\
\hline Total & $100 \%$ & $100 \%$ & $100 \%$ & $100 \%$ & $100 \%$ & $100 \%$ & & \\
\hline Under 18 & $24.2 \%$ & $22.2 \%$ & $21.2 \%$ & $20.6 \%$ & $20.1 \%$ & $19.7 \%$ & & \\
\hline 18 to 44 & $35.5 \%$ & $35.9 \%$ & $35.2 \%$ & $33.8 \%$ & $33.2 \%$ & $32.7 \%$ & & \\
\hline 45 to 64 & $25.6 \%$ & $25.1 \%$ & $22.9 \%$ & $23.9 \%$ & $24.6 \%$ & $24.0 \%$ & & \\
\hline 65 and older & $14.7 \%$ & $16.9 \%$ & $20.6 \%$ & $21.7 \%$ & $22.1 \%$ & $23.6 \%$ & & \\
\hline
\end{tabular}

Table 1: Projections and distribution of the total population by age between 2015 and 2060 . (number in thousands) (Source: United States Census Bureau, 2016).

\subsection{A Projected Physician Shortage}

According to a new study, the United States will face a growing shortage of physicians and will need more doctors by 2025 , a need driven by an aging population and increased access to health insurance. Overall, the researchers projected that the demand for primary care services or family doctors will grow by approximately 17 percent between 2013 and $2025[8,10]$.

The American Association of Medical College Center (AAMC) for workforce studies reports that the demand for physician services is increasing rapidly as the nation's population continues to grow, live longer, and have more chronic illnesses 
that require increased medical attention. Also, about one in three physicians in the United States is 55 years or older and nearing retirement age [11, 12]. The AAMC projected the shortage of physicians based on the supply and demand pattern which will reach over 110,000 by 2025 as shown in table 2 and Figure 2 [13].

\begin{tabular}{|c|c|c|}
\hline Year & $7^{\text {th }}$ Percentile & $2^{\text {th }}$ Percentile \\
\hline 2015 & 30,800 & 21,800 \\
\hline 2016 & 39,300 & 26,900 \\
\hline 2017 & 46,100 & 29,600 \\
\hline 2018 & 52,700 & 31,700 \\
\hline 2019 & 58,500 & 33,400 \\
\hline 2020 & 65,500 & 35,100 \\
\hline 2021 & 71,500 & 37,200 \\
\hline 2022 & 79,200 & 39,600 \\
\hline 2023 & 83,200 & 41,300 \\
\hline 2024 & 86,700 & 43,900 \\
\hline 2025 & 94,700 & 61,700 \\
\hline
\end{tabular}

Table 2 : Physician supply and demand projections, 2015 - 2025.

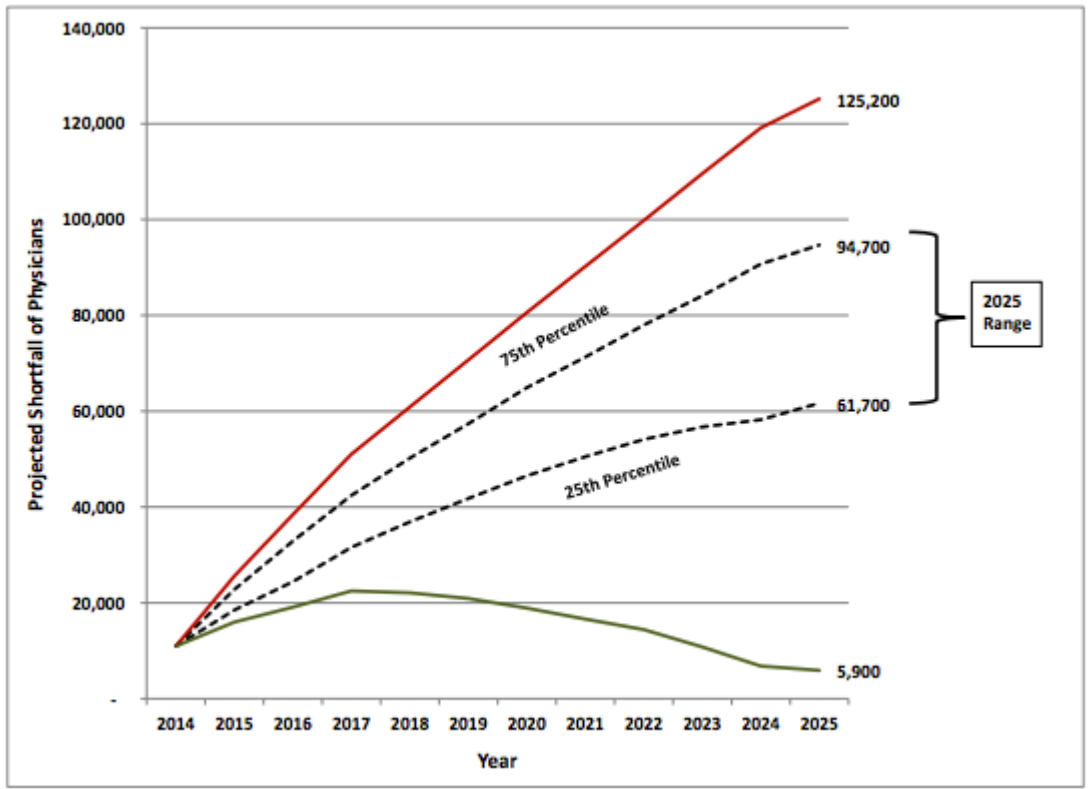

Figure 2 : Physician supply and demand projections, 2013 - 2025. (Source AAMC) 


\section{CHAPTER 2: LITERATURE REVIEW}

The literature highlights researchers from different fields and with several interests in recognizing the important role of Health information technology (HIT) in the healthcare industry. There are many researchers conducting studies in order to recognize the important of using of HIT.

\subsection{Health Information Technology (HIT).}

Health information technology offers a wide range of new technologies intended to deal with healthcare information. The most essential kind of health information technology is a system that automatically captures, saves, and stores health information about patients electronically. When appropriately put in place, it can be used in healthcare facilities to save papers and improve accuracy of a patient's information. There are several types of HIT as shown in table 3.

The electronic health record (EHR) is the major component of HIT infrastructure [16]. It keeps a person's digital health record and is shared among different facilities and branches. People give information collected electronically different names such as the electronic health record (EHR) and others call it electronic medical record (EMR). Some health experts differentiate the two but, in most cases, they are used interchangeably. Much research is underway to find distinct difference between the two names.

This system has been highly implemented lately with the introduction of the Health Information Technology Act in 2009, which included a meaningful use program in Electronic Health Record (EHR). Hospitals, doctors, and healthcare 
professionals who use EHR systems must comply with the meaningful use criteria. These activities are usually monitored by Centers for Medicare and Medicaid services as well as the health information technology national coordinator.

Another type of HIT is referred to as a personal health record (PHR). It maintains individual health records that are not shared with either facilities or agencies. Also, we have the Regional Health Information Organization (RHIO) which is the overall overseer of communication among other systems that unites them geographically.

The other type of health information system is the picture archiving and communication systems (PACS), which help physicians in storage and maintenance of customer's medical images. Before installing PACS in a radiology department, the facility should first plan on how to integrate PACS with other existing health information technology systems.

Vendor neutral archives (VNAs) is the final type of health information technology. It helps physicians in maintaining patients' medical images. VNAs have in some cases been designed to put together and merge imaging data kept in different PACS that share the same multi-factory healthcare system. 


\begin{tabular}{|l|l|}
\hline Types of Health Information Technology (HIT) & \multicolumn{1}{|c|}{ Definition } \\
\hline Electronic Health Record (EHR) & $\begin{array}{l}\text { Is an electronic version of a patient's } \\
\text { medical history that is maintained by the } \\
\text { provider over time, and may include all of } \\
\text { the key administrative clinical data. }\end{array}$ \\
\hline Personal Health Record (PHR) & $\begin{array}{l}\text { Is an electronic application used by } \\
\text { patients to maintain and manage their } \\
\text { health information in a private, secure, and } \\
\text { confidential environment }\end{array}$ \\
\hline $\begin{array}{l}\text { Picture Archiving and Communication Systems } \\
\text { (PACS) }\end{array}$ & $\begin{array}{l}\text { Is a healthcare technology for the short- } \\
\text { and long-term storage, retrieval, } \\
\text { management, distribution and presentation } \\
\text { of medical images. }\end{array}$ \\
\hline Vendor Neutral archives (VANs) & $\begin{array}{l}\text { Is a medical imaging technology used by } \\
\text { healthcare professionals that stores } \\
\text { images in a standard format and interface, } \\
\text { making medical imaging data accessible } \\
\text { through different picture archiving systems. }\end{array}$ \\
\hline
\end{tabular}

Table 3 : Four types of health information technology (HIT).

Updates to health information technology have been done due to increased patient data breaches [17]. For instance, mobile health devices have been introduced as well as updates related to adoption of health information technology systems. For example, EHR has adopted a second stage called compliance apart the first stage of meaningful use program.

Healthcare facilities handle patient's information with care. For instance, Health Insurance Portability and Accountability Act (HIPAA) privacy rule monitor health information technology systems that deal with protected health information $(\mathrm{PHI})$. This rule was created by the United States Department of Health and Human Services to protect the rights of patients by ensuring that their information is confidential by limiting its access to people other than the patient themselves. If 
healthcare facilities do not protect the rights of a patient, they pay fines to the civil rights office.

A lot of people in the United States have elevated hopes for health information technology in the health sector. Hospitals hope to reduce health errors, such as ordering and administering the wrong dose of a medication. There are those who provide hope to access and share patient's information more easily, thereby improving care. The state and private businesses hope to save money by improving competence.

According to RAND Corporation researchers, full completion of health information technology systems could create competence savings as huge as $\$ 77$ billion per year. But for a diversity of reasons, healthcare providers have not fully embarked on this new technology. Some experts look at the costs associated with implementation, resistance to change, and failure to adopt new technologies. Some research suggests that implementing HIT systems may stop patient care at the initial stages.

Even though the United States spends more on healthcare than any other developed country, the rate of infant mortality and its maternal healthcare is not any different from that of the developing countries. Currently roughly $16 \%$ of the United States population lacks health insurance [18]. The American healthcare system currently has not yet achieved universal healthcare.

Furthermore, the United States healthcare facilities are now recruiting foreign nurses [19]. The United States healthcare system is straining to fill current 
nurse vacancies causing an under-supply concern, predicted to be the case for the next twenty years. This has led to outsourcing of nurses which is costlier.

In the United States, the cost of a medical healthcare is very expensive. Most of the people are struggling to get their health insurance premiums. In every ten employees, only $50 \%$ were covered by their employer in 2012 . Those who were covered got a $7.2 \%$ increase in their share of healthcare costs between 2011 and 2012. Research also found out that 49 million Americans did not have health insurance in 2011 [18].

There has been an increasing problem of paying for healthcare. About $26 \%$ of the population reported that they had a problem with paying for their healthcare costs. Moreover, $58 \%$ of those who try paying end up giving up with the payments. The share increases in healthcare costs has also affected the state budgets, putting other activities at a standstill since there are not enough resources to manage them all.

A 2010 report stated that the United States spends nearly $\$ 2.6$ trillion in 2010 and more than $50 \%$ of this spending went to payments of healthcare services in hospitals. The reason why healthcare in United States is very expensive is that most healthcare facilities are owned by the private sector. Even though private healthcare administrative costs receive notable pressure from politicians, they make up the biggest portion in healthcare facilities in the United States. According to the World Health Organization (WHO), the United States spends more on healthcare than any other country. 
According to National Health Expenditure data, there has been a growth in premiums related to costs of medical care from 2000 to 2010 . The trend has been consistent throughout the decade. The healthcare prices in the United States are $60 \%$ higher than that of other Organization of Economic Cooperation and Development nations. Spending on physicians and other specialists in the United States is almost two and half times higher than any other country.

This data also showed that hospital costs increased by $4.6 \%$ in 2011 . These hospital costs are increasing due to consolidation of the hospital industry. The same data showed that mergers and acquisitions increased by $33 \%$ in 2009 and 2010. Apart from acquisitions, hospital costs increase because of hospital market concentrations. The medical technology in the United States has highly improved and has become a notable contributor of higher healthcare spending. Medical technology accounts for $38 \%$ to $65 \%$ of increased costs. The new technology is very effective to patients but has replaced low-cost options with higher-cost services. Unnecessary tests and procedures have also led to increased medical costs in the United States.

Price Water House Coopers shows that $\$ 1.2$ trillion is due to waste. This wasteful spending is contributed to by failure to follow medical prescriptions, drug abuse, and unhealthy living lifestyles which leads to obesity. Increased chronic diseases have also highly contributed to increased healthcare costs. Most of these diseases are preventable and not curable and therefore most people frequently visit the hospital, raising medical costs. These chronic diseases have been predicted to increase by $42 \%$ in 2023 . Also, the increased aging population has 
significantly increased the cost incurred in medical healthcare. The buyers of healthcare include the citizens of the United States. This includes all categories with and without insurance policies. People from other countries highly trust the quality of the United States healthcare and therefore travel all the way to America to be treated.

The United States President Barrack Obama has been keenly looking into the issue of healthcare in his country. The President in collaboration with the Council of Economic Advisors (CEA) has established a new healthcare reform which is expected to positively impact the American economy. The healthcare reforms are stated as: the government will be expected to lower the annual growth rate of healthcare costs by $1.5 \%$ which is expected to increase the gross domestic product (GDP) by over $2 \%$ in 2020 and almost $8 \%$ in 2030 . A family of four will be expected to have an income on $\$ 2600$ or higher by 2030 since with increased healthcare services the income gains are still substantial. With a decrease in healthcare costs it is expected that the federal budget deficit will decrease. The living standard of Americans is expected to improve once healthcare services costs are decreased.

$\checkmark$ Advantages of Using Health Information Technology:

- Increases work efficiency

- Reduces paper waste

- Allows easier access to a patient's medical records

- More complete and current medical history 
$\checkmark$ Disadvantages of Using Health Information Technology:

- Expensive to set up

- May not be compatible everywhere

- May require extra training

- Raises ethical and security concerns

The roles of physicians, medics, nurses, and other healthcare professionals are changing. These providers are more reliant upon the clinical content within the Health Information Technology tools, often referring to "decisions" made by these instruments. Such examples include prescribed diagnostic, differential, and therapeutic treatments, choice of drug, and drug dose calculations [20], although the previous explanations for not considering such HIT tools as medical devices is based upon the intermediation of the service provider between the recommended clinical activity and the actual actions. They are taken on behalf of the patient, and the firm reliance on these very sophisticated HIT tools today puts this premise into question [21].

The most important use for HIT may be to help reduce medical errors. This technology-based policy has been confirmed efficient in dropping the effects of human error in the health sector. A clinical health information system may make a considerable impact on medical quality and safety by integrating relevant, automatic decision making and knowledge gaining tools into the practice of therapeutic providers, thereby reducing errors of oversight that result from gaps in supplier awareness or the breakdown to synthesize and apply that knowledge in clinical performance. These systems, when integrated within a larger health 
information system, may improve medical decision making and appropriate use of diagnostic tests and therapeutic agents [20].

However, most medical organizations and providers have been slow to adopt HIT. Recent surveys of computerized physician order entry (CPOE) use show that only $9.6 \%$ of hospitals have CPOE completely available for use, and only half of these hospitals require use of CPOE [20]. In the ambulatory setting, recent estimates place the use of electronic health records (EHR) at $6 \%$ to $15 \%$ of office-based physicians.

The potential advantages of widespread adoption of HIT in our nation's healthcare system make it vital to examine the scientific evidence that currently supports the relative costs and benefits of HIT, and the barriers to implementing various types of HIT systems across the spectrum of healthcare environments.

Although the adjudicators have yet to verify whether HIT will bring extensive positive improvements in effectiveness or less expenditure and high quality of healthcare, there are several operational examples of health information technologies. For instance, The Department of Veterans Affairs has significantly enhanced the quality of healthcare at its amenities that are fitted with new technology. Furthermore, vaccination information systems have shown that they can really be lucrative in managing public health data.

\subsection{Cultural Change in Healthcare Industry}

Since the technology introduction in several organizational sectors including healthcare, people have seen several changes compared to how things were before. First, in healthcare, technology is playing a large role in almost all the 
processes and services that hospitals are offering. All healthcare institutions are supporting and depending on the techniques while carrying out their duties and they are ending up giving the best services to their customers [22]. Second, the cost of medication is reducing dependency on the hospitals that are using technology services. Hospitals that are using technology are lucky and are getting more profit compared to those that are not using the technology. Before the technology changes in healthcare, delivering services was sometimes difficult for professionals since they did not have immediately informed care.

Everyone globally wants a better medication and those hospitals that are giving best services are getting more patients compared to others [23]. In the past, before technology usage in the hospitals, patients were having difficulties in accessing medical information. Some individuals were dying due to lack of enough information on particular illnesses. Those who were afraid to go and see the doctors were also dying, and sometimes people were finding it difficult to manage their health conditions. In the hospitals, patients who needed emergency care were sometimes dying for lack of immediate care. People are seeing a significant change and improvement on how hospitals are accessing medical information, and this is due to technology. With technology, a patient can communicate with a physician concerning their illness even when the doctor is not around. The doctors can explain and give instructions or prescriptions to their patients who are at home [24]. Patient safety tools, such as alarms, are helping those who need an immediate attendant. 
Technology is improving record keeping in the health sector by using the electronic health record. Most doctors are using computers to store data records of their patients. The machines that they are using have various systems. For example, one system will record pharmacy information, and another will order while, yet another system completes the documentation. Doctors are finding it very comfortable to follow the records stored in computers rather than hard document files. Before the introduction of technology in most hospitals, it was tough to trace the records of a patient. Doctors were misplacing files containing records; they were ending up carrying out tests on the patient hence providing late treatment. Technology is now helping them with immediate access to the files of various patients, allowing them to attend to patient care immediately. After carrying out tests, they are easily fitting the results and matching them with the patients' names in their computers, allowing for ease and improved time in following the records.

Carrying out tests in the laboratory is changing and improving with time in various hospitals. Several machines that are using technology are helping in doing this. Some time back, it was tough to carry out laboratory tests for patients within a day since doctors were manually doing the services. Doctors were taking time during the testing process because they wanted to be accurate. Right now, there is a computerized automation in most hospital laboratories that is helping during the specimen tests, hence improving the performance. Almost all the laboratory system tools are using technology, and this is increasing accuracy, giving assistance to doctors in knowing the right treatment. Human errors no longer exist 
in most laboratories because of the machines, and the system is quick to return test results.

Hospitals that are using the tracking tools are always satisfied. Before the introduction of this technology, doctors used to make errors while carrying out surgeries for patients, hence leading to death. Doctors were not accepting any correction from nurses-a situation that is improving due to the tracking tools. Most hospitals are installing these devices in places where doctors or patients cannot see them and only administrators are accessing this device. The doctor cannot give poor medication when they know that the tracker is in the room and this is making them perform their best, hence improving the healthcare of a patient. The tracking tool again is enhancing the flow of the patients that the hospitals are admitting. The tools are giving information about the patients.

\subsection{Telemedicine}

Telemedicine offers great promise in addressing some of the challenges raised by the trends introduced early and is increasingly being included in discussions of the medicine's future. Telemedicine offers the capabilities to deliver healthcare across distances at reduced costs while maintaining or even increasing the quality of treatment and services. The term 'telemedicine' is a general word that encompasses various definitions. It can be defined as the use of medical data conveyed from one site to another through electronic infrastructures to improve a patient's medical status. Telemedicine encompasses an evolving assortment of applications and amenities that are dependent on the use of two-way video, electronic mail, smart phones, wireless tools and many other media of 
telecommunications technology. It can also be defined as the practical implementation of telecommunication and wireless technology to provide medical care at a distance.

\subsubsection{Definition of Telemedicine}

Telemedicine refers generally to the use of advanced information and communication technologies (ICTs) in delivering and supporting healthcare services. Over the past few years, it has been defined and described in several ways. However, the term telemedicine has a much broader meaning for many researchers. It encompasses the whole range of medical applications and services including prevention of disease, diagnosis and treatment, continuing education of healthcare providers and consumers, and allowing patient access to expert advice and information no matter where the patient or relevant information is located [25].

The definition of telemedicine is different from one organization to another, and from industrial to academia fields. Because of this broad scope, there are many definitions and categories of telemedicine depending on the background and perspective. The American Telemedicine Association defined telemedicine as "the use of medical information exchanged from one site to another via electronic communications to improve patients' health status" [26].

The World Health Organization (WHO) defined telemedicine as "The delivery of healthcare services, where distance is a critical factor, by all healthcare professionals using information and communication technologies for the exchange of valid information for diagnosis, treatment and prevention of disease and injuries, 
research and evaluation, and for the continuing education of healthcare providers, all in the interests of advancing the health of individuals and their communities." [27]. The European Commission (EC) defined telemedicine more specifically as "Telemedicine is the rapid access to shared and remote medical expertise by means of telecommunications and information technologies, no matter where the patient or the relevant information is located." [28].

\subsubsection{History of Telemedicine}

For many centuries, healthcare services have been provided to patients with healers using the available communication services of their era. Ancient Egypt established a three-step system that exists to this day. The first step in this system is to listen to the patient, before any examination. Then, a diagnosis is given after observation and examination. The last step is treatment [29].

From this system we can figure out how significant observation and examination is before any treatment is delivered to the patient. Distance healthcare existed for more than 1500 years, and there were many elements that encourage this practice such as limited transportations system, slow travel, and healing at home.

For example, mail was one of the first communication services used, then the telegraph was used in the American Civil war [30]. Healthcare utilized radio after its innovation for long distance international communication [31]. Using the telecommunication network in order to get medical examinations and evaluations is not new. The discovery of radio brought opportunities for distance diagnostics. 
During the early 1900s the people living in remote Australian areas communicated with the Royal Flying Doctor Service of Australia by using two-way radios powered by a dynamo driven by a set of bicycle pedals [29].

In 1903 the history recorded the first experience of using telecardiology by sending electrocardiograph (ECG) from a hospital to a laboratory by Einthoven. Electrocardiograph (ECG) can be transferred using two ways, either by telephone or wirelessly. During the first quarter of the 20th century a huge innovation of broadcasting brought more attention in how to gain advantages from radio communications in medicine [29].

During the mid-1920s both Alfred Traeger and John Flynn started their experiments with radio to connect the distant stations to the centralized medical base in order to replace the telegraph that was very complicated compared to radio. The invention of the telephone brought many ideas in the use of experts to examine and evaluate the transmission of heart and lung sounds, but unfortunately all failed due to poor transmission systems [29].

In 1924, science recorded one of the first conceptions of telediagnostic and televisit as they are used today, which are distance diagnostic examinations of children with direct imaging. During the 1920 s telemedicine was very useful, where physicians at the coast station assisted ships with medical emergencies in the middle of the ocean by using radios. In April 1924, the "Radio News" magazine included an article about telecare and put it on its cover page [29]. 
In 1950 the history recorded the first broadcasts of radiological images between hospitals based on radio communications. The invention of television brought a great impact on development of telemedicine. In 1955, the Nebraska Psychiatric Institute used the first interactive video communication in healthcare by getting advantages from two-way interactive television system for telepsychiatry purposes [32].

In 1967 the General Hospital in Massachusetts provided health services to airport employees and passengers. They used a two-way audiovisual microwave circuit (located 2.7 miles away) thus providing medical care to the patients at Logan International Airport Medical Station [33]. In 1971 the Nebraska Medical Center was linked with the Omaha Veterans Administration Hospital and VA facilities in two other towns [29].

Telemedicine officially began in the early 1960s, in the United States of America, when the National Aeronautics and Space Administration (NASA) first sent men into space [34]. The medical dimensions of the space pilots were telemetered from both the spaceship and the space uniforms throughout NASA space flights.

In the 1960s, because of the need to monitor astronauts' vital signs, the NASA played a crucial role in the early development of telemedicine. The Space Technology Applied to Rural Papago Advanced Healthcare (STARPAHC) program was established by means of satellite-based communication and space technology so as to bring medical care to the astronauts and residents of Papago, Arizona 
[35]. These previous efforts were heightened by the development of cable technology that nurtured the development of telemedicine.

In the 1970's Europe started the first telemedicine experience in Scotland by providing remote health attention to workers in the North Sea at the oil platforms. In other European courtiers, such as Norway, the national programs started to provide some help to remote rural centers. Also during the 1970 s, satellite telemedicine began Via ATS- 6 satellites, and the paramedics in isolated Alaskan and Canadian villages were connected with hospitals in distant towns [29]. Table 4 summarizes the phases of telemedicine developments as shown below: 


\begin{tabular}{|c|c|c|}
\hline Time Scale & $\begin{array}{c}\text { Technology } \\
\text { used }\end{array}$ & Examples / Description \\
\hline Mid 19th century & Postal & $\begin{array}{c}\text { Prescriptions and diagnosis exchanged } \\
\text { between patient and physician by posts. }\end{array}$ \\
\hline 1835 & Telegraphy & $\begin{array}{c}\text { Used during American Civil War to send } \\
\text { casualty lists and order supplies }\end{array}$ \\
\hline 1906 & Telephone & Electrocardiograms sent using telephone \\
networks
\end{tabular}

Table 4 : Phases of telemedicine development (Makena and Hayes, 2011[36])

\subsubsection{Types of Telemedicine}

The definition of telemedicine is different from one organization to another and from industrial outlook to academia viewpoint, and the categories of telemedicine are different in various research depending on the background and perspective. There are various types of telemedicine services practiced currently which include, but are not limited to: 
- Emergency Telemedicine

- Tele-Nursing

- Tele rehabilitation

- Tele-audiology

- Tele-Pharmacy

- General Healthcare Delivery

- Tele neuropsychology

- Tele trauma care

- Tele ophthalmology

- Tele-Cardiology

- Remote Surgery

- Tele psychiatry

- Telepath ology

- Tele-Radiology

- Tele dermatology

- Tele Dentistry

Consistent with the World Health Organization (WHO), telemedicine can be classified based on the time of the information transmitted and the interaction between the patient and the health professional in two basic types, store-andforward (asynchronous) and real time (synchronous) [27], [30]. Another classification by the American Telemedicine Association (ATA) classified telemedicine services into five main categories: specialist referral services, patient consultations, remote patient monitoring, medical education, and consumer medical and health information [26]. Another classification done by Takahashi divided Telemedicine into three main categories: direct patient care, teleconsultation, and distant learning [37]. 
After reviewing much literature in categorizing and classifying telemedicine, we could group all of these categories and the classifications into two main categories according to modes of operation and telemedicine applications.

\subsubsection{Modes of Operation}

\subsubsection{Store and forward (asynchronous communication)}

Medical data such as images or videos can be saved and then transmitted to a physician or medical specialist for assessment. Unlike traditional in-person patient meetings and actual physical examination, this method of telemedicine does not require the presence of doctor and patient at the same time. Pathology, dermatology and radiology are common specialties that use this process.

\subsubsection{Real time (synchronous communication)}

This division of telemedicine services involves real-time interactions between a patient and healthcare provider through two-way television or phone conversations. Many traditional physical examination activities that are performed in face-to-face visits can be performed in this way. This approach requires advanced equipment and medical devices to send and receive real-time medical data and employs video conferencing.

\subsubsection{Remote monitoring (self-monitoring/testing)}

This method enables the clinician to monitor and measure patient health data and information remotely by using various devices. It has been used together with advanced communication equipment for managing chronic diseases such as 
Congestive Heart Failure (CHF), Chronic Obstructive Pulmonary Disease (COPD), diabetes, and asthma to achieve earlier and proactive interventions in these diseases. Sensors such as Blood Pressure (BP), weight, pulse oximetry, glucose and electrocardiograph (ECG) can be utilized for remote monitoring of patients. The three modes of operation are shown below in Figure 3:

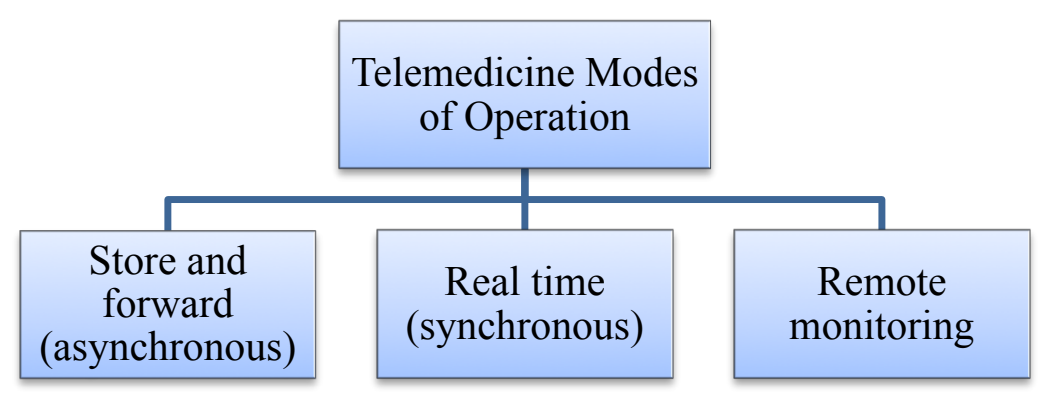

Figure 3 : Telemedicine operation

\subsubsection{Delivery Mediums}

There are various mediums that are utilized in the provision of telemedicine services. They include networked programs which are responsible for connecting tertiary healthcare facilities with remote clinics and communal health hubs in the countryside or residential areas. The links may exploit dedicated high-speed conduits or the Internet for cable links amongst sites. The ATA approximates the sum of current telemedicine systems in the United States at roughly 200 providing coverage to over 3,000 locations [34].

Point-to-point networks using secluded high-speed systems are utilized by infirmaries and clinics that convey services openly or subcontract department services to principal medical service benefactors. Such subcontracted facilities 
include radiology, stroke evaluation, mental health and intensive care amenities. Intensive care center links are utilized for cardiac, pulmonic or fetal specialist care, home care and associated facilities that offer care to patients in the home. Frequently, standard landline or wireless links are used to communicate openly amid the patient and the midpoint although some systems may use the Internet.

Web-based e-health patient facility sites offer direct customer outreach and services over the Internet. Under telemedicine, these comprise those sites that provide direct patient care.

\subsubsection{Telemedicine applications}

\subsubsection{Teleconsultation}

Teleconsultation uses Information and Communications Technologies (ICT) to obtain the opinion of a healthcare provider who is distant from the patient and/or their primary healthcare provider, regarding the diagnosis and treatment of the patient. This type of patient/doctor communication can take place in real time (live consultation) via a videoconferencing link. Data consultation or store-and-forward process is the other form of teleconsultation, which is affected by acquiring the patient's medical information and then sending it to the consultant to give his/her opinion at some time later.

\subsubsection{Telemonitoring (biotelemetry)}

Telemonitoring is the use of audio, video and other telecommunication and information technology to monitor the status of a patient at a distance [38]. It has been used to transmit the physiological and biological data of patients from one 
location, such as the home, office and primary clinic to another for data analysis and decision making. Recently, this method has spread across the world as it has proven to be an effective tool in follow-up treatment and chronic disease management, especially in communities with rising elderly populations. It has also been adopted as a response to the rising needs for home care [38].

\subsubsection{Telesurgery (remote surgery)}

Telesurgery involves the manipulation of the surgical instruments by a surgeon with the assistance of advanced medical devices and telecommunication technology at a remote site from the patient [39]. A robotic system controlled by a remote surgeon directly implements all surgical tasks in real time through an interface and is as precise as traditional surgery.

\subsubsection{Teleeducation (telelearning)}

Teleeducation has been used for many years to deliver continuing education programs to rural healthcare professionals or those with poor access such as in developing countries [40]. Teleeducation has three basic modes that are video, audio and computer. Distance learning can be used in either synchronous or asynchronous modes. Video conferencing and interactive television are examples of synchronous video learning offering live visual and verbal interaction between instructors and learners. The transmission of the spoken word (voice) can be considered as synchronous audio learning [40]. Recently, computer aided learning has become common in distance learning 
manifested through many forms such as the Internet and email. The four telemedicine applications are summarized in Figure 4 below:

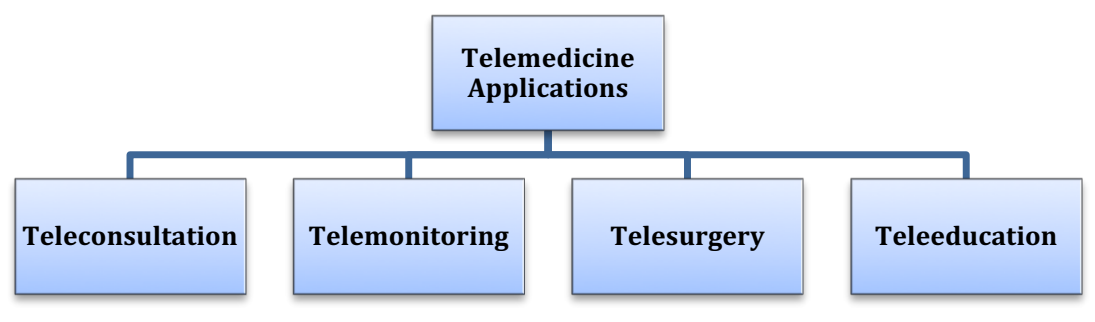

Figure 4 : Telemedicine applications

\subsection{Teleconsultation}

Teleconsultation is an advanced telemedicine application that has spread considerably, especially in developed countries in recent years. Teleconsultation has been defined in many ways. Essentially, consultation means seeking advice or information from somebody. Thus, teleconsultation means seeking medical advice or information from a person at a distance $[25,129]$. This process may take place between healthcare professionals or between patient and physician [130]. Teleconsultation is also defined according to the applied mechanism between health service providers and consumers as "the consultation of one (or more) distant healthcare professional(s) by a locally present healthcare professional about a patient's case, diagnosis and treatment using telecommunication and information technology to bridge the spatial distance between two (or more) participants" [131]. Others have defined the teleconsultation concept in terms of its main purpose of providing medical proficiency or treatment to places where there 
are shortages in expertise by leveraging electronic communications [132, 133]. The clinical aims of teleconsultation are as follows [134]:

- To help make a diagnosis on the basis of clinical history, clinical examination, and investigation.

- To ask the best way to treat a patient and what that treatment should consist of.

- To obtain advice about technical aspects of care or treatment.

- To ask for corroborative opinion in relation to a recommended action for a patient.

- To obtain advice if a patient needs to transfer elsewhere for treatment and how this transfer can be done safely.

\subsubsection{Types of Teleconsultation}

Teleconsultation can be distinguished into two main categories: synchronous (real-time consultation) and asynchronous (store and forward consultation).

\subsubsection{Synchronous (real-time consultation)}

Synchronous consultation involves real time face-to-face contact (image and voice) via video-conferencing equipment such as television, digital camera, webcam or videophone simultaneously connecting medical experts and clients, who are separated geographically [135]. It simulates the traditional physical faceto-face consultation where parties can interact regarding their concerns without any delay in time or data transmission. There is evidence that on-line-consultation has proved effective in treating chronic and severe disease. Compared with asynchronous teleconsultation, this method is expensive, as it requires more 
advanced equipment and medical devices to send and receive real time medical images and data connecting via video link $[135,136]$.

\subsubsection{Asynchronous (store and forward consultation)}

In this approach all the clinical information of the patient (medical record, video and audio recordings) and medical images are captured, processed and analyzed and sent to at least one consultant in other locations for a medical opinion. This process does not require the patient and consultant to be present at the same time or face-to-face. Subsequently, the decision is transmitted by the consultant. Recently asynchronous consultation has been divided into message, storage and discussion systems [135]. The message system utilizes email to enable doctors to send questions directly to other specialists and receive replies. Storage systems include Picture Archive Communication Systems (PACS), which are used in teleradiology. Discussion systems implement the functionality of a typical web based bulletin board system (including messaging, discussions, image storage, etc.) and typically are open to all participants $[137,138]$.

Telephone is the early form of teleconsultation and is still popular in medical care. It is the cheapest of the teleconsultation methods and can be utilized within communities that are less advanced in telecommunication and information technology or are not able to afford the cost of real time interaction.

Teleconsultation has proven its value in healthcare delivery systems and is being applied across many medical practices. Healthcare professionals and patients both benefit from teleconsultation because it is more efficient with their time. Substituting conventional face-to-face contact with video consultation 
becomes irresistible, particularly over large geographies, with hard to access communities or care groups, where clinical expertise is scarce. The circumstances where access to high-speed broadband is becoming available in domestic and communal environments should make this technology widespread over the next few years.

\subsubsection{Advantages of Teleconsultation}

Teleconsultation is, at its core, a way to deliver two important changes to healthcare removes distance instantly and increased choices as to how services are accessed [139].

\subsubsection{Removes distance instantly}

Healthcare professionals and patients are brought together through teleconsultations without having to be in the same location, thus avoiding unnecessary travel and reduce cost [140]. It allows delivering greater levels of productivity and flexibility to healthcare providers. A patient and nurse can attend a hospital outpatient consultation "virtually" from a General Practitioner (GP) surgery through this technology. Teleconsultations can provide great benefit especially where expertise is limited and an instantaneous informed input from that expert can materially affect the treatment plan and thus the outcome for the patient [141].

\subsubsection{Increased choices as to how services are accessed}

Healthcare providers and patients will have a wider menu of effective and conveniences with options to work [142]. Teleconsultation can be the best mode 
for change from one physical channel for accessing services to a choice of channels of coordination between healthcare professionals, their patients and care teams.

\subsubsection{Other advantages}

- Improve quality treatment utilizing medical expertise hence enriching of healthcare [131].

- Reduces diagnostic errors.

- Equality of access to specialists.

- Permits easier follow-up treatment to manage chronic disease.

- Enhances patient self-care.

- Improves patient confidence in diagnosis and treatment.

- Reduces cost for patient by avoiding unnecessary travel and for health system by eliminating waste of resources.

- Increases access to care and reduced burden on hospitals.

- Knowledge transfer.

- Increases medical education.

\subsubsection{Teleconsultation for older patients}

The increasing number of the elderly leads to an uptrend in chronic disease and multi-morbidity, therefore we are focusing on this research only in older patients. Monitoring will become a central part of the treatment concept for many of the patients especially in many rural and remote regions. The spatial distribution of healthcare providers is already sparse, and with a decreasing medical care population, healthcare close to patients' residence will be difficult. Increasing distances will restrict regular visits to practices and clinics for older patients with limited mobility [143]. Telemedicine has the potential to improve their access to 
healthcare. Telemedicine has been used successfully with all age groups. Teleconsultation programs can serve an important function in home healthcare to support older adults in their own homes and communities [144]. Telemedicine and teleconsultation especially is helpful in managing the chronic conditions of those aged 65 and older in the face of all-time-high levels of cardiovascular diseases, diabetes, cancer and obesity [145].

Most patients with Alzheimer's or dementia are the elder people in society and technology can help them recover their memories with the help of tracking devices. Due to memory damage, these patients are at high risk of falling anytime and sometimes this is the leading cause to their deaths. To help these people out, most doctors are fixing the RPM tracker or sensor to the devices of these seniors like the walkers and canes. The tracking device is using the remote control to detect angular velocity changes and steps and gives notification to the caregivers. In the traditional models these services were not available, and older people were falling and dying because in most cases, no one attended to them. Doctors are now helping such individuals with the use of life alerts that are informing them when elderly individuals fall and cannot get up.

Those who have diabetes are monitoring their blood pressure using the continuous glucose-monitoring device that is informing the caregivers in case the blood pressure rises. In any event of an increase in blood glucose or pressure, the device is reading and immediately alerting the patient and the medication providers that are intervening directly. The device is continuously doing the measurement, and most people prefer checking the status every morning when they wake up. 
They are managing diabetes daily using the RPM technology instead of going for the three months' checkup, and this is helping a lot of people because it is reducing the cost of visiting the hospital.

Most individuals living in the United States are dying due to the congestive heart failure. Their cardiac tissues are becoming ischemic and blocked due to the coronary vessels. Once the tissue becomes ischemic, the core machinery functionality is reduced disrupting the normal process of electrophysiological hence leading to congestive heart failure. For the death rate of these people to be reduced, there should be an introduction of machines that can monitoring their heart disease daily. Hospitals that are dealing with heart disease are using several RPM tools like telemedicine, congestive heart failure patients monitoring devices, and wireless health and health monitoring devices that are all improving life quality [146]. The RPM technology is improving the relationship between heart failure patients, with doctors allowing them to be free with each other. The uses of devices are shortening the duration that a patient should stay in the hospital because they are acting quickly towards patient needs, hence the doctor is able to attends to them immediately.

\subsubsection{Teleconsultation Technology}

With the proliferation and the inroads made in technological advancements, one of the greatest beneficiaries is the healthcare sector. Technology has been extensively applied in the provision of healthcare services to improve patient experience and safety, and to mitigate the increasing cost of accessing healthcare services [147]. Technology has been applied in healthcare for information and data 
collection, storage, and sharing through healthcare information technology (HIT). At the same time, it has been extensively used in the actual provision of services such as GPS tracked heart pacers, pagers, and mobile phones applications in healthcare services provision. More specifically, application of technology in healthcare services provision has been successful in what is referred to as remote patient monitoring.

\subsubsection{Remote Patient Monitoring (RPM)}

Remote patient monitoring (RPM) is one of teleconsultation applications in the provision of healthcare services which involves the use of digital technology to collect, store, analyze and use health data collected from patients from remote locations to facilitate provision of healthcare services securely from a remote location [148, 149]. In this regard, the key elements of a remote patient monitoring system are the ability to collect, store, analyze, and transmit health data securely to facilitate provision of healthcare services from remote locations without the necessity of patients having to visit the physical facilities of the healthcare services providers $[150,151]$.

Almost everybody is happy with the introduction of the RPM in various hospitals. The RPM device is one that allows patients and doctors to share and exchange information mostly through devices like phones. The system is using digital technologies to collect an individual's health data from a specified location and transmit it electrically to the physicians. The usage of this scheme started years ago in a cardiac monitoring form. Doctors were managing the rhythm of the cardiac and heart failure using this system in the past and right now people have 
seen several changes in the system. According to Pekmezaris most of the individuals who are using this system are at home far away from the hospitals but are using the telehealth to communicate with doctors [152]. With the help of technology, doctors are accessing the medical device of a patient and can share it with other staff members anytime. Patients who are using the RPM system are under quality healthcare and medical condition monitoring from various doctors, and they are under a good observation at all times. Through the use of RPM, the chances of medical emergencies are reducing, and most patients are not coming back for readmission.

The RPM system is allowing a continuous connection of doctors and their patients and with such a system, doctors may have enough preparation time before treating a patient. They are having enough time for decision making before carrying out their duties, and during this period, they can consult each other on most of the issues. Before the introduction of RPM, people had less medical knowledge and support but right now patients are having a broad health education, support, and instant feedback to whatever question they are asking the doctors. Their health outcome is improving, and individuals can see a significant differentiation between the traditional healthcare models and the RPM technology system. Patients are getting daily and updated information that allows them to maintain good health.

Logan posit that data collected from patients through a point of care system includes but is not limited to vital signs, blood sugar, oxygen levels, heart rate, 
temperature, electrocardiograms, and weight of the patients [153]. Taking all these into consideration, an RPM system can therefore be defined as the collection of health data from a patient through the use of digital technological devices, transmission, storage, analysis, and utilization of the findings to provide healthcare services to patients. At the same time, it entails ensuring that the security of the whole systems is upheld to ensure patients safety, privacy, confidentiality and at the same time to guarantee the integrity of the system. Therefore, there are five main component steps of the RPM system, which are collect, transmit, evaluate, notify, and intervene.

The RPM system is benefiting clinicians in several ways. For instance, they are easily accessing the data of a patient, and this is allowing them to have the ability to deliver higher quality care to several people who have lower burnout risks. They are reducing the cost of medication and efficiently carrying out their duties hence quickly assisting the patients. The quality of care to the patients is improving due to system use, and this is allowing them to have peace of mind since their health is properly monitored. In the end, they are staying healthy at all times. Patients who are engaging with RPM are using it in controlling their health, and they are following and embracing any recommendation that caregivers are providing, hence improving the prospects of their health.

The RPM is benefiting the elder people since it is giving more efficient health monitoring to them, leading to safer lives. Most of the older adults have the chronic health conditions, and this is a risk top for their families and to the society as a 
whole. Therefore, the introduction of the RPM technology system is a way of helping these people with their problems

\subsubsection{How Remote Patient Monitoring Works}

As discussed above, a remote patient monitoring system involves application of digital technologies in provision of healthcare services. There is extensive use of wireless and wired devices in these functionalities. The wireless devices use a variety of methods to enhance connectivity to facilitate real time monitoring of patients remotely. These technologies include radio signal, Bluetooth connectivity, and other wireless connectivity systems and signals [154]. Therefore, a remote patient monitoring system is made up of three main components, the patient side terminal, the medium and the healthcare institution side terminal [155]. Premised on this, the architecture for a typical RPM system is made up of a sensing device on the patient site to measure vital signs and other physiological parameters, the patient site data storage device, a database to collect and store data from sensors, diagnostic applications from healthcare services providers, and healthcare services provider side interface running diagnostic applications that make recommendations and alerts premised on the data collected, to be stored and transmitted from the patients side as shown in figure $5[148,155,156]$. The healthcare site interface is manned by qualified medical personnel. 


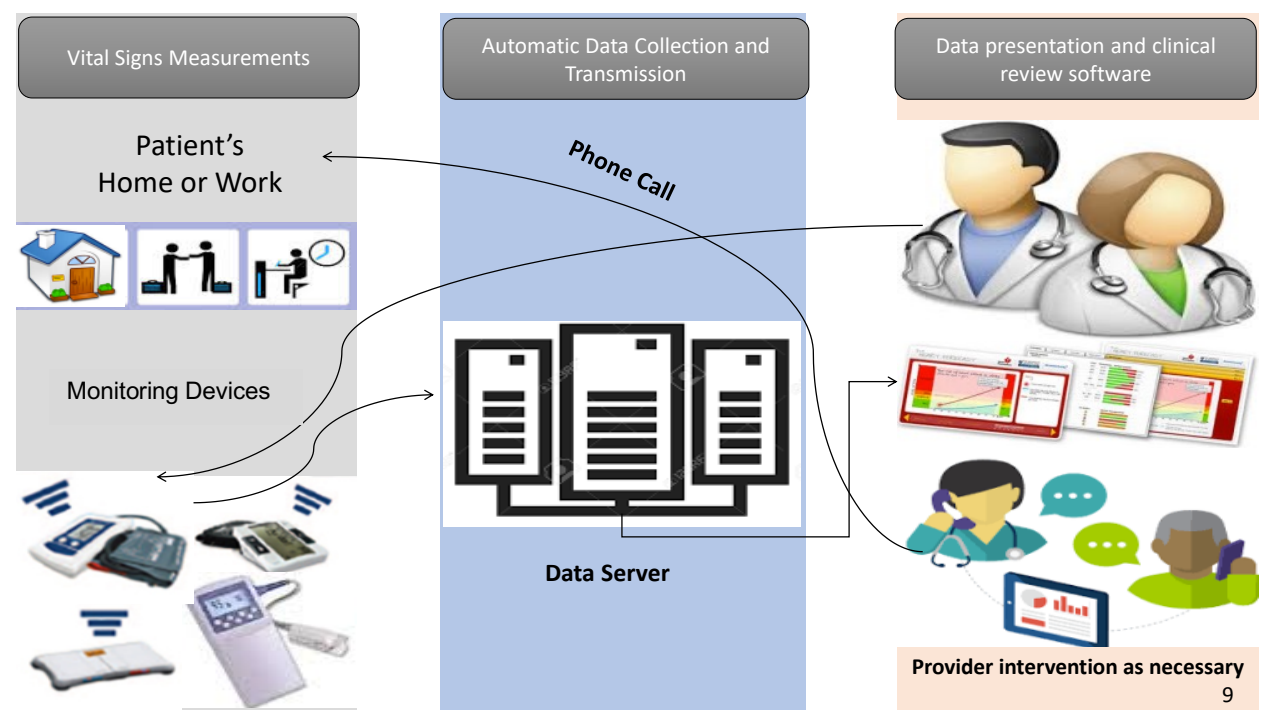

Figure 5 : How Remote Patient Monitoring (RPM) works.

When the data is collected from the patient's side by the sensor device such as a pacemaker, it is then stored in a local storage device on the patient side and processed by in-built applications. This is then transmitted to a central storage repository on the providers' site which is analyzed, stored, and interpreted, and used to form the basis for making recommendations on medication, or is used to raise alerts when the patient is in critical condition. The information then elicits immediate action by the healthcare service providers, who respond accordingly. The system also incorporates educational materials dissemination to act as a guide for a patient to live a healthy lifestyle [150].

Wide ranges of devices are used in remote patient monitoring. They include computers with customized software applications, telehealth response watches, pacemakers, prothrombin time/international normalized ratio (PT/INR) meters, blood pressure cuffs, thermometers, scales, glucose monitors, pulse oximeters, 
electrocardiogram ECGs, peak flow meters, stethoscopes, pedometers, and other technologies as shown in figure 6 [155].

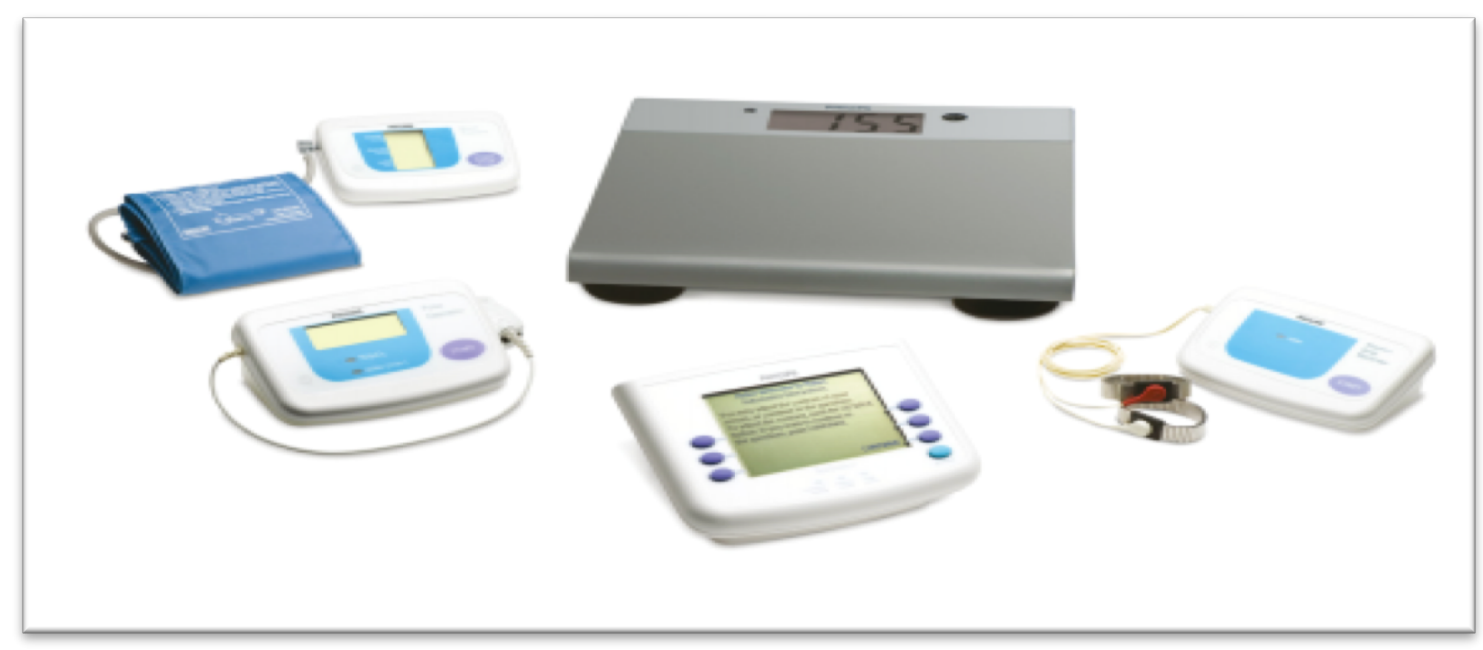

Figure 6 : Some Remote Patient Monitoring (RPM) devices.

(Source: http://www.healthcare.philips.com)

These devices, when interlinked and customized to a specific patient, enable the collection, transmission, storage, analysis, and response or intervention that is the essence or the basis for an effective remote patient monitoring system.

On a daily or a continuous basis, these devices will prompt the patient or the caregiver to input data or information to facilitate the monitoring and response system through a series of preset questions, some manually input or auto input data from peripheral devices, or through engaging care givers through video conferencing or uplinks to provide audio and visual support to facilitate clinical assistance from the healthcare services providers. This integrates all the devices and gadgets used into a central system that ensures that comprehensive data is accessed so that the patient can get comprehensive and customized services that are specific to their healthcare needs. Some of the systems are activated by the 
patient, others are activated by the caregivers, while still others self-activate to monitor and collect physiological information and or data on the patients' health condition or in relation to a specific condition that they are designed to monitor such as diabetes, congestive heart failure, infertility, dementia, and a number of other health conditions [150].

\subsubsection{Advantages of a Remote Patient Monitoring (RPM) System}

Raj posit that technology application in any field is designed to increase efficiency, effectiveness and productivity [155]. In healthcare, RPM systems and technologies have been instrumental in improving the quality of healthcare services due to their holistic and integrative approach to healthcare services provision. Patients, healthcare professionals, and the healthcare services system in general, have realized immense benefits from the utilization of technology to deliver comprehensive healthcare services remotely, that is, preventive and curative services. The RPM systems and technologies have played an important role in chronic disease management ensuring that patients live longer and healthier lives [148, 154].

First and foremost, RPM technologies and systems have enabled early detection of onset and deterioration which facilitates early intervention and in so doing, reduces the number of emergency department visits and hospitalizations. This has resulted in a reduction in the total number of days spent in hospitals by patients suffering from chronic and non-chronic diseases [154]. Early detection and intervention eliminate the need for unscheduled and preventable services that lead to a reduction of costs incurred by the patient and healthcare services providers. 
The incorporation of home health monitoring (HHM) into RPM enables caregivers to provide services to patients off site or in their homes or workplaces which leads to patients having better, more fulfilled lives since they are not tied down to a hospital setting $[147,156]$.

Secondly, RPM systems and technologies promote and enhance collaboration between healthcare providers, patients, and home-based caregivers. This ultimately promotes the quality of services delivered to patients. The system enables real time collection and dissemination of information which enables real time sharing of information across multiple providers, co-morbidities, and complex disease states. This integration increases collaboration, shared learning, and best practices in line with adoption of evidence based practices (EBPs) which promotes efficiency, effectiveness, productivity and quality of healthcare services delivered to patients [156].

As a result of information and communication technology, and the resulting technology systems in healthcare information technology (HIT) such as RPM, there is increased productivity from the fact that the element of time is eliminated by real time data transmission across multiple time zones. In addition, there is a decrease in the number of home visits for healthcare professionals in the number of unnecessary hospital visits for the patients [148]. At the same time, the use of these systems enables automated documentation and record keeping which eliminates tedious and time-consuming paper work. This results in improved efficiency and increased productivity due to the fact that from a remote location, a practitioner can deliver care to multiple patients at the same time in real time [153]. 
On the other hand, RPM and HHM systems as components of healthcare information technological systems involves the incorporation of patient participation in service delivery due to the fact that on the patients side terminal, the patient has a participative role in handling the equipment and devices used in the provision of services, giving the patient a high level of control and input into making decisions as to what services they access to promote their health [156]. This also results in increased patient confidence and trust in the healthcare systems due to the fact that they are actively involved in the decision making process regarding what healthcare services they receive to address their own health conditions [150].

The utilization of healthcare information technology, and more specifically remote patient monitoring and home health monitoring, has led to a reduced cost of provision and accessing healthcare services [147, 155]. This is because less money is spent in visiting homes for the physicians or the patients visiting the hospital. This result in the release of resources such as social workers, medical assistants, and community health workers to engage in delivery of services to other patients, which increases the quality of healthcare services. However, to ensure this, utmost security should be upheld because such systems are susceptible to violations such as unauthorized access, use and sharing of information which compromises the safety of the patient confidentiality and privacy of the data so collected [154].

One of the most reviewed success stories is the implementation of HIT; more specifically a remote patient monitoring system that incorporates the use of 
HHM in the Department of Veteran Affairs' healthcare information and management system $[150,157]$. The Care Coordination/Home Telehealth (CCHT) introduced by the Veterans Health Administration (VHA) has enabled the department to reduce the necessity of long term admission by utilization of remote patient monitoring technologies for veterans with chronic health conditions [157].

Health Information Technology, and more specifically remote patient monitoring, is an example of how technology can be extensively utilized to bring down the cost of healthcare services and at the same time improve quality of services and care delivery. Further, these approaches also increase the levels of trust in the healthcare system which helps mitigate the trust crisis, which is a major concern for healthcare management in both private and public sectors. However, it is important to ensure security of the systems to guarantee security, confidentiality, and privacy of the patient and the information collected using these systems.

Before the introduction of technology in healthcare, an individual could go to the hospital from treatment, and after getting medication, the data containing their health information could get lost immediately. In any case, when he patient returned, and it was tough to trace their records or data. This is no longer happening due to technology. The RPM system is workable due to the options and streaming for wireless connectivity like Wi-Fi. The wireless size matters a lot in the system of RPM since the connectivity range and speed depends on the size. The RPM is using the human factor engineering in order to work efficiently. The human factor engineering is the designing of an interface of human machines that is 
focused on the interaction of devices and individuals. An efficient RPM system contains privacy security when connected to any health apparatus. The RPM is providing safety and privacy to data in the devices and manages its solution in any case of device hacking. The system is identifying users, and in any event of a wrong one, they are giving alerts.

\subsubsection{Remote Patient Monitoring (RPM) for older patients}

The majority of people who are benefiting from the RPM are older individuals who are 65 years and above. These people sometimes have health issues, and hence need a close monitoring and immediate attendant in case of emergency. Most of them are suffering from dementia and falls, diabetes, asthma, heart failure, and strength loss among other diseases. Before the introduction of technology to healthcare, families with older adults had a hard time because they

were to keep an eye on them at every moment. Such situations no longer a problem, and these families are at peace without stress due to the monitoring devices. Nurses are no longer traveling to residences to give care to the elders. This paper is looking at the benefits that seniors are gaining from RPM technology.

\subsubsection{RPM Technology that is helping in arthritis control}

Arthritis is a disease that causes pain in the joints due to the inflammation; it is common in people who are 65 and above. The reason why this disease is common in older people is that they are no longer doing physical exercise or activities to their bodies. The condition is discouraging these individuals group from being active, hence experiencing pain and lower life quality. The introduction of 
remote monitoring of rheumatoid arthritis (REMORA) is helping those who are suffering from this disease. The technology is using a mobile app that is daily surveying the condition of a person. The seniors are engaging themselves with the app system, and they have easy time familiarizing themselves with this technology. Physicians are using the app to continuously provide the follow up care to those who are suffering from this illness [158]. They are monitoring these patients and giving them a continuous care through the mobile phones, and this is benefiting the patients.

\subsubsection{Controlling chronic diseases using the RPM System}

Chronic diseases are mostly afflicting the seniors, and sometimes causing death or disability; therefore, they need aged care everywhere they are. Patients with these chronic diseases are currently benefiting from the RPM system, and they are getting support while they are at home [159]. Most hospitals that have the program of chronic disease management are using RPM to manage patients' conditions find it to be critical [160]. The technology is allowing physicians to check in on the older adults as they are going about their lives daily. In emergency cases, the RPM is giving early warning to the doctors, enabling them to intervene, hence saving patients' lives immediately. Sometimes these older people are using telehealth to help in their communication with the doctors. Since the patients are old and they do not like going to the hospitals, the chronic disease management program is reducing and preventing hospitalization incidents. With the help of RPM, older people are feeling that home is the center care place, hence they are comfortable living and knowing that their lives are very safe. The rate of chronic 
diseases is reduced due to this system of connecting care and is prolonging the life of older adults living in the United States.

Telemedicine devices are increasing the autonomy of the older adults which increases their life quality. The technology of telecare is mainly focusing on those who are 65 and above years because their lives are at a significant risk [161]. The introduction of the smart home system is allowing these individuals to live in their choice of environment, and it is protecting them from nursing placement or institutionalization. The system is reassuring safety to these seniors, and it is a promising approach to independently living in a community. The solution of telecare is granting opportunities that are benefiting the lives of the older population. For instance, elder patients are getting the new diagnoses, education, and treatment opportunities, and those with chronic diseases are under a doctors' monitoring [162]. The smart home system is giving the opportunity to every senior, including those who are living in rural areas.

Doctors are assuring the older adults of their lives, and wherever a patient is, they know that someone is watching out for their wellbeing and health. The hospitals can install some of these RPM devices in the homes of the older people and watch them over while in their offices. Seniors sometimes cannot go for daily checkup appointments in the doctors' offices, yet when they have the devices at home, they are avoiding office visits and still taking good care of themselves as instructed by the physicians. Once the RPM is at home, the cost of medication is reduced, which allows them to save their financially; the encounters of the 
unneeded patient are reducing. The elder adults who are not financially stable can still access the system since the cost is low.

Seniors are relying on the doctors to give them information about therapy that they are carrying out daily. Technology is allowing them to receive the information while at home and this is improving their health lives. They are asking questions about their health and consulting doctors if they are ill. The physicians can give directions and instructions to those who are around the elder adults in any case they have an emergency through the RPM system. Doctors are monitoring the elder people who are not hospital friendly while at home. With the use of RPM, doctors are not only attending to acute conditions, but to everyone who is in the system, and this is improving the health management of elder adults who are chronically ill. Those senior individuals who know how to use the technology are more advantaged than those who do not know. They can visit the hospital portals, chat freely with the doctors without any help, and maintain the RPM devices on them, hence prolonging the use of this technology.

Since most diseases are targeting the seniors living in the community, they need to have education about the technology. Hospitals, together with the communities, should focus on training them and should introduce programs that are clearly explaining how to use the RPM technology. During the training, the trainers should clarify the technology advantages and its side effects in their lives. Doctors are recommending elderly patients to use the RPM since they need full attention and monitoring from them to avoid injuries; they should willingly participate in the remote monitoring. Monitoring the patient is a healthy activity, but 
prolonged use of an RPM system can lead to some disadvantages. For instance, if a patient cannot live without the technology because their lives are fully dependent on it and it fails, they may die.

\subsection{Successful Telemedicine Applications}

\subsubsection{Teleradiology}

The most conjoint existing applications of telemedicine are teleradiology. This involves the radiologic image conveyance within and among healthcare facilities. Numerous steps are characteristically convoluted in teleradiology comprising digitizing still pictures or openly generating cardinal photos, integrating demographic and other patient statistics, compacting images (data) in several ways to permit them to be conveyed more rapidly and economically, conveying imageries from one website to another, and reenacting images for broadcasting and understanding [41]. Extra steps are vital for the storage and retrieval of images automatically.

The progression of teleradiology implementations replicates numerous characteristics of radiology:

- Its well-recognized consulting substructure founded on correspondence and messenger services

- Its primary use of essential imaging equipment

- The accessibility of Medicare compensation for teleradiology consultations.

Radiology hubs have, for protracted periods, used transmitted or messenger-delivered pictures to offer, as designated by one association, 
"consultation, second estimations, and principal readings; image over-read (and other instructive and helpful services) for persons getting started in MR or other challenging modalities; quality control of image understanding; vacation analysis; and supplementary coverage for assemblies with an intensifying case volume as yet inadequate to validate contracting an extra radiologist" (UCSF, 1995).

In numerous circumstances, teleradiology can make such free amenities much faster and more expedient, and the automated storage of imageries lessens complications with misplaced or mislaid films as pictures move amongst or inside administrations. Radiologists can also have these images conveyed to home or office workplaces, so they may not have to go to the infirmary to see them when they are on call.

An additional use for teleradiology is the comparatively previous experience in radiology with the benefits and intricacies of computer-based digital equipment such as automated axial tomography and MRI. These were trailed by other equipment such as picture archiving, communication schemes, and progressive digital converting, which delivered the possibility of high-quality electronic diffusion of images. This progress made established implementation of digital radiology practicable and enabled the improvement of multi-institutional teleradiology systems $[42,43]$.

Predominantly precarious for the use of teleradiology is a third component: Medicare and other reportage guidelines that have permitted compensation for radiology consultations without the face-to-face collaboration essential for most 
other consultations. This prerequisite is a chief source of obstruction for many promoters of other telemedicine applications.

\subsubsection{Home Care}

The implementation of telemedicine in the home and other non-clinical settings demonstrates the importance of non-video means for offering statistics and guidance and for observing patient status. The most conversant non-video telemedicine selection is the use of the telephone. Doctors, nurses, and other staffs regularly chat with patients and relatives-providing material, examining their status, and providing comfort-without the expenditure or inconvenience of a workplace appointment for the patient or a home-based visit for the health worker. To decrease preventable office appointments, numerous health plans have established consultative telephone programs, operated principally by nurses, to

offer patients data, valuations, and commendations for monotonous medical complications.

For therapeutic and other predicaments, the 911 scheme works from any telephone to put individuals in touch with correspondents who evaluate the nature of the crisis, send therapeutic or other help as designated, and offer medical directives when essential [43].

In addition to direct communications, preset telephone amenities are used in numerous ways. For instance, collaborative voice replies structures allow persons to induct calls and reply to chronicled inquiries using a touch-tone phone. Such schemes have been used to test computerized telephone transmission for 
hopelessness, with survey scores delivered to callers along with toll-free follow-up phone numbers [42].

\subsubsection{Health Care for Prisoners}

Recent studies are demonstrating the augmenting interest in the potential of telemedicine to offer improved access for prisoners to well-timed generalist and professional consultations and to lessen the expenditures and inconvenience connected with existing on-site and off-site provisions [44]. Colorado, North Carolina, and Texas are among the emergent states with functioning programs, and other states are in the process of considering or testing programs. The primary goal of prison telemedicine is to circumvent the significant costs of either transporting medical experts to jail (the costs of which are extraordinary partly owing to antagonistic working circumstances) or conveying the patient (the costs of which are great because at least two sentries and a state vehicle are needed for security).

In North Carolina, it is projected that the average prisoner conveyance price for medical facilities is over $\$ 700$ [45]. Also, convict programs are expected to decrease public anxiety about prisoner escapes, provide previous admission to care and better admission to subspecialty care, and supply recorded certification of amenities that may be valuable in litigations. Because penitentiary telemedicine programs are producing the comparatively large number of program settings, they offer substantial prospective for careful appraisals such as those assumed and premeditated by Texas Tech and the University of Texas Medical Branch at Galveston. 


\subsubsection{Rural Telepsychiatry}

One of the existing radiology programs that has moved beyond the experimental status is RODEO. It was founded in 1988 when public mental health programs in 13 Eastern Oregon regions prearranged the Eastern Oregon Human Services Consortium (EOHSC). In 1991, EOHSC was bestowed a three-year $(\$ 700,000)$ grant from the Office of Rural Health Policy $(\mathrm{ORHP})$ to validate the usage of telecommunications in conveying mental healthcare in eastern Oregon, a great rural area distant from numerous subordinate and tertiary medical assets [46].

On the experimental side, the telepsychiatry program is used for consultation, patient assessment, prescription management, and emergency response over a 24-hour psychiatric emergency facility. Organizational, instructive, and other applications comprise pre-admission, pre-discharge, and transmission reviews; recommitment trials; ongoing health occupations education; knowledge teaching for both customers and benefactors; peer interacting; and controlling video conferencing. Obtainable public facilities include a free video, a two-way audio, and a two-way compacted video/audio/data link.

\subsubsection{Postsurgical Intensive care in an Urban Nursing Home}

The postsurgical intensive care program industrialized by Stanford University Medical Center and nearby Lytton Gardens Health Care Center demonstrates an instance of a telemedicine implementation provoked by genuine creativity without national grant funding. This program grew out of deliberations introduced by Lytton Gardens, a skilled nursing and housing facility that offers a 
range of services and living provisions for average earning senior citizens. The CEO projected that telemedicine could be used to help in the faster and immediate discharge of intricate surgical cases from the health center to the nursing amenity.

The first experiment comprised liver transplant individuals, trailed by reconstructive artificial and vascular operation patients. The surgeons obtain improvement notes from the doctor and nurses at the nursing center, and they can inspect patients who are delivered to a room fortified with a special video camera (activated by an accredited practical nurse) and an auditory link that allow both visual examinations of healing wounds and discussion with the patient. Using the collaborating video link, the nursing home has also introduced some other consultations and is deliberating their use in home care.

\subsection{Current Trends in US Telemedicine}

Telemedicine technology has amplified, and the price of equipment has reduced in the past ten years, subsequent in an upsurge of telemedicine study projects and the upsurge in the scope of those schemes. The Telemedicine Information Exchange (1997) catalogs over 130 telemedicine investigation websites. The "4th Annual Telemedicine Program Review" recorded 80 dynamic telemedicine packages in 1996, in 38 federations and Washington, D.C., 8 of which

use only stock and send technology and 72 of which implement a two-way collaborating audio-visual technology. [47]. There are 1,032 perfect telemedicine places (centers and shafts), which accomplished 21,274 consultations, 91\% $(19,380)$ of which were communicating audio-visual and $9 \%(1,894)$ of which were stock and forward. 
Since 1993, when there were only 12 dynamic programs in the United States, the number of programs has doubled annually, while program action (amount of consultations) has tripled since 1995. The highest ranked 5 types of consults in 1996 were [47]:

- Mental health $(21 \%)$

- Trauma care $(16 \%)$

- Cardiology (12\%)

- Dermatology $(11 \%)$

- Surgery (8\%).

Alternative or trauma telemedicine materialized in 1996 as one of the fastest mounting applications of this expertise. Initial projects expanding telemedicine in countryside healthcare demonstrated highly advantageous effects on patient subsistence and reclamation, but the paraphernalia was affluent and rather burdensome [47]. As the price and size of the apparatus have declined, and the scientific value has also declined, telemedicine has become much more practical to implement in rural healthcare.

Telemedicine is changing the way we experience healthcare. Healthcare providers are becoming more interesting in the idea every day, especially when they realize telemedicine benefits: better care outcomes, less hospital readmissions, happier patients, and more profitable practices. Also, patients are starting to see and realize that telemedicine advantages such as accessible healthcare are more convenient. As a result, telemedicine is on the rise. According to the American Telemedicine Association (ATA) the use of telemedicine programs 
is increasing and more than $50 \%$ of all United States hospitals currently have a telemedicine program. According to Dr. Reed Tuckson, the president of ATA, there were about 800,000 online consultations in the United States in 2015 [48].

In September and October of 2014, "Foley" distributed a telemedicine survey to healthcare executives in the United States and 57 healthcare leaders from for-profit and nonprofit care providers were surveyed [49]. More than $80 \%$ of surveyed healthcare executives realized that the development of telemedicine services is either important or very important to their organizations as shown in Figure 7 . Only $1 \%$ of the surveyed healthcare executives felt that a telemedicine service is not important to their organizations.

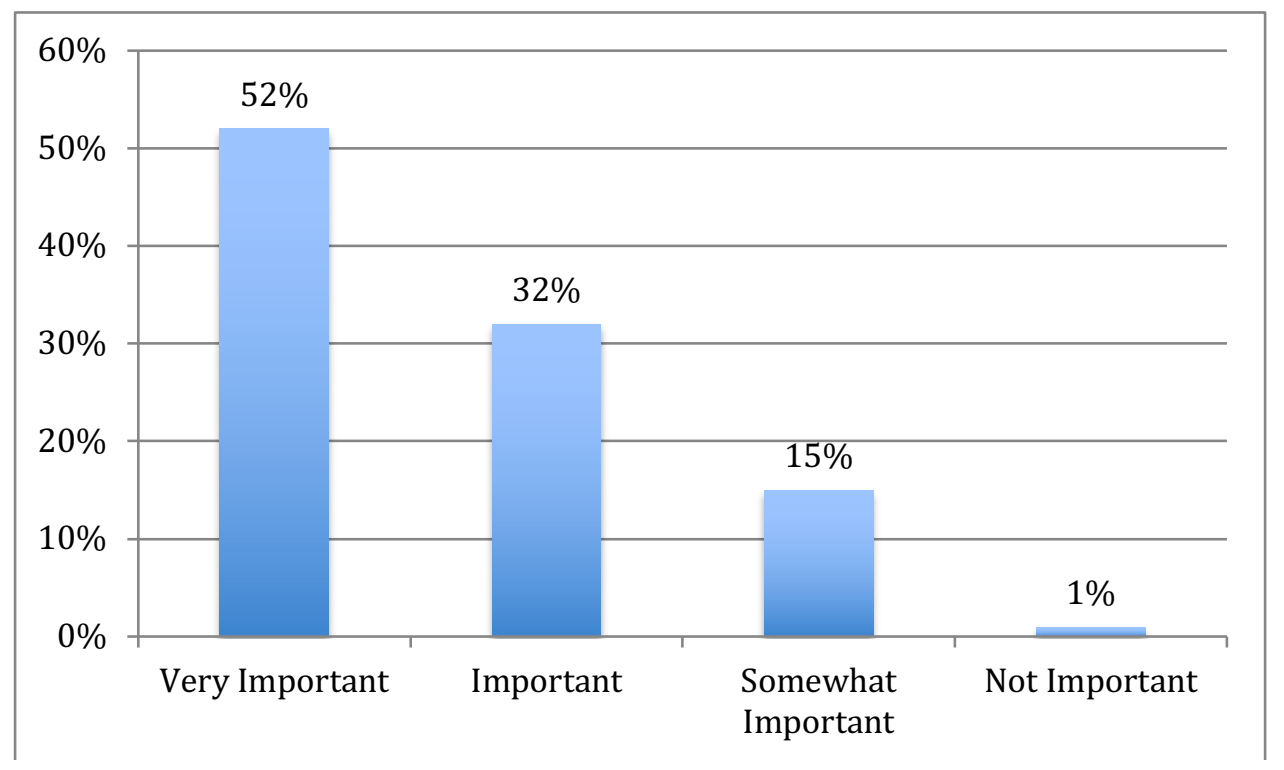

Figure 7 : Telemedince important to healthcare organization. (Source: Foley survey - 2014)

According to the same healthcare executives survey regarding the top telemedince practices currently available to patients through their healthcare 
organization, remote monitoring ranked the top practice by $64 \%$ followed by store and forward (54\%) and real time capabilities (52\%) as shown in Figure 8 [49].

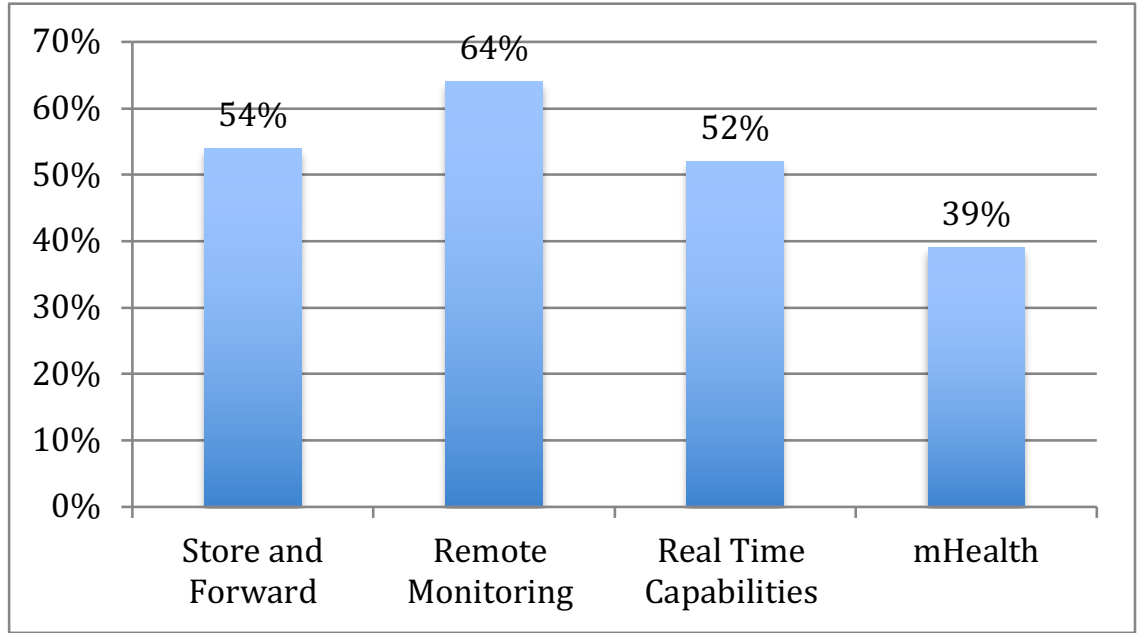

Figure 8 : Top telemedince practices currently available to patients.

(Source: Foley survey - 2014)

The same experts were answering questions regarding the current status of their telemedicine program in their organization and more than $90 \%$ reported that their organizations have already begun developing or implementing a telemedicine program as shown in Figure 9. However, telemedicine programs were in the earliest stages and about $50 \%$ of the experts reported that they were still in the development phase (34\%) or in the pilot phase (16\%) [49]. 


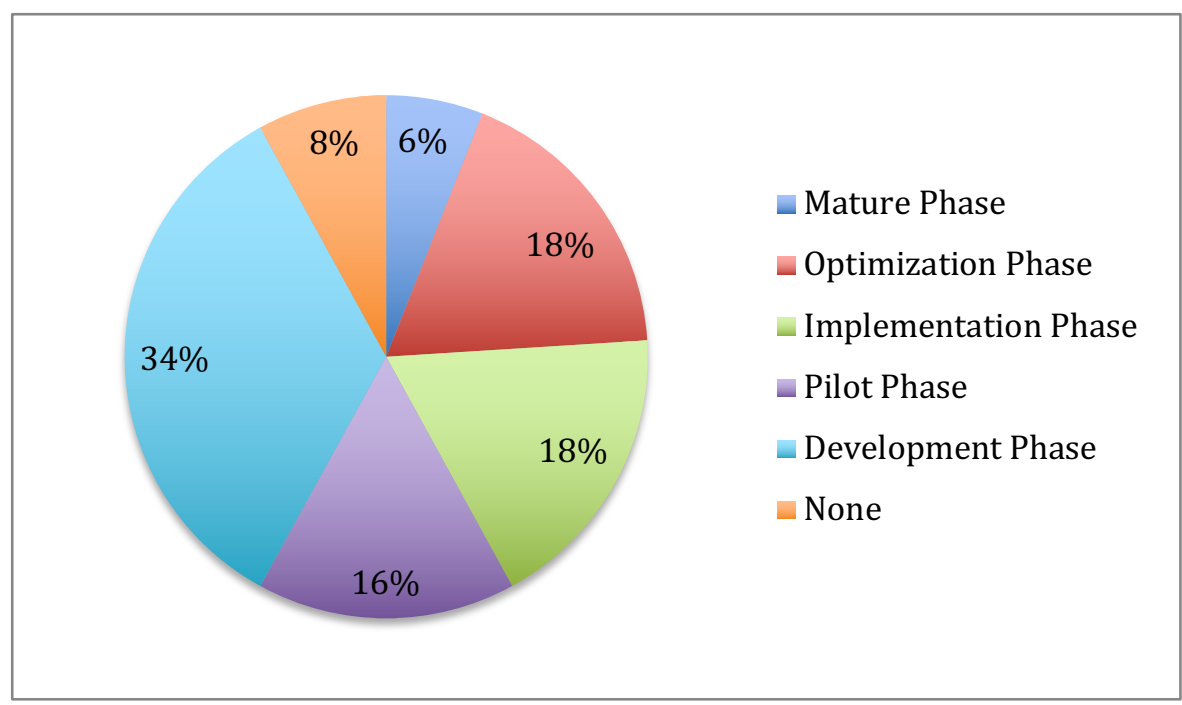

Figure 9: Current status of telemedince program. (Source: Foley survey - 2014)

In the RNCOS' latest research study, "Global Telemedicine Market Outlook 2020", the analysts have studied the whole telemedicine industry and according to their analysis, the market for telemedicine technologies, which include hardware, software and services, was almost 18 billion in 2014, and is projected to grow from 2014 to 2020 at a compound annual growth rate (CAGR) of 18.4\% [50]. Another market study expected the telemedicine market would reach 20 billion by 2019 and that almost $20 \%$ of the whole health IT market.

The global revenue forecast telehealth devices and services market from 2013 to 2018 and the worldwide revenue for telehealth devices and services is expected to increase from $\$ 440.6$ million in 2013 to $\$ 4.5$ billion in 2018 , (based on data from an IHS report) as shown in Figure 10. 


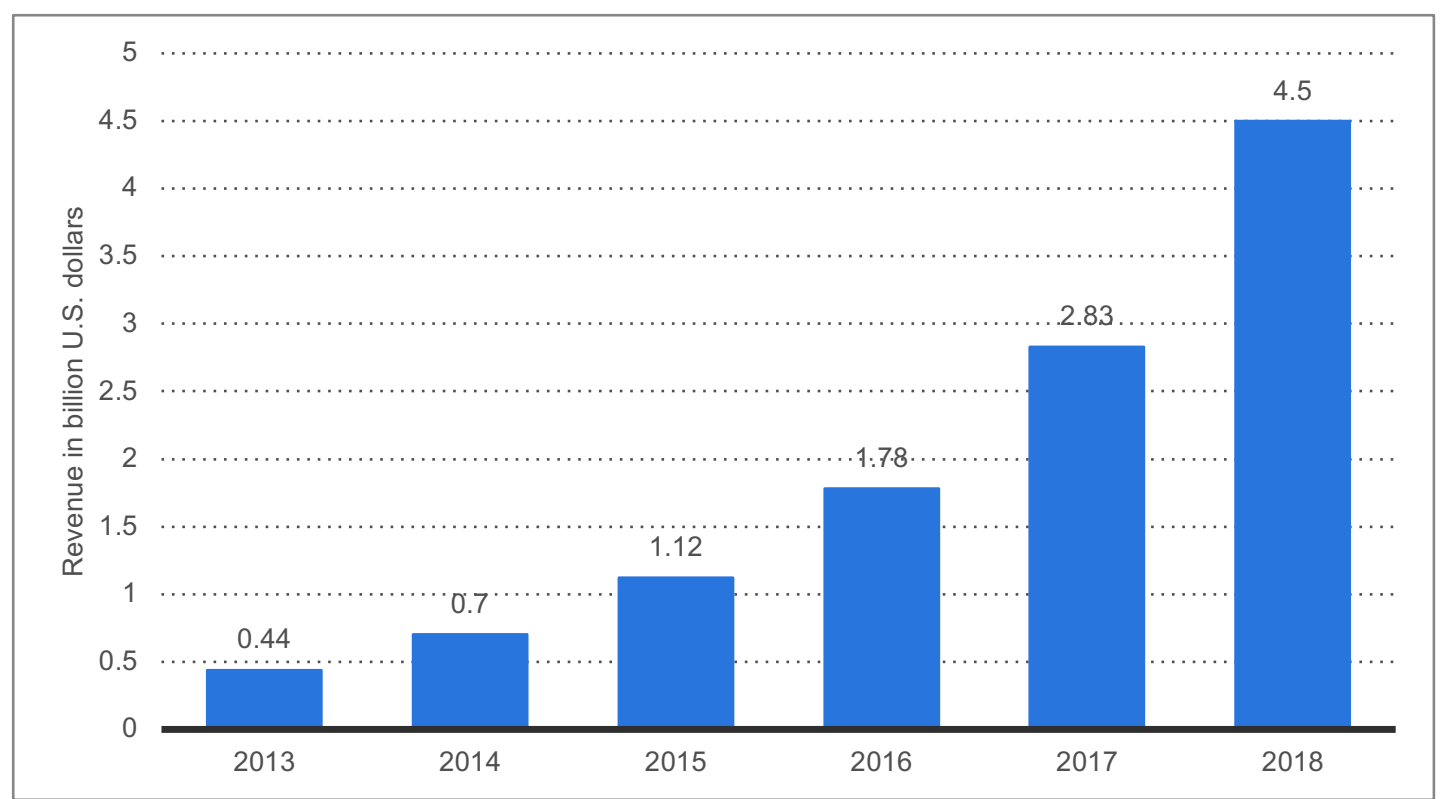

Figure 10 : Worldwide revenue telehealth devices and services 2013-2018.

(Source: IHS report).

Projected number of telehealth patients worldwide from 2013 to 2018 (in millions) The number of patients using telemedicine services will reach about 7 million in 2018, comparing to less than 350,000 in 2013 as shown in Figure 11.

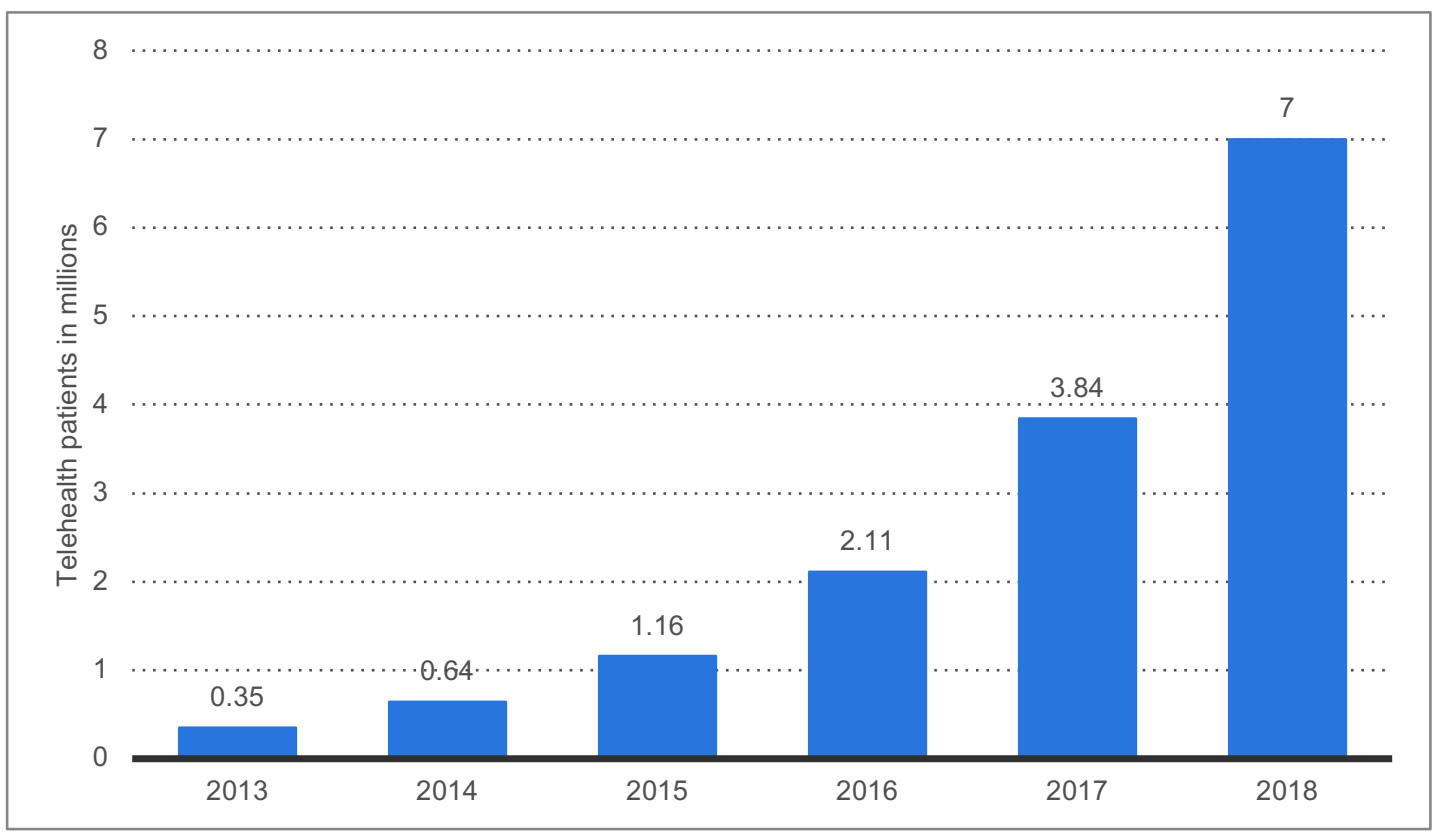

Figure 11 : Forecasted number of telehealth patients worldwide 2013-2018. (Source: IHS report) 


\subsection{Benefits of Telemedicine}

Telemedicine has numerous benefits for patients, providers and the economy. The demand for telemedicine has continued to increase for the patients and as well as the providers. Patients like telemedicine for the two main reasonstime saved and convenience. On the other hand, providers like telemedicine for easier monitoring and delivery of earlier treatment. In general, this will improve the healthcare system and reduce the cost of the treatment.

\subsubsection{Patients}

There are many benefits of using telemedicine by patients, some of these benefits relate to time, money, and quality of service. With the classical healthcare system, like any service delivery system, clients travel to the service provider seeking service. In this case patients have to physically travel from their rural areas to metropolitan areas in order to consult with a medical specialist. By using telemedicine, patients gain the consulting they want from their home or the rural primary care provider facilities. As we know the United States has large rural areas, and it is clear patients have an opportunity to save millions spent annually on vehicle travel expenses.

Many patients prefer to stay in their home as they can, before they go to a healthcare provider. Also, as part of reducing the healthcare services costs many patients are leaving the hospital earlier and taking their additional care at home while they recover because treating patients at home is less expensive as in the hospital. According to a new research in-home care nurses found telehome care 
minimized the nurses visiting time and reduced the cost $33-50 \%$ from the traditional home care visit.

Quality is one of the advantages of the telemedicine, where patients in rural areas can access high caliber healthcare services. According to one study about $20 \%$ of the United States population (55 million) reside in rural areas and as citizens they also deserve the same healthcare quality services as the other $80 \%$ who reside in metropolitan areas. Telemedicine will improve the healthcare quality by increasing the collaboration between providers in order to enhance the services offered to patients.

\subsubsection{Providers}

By applying telemedicine providers can gain many benefits such as access to information, reduced medical errors, and increased working efficiency. In business, time equals money, but in an emergency room time is life. Telemedicine provides immediate access to patient information and accurate medical topics at the same time.

Telemedicine improves the accuracy of diagnosis and reducing medical errors that is very important for the medical community. The simplest practice is "tele-assistance" where a physician acquires either a second or specialist's opinion on his/her patient diagnosis. Diagnosing correctly at the first time has many benefits for both patients and hospitals, reducing the average recovery time and using less unneeded medicine for a reduction of costs to patients and hospitals. 
Another benefit of telemedicine is the reduced travel time for both patients and doctors and leading to the increase in efficiency. It will decrease the use of paperwork and research time of medical records. According to the United States Army, telemedicine speeds up the whole process in the foreign military bases. They consult from anywhere and reduce the decision-making time.

Continuing education is very important in any field and it is essential in healthcare. Telemedicine can enhance providers' education and keeping them up to date about medical topics. Physicians can improve their educations with the latest knowledge in their offices, saving time and money.

\subsubsection{Economics}

The local economy benefits by applying telemedicine, increasing business retention and recruitment. Telemedicine can improve the healthcare services delivery system. The new telemedicine technologies increase the home health providers' efficiency by reducing the travel time to patients' homes. Patients have more opportunity to be seen by specialists while using new telemedicine technologies.

Healthcare is very expensive, especially in rural areas where healthcare spending has a major impact on the economy. As telemedicine reduces non-local spending, the local economy is enhanced by spending local currency. Telemedicine can increase the ability to provide healthcare services to rural areas. In general, this capability will increase the retention and recruitment in the healthcare sector and especially in the rural areas. 
In rural areas some people cannot travel outside the community for training or studying. Telemedicine can provide high quality healthcare education and training partnerships with educational institutions by using videoconferencing in rural communities. This can help cover the shortage of medical staff in rural hospitals by hiring more local people. Telemedicine provides quality healthcare services for people in both metropolitan and rural areas. 


\section{CHAPTER 3: RESEARCH APPROACH AND METHODOLOGY}

\subsection{Research Objectives}

The research addresses the following objectives:

1) Provide a systematic approach for comprehensive assessment of teleconsultation adoption.

2) Develop a decision model to assess the effectiveness of telemedicine policy targeted to increase teleconsultation adoption.

3) Evaluate and prioritize current teleconsultation adoption barriers and measure their relative contribution to this adoption.

4) Evaluate and prioritize the alternative solutions and measure their relative contribution to this adoption.

\subsection{Research Questions}

The importance of telemedicine and its applications in the healthcare industry are well recognized. From all that has been previously discussed, it becomes imperative to increase empirical research in order to better understand the barriers that affect the adoption and use of teleconsultation applications. The following 16 research questions have been identified in order to address these gaps.

1) How do perceived usefulness and perceived ease of use impact the diffusion of teleconsultation?

2) Which barriers have more effect on the adoption through perceived usefulness? 
3) Which barriers have more effect on the adoption through perceived ease of use?

4) Which financial barriers have more effect on the diffusion of teleconsultation?

5) Which technical barriers have more effect on the diffusion of teleconsultation?

6) Which logistical barriers have more effect on the diffusion of teleconsultation?

7) Which ethical barriers have more effect on the diffusion of teleconsultation?

8) Which culture barriers have more effect on the diffusion of teleconsultation?

9) How does government support help the diffusion of teleconsultation?

10) How does training help the diffusion of teleconsultation?

11) How does multi-state license help the diffusion of teleconsultation?

12) How do telemedicine guidelines help the diffusion of teleconsultation?

13) How do multi-state standards and regulations help the diffusion of teleconsultation?

14) How do reimbursement standard processes help the diffusion of teleconsultation?

15) How do ethical codes help the diffusion of teleconsultation?

16) How does education help the diffusion of teleconsultation? 
Three literature review gaps have been identified, sixteen research questions have been developed, and the research objectives have been established. The literature review, research questions, and research objectives are summarized in Figure 12.

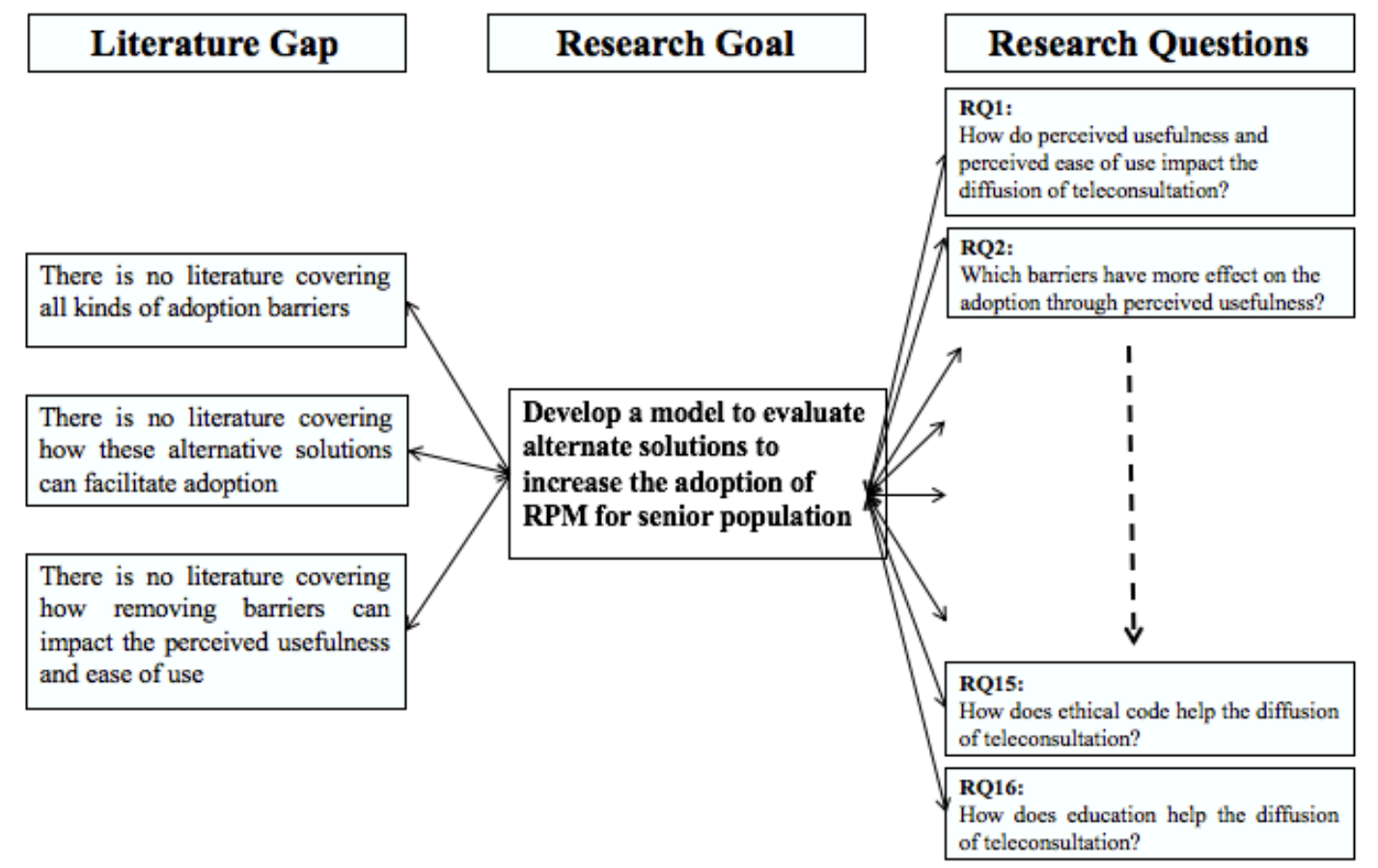

Figure 12 : Literature review, goal and questions.

\subsection{Multicriteria Decision Making Methods}

Multi criteria decision making is a unique tool that can be applied in many complex decisions. People make many decisions per day, although this same procedure is applicable in crucial aspects of society such as government, corporations, and the medical world. A multi criteria decision analysis is equated to a cost-benefit analysis, although it is not related to monetary units as a way of comparison. Therefore, when making complicated and essential decisions, one 
needs to consider multiple criteria. Notably, comparing similar sets of criteria can, at times, lead to a lack of clarity and accountability.

There are many multi-criteria decision methods, which depending on expert opinion such as:

- Analytic Hierarchy Process (AHP): It is a decision-making method which developed by Thomas Saaty. AHP is a well-known technique that has been applied in academia and industry to select among competing alternatives in a multi- objective environment and others. It is designed based on the welldefined mathematical structure of consistent matrices and ability to generate true or approximate weights. The method converts individual opinion into ratio-scale weights that are combined into linear additive weights for the associated alternatives. The final weights are used to rank and select the best alternative or option. The judgments are made in a pairwise process. The AHP method is flexible, intuitive and checks inconsistencies [164].

- Analytic Network Process (ANP): This method is more general form of the AHP used in multi-criteria decision analysis. The ANP structures as a network, while AHP designed a mission as a decision problem into a hierarchy with a goal, decision criteria, and alternatives. Both methods use pairwise comparisons to weight the components of the structure and select the best alternative based on the expert's opinion. ANP prediction process is accurate because feedback improves priorities [164]. 
- Technique for the Order of Prioritization (TOPSIS): It is a simple ranking method application developed by Hwang and Yoon in 1981 which is an extension of the theory of ideal solutions developed by Zelen in 1974. The chosen alternative should have the shortest distance from the ideal solution, while it should have the farthest distance from the negative-ideal solution. The positive ideal solution leads to maximize the benefit criteria and minimizes the cost criteria, whereas the negative ideal solution is vice versa. TOPSIS is a technique that combines quantitative attribute such as price, time, distance, and soon; and qualitative attributes such as quality of the relationship, quality of assurance, reliability; and compares all alternatives together based on these attributes. TOPSIS provides alternatives ranking, makes full use of attribute information, and does not require attribute preferences to be independent. Fuzzy TOPSIS method is used to eliminate an unreasonably large number of pairwise comparisons and to solve MCDM problems [164].

- Simple Additive Weighting (SAW): This method is well-known and most widely used MADM method. SAW method is also known as the scoring method which it is one of the best and simplest types of multiple attribute decision-making method. The fundamental rationale of the SAW method is to get a weighted total whole of performance ratings of each alternative overall attributes. SAW uses the expert opinion for criteria and alternatives weighting. However, this method is often argued by some researchers and 
practitioners because of the unbalanced scale of estimations that use during its process and its inappropriateness of crisp ratio representation.

A study conducted by Coban revealed how multi criteria decision making was applicable in Istanbul, Turkey, regarding municipal solid waste management. In modern day, solid waste management has become an essential issue for developing countries. This has been attributed to technological growth, increased population, and improved standards of living. Therefore, in such a complex environment, municipal councils and societies need to come up with the most effective solution to manage waste. Coban stated that various disposable techniques were used in Turkey. On top of this, he revealed that these techniques were also applicable to other countries in Europe and Asia. The aspect of a multi criteria decision presented itself in the aforementioned scenario since eight solid waste disposal scenarios were assessed through seven criteria which were subject to monitoring and approval by experts [51]. Coban stated that "Towards this end, three different multi-criteria decision-making methods, namely, Technique for Order Preference by Similarity to Ideal Solution (TOPSIS), Preference Ranking Organization Method for Enrichment Evaluations (PROMETHEE) I and PROMETHEE II were utilized for the scenario evaluation." Therefore, the aspect of multi criteria decision was widely seen from the aforementioned techniques.

Another study conducted by (Villacreses, Gaona \& Martínez-Gómez et al., 2017) demonstrated multi criteria decision whereby Ecuador was looking for a suitable place for locating the wind power plants by using the Geographical 
Information System (GIS) [52]. Villacreses revealed: "parameters (wind speed, air density), relief (slope), location (distances to substations, road network, urban areas, transmission lines, charging ports) and environmental parameters (vegetation coverage), have been considered." The aforementioned parameters created a choice of multi criteria decision whereby they discovered a suitable area for setting the wind power plants was in the Andean region. The four selected multi criteria decision-making methods yielded to the same result. Notably, the situation was a complex one since it needed a sophisticated decision of choosing a suitable location. Therefore, multi criteria decision making was helpful in identifying the appropriate area.

Multi criteria decision making has shown its prowess in reaching a suitable decision. Therefore, a study conducted by Sanchez-Lozano revealed that it could be used to select an ideal location for placing the wind farms [53]. In most instances, the criteria used to make use of a multi criteria decision is based on numerical values, although at times it can be qualitative. Therefore, Sanchez elaborated more about the fuzzy approaches of the multi criteria decision making and their role in assisting the selection of the onshore wind farm site selection. Senthil also elaborates how multi criteria decision making is used in risk prioritization in a reverse logistic network [54]. Such risks are assessed and analyzed using hybrid techniques. Tougher environmental laws and a decline in raw materials have insisted on the importance of using reverse logistics. Senthil 2018 says that "a substantial amount of risk is involved in the reverse supply chain which has to be managed by organizations effectively." Senthil's provides a 
roadmap of knowing how to use reverse logistics in risk prioritization. He goes on to state that risk prioritization is an essential feature of a multi criteria decision making problem. Therefore, the risks involved in such reverse logistics are given a higher preference when using the hybrid multi criteria decision. The results of the process revealed that the importance of managing the inventory has a huge effect when dealing with reverse logistics.

Multi criteria decision making has largely been used in coming up with a suitable solution with the purpose of adhering to the set policies. Therefore, a study conducted by Tahri showed how the multi criteria decision-making method was effective in Morocco when fostering the development of renewable technologies [55]. Tahri focused his work on assessing the combination of the geographical information systems and multi criteria decision-making technique. This was aimed at identifying a suitable location for setting up a renewable project. According to Tahri, "Four criteria were used: location, orography, land use and climate, and the Analytical Hierarchy Process (AHP) was used to calculate the corresponding criteria weights." Therefore, using the aforementioned criteria, climate was perceived to be the best criterion since it gave a suitable platform for knowing the potential electricity production of a specific photovoltaic field.

Another study conducted by Penadés-Plà revealed that "conventionally there is a strong relation between manufacturing and services in complex engineering industries." This way, he explained more about the competition in the manufacturing sector. They have to utilize the suitable decision making so that they can enhance their maintenance delivery. The aim of the authors was to 
evaluate the multi criteria decision-making models and evaluate the different models by comparing them from a maintenance management perspective [56]. The final methods are chosen based on the criteria. Penadés-Plà, takes into consideration the different models and creates a platform for selecting the best approach for maintenance management.

As mentioned earlier, the use of a multi criteria decision method is suitable for corporates and governments. Therefore, according to Yilidz, a firm may need to take advantage of the method when selecting the best suppliers [57]. It is a crucial process as it pinpoints the challenges associated with choosing a perfect supplier, choosing the right criteria, showing their interactions clearly, and showing usage. Yilidz goes ahead to state that they are useful criteria for solving problems associated with multi criteria decision methods. Considering the use of more than one criterion when choosing the appropriate supplier makes the selection process lose clarity. The study states that conventional methods cannot guarantee a realistic solution.

A study by Dozic revealed that the use of multi criteria decision method has been used even in the aviation sector [58]. Any decision made in the aviation industry is carried out with extreme precautions owing to the huge investments made. Dozic states that "decisions usually need to be made while considering different, very often opposing criteria that could be both quantitative and qualitative." Therefore, this implies that the use of a multi criteria decision making method will be helpful in such a situation since it will assist in the decision making process. This study focused on 166 research papers whereby all of them were 
reviewed from 2000 to 2018 . The papers were divided according to four crucial areas, which were airlines, airports, air traffic management, and others. Each of the aforementioned groups referred to a specific stakeholder in the aviation industry. On top of this, it is subdivided into identified themes in the papers that have been reviewed. The subdivisions are evaluation, selection, ranking, improving, and others. Additionally, the application sections were analyzed in a systematic manner and the results stated in the research paper. The results of the work revealed that a multi criteria dimension approach has been a choice suitable for airlines. Evaluation was found to be the most common theme, and fuzziness was encompassed in approximately $50 \%$ of the papers. For this reason, the study concluded that multi criteria decision making was the ideal approach for airlines.

\subsection{Hierarchical Decision Model}

The HDM structure was first used by Kocaoglu to solve complex multicriteria decision problems [59] \& [60]. For any hierarchical decision model, the basic structure of the hierarchy is presented in the Mission, Objectives, Goals, Strategies and Actions (MOGSA) form. This model consists of five levels of decision elements namely Mission, Objectives, Goals, Strategies and Actions as shown in Figure 13 [61]. The process requires the decision maker to provide judgments about the relative importance of each of the criteria and then to specify a preference for each decision alternative on each criterion [62] \& [63]. 


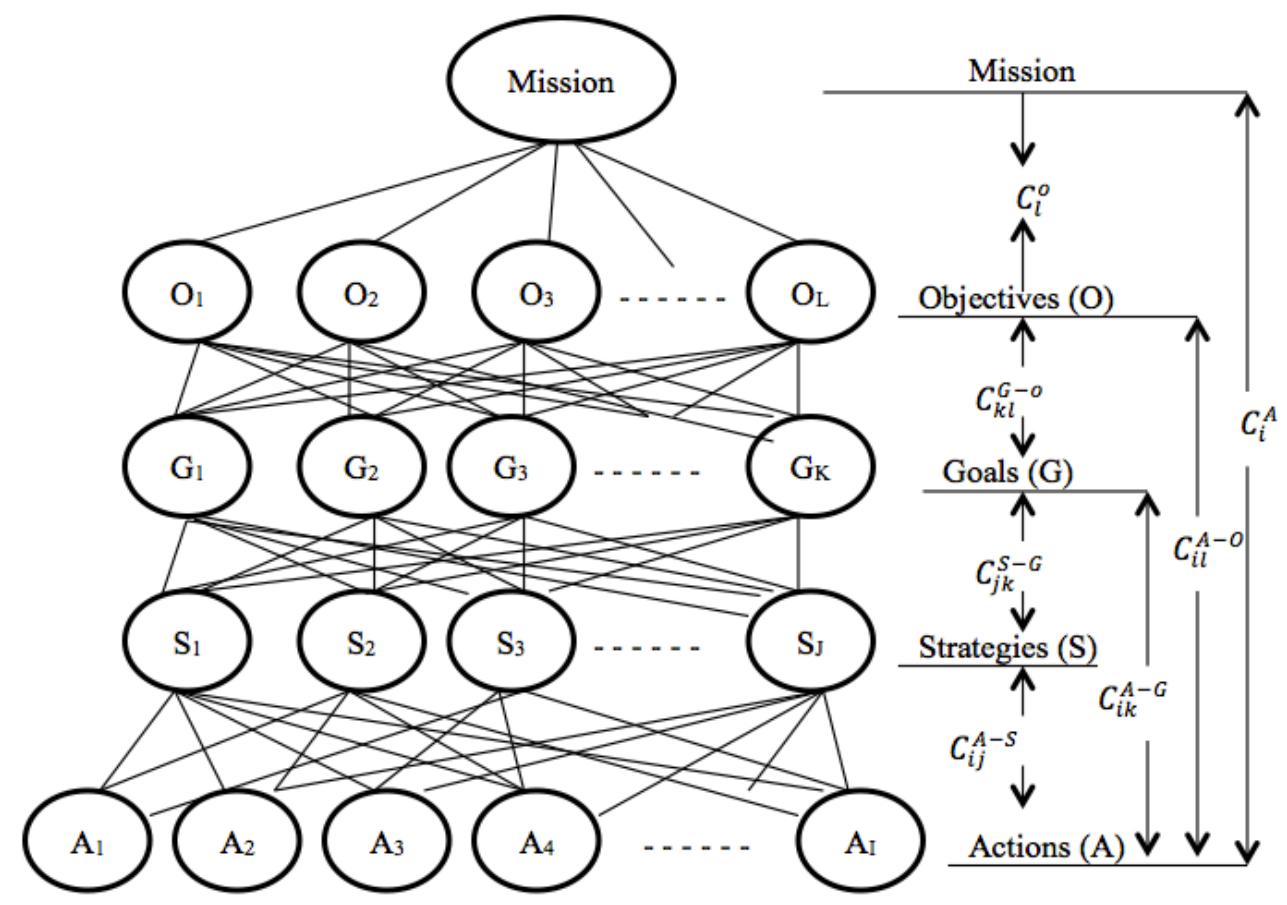

Figure 13 : Five levels of the Hierarchical Decision Model (HDM)

The hierarchical decision model is structured to solve complex decisionmaking problems by making use of qualitative and quantitative aspects during an evaluation process. In addition, the people in charge of making policies place the problems in hierarchy so that they can assess and evaluate which is the best decision to make and which will benefit their objectives. A study conducted by Sheikh showed how the HDM was suitable for selecting target markets for a new personal healthcare device [64]. This study elucidated that a new product was being launched by a multinational firm whereby it was meant to assist asthma patients with managing the problem by wearing the product as an ambulatory device. This device was meant for reducing exacerbations, which is a major contributor of asthma. However, introducing a new device in the market can be extremely challenging for medical equipment as the process is expensive and 
requires a rigorous approval process. Therefore, the firm opted to focus on one target market, and this was viewed as a cost-effective method instead of entering the entire medical market. Therefore, several niche markets were identified, and to find out the most attractive one, the HDM was identified as a way of addressing the problem. The solutions it provided was that asthma management and home care markets were the most efficient and lucrative markets. Therefore, Sheikh affirms that the HDM was beneficial in identifying the correct target market.

In contemporary society, the HDM is helpful in solving problems. This is affirmed in a study conducted by Gandhi to reveal how the model was used in solving traffic congestion problems [65]. The number of vehicles has increased at a tremendous rate and this is owed to engineering and technological advancements. For this reason, a need to create and manage a proper flow of traffic arises. This is for the sole purpose of maintaining a civilized nation. This study reveals that various techniques are used for traffic objection. However, it focuses on developing a suitable, convenient, and effective algorithm that can be used to control the traffic signal lights. For this reason, the HDM is put into play when the local decisions are provided based on the statistics and global decisions based on the pattern learnt at a higher level. Situations that arise such as the emergency arrivals and accidents would be taken care of by the global nodes network.

Another study conducted by Kim showed the use of the HDM when assessing arms import policies in South Korea [66]. The decision to import arms is a crucial one since it impacts nations in various ways. Priorities on diverse policies 
play a pivotal role in causing conflict among the policy makers. This is because the decisions involved are either qualitative or quantitative elements that arise from various quarters. For this reason, it becomes difficult to compare them and use them when making a final decision. When dealing with arms, another decision model is the rational decision model; however, the HDM is used to pinpoint the elements of the decision. Kim states that the Republic of Korea uses a hierarchical cluster analysis and MANOVA when handling disputes and altercations between the decision makers and the practitioners. He goes ahead to state that the stakeholders involved in the procurement of arms make their decisions based on the organizational missions and as a result, it affects their policy priorities. If not done well, it can lead to major disagreements.

Hu reveals "we explore using latent natural language instructions as an expressive and compositional representation of complex actions for hierarchical decision making." This implies that before they integrate the micro actions, the agent's first undertaking is to carry out plan in the natural language, which is then implemented by another different model [67]. The study focuses on an experiment whereby a dataset of 76000 pairs of instructions are gathered. In addition, the executions from human play, the training instructor, and the executor models reveal that the natural language of instruction supersedes other models since it is based on a hierarchical decision.

A study conducted by Arzola showed how the HDM can be pivotal in various industries [68]. Their study focused on a company known as eKnow, which is focused in the provision of high-speed internet in American schools. When the 
company was first launched, its focus and decisions were based on the opportunities and the connections in various states. Therefore, after having penetrated the different states, the decision making procedure is advanced. The company is focused on entering different states; therefore, this study was aimed at helping them find out the appropriate decision criteria and then use them in the HDM to identify which states were to be prioritized. The result was then used to assist the decision makers to make the appropriate choice to go forward.

Libya has undergone major transformations and changes whereby it has created suitable avenues for investment. Therefore, a study by Dabab showed that the country has massive positive elements that make it suitable for making investments in various sectors [69]. For this reason, the government was reaching out to potential investors who would offer the last updated technology, which would help the labor service. The ministry of telecommunications showed its willingness to find an investor who would invest in the long term to the cellular telecom sector; this sector was identified as one that needed reforms. To improves the chances of success, four options were stated. Dabab identified them as "privatization of the companies, licensing a new foreign operator, supporting existing operators, and joint venture." The aforementioned options were assessed and evaluated using the HDM. It was later concluded that the licensing of a new foreign operator was the best method.

Another study conducted by Lee elucidated more about the HDM [70]. This is where he focused his work on the role of social media in disaster management. In such circumstances, social media provides data on a large scale regarding what 
is happening. For instance, some applications are helpful in improving the efficiency of positioning the supplies and improve the efforts used in mitigating the situation. Therefore, to make maximum use of the apps, it is essential to have a robust human behavior model in the social networks, which creates a platform for individual decision making as well as interactions among individuals. Lee says that "we introduce a hierarchical human behavior model by associating extended Decision Field Theory (e-DFT) with the opinion formation and innovation diffusion models. Particularly, its expressiveness and validity are addressed in three ways. First, we estimate individual's choice patterns in social networks by deriving people's asymptotic choice probabilities within e-DFT. Second, by analyzing opinion formation models and innovation diffusion models in different types of social networks, the effects of neighbor's opinions on people and their interactions are demonstrated." Therefore, using the aforementioned approach, a proper framework is created for creating better disaster management strategies.

A study conducted by Carli showed how the hierarchal decision making strategy was useful in the energy management team of a smart city [71]. The initial decision strategy helps the people in charge follow the right channel by basing their decisions on another smart city. Therefore, to solve the problem of the hierarchal decision making, they based the situation on a case study in the city of Bari (Italy) since it has adopted a smart city program.

From the literature review it is apparent that the multi criteria decision making model has been helpful in many fronts, especially where complex decisions have to be made. Governments and corporates have found themselves 
on the tough side whereby they have to decide irrespective of the situation. For this reason, the multi criteria decision making model has shown to be helpful. In addition, the HDM has also been used although during an evaluation it considers both qualitative and quantitative aspects. Many people, governments and companies rely on it as a way of making crucial decisions. There are situations that arise that force many stakeholders to use the HDM. For instance, in the world of technology, the decision to choose from many alternatives is reached after using a hierarchical decision. In addition, the decision cannot be made if experts are not involved.

\subsection{Scenario Planning}

The scenario planning approach is increasingly applied in many fields in the world of business. Today, the world is defined by uncertainty, innovation, and technological change. Fate is the inability to predict the future well. Scenario planning is frequently applied to explore uncertainty and complexity in many areas. Scenario means a representation of a particular future condition and the roadmap to move you forward to the future requirement. Scenario planning enables people to think strategically. People use scenario planning to forecast the future; forecasting brings about various versions of the possible futures that people need to achieve. Scenario planning is creative thinking concerning likely complexity and unknown futures [72]. Most organizations today apply scenario planning to improve the decision making process and to handle uncertainty by looking at many likely futures. The scenario makes it easier for organizations to focus on fate and enhance the importance of the whole process in decision making. 
After World War II, systematic utilization of scenarios to clarify the prediction of the future was initiated. It became essential when the United States department of defense applied them in military planning during the 1950s. During the 1960s, the United States used a scenario approach in fields such as healthcare forecasting, analyzing public policies, and in the process of making decisions. Predicted situations strongly influence decision making processes and the way people think; they can also spark public debates and discussions. Scenario planning should give a detailed outline of the likely futures, consider several options, focus all attention on the future, as well as try to correct the current perception and situation. Studies about the end create a different picture of the present. During the scenario building process, the participants need to be encouraged by considering various options other than the already existing ones in the organization. Encouragement helps to explore new possibilities as well as beautiful ideas [72].

Earlier research shows that scenario planning methods, uncertainty, and unpredictability as well as instability of the general environment of the business are closely correlated. Increased uncertainty has promoted the need to identify future trends as well as the desired business condition. So, there is increased use of scenario because of the enhanced unknown and a detailed state of the operating field. Generally, situations can be short term or long term, but they are more useful when they are long lasting. Long term planning and strategic prediction make it possible to adapt quickly to changes as they occur. Future uncertainty becomes more complex as we move toward the desired future. 
Scenario building is broadly applied at the national as well as the corporate level. Shell Oil Company was among the pioneer companies to use a scenario approach, and it worked well. The process assisted the company in overcoming the unexpected economic difficulty, which saw prices of oil escalate too high during the 1970s. Situations make organizations more flexible and innovative and help them in preparing for the likely unexpected occurrences. Earlier research points out that, following the first oil shock, companies applying the scenario planning approach increased their returns by doubling them. It was evident that most of the large companies used a scenario planning approach.

Scenario building strategy is based on three major principles: identifying the future elements, changing perception so that we can see the real picture of the situation. The scenario opens up several promising future conditions to expand the way of thinking of the involved participants. Therefore, scenario planning forms many bright futures as shown in Table 5 below [73].

\begin{tabular}{|c|c|c|}
\cline { 2 - 3 } & \multicolumn{1}{|c|}{ Projection } & Scenario \\
\hline Characteristics & $\begin{array}{c}\text { Trying to make the precise prediction of } \\
\text { the occurrences, which are oriented to the } \\
\text { past }\end{array}$ & It is focused on the future \\
\hline Basis & It is about probabilities & Based on plausible factors \\
\hline Temporal scope & $\begin{array}{c}\text { It is mainly short-term or medium-term } \\
\text { planning }\end{array}$ & $\begin{array}{c}\text { Mainly medium-term or long- } \\
\text { term planning }\end{array}$ \\
\hline Decision making & Enthusiasm & $\begin{array}{c}\text { Elective situations structure the } \\
\text { reason for the change }\end{array}$ \\
\hline factors & realities, quantitative, goals, certainties & $\begin{array}{c}\text { It is object-oriented and } \\
\text { emotional, individual and } \\
\text { uncertain and subjective }\end{array}$ \\
\hline
\end{tabular}

Table 5: The difference between projection and scenario (SOURCE: Liu, Li \& Ding, 2020). 
As seen from the literature about scenario planning, scenarios have different meanings for different people and situations. Systems are created for other uses. Based on perception, plans may be classified as descriptive and normative. Illustrative scenarios are objectively coordinated and react to arrangement arranging worries to accomplish the set goals. Situations are likewise characterized based on the situation point (problem-explicit versus global system), the expansiveness of the situation scope (one sector versus multi-sector plots), the focal point of activity (environmental versus policy scenarios), and the aggregation level (micro versus macro scenarios).

\subsubsection{Scenario Planning as a Tool for Strategic Foresight}

The scenario planning approach is the highly preferred tool for every futureoriented project. In general, the term foresight means long term future-oriented events. Therefore, strategic vision means "the capacity to make and keep up a high-caliber, sound, and practical forward view, and to utilize the experiences emerging in helpful, authoritative means." Technology foreknowledge is a procedure to distinguish the vital research fields prone to yield the best financial and social advantages. It uses strategies to investigate the predicted futures in science; in terms of innovation and invention, economic progress, and social success. In an expected venture, the scenario planning assists in preparing well for the future and enhancing the view of chances and choices [74].

Scenario planning may fundamentally improve the convenience of a critical premonition venture and praise the prescience procedure. Scenario improvement exercises are the core of every future-oriented study. An examination of 860 future 
investigations uncovered that scenario planning is among the most frequently utilized systems in these future studies. In vital foresight ventures, scenarios are used to achieve a broad scope of targets. Significant parts of foresight are to investigate the extent of potential prospects and different essential alternatives. Situation examination assists with investigating these elective prospects and deliberately detailing and break down various vital options. Additionally, investigating future vulnerability is a critical piece of a foresight venture which can be practiced through scenarios [74].

The future vulnerability can be assessed by considering numerous alternatives over various situations, each delineating a brief look at the likely future. In future examinations, conditions feature ramifications of conceivable future framework cease and their suggestions. Ringland describes two significant functions of scenarios in supporting foresight researches; the first is giving a surely knew technique to investigate the future, and the second is introducing a lot of mental models. Cairns et al. express that situation strategies offer an empowering component to advance prescience exercises over different organizations. Along these lines, it tends to be inferred that scenario planning is a profoundly standard procedure which is broadly utilized in strategic foresight studies (Villa \& Cozzani, 2016).

\subsubsection{Strengths and weaknesses of scenarios and scenario analysis}

From the discussed literature on scenario planning, we can come up with several benefits. The strengths include the following: 
1) Scenarios predict the future through innovations and positive thinking about probable future trends as we move towards achieving the set goals. In other words, it creates a platform for people to think better on how to achieve the desired future. A scenario is a tool that makes the whole learning process more manageable.

2) Scenario planning embraces uncertainty, unpredictability, and vagueness of the future; as a result, it sets the foundation to kick off the journey towards the end.

3) Scenarios assists to monitor and identify potential challenges that are likely to occur in the future from the implementation process.

4) As the scenario planning process stimulates creativity and significant debates, it should be able to minimize and avoid any possible biases and errors that might occur in the process.

5) On many occasions, many companies find themselves in a situation where they test numerous hypotheses, predicting different futures and all the necessary in every underlying driving force [75].

Weaknesses of scenario planning include the following:

1) The achievement of the situation investigation is dependent upon numerous conditions. Circumstances require a constant update, refinement, and control by a particular group.

2) The interface of situations and chiefs may be disregarded or ignored. Conditions are inadequate if not incorporated into the dynamic procedure. 
3) Situation arranging is tormented with dubiousness, uncertainty, and powerlessness to different translations or concretizations, and offers, best case scenario some direction as it were. As a rule, instruments, including situation arranging and probabilistic hazard models, are just equivalent to the individuals that create or use them.

4) Insufficient for transient arranging: The consistent redundancy of the activity may primarily include disarray, as the approach doesn't have any significant bearing great to specialized choices that must be actualized for the time being. Situations require taught observing, examination, and correspondence process.

5) Intellectual inclinations: For instance, a plan that incorporates 3 situations depicting elective results along a solitary measurement is dangerous because numerous supervisors can't avoid the compulsion to detect the center situation as a gauge. A plan dependent on 2 circumstances raises a comparable hazard if one is effectively observed as romantic and the other skeptical, among others.

6) Situations can prompt "a feeling of lack of concern, of having every one of your wagers secured." Scenarios can't occupy the full scope of (future) conceivable outcomes; they leave you uncovered when situations give the most solace. We usually are excessively idealistic going into a downturn and overly critical as we move out. Nobody is insusceptible to this snare, including proficient developers of situations and the organizations that utilization them [75]. 


\subsubsection{Quantitative Scenario Development Methods}

There are many philosophies for creating situations with numerous typical attributes. Because of an enormous number of scenario advancement strategies and models as introduced in this paper earlier, a few writers depict it as a "methodological tumult." The procedures below are considered the most mainstream and broadly utilized quantitative methods for building situations. These strategies are viewed as cutting edge for building up the quantitative conditions [76].

1. Interactive Cross Impact Simulation (INTERAX);

2. Interactive Future Simulations (IFS);

3. Trend Impact Analysis (TIA).

\subsubsection{Limitations of Quantitative Scenario Methods}

The scenario building techniques only rely on already existing information; as a result of this, it may not produce accurate results. Unlike qualitative techniques, which are mainly long term, quantitative approaches are only useful in short term projects. The validity of quantitative methods diminishes as time passes; this is the case because the current trends change with time. Alternately, the usefulness of qualitative methods increases as time passes. Some earlier studies argue that, sometimes, both quantitative and qualitative researches complement each other, and they may be used together [75]. 


\subsubsection{Scenario planning in healthcare}

Rather than expecting that difficulties will strike, hospital management, specialists, and supervisors should act ahead of time to upgrade and establish sustainable health. This section features the significance of scenario planning in health centers. It proposes an expounded meaning of the hospital stakeholders and characterizes the impact components to which emergency clinics are presented. The reason for this paper is to offer another hypothesis for applying situation-based forecasts of occasions in the vital arrangement of medicinal services tasks to more readily oversee social insurance expenses and assets and increment the proficiency of usage. The hypothesis presents a theoretical model that predicts the occurrence of occasions in anticipation of ailments as indicated by various potential situations, given various affecting factors [73].

Scenario planning offers an integral way to deal with traditional preparation. Traditional strategic planning comprises of foreseeing the future at a solitary point on a picked time horizon and drawing the popular designs to address such a lot. Then again, scenario planning makes tales about numerous presumable potential fates on a given time horizon and maps the favored models to address the various portrayed potential prospects. It recognizes expressive powers influencing social insurance conveyance frameworks, tests presumptions for the future, evaluates elective fates, and can shape an establishment for future planning. Scenarios are especially helpful in a quickly changing world with moving extreme conditions, for example, social insurance frameworks [73]. 


\subsubsection{Factors affecting scenario planning and analysis}

The model depends on the segments of situation arranging, which partitions our insight into two expansive areas certainties and uncertainties, at a given time. The principal part, patterns, and convictions cast the past forward, perceiving that our reality has significant force and congruity, for example, making presumptions about the visualization of an ailment dependent on past recorded information and patterns. The next part, evident vulnerabilities, includes the indeterminable, such as future achievement rates and results of ailment guess. The craft of situation arranging lies in mixing the known and the obscure into a set number of inside steady perspectives on the future that lengthen a broad scope of conceivable outcomes [73].

\subsection{Research Design and Methodology}

A quantitative research method is used to address the research objectives. An initial research model is developed, which contains five main barriers and sixteen sub barriers. The research aim is to conduct an empirical research approach based on theoretical models and collect data obtained from the telemedicine experts and users.

\subsubsection{Data Collection}

In this research, the link to the initial hierarchical decision model (HDM) was sent by email to the experts and people who use the telemedicine system to collect the data. The targeted research audience would be the experts in telemedicine system, physicians, nurses, clinical staff and patients. Data collection was carried 
out through expert panels. Table 6 below summarizes the data sources for the HDM model.

\begin{tabular}{|c|c|c|c|c|c|c|c|}
\hline & $\begin{array}{c}\text { Financial } \\
\text { barriers }\end{array}$ & $\begin{array}{c}\text { Technical } \\
\text { barriers }\end{array}$ & $\begin{array}{c}\text { Logistical } \\
\text { barriers }\end{array}$ & $\begin{array}{c}\text { Ethical } \\
\text { barriers }\end{array}$ & $\begin{array}{c}\text { Cultural } \\
\text { barriers }\end{array}$ & $\begin{array}{c}\text { Perceived } \\
\text { Usefulness }\end{array}$ & $\begin{array}{c}\text { Perceived } \\
\text { Ease of Use }\end{array}$ \\
\hline $\begin{array}{c}\text { Hospital } \\
\text { Administration }\end{array}$ & $\sqrt{ }$ & $\sqrt{ }$ & $\sqrt{ }$ & $\sqrt{ }$ & & $\sqrt{ }$ & $\sqrt{ }$ \\
\hline Clinical Staff & & $\sqrt{ }$ & $\sqrt{ }$ & $\sqrt{ }$ & $\sqrt{ }$ & $\sqrt{ }$ & $\sqrt{ }$ \\
\hline Physicians & $\sqrt{ }$ & $\sqrt{ }$ & $\sqrt{ }$ & $\sqrt{ }$ & $\sqrt{ }$ & $\sqrt{ }$ & $\sqrt{ }$ \\
\hline Nurses & & $\sqrt{ }$ & $\sqrt{ }$ & $\sqrt{ }$ & $\sqrt{ }$ & $\sqrt{ }$ \\
\hline Patients & $\sqrt{ }$ & $\sqrt{ }$ & $\sqrt{ }$ & $\sqrt{ }$ & $\sqrt{ }$ & $\sqrt{ }$ \\
\hline IT Staff & $\sqrt{ }$ & $\sqrt{ }$ & $\sqrt{ }$ & $\sqrt{ }$ & & $\sqrt{ }$ \\
\hline
\end{tabular}

Table 6 : Data sources.

\subsubsection{Expert Panel}

An expert is a person who has the background and knowledge in subject area and is considered qualified to answer those questions [77]. In other words, an expert is usually someone who has an extended or intense experience through practice and education in a particular field [78]. However, an expert is not always necessarily someone who has professional or academic qualifications. Experience in a particular field can qualify somebody to be recognized as an expert [79]. An expert panel is a group of participants who have expertise in a particular area. Expert panels are formed to validate the elements in Analytic Delphi research and to quantify the relationships among the decision elements at all levels of the decision hierarchy in the HDM.

Each expert panel is required to have a balanced representation of opinions. The members of an expert panel should reflect current knowledge and perception as well as be impartial to the research findings [80]. It is critical that the right balances of experts are employed to ensure the accuracy and reliability for 
the best results. The selection of panel experts is imperative for the success of Analytic Delphi research. The judgmental data provided by experts is directly based upon their knowledge, capability, and experience, and therefore strongly influence the final research outcome [81]. Consequently, the expert panel criteria are vital.

The experts have several roles during the research process such as to validate and construct the content of the hierarchical model, to provide the judgmental data of relative impacts, and to validate the results of research [82]. The roles of the experts in this research are to identify critical adoption barriers, provide relative weights for adoption barriers, and help identify other experts and validate the model and results. In general, expert panels review, finalize and validate the HDM and the associated perspectives, criteria, and factors.

\subsubsection{Experts Selection Criteria}

Usually experts with different backgrounds are brought in for the expert panels. It is key for the team of experts to be considerably diverse so the problem under consideration is thoroughly analyzed from multiple viewpoints [77]. The diversity in an expert panel leads to better performance because it gives considerations from numerous perspectives and a wider range of alternatives [83]. There are several criteria for selecting expert panel members and there are some general criteria for expert panel selection such as [60, 84-88]: 


\subsubsection{Expertise in the topic}

It is necessary that the knowledge of the expert be matched with the research area, a selection that has to be done carefully. The expert panel members should have an in-depth knowledge in the area or field under consideration. The experts should be at the top of their fields of technical or scientific knowledge and have the qualifications appropriate to the question under investigation. If an expert is from an industry, the level of expertise is determined by his/her position. If the expert is from academia, he/she must have several publications related to the subject. The number of publications and cited works helps to identify the level of expertise.

\subsubsection{Availability and willingness to participate}

It is very important to have experts who are available during the study timeline, willing to participate, and committed throughout the complete research process.

\subsubsection{Balanced background}

The expert panel should be well balanced, meaning that the members come from different backgrounds such as academia, industry, and government.

\subsubsection{Balanced biases}

It is likely individual members in the panel are motivated toward certain elements in the model, so they may give more favorable judgments to those elements. The experts should come from different departments within an 
organization in order to balance perspectives and biases. This bias may stem from their work experience or personal interpretation of the question.

\subsubsection{Balanced viewpoints}

An expert should possess an ability to cross over traditional viewpoints and look at the problems, not only from a known and safe point of view, but also from unconventional angles. Similar to individual biases, different viewpoints of the experts may influence their comparison results. The different viewpoints of the experts in a panel need to be offset. The experts have to be interested and open to a wide range of knowledge and not limited to their own field.

\subsubsection{Avoiding domination by loudness}

Opinions vary during the data collection from experts. It is common to see some experts speak louder than others to express their opinions, which can influence the results. In a meeting session, it is important to prevent any individual member to impose his or her own views and judgments on others. Disagreements should be addressed, and even minor ideas should not be ignored. In my study, data were collected via emails and web quantification instruments and not face-toface or in-group meetings.

\subsubsection{Avoid silent bystanders}

Some members in a group meeting may avoid giving their opinions just to alleviate the conflicts among the group members. This is a common situation that occurs in data collection when experts are not expressing their opinions because of shyness. It is necessary to solicit all experts' personal ideas, so their inputs 
contribute to the improvement of the results. A silent bystander chooses to be silent and refuses to be vocal in order to avoid conflict with other experts. Some experts who are silent bystanders might have great opinions, but those opinions could be lost. In my study data were collected via emails and web quantification instruments and not face-to-face or in-group meetings.

\subsubsection{Expert Selection Methods}

There are a number of methods to find the experts for research. The most common approaches used to make a list of panel members are described below [89-93]:

\subsubsection{Personal Connections}

Personal connections are a convenient and common way to create the list of experts. The researcher invites his/her connections that are believed to have sufficient knowledge of the subject to participate in the expert panel. The advantage of the method is time and effort efficiency, but the disadvantage is the experts might not be representative of the field.

\subsubsection{Snowball Sampling}

Snowball sampling is a common expert identification where known experts recommend potential experts from among their acquaintances or networks. A researcher begins with a few known experts, asks for more names from them, and repeats until he or she has more names than are actually needed. Thus, as more experts are recruited, the group grows like a snowball until enough experts are identified. This method is common among the researcher networks where one 
researcher often knows of other prominent figures in the field where there is no easy direct access.

\subsubsection{Academic Sources}

Some academic websites have a searchable database of professors claiming expertise. A background of a professor can be verified from the professors' academic institution website, one can verify their background by perusing their $\mathrm{CV}$, papers published, and courses offered.

\subsubsection{Social Network Analysis}

Social network analysis refers to methods of analyzing social networks or structures. This method can be used in large databases where the researcher network can be considered a social network where one researcher usually cites others' papers in his or her paper. Personal profiling and document profiling are the two common approaches for this method. The search keywords describe the person in personal profiling, and in document profiling keywords are used for document searches.

\subsubsection{Citation Analysis}

Using citation databases to determine expertise based on papers published and referenced is a good method to identify experts. Associated reporting and analysis also enable grouping the authors into specialty areas. 


\subsection{Research Validity}

The panels of experts from both academia and healthcare were validating the model. Validation by definition is a test of whether the model is an adequate (sufficient) representation of the elements and their relationships of the actual systems it's referring to with their underlying importance to the model's planned experiments. The validity of the initial research was tested by three measures applied at different phases of the research. These measures are content validity, construct validity, and criteria-related validity.

\subsubsection{Content validity}

Content validity refers to the degree to which a measure represents a given domain of interest. Content validity is going to be tested during the model development phase. An initial assessment model has been developed based on the literature. Then, based on experts' evaluation, unnecessary variables will be eliminated, or new variables will be added to the model.

\subsubsection{Construct validity}

Construct validity tests the readiness of the instruments to gather data from respondents. Construct validity is going to be ensured after the model development phase. Criteria-related validity refers to the degree of effectiveness of a model in predicting real life phenomenon. Criteria-related validity is going to be ensured after the analyses are complete. Table 7 below summarizes the three types of validity for the initial research. 


\begin{tabular}{|l|c|c|c|}
\hline \multicolumn{1}{|c|}{ Validity } & Description & Method & When \\
\hline $\begin{array}{l}\text { Content } \\
\text { validity }\end{array}$ & $\begin{array}{c}\text { Degree to which a measure } \\
\text { represents a given domain of } \\
\text { interest }\end{array}$ & $\begin{array}{c}\text { Expert evaluation, and } \\
\text { literature review }\end{array}$ & $\begin{array}{c}\text { During the model } \\
\text { development }\end{array}$ \\
\hline $\begin{array}{l}\text { Construct } \\
\text { validity }\end{array}$ & $\begin{array}{c}\text { Degree to which a initial research } \\
\text { approach complies with its } \\
\text { underlying theories }\end{array}$ & Expert evaluation & After the model development \\
\hline $\begin{array}{l}\text { Criteria- } \\
\text { related } \\
\text { validity }\end{array}$ & $\begin{array}{c}\text { Degree of effectiveness of a } \\
\text { model in predicting real life } \\
\text { phenomenon. }\end{array}$ & $\begin{array}{c}\text { Expert evaluation (experts } \\
\text { who have not provided } \\
\text { judgment) }\end{array}$ & After the analyses \\
\hline
\end{tabular}

Table 7: Validity of the initial research approach.

\subsection{Data Analysis}

After getting the data from experts and system users, the results will be validated, and related analysis will be conducted.

\subsubsection{Inconsistency}

Inconsistency is related to an individual expert's response to the judgment quantification instrument. Consistency is the degree to which an individual is consistent in his/her own judgment. Inconsistency describes a situation where the expert judgment changes over time. As judgment quantification relies on the knowledge of experts, putatively, data from human subjects may be inconsistent at times. Inconsistency is measured as the variance in the values of each orientation relative to the mean [94].

Inconsistency values for the constant sum method are calculated as follows [60]: For $n$ elements, the constant sum calculations will result in a total of $n$ ! orientations with vector values represented by $r_{1}, r_{2} \ldots r_{n}$ for each. If the expert is totally consistent, the relative values will be the same for each orientation. 
Otherwise, if inconsistency exists it will result in differences in the relative values in different orientations. According to Kocaoglu's research, if the inconsistency level is less than $10 \%$ or 0.1 , the related judgmental data should be acceptable [95].

$r_{i l}=$ relative value of the $\mathrm{i}^{\text {th }}$ element in the $\mathrm{j}^{\text {th }}$ orientation for an expert.

$\bar{r} i=$ mean relative value of the $\mathrm{i}^{\text {th }}$ element for that expert:

$\bar{r} i=\left(1 / n_{i}\right) \sum_{j=1}^{n i} r i j$

Variance in the relative value of the $\mathrm{i}^{\text {th }}$ element:

$\left(1 / n_{i}\right) \sum_{j=1}^{n i}(r i-r i j) 2 \quad \mathrm{i}=1,2, \ldots \mathrm{n}$

Inconsistency of the expert in providing relative values for the $n$ elements is defined as:

$$
\text { Inconsistency }=\sqrt{(1 / n) \sum_{i=1}^{n}\left(\frac{1}{n i}\right) \sum_{j=1}^{n i}\left(r_{i}-r_{i j}\right) 2}
$$

If the inconsistency value is above $10 \%$ ot 0.1 , the input data need to be verified with the original expert by explaining the inconsistency measurement, and having the expert improve his/her judgmental inputs.

\subsubsection{Disagreement}

Disagreement is the extent to which the participants of an expert panel are in difference to each other in their judgments. It is natural to have different opinions among the experts during the research process. The diverse social backgrounds and working experiences of the experts may cause significant differences in 
opinions or perspectives toward any research topic. Even if all the experts have same expertise and knowledge, they may think in different ways to approach the same problem and come up with different judgment. Moreover, usually the expert judgment is obtained in an uncertain situation, where no clear standards or welldeveloped theories exist $[96,97]$. Therefore, the expert judgment may lead to some disagreement among the experts. However, differences among the experts brings different perspectives and research indicates it brings a better chance of covering the right solution $[77,98]$.

\subsubsection{Sensitivity Analysis}

The data sensitivity analysis for the hierarchical decision model (HDM) was based on Chen's dissertation and Kocaoglu's research $[99,100]$. Chen and Kocaoglu introduced the sensitivity analysis algorithm for HDM, which can be used to calculate the allowable ranges, tolerance, operating point sensitivity coefficient (OPSC) and the total sensitivity coefficient (TSC). In this method, tolerance is defined as the allowable range in which a contribution value can vary without changing the ranking order of the bottom level alternatives. With this method, algorithms were developed based on a series of mathematical deductions. It is an accurate and comprehensive method to examine the impact of changes in different levels of HDM on the ranking of the alternatives. 


\subsection{Research Framework}

1. Literature Review.

1.1. Review the literature on teleconsultation adoption.

1.2. Identify the Research Gaps.

1.3. Identify the Research Objectives.

1.4. Identify the Alternative Solution.

1.5. Develop Research Questions.

2. Research Model Development.

2.1. An initial HDM model is developed, based on the literature review, 3. Data collection.

3.1. Identify the research audience.

4. Data Analysis.

4.1. Analyze the data by using appropriate methods.

5. Results, discussions and conclusion. 


\section{CHAPTER 4: CASE STUDY MODEL DEVELOPMENT}

The literature highlights researchers from different fields and with several interests who recognize the important role of telemedicine in the healthcare industry. There are many researchers conducting studies in order to recognize the barriers that affect the adoption and use of telemedicine systems. In this research I focused only on one type of telemedicine application, which is the teleconsultation. There are several barriers impacting the adoption of telemedicine application through perceived usefulness and ease of use, which are the two important factors of the technology acceptance model (TAM).

\subsection{Adoption Barriers}

Teleconsultation holds great promise for new, cost effective, high quality healthcare services, as well as efficient methods of delivering healthcare across geographic distances. However, these benefits are constrained by five main barriers: financial, technical, logistical, ethical, and cultural.

\subsubsection{Financial Barriers}

Using limited financial and human resources in providing high-quality care efficiently for an increasing number of patients is a major challenge to many healthcare systems. Implementing a telemedicine program is constrained by many financial barriers such as the start-up and ongoing cost [101]. The financial barriers include everything related to the financial issue involved in implementation of telemedicine. 


\subsubsection{Start-up Cost}

The start-up cost includes initial fixed cost of the hardware, software, and technical assistance necessary to install the system, licensing fees, and the expense of maintaining the system [101]. There are many physicians who worry about the start-up cost of adopting telemedicine in their daily practices. In general, start-up cost is very high in the healthcare industry and for that, high start-up costs have presented a barrier to many healthcare facilities.

\subsubsection{Ongoing Cost}

The ongoing cost includes all the financial expenditures after implementing telemedicine in order to work effectively and efficiently [102]. The ongoing cost includes updating the telemedicine system, new licensing or renewals, and new devices.

\subsubsection{Technical Barriers}

Adoption of any telemedicine system has many technical barriers such as computer skills, training, technical support, and infrastructure. The technical barriers include everything that is related to the technical issue involved in the implementation of telemedicine.

\subsubsection{Computer Skills}

Sufficient computer skills are required for applying telemedicine [103]. The descriptor 'sufficient computer skills' is defined as being comfortable working within the healthcare environment and with healthcare technology in order to enable all staff members to participate in the telemedicine network. It depends on the 
reliability and professional use of technology to ensure the delivery of a reliable telemedicine service. Some of the health professionals will work with modern information and communication technologies like computers, printers, the Internet, and digital cameras for the first time. Therefore, the following activities are needed to apply telemedicine:

- Use a digital camera to acquire good quality images (Xx-ray images and ultrasound images).

- Use the computer to write and edit plain text, and to store images taken with the digital camera

\subsubsection{Training}

Training is needed for using the system effectively and efficiently [104]. The computer training needs to be done in computer classes with the respective technical infrastructure in order to be as practical as possible and to provide the users with real hands-on training. As users need much more attention in this type of courses, class sizes should not be greater than 4 to 6 persons.

\subsubsection{Technical Support}

Technical support is needed after implementing the system [104]. Technical support staff members can help answer questions about telemedicine programs. To help with efficiency, technical support staff may be shared across collaborating organizations. Ongoing, accessible technical support for telemedicine systems is key to ensuring their sustainability. Technical support must be available 24 hours daily to ensure patient safety. 
Internal IT departments often provides such technical support. Also, vendors can provide technical support during and after implementation. The level of support available to projects from vendors varied, for example many small companies were closed during weekends and evenings. When vendor support was not available 24 hours a day, 7 days a week, projects reported greater potential for negative impact on patient safety and mission-critical patient services.

\subsubsection{Infrastructure}

The technical infrastructure requirements are largely dependent on the targeted services associated with the project. However, nearly all telemedicine programs depend on high rates of data transmission, so access to broadband Internet is necessary. Specialized application software, data storage devices, database management software, and medical devices capable of electronic data collection, storage and transmission are all key components of the Telemedicine infrastructure [105]. There is a need to provide infrastructure that does not have an upper limit on quality of service and allows telemedicine to address mobility, usability, interoperability, intelligence, and adaptability in a systematic way. However, even if the telemedicine infrastructure is strong, problems can still occur.

\subsubsection{Logistical Barriers}

There are several logistical barriers limiting the widespread use of telemedicine systems such as licensure, credentialing, malpractice, and reimbursement. The logistical barriers include all the regulation issues involved in implementation of telemedicine. 


\subsubsection{Licensure}

Licensure authority defines who has the legal responsibility to grant a health professional the permission to practice their profession. Licensure is legal documents to practice the art and science of medicine [106]. Licensure portability is an often-debated topic. "Where should a healthcare provider be licensed"? "Which states allow healthcare providers to consult with one another across state lines"?

The purpose of licensing healthcare professionals is to protect the public from incompetent or impaired practitioners. Practicing medicine requires a certificate of licensure from the state in which the practitioner is working and may require licensure in the state where the patient is located. As the use of telecommunication to complement healthcare service delivery becomes readily available, some states have responded with policies that accommodate patient choice, peer consultation, and health provider shortages [107].

A practitioner must be licensed, or follow state reciprocity rules, prior to working in a state. In light of telemedicine, licensure requirements can be complicated. If all patient interactions are within the state in which the physician is licensed, then he/she maintains licensure in good standing. But if a physician electronically interacts with patients in other states, he/she must be licensed or register in each state in which he/she electronically practices. Practicing medicine without a license in the state in which you are electronically practicing may incur civil and/or criminal penalties [107]. 


\subsubsection{Credentialing}

Credentialing is a process of objectively evaluating and verifying a practitioner's current licensure, training, experience, competence, and ability to provide particular services or perform particular procedures. Credentialing is the rules that allow hospitals to use telemedicine services for their patients [108]. It is usually carried out by the organization that will bill for the provider's services. Whether or not the provider must be credentialed depends on the type of facility but is not normally related to whether or not the provider is participating in telemedicine applications.

The credentialing requirements promulgated by the Joint Commission and CMS do not have the same requirements.

\subsubsection{Malpractice}

Medical malpractice is a deviation from the accepted standards of practice in the medical community and causes injury or death to a patient for whom the physician has a duty of care. Telemedicine malpractice is the medical professional liability of practicing telemedicine [109]. State law generally governs medical liability. Standards and regulations for medical malpractice vary by state. Medical professionals are required to maintain professional liability insurance to offset the risk and costs of lawsuits based on medical malpractice.

For telemedicine encounters involving direct care of patients, most medical malpractice insurance covers only face-to-face encounters within the state in which the doctor practices and is licensed. Consequently, doctors who provide telemedicine services to patients outside of the State in which they are licensed 
can be exposed to uninsured claims if state law requires the physician to be licensed in the state where the test results are delivered.

\subsubsection{Reimbursement}

Financial incentives for providers using the system may cause telemedicine constraints by the healthcare reimbursement processes as both public and private insurance may not pay for telemedicine services or may pay only for some services under limited circumstances [110]. In a recent survey done by "Foley" in 2014, most of the experts mentioned that they have difficulties seeking and receiving reimbursement and that is the one and most important barrier for implementation of telemedicine. Approximately $60 \%$ of the experts reported that they either didn't receive any reimbursement for a telemedicine visit $(41 \%)$ or received lower rates from managed care companies for telemedicine than in-person care $(20 \%)$ as shown in Figure 14.

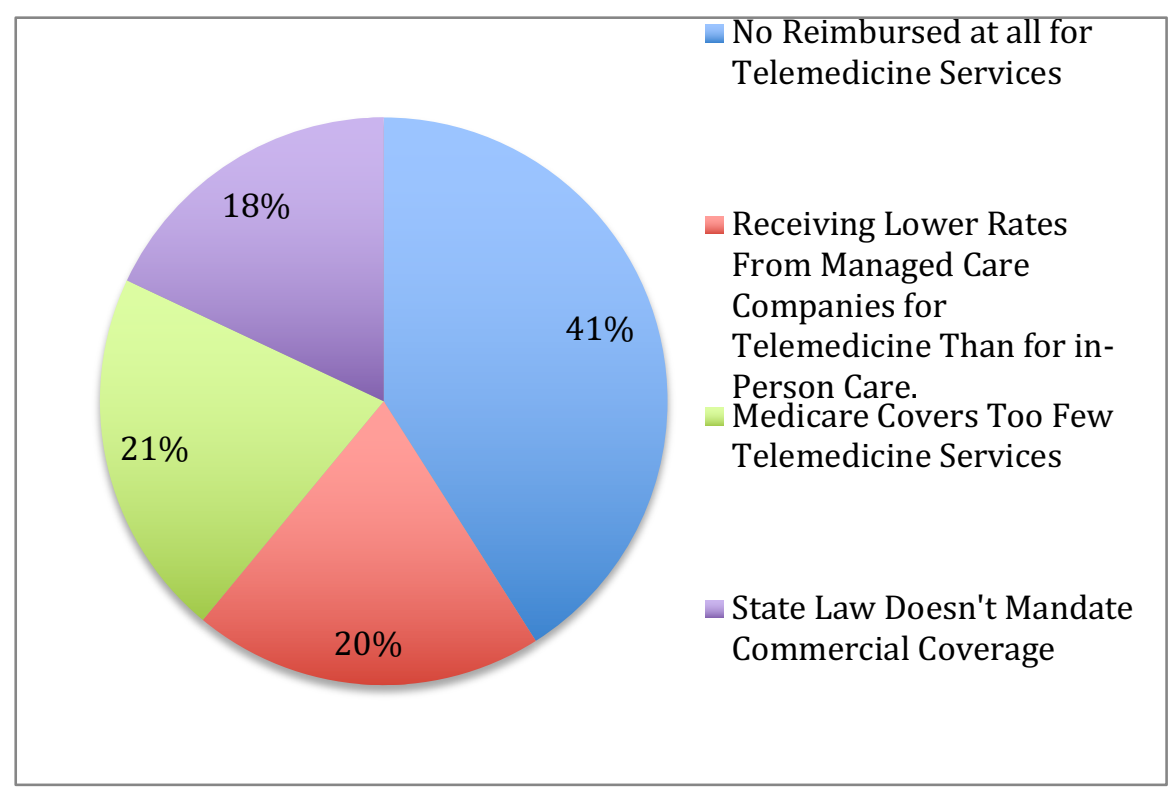

Figure 14 : The most significant concern regarding reimbursement of telemedince services. (Source: Foley survey - 2014). 


\subsubsection{Ethical Barriers}

The adoption of any telemedicine practices questions the system from an ethical point of view regarding whether the practice of telemedicine increases or decreases the level of patient care. The main issue should focus on the impact telemedicine has on the patient/physician relationship and whether telemedicine increases the quantity and quality of patient care. Ethical issues that rise in the adoption of telemedicine are privacy and security, beneficence, and justice.

\subsubsection{Privacy \& Security}

The privacy and security for all personal health information and medical data is mandated at the federal level by the 1996 Health Insurance Portability and Accountability Act (HIPAA) [111].

\subsubsection{Beneficence}

Beneficence involves the obligation to prevent and remove harms and to promote the good of the person by minimizing possible harms and maximizing possible benefits [112].

\subsubsection{Justice}

Justice recognizes each person should be treated fairly, equitably, and be given his or her due. Justice dictates that patients should be treated in a similar manner if at all possible [112].

\subsubsection{Cultural Barriers}

Cultural barriers limiting the use of telemedicine application include physician acceptance and patient satisfaction. 


\subsubsection{Physician Acceptance}

The teleconsultation model depends largely on acceptance by healthcare providers (physicians, nurse practitioners, and physician assistants) [113]. We should recognize that user acceptance is important, but physician acceptance is critical [114]. Physicians are the most important users of telemedicine technology and their acceptance and satisfaction will play a crucial role in further acceptance of telemedicine [115]. Physician acceptance includes everything related to their discomfort in the use of the new technology equipment in their daily practices and in treating patients at a distance.[116]

\subsubsection{Patient Acceptance}

Telemedicine can have a positive impact on patient behavior towards managing his/her illness when it is feasibly reliable and the user is satisfied with the system [117]. Patients' acceptance of teleconsultation services include anything that can decrease their acceptance of using the system [116].

\subsubsection{Patient Satisfaction}

"Satisfaction is an accepted indicator of the performance of a health-care service. It reflects patients' values and expectations regarding various aspects of a health service" [117]. Patients are satisfied when there is a match between the care that is expected with what is received. Patient satisfaction includes anything that could decrease their satisfaction with using telemedicine [118]. 


\subsection{Alternative Solutions}

There are several alternative solutions to address adoption barriers. Some of the alternative solutions related to the regulations and standardized processes in implementing the teleconsultation system would portray government and individuals playing the main role towards successful adoption. After reviewing the literature, I identified eight possible solutions as following:

\subsubsection{Government Support}

Includes all governmental support and helps to adopt the system. The government support can take place in several types such as providing some grants for telemedicine projects and providing technical assistance and developing some policies encouraging the use of telemedicine applications [172]. Also evaluating the use of existing telemedicine technologies and programs and providing some recommendations to enhance the adoption.

\subsubsection{Continual Training}

Continual training includes all types of training and assistance to use the system effectively and efficiently. All technology requires updating training, installing videoconferencing equipment in a hospital or health center, along with sufficient training needed to encourage and maintain use for telemedicine. Telemedicine staff must be properly trained not just in how to turn on the equipment, but also in how to implement it effectively for consultations, education and administrative purposes [173]. Trainings need to be continual, practical and in 
different levels with clear straightforward protocols and user-friendly instruction manuals.

\subsubsection{Develop Guidelines for the Use of Telemedicine}

As the number of physicians using telemedicine to diagnose and treat patients is growing, it is essential to develop guidelines and regulations to provide states a framework to use when creating their own telemedicine laws. The guidelines should cover the evaluation and treatment of the patient, informed consent, continuity of care, referrals for emergency services, medical records, privacy and security of patient records, the exchange of information, disclosures, and functionality of online services making telemedicine technologies available and prescribing [174].

\subsubsection{Multi-State Licenses for Telemedicine}

Multi-state licensing would grant licenses for physicians to practice telemedicine across state borders. Physician practice is regulated in each state by a state medical board whose authority is granted by the state legislature. Each state board functions independently of other states leading to variations in policies from state to state; if practicing in multiple states, physicians will need to understand and comply with these restrictions [175]. Multi-state telemedicine licenses will address all of the various policies and make it easier for physicians to practice across state borders, allowing a physician to apply for the multi-state license through his or her state of principal licensure. 


\subsubsection{Multi-State Standards and Regulation}

Multi-state standards and regulation includes all standards and regulations necessary to adopt the system. The multi-state standards and regulations provide uniform quality of service to patients, and to promote reasonable and informed patient and provider expectations. The purpose of these standards and regulations is to assist physicians to provide effective and safe medical care for patient needs. These standards and regulations are fundamental requirements to be followed when providing medical and other healthcare services using telecommunications technologies, and any other electronic communications between patients, practitioners and other healthcare providers [175].

\subsubsection{Standard Processes for Reimbursement}

Obviously, reimbursement is very important in order to encourage use of telemedicine services. Standard processes provide mechanisms to reimburse providers and is used as a tool to ensure sustainability of the program. Federal and state policies on reimbursement could lead to more helpful policies for government and private payers [176]. Reimbursement standards help with billing concerns such as systematizing billing codes and reimbursement methods for telemedicine services.

\subsubsection{Ethical Codes}

Ethical codes include all medical and other professional codes used to adopt the system. There should be ethical and professional codes to obey in all aspects of telemedicine services. All healthcare providers must respect the ethical 
guidelines and codes when practicing telemedicine such as the confidential relationship between patient-physician and the quality of care [177].

\subsubsection{Education}

Includes all types of education that help to adopt the telemedicine system. Education is very important in healthcare service and can take place in several ways such as to educate patients on the use of the telemedicine programs and their advantages, by using such telemedicine programs [178]. Also, it could take place as a short course, seminar, or workshop, provided face-to-face or online.

\subsection{Adoption Theories}

In this section famous and important adoption theories are reviewed in some detail. Four important theories are described below:

1) The Theory of Reasoned Action (TRA),

2) The Innovation Diffusion Theory (IDT),

3) The Theory of Planned Behavior (TPB), and

4) The Technology Acceptance Model (TAM)

\subsubsection{The Theory of Reasoned Action (TRA)}

The theory of reasoned action (TRA) was developed in 1967 by Fishbein and Ajzen in order to predict behaviors and outcomes as shown in Figure 15. They assumed individuals are usually quite rational and make systematic use of information available to them. People consider the implications of their actual behaviors before they decide to engage or not engage in a given behavior" [119]. 


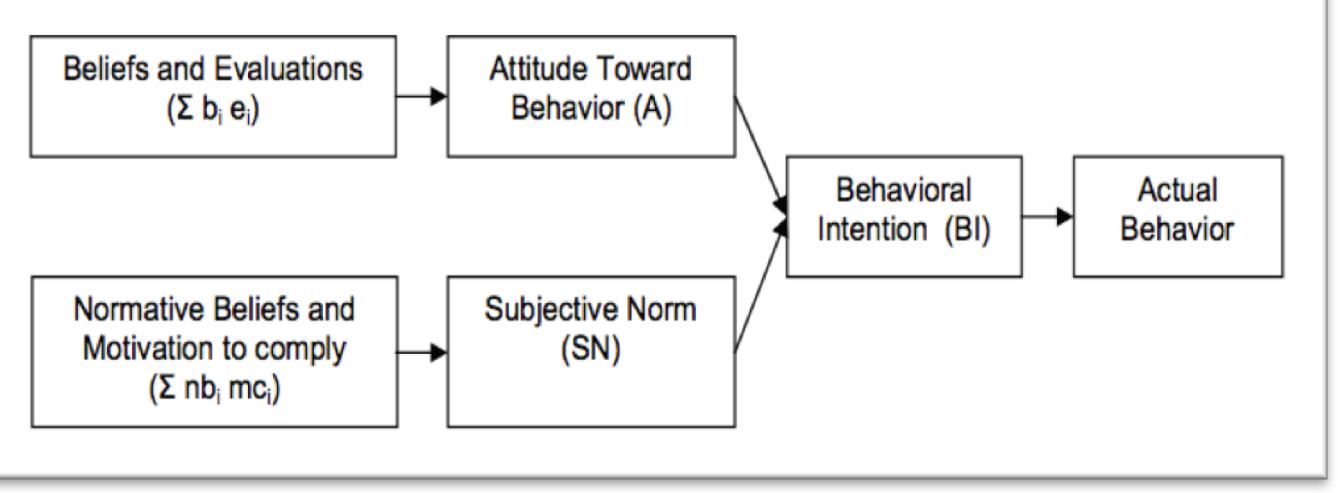

Figure 15 : Theory of Reasoned Action (TRA) ( Aizen and Fishben. 1980) [119].

Aizen and Fishben developed a theory that could predict and understand behavior and attitudes after studying all available behavior and attitudes studies. They introduced a new framework where the behavioral intention was the main predictor of actual behavior rather than the attitude. Ajzen and Fishbein revised and expanded the theory of reasoned action (TRA) in the early 1970s. The theory was used to study human behavior and develop appropriate interventions by 1980 [120] \& [121]. The social psychology models were the most studied by TRA concerning factors of consciously intended behaviors [122].

By applying TRA we can identify how and where to target strategies for changing actual behavior. We can also explain virtually any human action and predict and understand motivational influences on actual behavior that is not under the individual's volitional control. According to TRA, in order to define a person's performance of a specified behavior we have to determine his or her behavioral intention $(\mathrm{BI})$ that is jointly determined by the person's attitude toward behavior and subjective norm (SN) concerning the behavior in question. 


\subsubsection{Innovation Diffusion Theory (IDT)}

Rogers established the innovation diffusion theory (IDT) in 1962 which became a well-known theory for user adoption [123]. The end user is the core element in innovation diffusion. Innovation diffusion can be achieved through users accepting and using new ideas. The IDT is very helpful in explaining the innovation decision process. The theory can explain the elements of rate of adoption and the other categories of adopters. Also, it helps predict the probability and the rate of an innovation being adopted.

Tornatzky and Klein (1982) found that only relative advantage, compatibility and complexity, were consistently related to the rate of innovation adoption by applying meta-analysis of 75 diffusion articles [124]. In general, we can call diffusion a special type of communication, but only concerned with the spread of messages that are perceived as new ideas. The diffusion consists of 4 main elements that are the innovation, communication channels, time, and the social system.

\subsubsection{The Theory of Planned Behavior (TPB)}

Theory of reasoned action (TRA) has some limitations such as to when it's applied to the actual behaviors that are not fully under a person's volitional control. The theory of planned behavior (TPB) is just an extension of the theory of reasoned action (TRA) [125] . The central factor in the TPB is the individual's intention in performing a specific behavior as shown in Figure 16 [126]: 


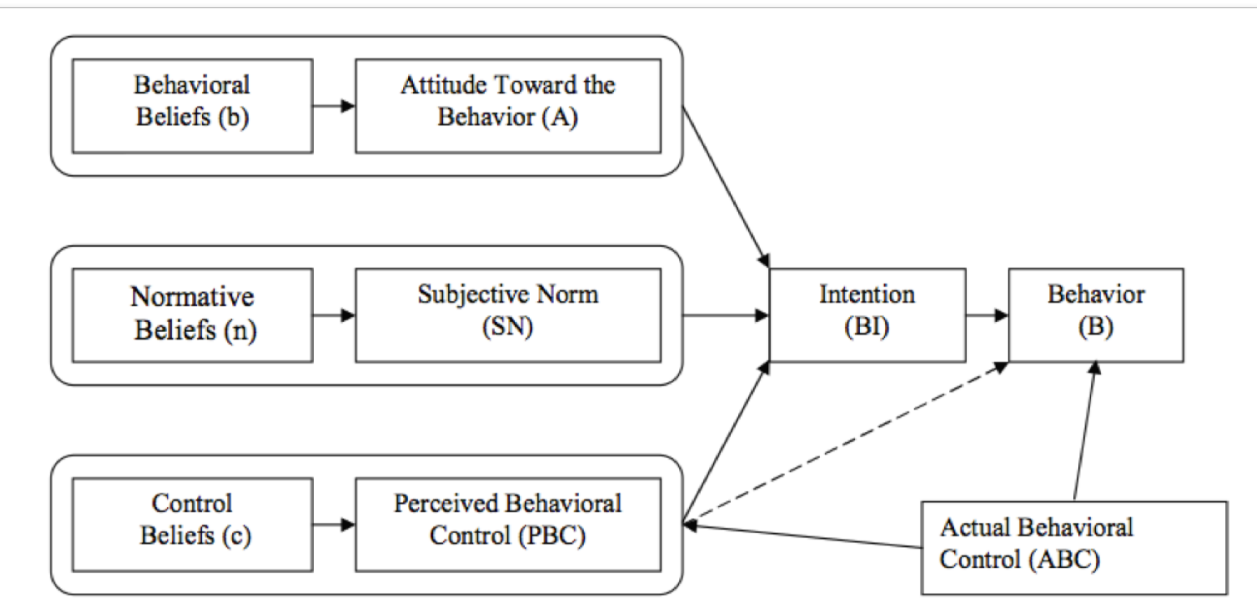

Figure 16 : Theory of Planned Behavior (TPB) (Ajzen, I. 1991) [126].

The motivational factors that influence actual behavior can be captured by intention such as how much of an effort people are planning to apply and how hard they are willing to try in order to achieve the actual behavior. In general, a person's performance is likely to equate to his/her intentions to engage in the actual behavior.

\subsubsection{Technology Acceptance Model (TAM)}

User acceptance of technology has been an important field of study for over two decades. Emerging information technology cannot deliver improved organizational effectiveness if it is not accepted and used by potential users. Fred Davis and Richard Bagozzi initial the technology acceptance model (TAM) [127] by developing the theory of reasoned action (TRA), which was formulated by Fishbein and Ajzen [128]. According to the model, the acceptance of a new technology by a user is based on two factors: perceived usefulness (refers to how much the user believes that the technology will help to improve the 
performance/efficiency) and perceived ease of use (refers to what extent the user is comfortable in using the features of the technology) as shown in Figure 17 below:

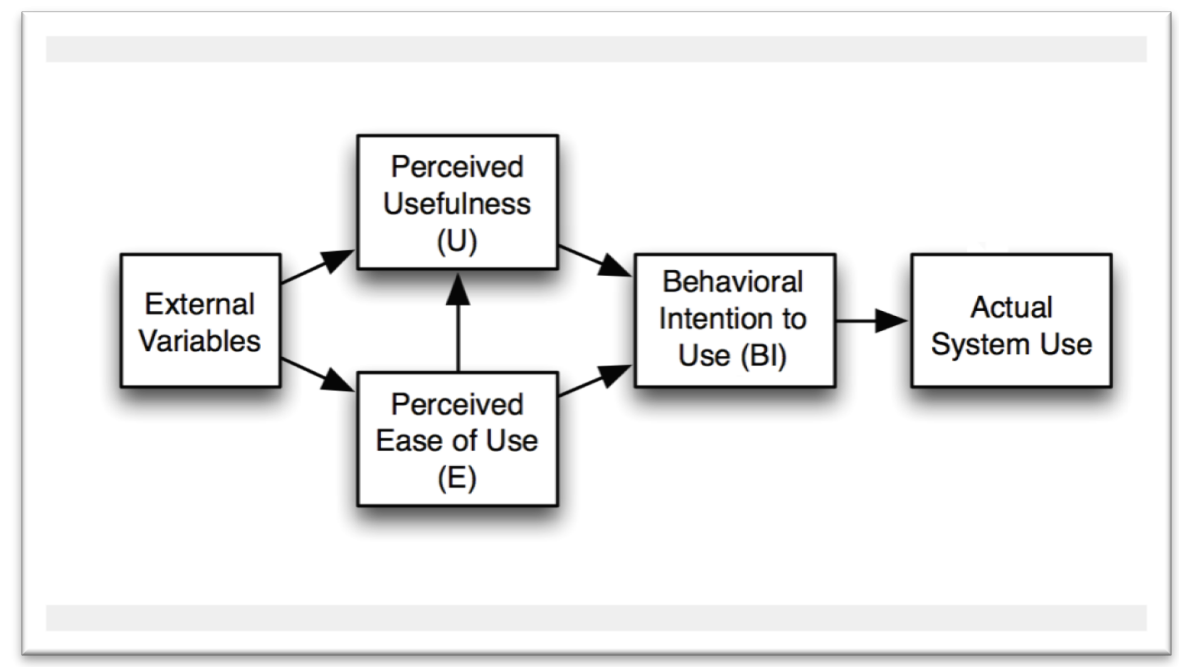

Figure 17 : Final version of Technology Acceptance Model (TAM) [127].

\subsection{Research Model}

After reviewing the literature in telemedicine adoption, I grouped the adoption barriers to five main barriers: financial, technical, logistical, ethical and cultural. Each one of these five adoption barriers have sub-barriers. The hierarchical decision model (HDM) seems to be a good technique to solve this multi-criteria prioritization problem. The technology acceptance model (TAM) explains how a new technology and the various aspects of it are received and used by the user. The initial model is an integration of HDM and TAM.

The research model consists of eight expert panels:

\section{1) Expert panel 1: Technology Acceptance Model (TAM) perspective} Expert panel 1 focuses on the two main components of TAM regarding its 
perceived usefulness (refers to how much the user believes that the technology will help to improve the performance/efficiency) and perceived ease of use (refers to what extent the user is comfortable in using the features of the technology). The experts in this level should have a comprehensive understanding of the objectives provided and have expertise in the adoption process and theories.

\section{2) Expert panel 2: Main multiple perspective adoption barriers} Expert panel 2 focuses on main barriers of levels of the hierarchy and consists of executive managers and people who are in higher managerial positions in the healthcare field and able to select the best alternatives for adoption of tele consultation for the care of the senior population. The experts in this level should have a comprehensive understanding of the objectives provided and should be able to contribute to the main objectives of the research.

\section{3) Expert panel 3 - Financial barriers}

Expert panel 3 analyzes financial barriers like startup cost, ongoing cost, resource allocation and net benefits. The experts for this panel have significant experience in the field of economics and finance as well as hospital administration. Those experts consist of hospital finance administrators and professors from academia. 


\section{4) Expert panel 4 - Technical barriers}

Expert panel 4 has a broad knowledge and experience of technical characteristics of the healthcare field and particularly telemedicine technology like computer skills, infrastructure and technical support. This panel consists of engineers, technologists, hospital IT managers and specialists.

\section{5) Expert panel 5 - Logistical barriers}

Expert panel 5 focuses on assessing the logistical barriers of organizational subcriteria like the regulation issues involved in implementation of telemedicine, licensure, credentialing and malpractice. Those experts have significant experience in project management, personnel management, technology management and human resource allocation. The experts come from healthcare organizations and academia.

\section{6) Expert panel 6 - Ethical barriers}

Expert of panel 6 focuses on the ethical issues that rise in the adoption of telemedicine like privacy and security, beneficence and justice. This panel consists of doctors, nurses and IT specialists with experience in the field of telemedicine technology. They should be able to assess patients' benefits factors and their privacy and security issue. This group of experts has experience in doctor-patient, nurse-patient and doctor-nurse communication patterns as well has a good understanding of issues of sharing information. 


\section{7) Expert panel 7- Cultural barriers}

Expert panel 7 focuses on issues of acceptance and satisfaction of using telemedicine services by physicians, nurse practitioners, physician assistants and patients. The main expertise is provided by doctors, nurses and IT specialists. This group of experts has experience in doctor-patient, nurse-patient and doctor-nurse communication patterns as well has a good understanding of issues of sharing information.

\section{8) Expert panel 8 - Alternatives level}

Expert panel 7 focuses on several alternative solutions to address adoption barriers. This panel consists of engineers, technologists, project/program managers and people familiar with impacts of telemedicine in healthcare who could evaluate it in respect to the subcriteria provided.

The expert panels focus and required expertise for the initial research are summarized in Table 8 below: 


\begin{tabular}{|c|c|c|}
\hline Panel no & Focus & Required expertise \\
\hline Panel 1 & $\begin{array}{l}\text { Technology Acceptance } \\
\text { Model (TAM) perspective }\end{array}$ & $\begin{array}{l}\text { Executive managers with expertise in } \\
\text { adoption process and theories. }\end{array}$ \\
\hline Panel 2 & $\begin{array}{l}\text { Main multiple perspective } \\
\text { adoption barriers }\end{array}$ & Top decision level and program managers \\
\hline Panel 3 & Financial barriers & $\begin{array}{c}\text { Hospital financial services managers and } \\
\text { account administrators. }\end{array}$ \\
\hline Panel 4 & Technical barriers & $\begin{array}{l}\text { Health information technology, technical } \\
\text { support Engineers and medical } \\
\text { technologists. }\end{array}$ \\
\hline Panel 5 & Logistical barriers & $\begin{array}{l}\text { Healthcare Logistics, policy and } \\
\text { Regulation managers. }\end{array}$ \\
\hline Panel 6 & Ethical barriers & $\begin{array}{c}\text { Ethics and committee consultation service } \\
\text { managers }\end{array}$ \\
\hline Panel 7 & Cultural barriers & $\begin{array}{l}\text { Health information technology, Doctors \& } \\
\text { nurses and patient relations/patient } \\
\text { resources departments mangers. }\end{array}$ \\
\hline Panel 8 & Alternatives level & $\begin{array}{c}\text { Engineers, technologists, Doctors \& } \\
\text { nurses and patient relations. }\end{array}$ \\
\hline
\end{tabular}

Table 8: Expert panel focus and required expertise

\subsubsection{Integrated Model}

The output of the HDM is a prioritized ranking indicating the overall preference for each of the decision alternatives. The HDM can be used to solve prioritization problems. To address the research objectives and research gaps, a theoretical research model is developed as shown in Figure 18. The initial research model is based on a literature review of barriers related to the adoption and use of teleconsultation. Also, the initial research model is based on a literature review of HDM and TAM models to develop an appropriate research model. 


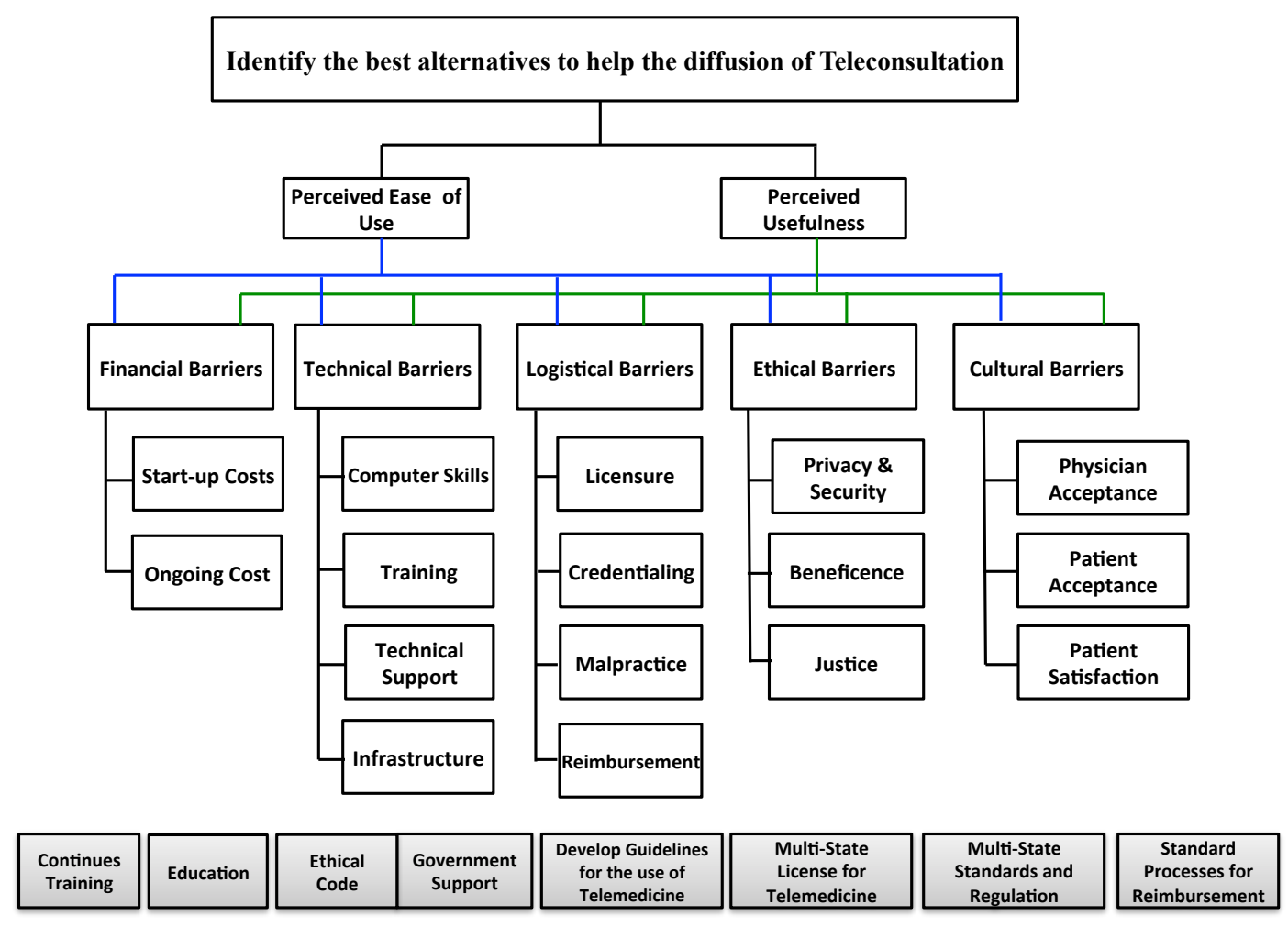

Figure 18 : Initial research model.

\subsubsection{Model validation}

After the initial model was developed, a number of web-based content validity instruments were developed. Model validation was conducted through 8 content validity instruments that were focused on different parts of the assessment model. A total of 44 experts from different backgrounds and expertise were identified through several selection methods as shown in Table 9 below. 


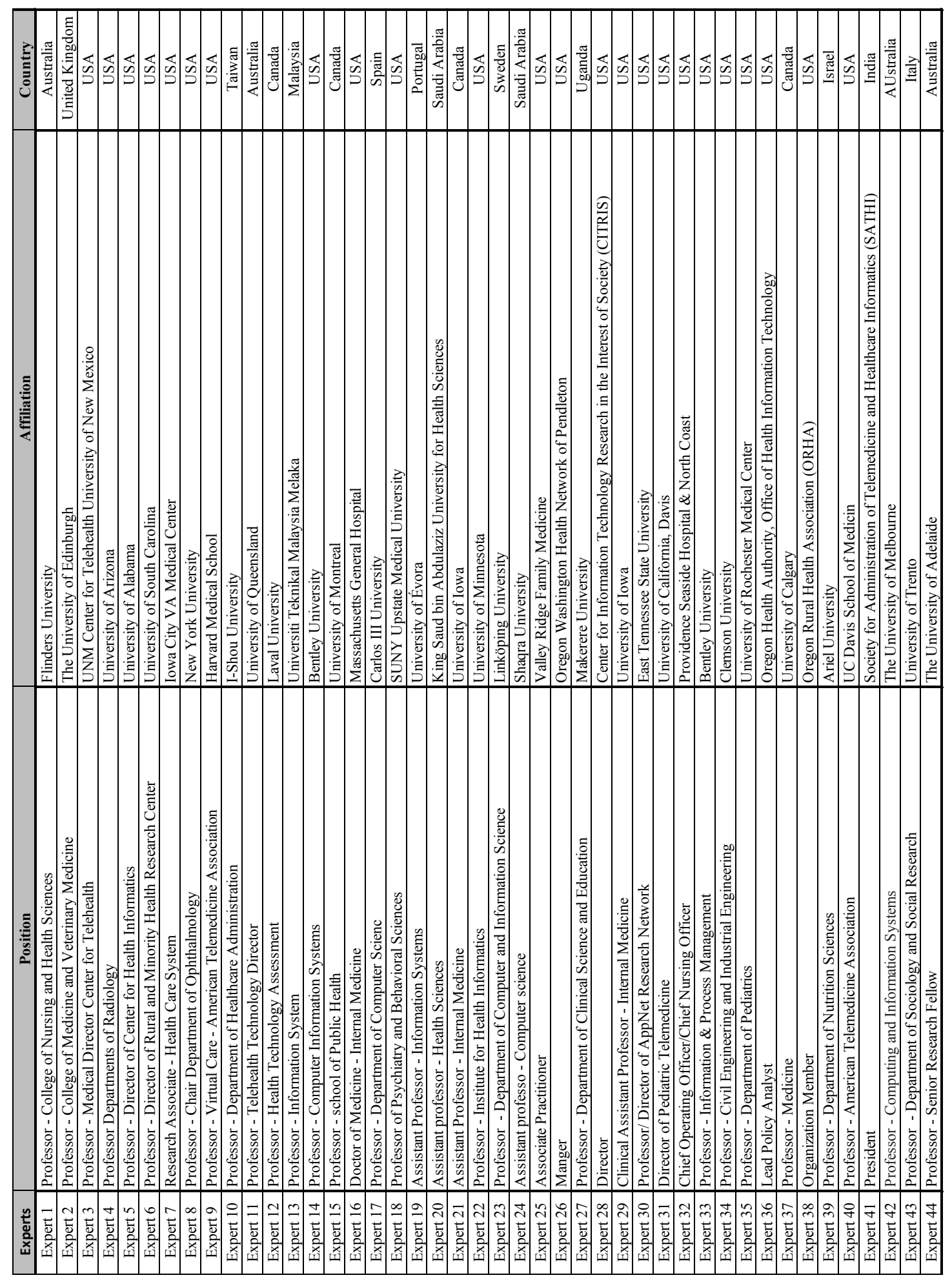

Table 9 : Total of experts and their background. 
A total of 34 experts were distributed over the 8 content validity instruments based on their backgrounds and expertise, and some of them participated in more than one panel because of their expertise. Please see Table 10 below for content validity instruments and sizes for judgment quantification.

\begin{tabular}{|c|c|c|}
\hline Instruments & Focus & $\begin{array}{c}\text { Number of } \\
\text { Participants }\end{array}$ \\
\hline Content validity instrument 1 & $\begin{array}{c}\text { Technology Acceptance Model } \\
\text { (TAM) perspective }\end{array}$ & 10 \\
\hline Content validity instrument 2 & $\begin{array}{c}\text { Main multiple perspective } \\
\text { adoption barriers }\end{array}$ & 10 \\
\hline Content validity instrument 3 & Financial barriers & 8 \\
\hline Content validity instrument 4 & Technical barriers & 7 \\
\hline Content validity instrument 5 & Logistical barriers & 11 \\
\hline Content validity instrument 6 & Ethical barriers & 12 \\
\hline Content validity instrument 7 & Cultural barriers & 8 \\
\hline Content validity instrument 8 & Alternatives level & 8 \\
\hline
\end{tabular}

Table 10: Focus and number of participants per content validity instrument

In order for a variable to be included in the assessment model, at least two thirds $(<67 \%)$ of the experts on a panel had to agree on its suitability [163]. Accordingly, a large majority of the respondents for most of content validity instruments agreed that the proposed variables were suitable for this research. The tables below show the content validity results for each content validity instrument.

Content validity instrument 1 focused on validating the suitability of the two components of TAM in measuring the mission. A total of 10 experts provided input and 10 out of 10 said "Yes" for both of the components of TAM, perceived ease of use and perceived usefulness. Please refer to Appendix $C$ for 
content validity instrument 1 . Please see Table 11 below for a summary of responses.

\begin{tabular}{|c|c|c|c|c|c|}
\hline \multirow{2}{*}{ Focus } & \multirow{2}{*}{ Variables } & \multicolumn{3}{c|}{ Responses } & $\begin{array}{c}\text { Agreement } \\
\text { Level }\end{array}$ \\
\cline { 3 - 6 } & & Yes & No & Total & $100 \%$ \\
\hline $\begin{array}{c}\text { Technology Acceptance } \\
\text { Model (TAM) }\end{array}$ & Perceived Ease of Use & 10 & 0 & 10 & $100 \%$ \\
\cline { 2 - 6 } & Perceived Usefulness & 10 & 0 & 10 & 10 \\
\hline
\end{tabular}

Table 11 : Summary of responses for content validity instrument 1

Content validity instrument 2 focused on validating the five main multiple perspective adoption barriers. A total of 10 experts provided input. Please refer to Appendix $\mathrm{C}$ for content validity instrument 2 . Four out of the 5 adoption barriers were within the acceptance range and only one adoption barrier was less than the acceptance range $<67 \%$. Five out of $10(50 \%)$ experts said 'NO' for the ethical barriers. The ethical barriers did not meet the two thirds of experts' agreement, so it was omitted from the model. Please see Table 12 below for a summary of the responses.

\begin{tabular}{|c|c|c|c|c|c|}
\hline \multirow{2}{*}{ Focus } & \multirow{2}{*}{ Variables } & \multicolumn{3}{|c|}{ Responses } & \multirow{2}{*}{$\begin{array}{c}\text { Agreement } \\
\text { Level }\end{array}$} \\
\hline & & Yes & No & Total & \\
\hline \multirow{5}{*}{$\begin{array}{c}\text { Main multiple } \\
\text { perspective adoption } \\
\text { barriers }\end{array}$} & Financial Barriers & 7 & 3 & 10 & $70 \%$ \\
\hline & Technical Barriers & 10 & $\mathbf{0}$ & 10 & $100 \%$ \\
\hline & Logistical Barriers & 9 & 1 & 10 & $90 \%$ \\
\hline & Ethical Barriers & 5 & 5 & 10 & $50 \%$ \\
\hline & Cultural Barriers & 7 & 3 & 10 & $70 \%$ \\
\hline
\end{tabular}

Table 12 : Summary of responses for content validity instrument 2

Content validity instrument 3 focused on validating sub-criteria under financial barriers. A total of 8 experts provided input. Both of the 2 subcriteria were within the acceptance range $<67 \%$. Please refer to Appendix $C$ for content validity instrument 3. Please see Table 13 below for a summary of responses. 


\begin{tabular}{|c|c|c|c|c|c|}
\hline \multirow{2}{*}{ Focus } & \multirow{2}{*}{ Variables } & \multicolumn{3}{|c|}{ Responses } & Agreement \\
& & Yes & No & Total & Level \\
\hline \multirow{2}{*}{ Financial Barriers } & Start-up Cost & $\mathbf{8}$ & $\mathbf{0}$ & $\mathbf{8}$ & $100 \%$ \\
\cline { 2 - 6 } & Ongoing Cost & 7 & 1 & $\mathbf{8}$ & $\mathbf{8 8 \%}$ \\
\hline
\end{tabular}

Table 13 : Summary of responses for content validity instrument 3

Content validity instrument 4 focused on validating subcriteria under technical barriers. A total of 7 experts provided input. All 4 subcriteria were within the acceptance range $<67 \%$. Please refer to Appendix $C$ for content validity instrument 4. Please see Table 14 below for a summary of responses.

\begin{tabular}{|c|c|c|c|c|c|}
\hline \multirow{2}{*}{ Focus } & \multirow{2}{*}{ Variables } & \multicolumn{3}{|c|}{ Responses } & $\begin{array}{c}\text { Agreement } \\
\text { Level }\end{array}$ \\
\cline { 2 - 6 } & & Yes & No & Total & $86 \%$ \\
\hline \multirow{3}{*}{ Technical Barriers } & Computer Skills & 6 & 1 & 7 & $86 \%$ \\
\cline { 2 - 6 } & Training & 6 & 1 & 7 & $86 \%$ \\
\cline { 2 - 6 } & Technical Support & 6 & 1 & 7 & $100 \%$ \\
\cline { 2 - 6 } & Infrastructure & 7 & 0 & 7 & 8 \\
\hline
\end{tabular}

Table 14 : Summary of responses for content validity instrument 4

Content validity instrument 5 focused on validating subcriteria under logistical barriers. A total of 11 experts provided input. All 4 subcriteria were within the acceptance range $<67 \%$. Please refer to Appendix $C$ for content validity instrument 5 . Please see Table 15 below for a summary of responses.

\begin{tabular}{|c|c|c|c|c|c|}
\hline \multirow{2}{*}{ Focus } & \multirow{2}{*}{ Variables } & \multicolumn{3}{|c|}{ Responses } & $\begin{array}{c}\text { Agreement } \\
\text { Level }\end{array}$ \\
\cline { 2 - 6 } & & Yes & No & Total & $73 \%$ \\
\hline \multirow{3}{*}{ Logistical Barriers } & Licensure & $\mathbf{8}$ & 3 & 11 & $73 \%$ \\
\cline { 2 - 6 } & Credentialing & $\mathbf{8}$ & 3 & 11 & $91 \%$ \\
\cline { 2 - 6 } & Malpractice & 10 & 1 & 11 & $91 \%$ \\
\cline { 2 - 6 } & Reimbursement & 10 & 1 & 11 & 73 \\
\hline
\end{tabular}

Table 15: Summary of responses for content validity instrument 5

Content validity instrument 6 focused on validating sub-criteria under ethical barriers. A total of 12 experts provided input. Only 1 subcriteria, privacy \& security, 
was within the acceptance range $<67 \%$. The experts suggested to add privacy \& security under the technical barriers. Two out of the 3 subcriteria were less than the acceptance range $<67 \%$. Six out of $12(50 \%)$ experts said 'NO' for beneficence. Five out of 12 (42\%) experts said 'NO' for justice. Beneficence and Justice did not meet the two thirds of experts' agreement, so they were omitted from the model. Please see Table 16 below for a summary of responses. Please refer to Appendix C for content validity instrument 6 .

\begin{tabular}{|c|c|c|c|c|c|}
\hline \multirow{2}{*}{ Focus } & \multirow{2}{*}{ Variables } & \multicolumn{3}{|c|}{ Responses } & Agreement \\
& & Yes & No & Total & Level \\
\cline { 2 - 6 } & Privacy \& Security & 10 & 2 & 12 & $83 \%$ \\
\cline { 2 - 6 } Ethical Barriers & Beneficence & 6 & 6 & 12 & $50 \%$ \\
\cline { 2 - 6 } & Justice & 7 & 5 & 12 & $58 \%$ \\
\hline
\end{tabular}

Table 16 : Summary of responses for content validity instrument 6

Content validity instrument 7 focused on validating sub-criteria under cultural barriers. A total of 8 experts provided input. All 3 subcriteria were within the acceptance range $<67 \%$. Please refer to Appendix $C$ for content validity instrument 7 . Please see Table 17 below for a summary of responses.

\begin{tabular}{|c|c|c|c|c|c|}
\hline \multirow{2}{*}{ Focus } & \multirow{2}{*}{ Variables } & \multicolumn{3}{|c|}{ Responses } & Agreement \\
\cline { 3 - 6 } & & Yes & No & Total & Level \\
\hline \multirow{3}{*}{ Cultural Barriers } & Physician Acceptance & 7 & 1 & $\mathbf{8}$ & $\mathbf{8 8 \%}$ \\
\cline { 2 - 6 } & Patient Acceptance & 7 & 1 & $\mathbf{8}$ & $\mathbf{8 8 \%}$ \\
\cline { 2 - 6 } & Patient Satisfaction & $\mathbf{7}$ & $\mathbf{1}$ & $\mathbf{8}$ & $\mathbf{8 8 \%}$ \\
\hline
\end{tabular}

Table 17 : Summary of responses for content validity instrument 7

Content validity instrument 8 focused on validating alternative solutions. A total of 8 experts provided input. Seven alternative solutions were within the acceptance range $<67 \%$ and only 1 alternative solution was less than the acceptance range $<67 \%$. Three out of $8(37 \%)$ experts said 'NO' for ethical code. Ethical code did not meet the two thirds of experts' agreement, so it was omitted 
from the model. Please refer to Appendix $C$ for content validity instrument 8 . Please see Table 18 below for a summary of responses.

\begin{tabular}{|c|c|c|c|c|c|}
\hline \multirow{2}{*}{ Focus } & \multirow{2}{*}{ Variables } & \multicolumn{3}{|c|}{ Responses } & \multirow{2}{*}{$\begin{array}{c}\text { Agreement } \\
\text { Level }\end{array}$} \\
\cline { 2 - 6 } & Continues Training & 7 & 1 & 8 & $88 \%$ \\
\hline & Education & 8 & 0 & 8 & $100 \%$ \\
\cline { 2 - 6 } & Ethical Code & 5 & 3 & 8 & $63 \%$ \\
\cline { 2 - 6 } & Government Support & 8 & 0 & 8 & $100 \%$ \\
\cline { 2 - 6 } & $\begin{array}{c}\text { Develop Guidelines for } \\
\text { the use of Telemedicine }\end{array}$ & 8 & 0 & 8 & $100 \%$ \\
\cline { 2 - 6 } & $\begin{array}{c}\text { Multi-State License for } \\
\text { Telemedicine }\end{array}$ & 7 & 1 & 8 & $88 \%$ \\
\cline { 2 - 6 } & $\begin{array}{c}\text { Multi-State Standards } \\
\text { and Regulation }\end{array}$ & 8 & 0 & 8 & $100 \%$ \\
\cline { 2 - 6 } & $\begin{array}{c}\text { Standard Processes for } \\
\text { Reimbursement }\end{array}$ & 7 & 1 & 8 & $88 \%$ \\
\hline
\end{tabular}

Table 18: Summary of responses for content validity instrument 8

\subsubsection{The finalized research model}

Based on the content validity results, the revised research model was modified, and the final research model was obtained as shown in Figure 19. The finalized research model consists of 4 levels: mission statement, main multiple perspective adoption barriers, subfactors, and alternative solutions. 


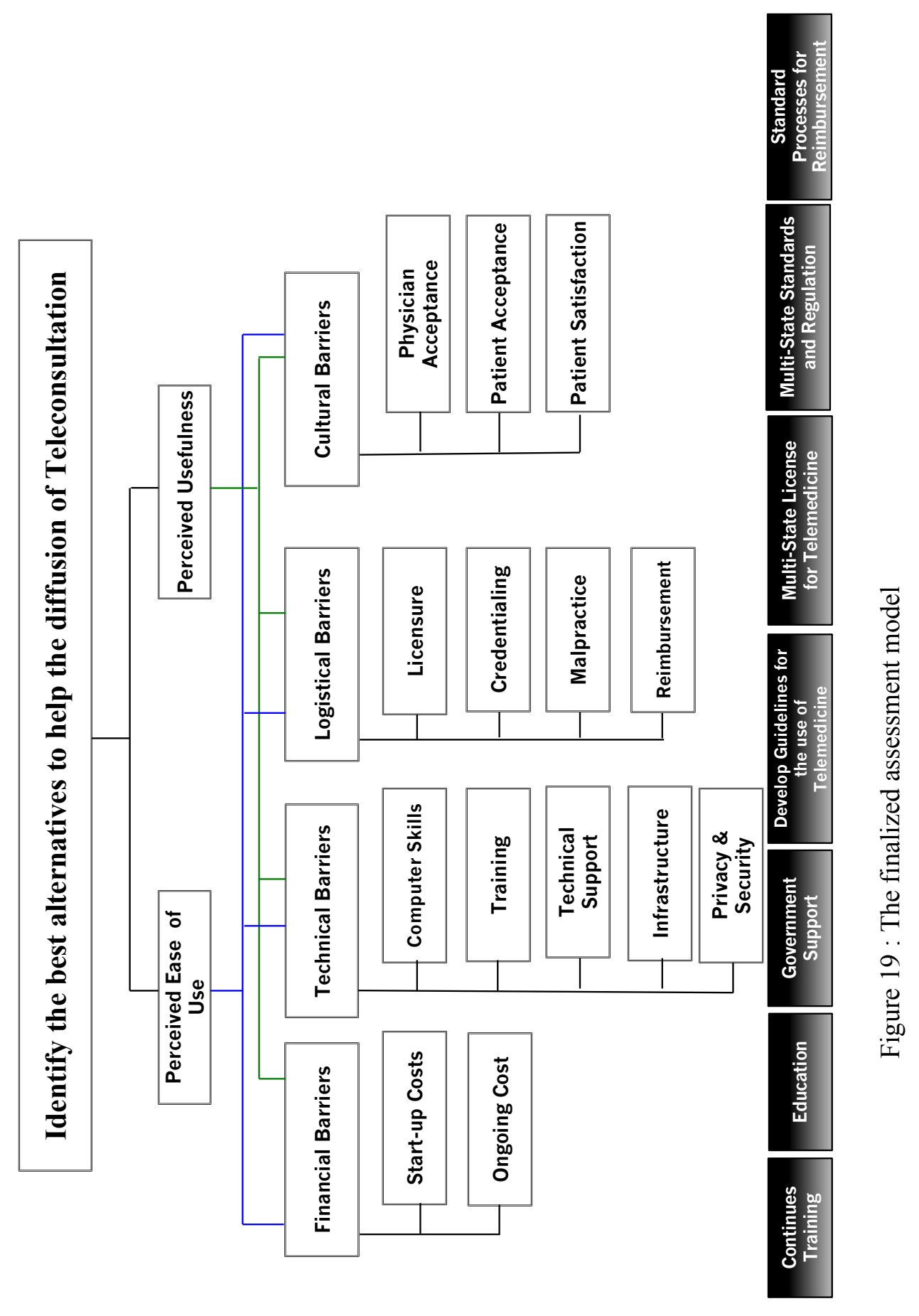




\subsection{Judgment quantifications}

The Judgment quantification was conducted using 7 panels, which were focused on different parts of the assessment model. Each panel required different types of expertise. The choice of judgment quantification method for panels was the pairwise comparison method.

A total of 23 experts with various backgrounds and positions participated in the judgment quantification process. Experts had experience in the areas of management, planning, engineering, and economics. Experts were distributed over the 7 panels based on their backgrounds and expertise and some of them participated in more than one panel because of their expertise.

1) Expert panel 1 consisted of 7 experts and focused on quantifying the relative importance of the 2 components of technology acceptance model (TAM), perceived ease of use and perceived usefulness. Please refer to Appendix $\mathrm{D}$ for the judgment quantification instrument for panel 1 .

2) Expert panel 2 consisted of 5 experts and focused on quantifying the relative importance of the 4 main multiple perspective adoption barriers; financial technical, logistical, and cultural barriers. Please refer to Appendix D for the judgment quantification instrument for panel 2.

3) Expert panel 3 consisted of 6 experts and focused on quantifying the relative importance of subcriteria under financial barriers; startup cost and 
ongoing cost. Please refer to Appendix $D$ for the judgment quantification instrument for panel 3.

4) Expert panel 4 consisted of 7 experts and focused on quantifying the relative importance of subcriteria under technical barriers; computer skills, training, technical support, infrastructure, and privacy and security. Please refer to Appendix $D$ for the judgment quantification instrument for panel 4 .

5) Expert panel 5 consisted of 5 experts and focused on quantifying the relative importance of subcriteria under logistical barriers; licensure, credentialing, malpractice, and reimbursement. Please refer to Appendix D for the judgment quantification instrument for panel 5.

6) Expert panel 6 consisted of 8 experts and focused on quantifying the relative importance of subcriteria under cultural barriers: physician acceptance, patient acceptance, and patient satisfaction. Please refer to Appendix $D$ the judgment quantification instrument for panel 6 .

7) Expert panel 7 consisted of 5 experts and focused on quantifying the relative importance of the alternative solutions with respect to each sub criterion: continues training, education, government support, develop guidelines for the use of telemedicine, multi-state license for telemedicine, multi-state standards and regulation, standard processes for reimbursement. Please refer to Appendix $D$ for the judgment quantification instrument for panel 7. 


\section{CHAPTER 5: DATA ANALYSIS AND RESULTS}

In this chapter, judgment quantification results, expert inconsistencies, and group disagreements are presented for each panel. Followed by the panel results in this synthesis of the priorities, and sensitivity analyses.

Quantified expert judgments were analyzed using a pairwise comparison method software. Based on the previous studies, the threshold value used for determining expert inconsistencies and group disagreements was 0.10 [164]. Results are presented in the order of the expert panels.

\subsection{Expert panel 1}

\subsubsection{Expert panel 1 results}

Expert panel 1 consisted of 7 experts, who evaluated the relative importance of the two components of technology acceptance model (TAM), perceived ease of use and perceived usefulness, with respect to the mission statement. Based on 7 experts, the arithmetic means of the relative importance of the perceived ease of use and perceived usefulness are shown in the Figure 20 below. 
TAM

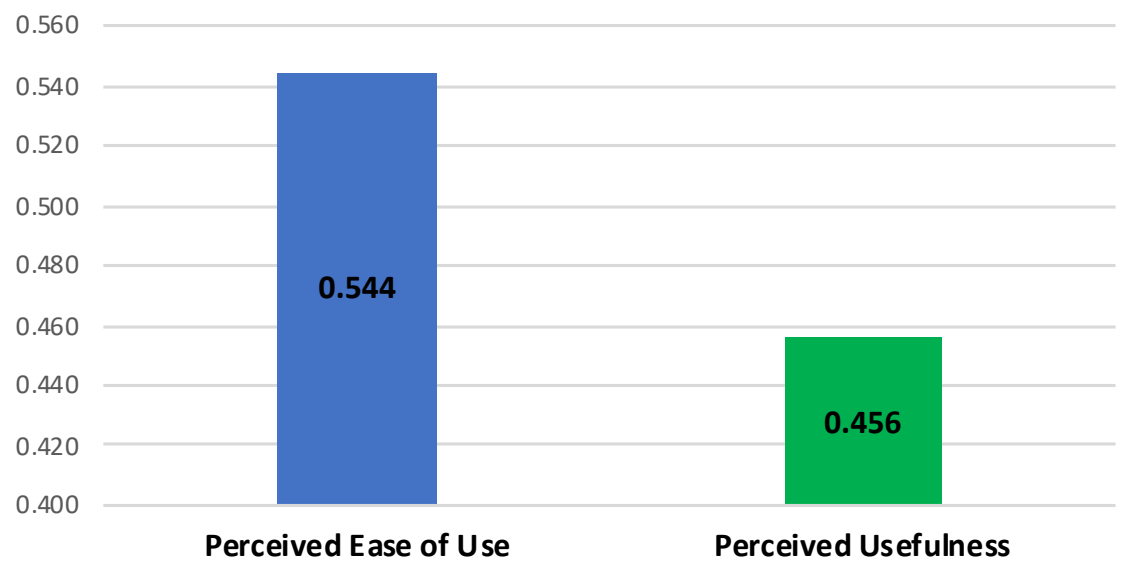

Figure 20 : Relative importance of perceived ease of use and perceived usefulness

According to the results, perceived ease of use $(54.4 \%)$ is the most important components technology acceptance model with respect to the mission. Perceived usefulness (45.6\%) has almost the same relative importance.

\subsubsection{Analysis of expert panel 1 results}

According to panel 1 results, all of the experts reflect an acceptable level of consistency in their judgments. There is a significant level of disagreement among the experts (0.108). Experts' individual relative priorities, inconsistency levels, aggregated group results, and group disagreement values are shown in Table 19 below. 


\begin{tabular}{|c|c|c|c|}
\hline Expert & Perceived Ease of Use & Perceived Usefulness & Inconsistency \\
\hline Expert 1 & 0.6 & 0.4 & 0 \\
\hline Expert 2 & 0.5 & 0.5 & 0 \\
\hline Expert 3 & 0.5 & 0.5 & 0 \\
\hline Expert 9 & 0.81 & 0.19 & 0 \\
\hline Expert 12 & 0.6 & 0.4 & 0 \\
\hline Expert 16 & 0.45 & 0.55 & 0 \\
\hline Expert 20 & 0.35 & 0.65 & 0 \\
\hline Mean & 0.54 & 0.46 & \\
\hline Minimum & 0.35 & 0.19 & \\
\hline Maximum & 0.81 & 0.65 & \\
\hline Std. Deviation & 0.13 & 0.13 & \\
\hline Disagreement & & & 0.108 \\
\hline
\end{tabular}

Table 19 : Analysis of expert panel 1 results

Subgroup analysis has identified 2 subgroups within expert panel 1. Please see Figure 21 below for details. Group disagreement indices for each subgroup; subgroup A (0.05) and subgroup B (0.093) are lower than the threshold value of 0.10. Experts 'individual relative priorities, inconsistency levels, aggregated group results, and group disagreement indices for each subgroup are shown in the tables below. 


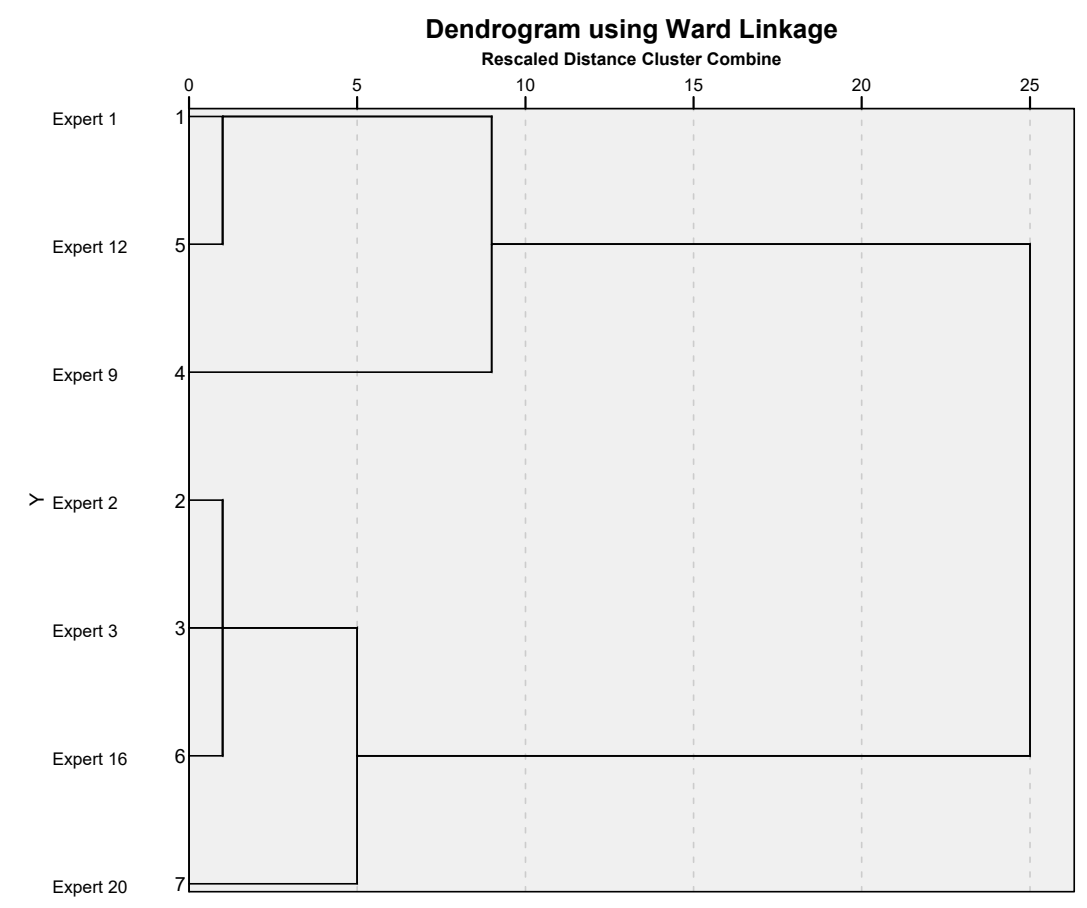

Figure 21 : Subgroups in expert panel 1 using dendrogram

Subgroup "A" is the largest group in expert panel 1 and consists of 4 experts. Experts in subgroup "A" placed perceived usefulness (55.0\%) first and followed by perceived ease of use (45.0\%) which is almost the opposite of the total panel weight as shown in table 20 below.

\begin{tabular}{|c|c|c|c|}
\hline Expert & Perceived Ease of Use & Perceived Usefulness & Inconsistency \\
\hline Expert 2 & 0.50 & 0.50 & 0 \\
\hline Expert 3 & 0.50 & 0.50 & 0 \\
\hline Expert 16 & 0.45 & 0.55 & 0 \\
\hline Expert 20 & 0.35 & 0.65 & 0 \\
\hline Mean & 0.45 & 0.55 & \\
\hline Minimum & 0.35 & 0.50 & \\
\hline Maximum & 0.50 & 0.65 & \\
\hline Std. Deviation & 0.06 & 0.06 & \\
\hline Disagreement & & & 0.050 \\
\hline
\end{tabular}

Table 20 : Analysis of Subgroup "A" results in expert panel 1 
Subgroup "B" is the smallest group in expert panel 1 and consists of 3 experts. Experts in subgroup "B" placed perceived ease of use $(67.0 \%)$ first, followed by perceived usefulness $(33.0 \%)$, which is higher than the total panel weight as shown in Table 21 below.

\begin{tabular}{|c|c|c|c|}
\hline Expert & Perceived Ease of Use & Perceived Usefulness & Inconsistency \\
\hline Expert 1 & 0.6 & 0.4 & 0 \\
\hline Expert 9 & 0.81 & 0.19 & 0 \\
\hline Expert 12 & 0.60 & 0.40 & 0 \\
\hline Mean & 0.67 & 0.33 & \\
\hline Minimum & 0.60 & 0.19 & \\
\hline Maximum & 0.81 & 0.40 & \\
\hline Std. Deviation & 0.10 & 0.10 & \\
\hline Disagreement & & & 0.093 \\
\hline
\end{tabular}

Table 21 : Analysis of Subgroup "B" results in expert panel 1

\subsection{Expert panel 2}

\subsubsection{Expert panel 2 results}

Expert panel 2 consisted of 5 experts who evaluated the relative importance of the 4 main multiple perspective adoption barriers: financial, technical, logistical, and cultural barriers.

\subsubsection{Perceived ease of use}

Based on 5 experts, the arithmetic means of the relative importance of the four main multiple perspective adoption barriers: financial, technical, logistical, and cultural barriers are shown in the Figure 22 below. 


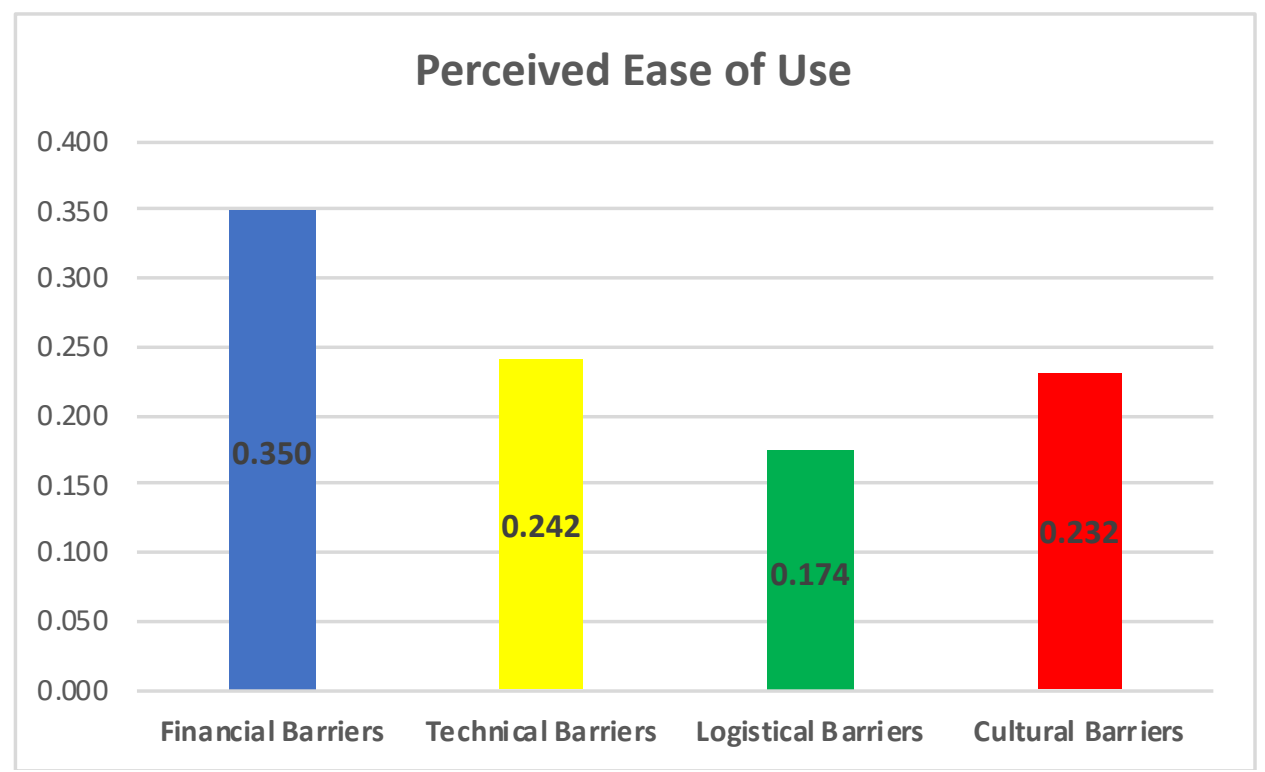

Figure 22 : Relative importance of criteria with respect to the mission (A)

According to the results, financial barriers (35.0\%) are the most important adoption barriers with respect to the mission. Technical barriers $(24.2 \%)$ and cultural barriers $(23.2 \%)$ have almost the relative importance and rank second and third correspondingly. Logistical barriers (17.4\%) are the least important adoption barriers and the category ranks fourth.

\subsubsection{Perceived usefulness}

Based on 5 experts, the arithmetic means of the relative importance of the 4 main multiple perspective adoption barriers: financial, technical, logistical, and cultural barriers are shown in the Figure 23 below. 


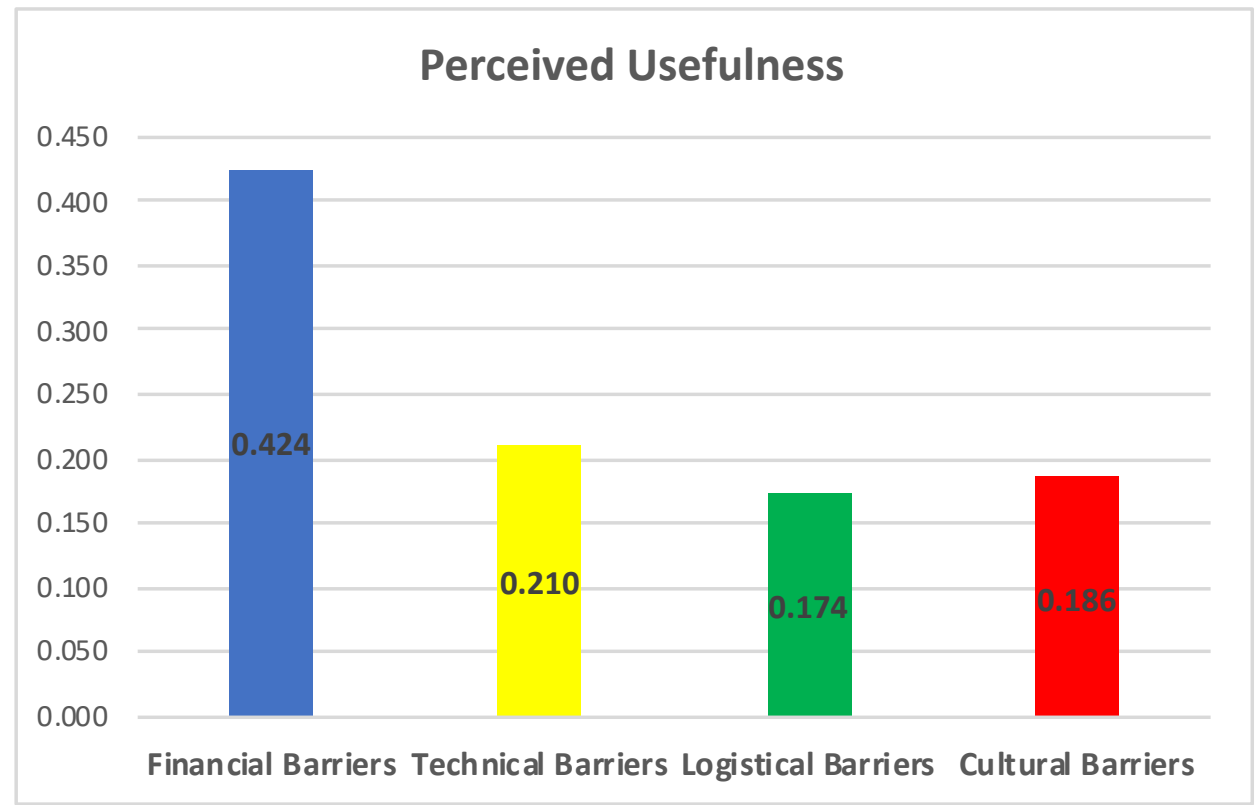

Figure 23 : Relative importance of criteria with respect to the mission (B)

According to the results, financial barriers (42.4\%) are the most important adoption barriers with respect to the mission. Technical barriers (21.0\%), cultural barriers (18.6\%), and logistical barriers (17.4\%) have almost the same relative importance and rank second, third and fourth correspondingly.

\subsubsection{Analysis of expert panel 2 results}

\subsubsection{Perceived ease of use}

According to panel 2 results, all of the experts reflect an acceptable level of consistency in their judgments. Also, there is no significant level of disagreement among the experts (0.088). Experts' individual relative priorities, inconsistency levels, aggregated group results, and group disagreement values are shown in Table 22 below. 


\begin{tabular}{|c|c|c|c|c|c|}
\hline & \multicolumn{3}{|c|}{ Perceived Ease of Use } & \multirow[b]{2}{*}{ Cultural Barriers } & \multirow[b]{2}{*}{ Inconsistency } \\
\hline Expert & Financial Barriers & Technical Barriers & Logistical Barriers & & \\
\hline Expert 5 & 0.13 & 0.36 & 0.32 & 0.18 & 0.05 \\
\hline Expert 7 & 0.44 & 0.32 & 0.12 & 0.12 & 0.01 \\
\hline Expert 8 & 0.43 & 0.12 & 0.10 & 0.35 & 0.01 \\
\hline Expert 11 & 0.39 & 0.25 & 0.19 & 0.17 & 0.00 \\
\hline Expert 21 & 0.36 & 0.16 & 0.14 & 0.34 & 0.08 \\
\hline Mean & 0.35 & 0.24 & 0.17 & 0.23 & \\
\hline Minimum & 0.13 & 0.12 & 0.10 & 0.12 & \\
\hline Maximum & 0.44 & 0.36 & 0.32 & 0.35 & \\
\hline Std. Deviation & 0.11 & 0.09 & 0.08 & 0.09 & \\
\hline Disagreement & & & & & 0.088 \\
\hline
\end{tabular}

Table 22 : Analysis of expert panel 2 (A) results

\subsubsection{Perceived usefulness}

According to panel 2 results, all of the experts reflect an acceptable level of consistency in their judgments. Also, there is no significant level of disagreement among the experts (0.072). Experts' individual relative priorities, inconsistency levels, aggregated group results, and group disagreement values are shown in Table 23 below.

\begin{tabular}{|c|c|c|c|c|c|}
\cline { 2 - 6 } \multicolumn{1}{c|}{} & \multicolumn{3}{|c|}{ Perceived Ease of Use } & & Inconsistency \\
\hline Expert & Financial Barriers & \multicolumn{1}{c|}{ Technical Barriers } & \multicolumn{1}{c|}{ Logistical Barriers } & Cultural Barriers & Inco. \\
\hline Eta S. Berner & 0.57 & 0.18 & 0.18 & 0.06 & 0.00 \\
\hline ane Moeckli & 0.42 & 0.33 & 0.12 & 0.12 & 0.01 \\
\hline Joel Schuman & 0.45 & 0.12 & 0.17 & 0.25 & 0.07 \\
\hline Liam Caffery & 0.39 & 0.25 & 0.19 & 0.17 & 0.01 \\
\hline Spyridon Fortis & 0.29 & 0.17 & 0.21 & 0.33 & 0.00 \\
\hline Mean & 0.42 & 0.21 & 0.17 & 0.19 & \\
\hline Minimum & 0.29 & 0.12 & 0.12 & 0.06 & \\
\hline Maximum & 0.57 & 0.33 & 0.21 & 0.33 & \\
\hline Std. Deviation & 0.09 & 0.07 & 0.03 & 0.10 & 0.072 \\
\hline Disagreement & & & & & \\
\hline
\end{tabular}

Table 23 : Analysis of expert panel 2 (B) results 


\subsection{Expert panel 3}

\subsubsection{Expert panel 3 results}

Expert panel 3 consisted of 6 experts who evaluated the relative importance of subcriteria under financial barriers: startup cost and ongoing cost. Based on 6 experts, the arithmetic means of the relative importance of the startup cost and ongoing cost are shown in the Figure 24 below.

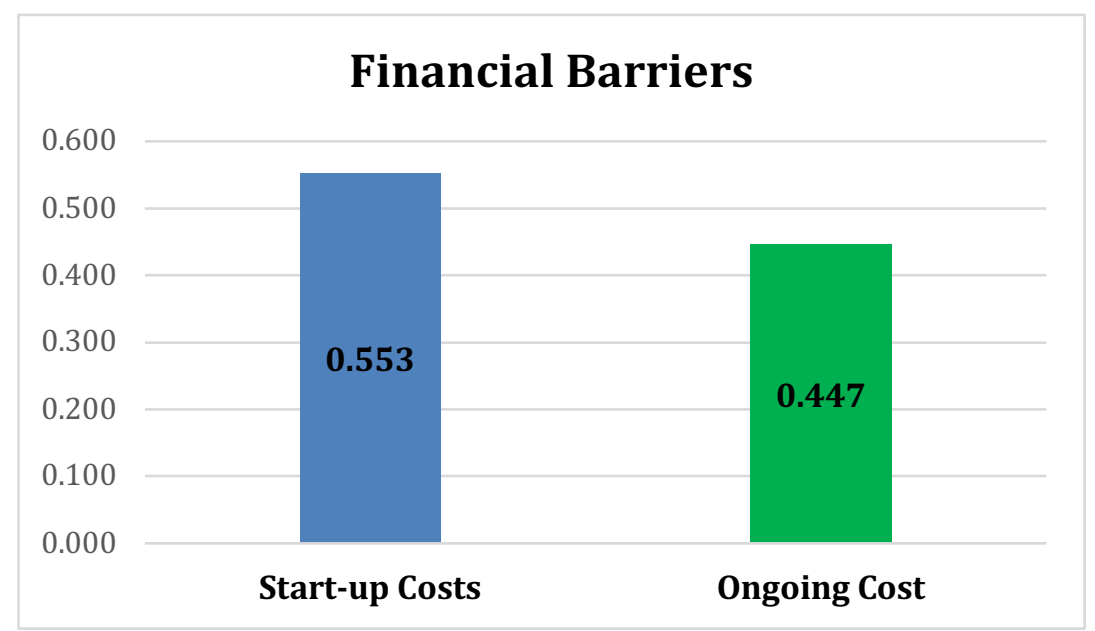

Figure 24 : Relative importance of sub-criteria under financial barriers

According to the results, startup cost $(55.3 \%)$ is the most important subcriteria with respect to the financial barriers. Ongoing cost $(44.7 \%)$ is the second most important subcriteria with respect to the financial barriers, and it has almost the same relative importance.

\subsubsection{Analysis of expert panel 3 results}

According to panel 3 result, all of the experts reflect an acceptable level of consistency in their judgments. There is a significant level of disagreement among the experts (0.133). Experts' individual relative priorities, inconsistency levels, 
aggregated group results, and group disagreement values are shown in Table 24 below.

\begin{tabular}{|c|c|c|c|}
\hline Expert & Start-up Costs & Ongoing Cost & Inconsistency \\
\hline Expert 2 & 0.35 & 0.65 & 0.00 \\
\hline Expert 3 & 0.75 & 0.25 & 0.00 \\
\hline Expert 5 & 0.41 & 0.59 & 0.00 \\
\hline Expert 12 & 0.50 & 0.50 & 0.00 \\
\hline Expert 16 & 0.56 & 0.44 & 0.00 \\
\hline Expert 20 & 0.75 & 0.25 & 0.00 \\
\hline Mean & 0.55 & 0.45 & \\
\hline Minimum & 0.35 & 0.25 & \\
\hline Maximum & 0.75 & 0.65 & \\
\hline Std. Deviation & 0.15 & 0.15 & 0.133 \\
\hline Disagreement & & &
\end{tabular}

Table 24 : Analysis of expert panel 3 results

Subgroup analysis has identified 2 subgroups within expert panel 3 . Please see the Figure 25 below for details. Group disagreement indices for each subgroup; subgroup "A" (0.075) and subgroup "B" (0.000) are lower than the threshold value of 0.10 . Experts' individual relative priorities, inconsistency levels, aggregated group results, and group disagreement indices for each subgroup are shown in the tables below. 


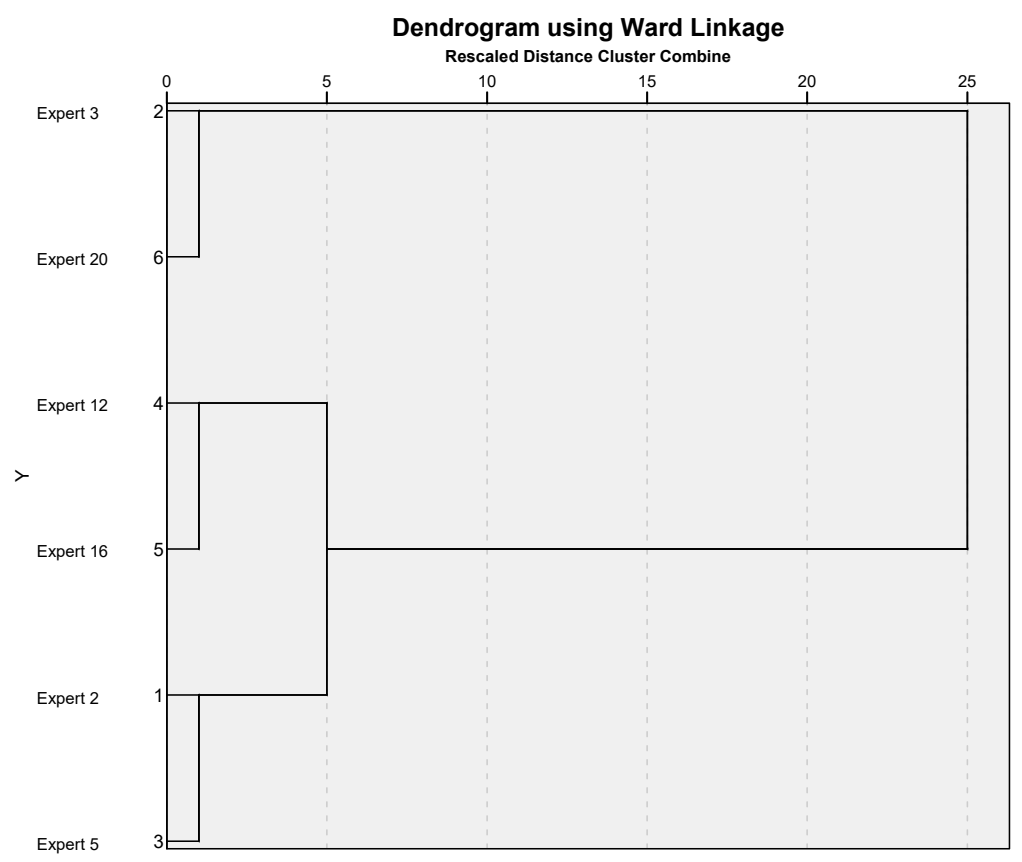

Figure 25 : Subgroups in expert panel 3 using dendrogram

Subgroup "A" is the largest group in expert panel 3 and consists of 4 experts. Experts in subgroup "A" placed ongoing cost $(54.5 \%)$ first, followed by startup cost $(45.5 \%)$, which is the opposite of the total panel weight as shown in Table 25 below.

\begin{tabular}{|c|c|c|c|}
\hline Expert & Start-up Costs & Ongoing Cost & Inconsistency \\
\hline Expert 2 & 0.35 & 0.65 & 0.00 \\
\hline Expert 5 & 0.41 & 0.59 & 0.00 \\
\hline Expert 12 & 0.50 & 0.50 & 0.00 \\
\hline Expert 16 & 0.56 & 0.44 & 0.00 \\
\hline Mean & 0.455 & 0.545 & \\
\hline Minimum & 0.35 & 0.44 & \\
\hline Maximum & 0.56 & 0.65 & \\
\hline Std. Deviation & 0.08 & 0.08 & \\
\hline Disagreement & & & 0.075 \\
\hline
\end{tabular}

Table 25 : Analysis of Subgroup "A" results in expert panel 3 
Subgroup "B" is the smallest group in expert panel 3 and consists of 2 experts. Experts in subgroup "B" placed startup cost (75.0\%) first, followed by ongoing cost $(25.0 \%)$, which is matching the same ranks of the total panel as shown in Table 26 below.

\begin{tabular}{|c|c|c|c|}
\hline Expert & Start-up Costs & Ongoing Cost & Inconsistency \\
\hline Expert 3 & 0.75 & 0.25 & 0.00 \\
\hline Expert 20 & 0.75 & 0.25 & 0.00 \\
\hline Mean & 0.75 & 0.25 & \\
\hline Minimum & 0.75 & 0.25 & \\
\hline Maximum & 0.75 & 0.25 & \\
\hline Std. Deviation & 0.00 & 0.00 & 0.000 \\
\hline Disagreement & & & \\
\cline { 1 - 3 }
\end{tabular}

Table 26 : Analysis of Subgroup "B" results in expert panel 3

\subsection{Expert panel 4}

\subsubsection{Expert panel 4 results}

Expert panel 4 consisted of 7 experts, who evaluated the relative importance of subcriteria under technical barriers: computer skills, training, technical support, infrastructure and privacy and security. Based on 7 experts, the arithmetic means of the relative importance of computer skills, training, technical support, infrastructure and privacy and security are shown in the Figure 26 below. 


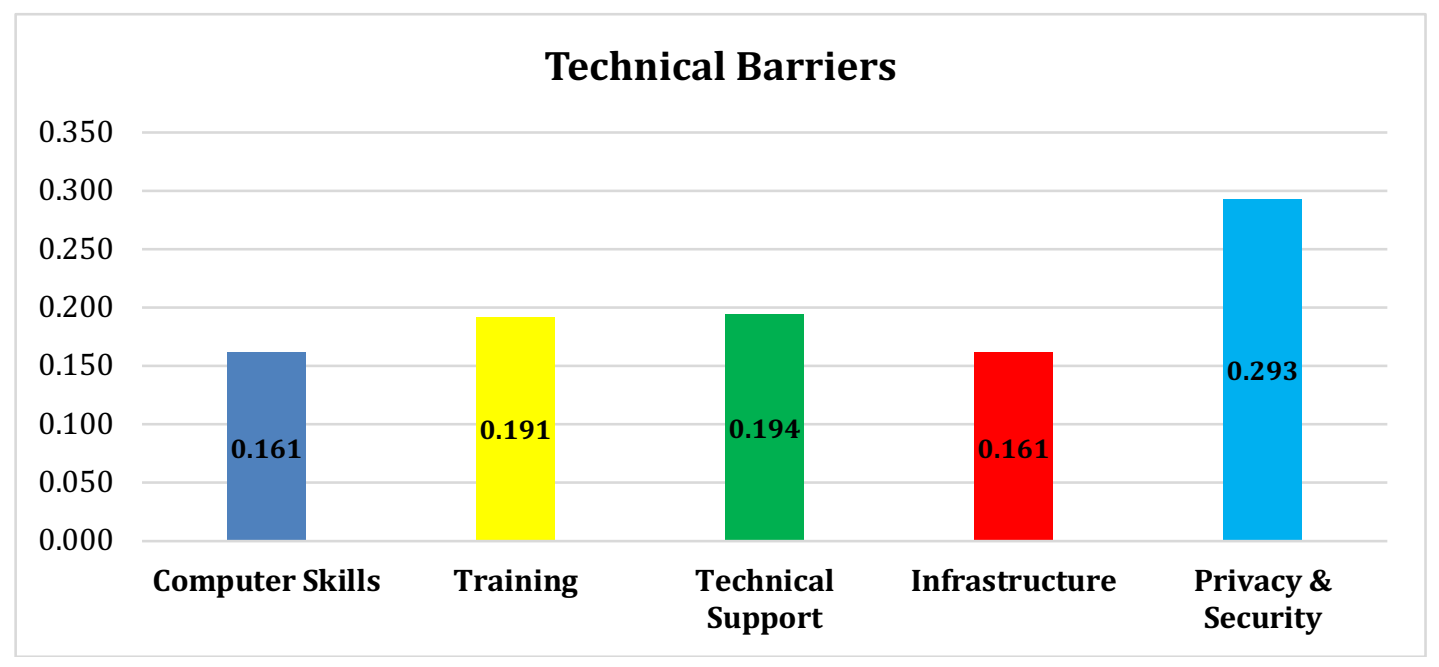

Figure 26 : Relative importance of sub-criteria under technical barriers

According to the results, privacy and security $(29.3 \%)$ are the most important subcriteria with respect to the technical barriers. Technical support (19.4\%) and training (19.1\%) have almost the same relative importance and rank second and third correspondingly. Computer skills (16.1\%) and infrastructure (16.1\%) have the same relative importance and ranked the least important subcriteria with respect to the technical barriers.

\subsubsection{Analysis of expert panel 4 results}

According to panel 4 results, all of the experts reflect an acceptable level of consistency in their judgments. Also, there is no significant level of disagreement among the experts (0.096). Experts' individual relative priorities, inconsistency levels, aggregated group results, and group disagreement values are shown in Table 27 below. 


\begin{tabular}{|c|c|c|c|c|c|c|}
\hline Expert & Computer Skills & Training & Technical Support & Infrastructure & Privacy \& Security & Inconsistency \\
\hline Expert 1 & 0.17 & 0.05 & 0.16 & 0.28 & 0.34 & 0.04 \\
\hline Expert 6 & 0.26 & 0.21 & 0.23 & 0.09 & 0.21 & 0.01 \\
\hline Expert 9 & 0.08 & 0.08 & 0.10 & 0.10 & 0.65 & 0.01 \\
\hline Expert 10 & 0.07 & 0.20 & 0.28 & 0.07 & 0.38 & 0.09 \\
\hline Expert 12 & 0.09 & 0.28 & 0.19 & 0.21 & 0.22 & 0.07 \\
\hline Expert 15 & 0.14 & 0.29 & 0.19 & 0.18 & 0.19 & 0.04 \\
\hline Expert 23 & 0.32 & 0.23 & 0.21 & 0.20 & 0.06 & 0.02 \\
\hline Mean & 0.161 & 0.191 & 0.194 & 0.161 & 0.293 & \\
\hline Minimum & 0.07 & 0.05 & 0.10 & 0.07 & 0.06 & \\
\hline Maximum & 0.32 & 0.29 & 0.28 & 0.28 & 0.65 & \\
\hline Std. Deviation & 0.09 & 0.09 & 0.05 & 0.07 & 0.17 & \\
\hline Disagreement & & & & & & 0.096 \\
\hline
\end{tabular}

Table 27 : Analysis of expert panel 4 results

\subsection{Expert panel 5}

\subsubsection{Expert panel 5 results}

Expert panel 5 consisted of 5 experts who evaluated the relative importance of subcriteria under logistical barriers: licensure, credentialing, malpractice, and reimbursement. Based on 5 experts, the arithmetic means of the relative importance of licensure, credentialing, malpractice, and reimbursement are shown in the Figure 27 below.

\section{Logistical Barriers}

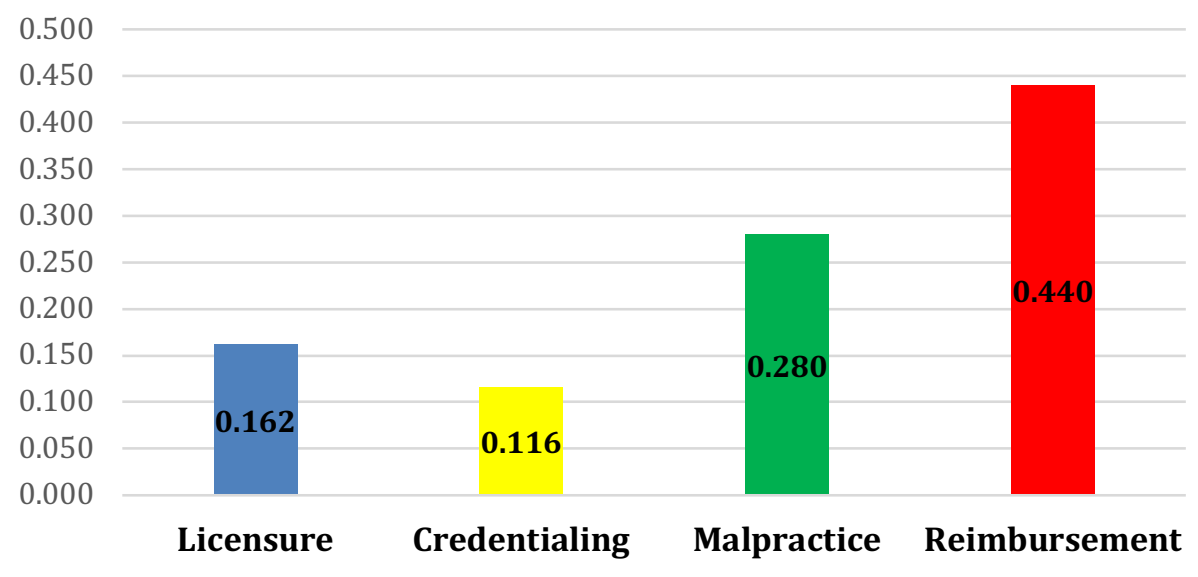

Figure 27 : Relative importance of subcriteria under logistical barriers 
According to the results, reimbursement $(44.0 \%)$ is the most important subcriteria with respect to the logistical barriers. Malpractice $(28.0 \%)$ is the second most important subcriteria with respect to the logistical barriers. Licensure $(16.2 \%)$ and credentialing (11.6\%) have almost the same relative importance and ranked the least important subcriteria with respect to the logistical barriers and placed third and fourth correspondingly.

\subsubsection{Analysis of expert panel 5 results}

According to panel 5 results, all of the experts reflect an acceptable level of consistency in their judgments. There is a significant level of disagreement among the experts (0.127). Experts' individual relative priorities, inconsistency levels, aggregated group results, and group disagreement values are shown in Table 28 below.

\begin{tabular}{|c|c|c|c|c|c|}
\hline Expert & Licensure & Credentialing & Malpractice & Reimbursement & Inconsistency \\
\hline Expert 7 & 0.25 & 0.25 & 0.25 & 0.25 & 0.00 \\
\hline Expert 8 & 0.17 & 0.05 & 0.17 & 0.60 & 0.01 \\
\hline Expert 15 & 0.18 & 0.08 & 0.26 & 0.48 & 0.07 \\
\hline Expert 19 & 0.13 & 0.16 & 0.57 & 0.14 & 0.00 \\
\hline Expert 21 & 0.08 & 0.04 & 0.15 & 0.73 & 0.06 \\
\hline Mean & 0.162 & 0.116 & 0.280 & 0.440 & \\
\hline Minimum & 0.08 & 0.04 & 0.15 & 0.14 & \\
\hline Maximum & 0.25 & 0.25 & 0.57 & 0.73 & \\
\hline Std. Deviation & 0.06 & 0.08 & 0.15 & 0.22 & \\
\hline Disagreement & & & & & 0.127 \\
\hline
\end{tabular}

Table 28 : Analysis of expert panel 5 results

Subgroup analysis has identified 2 subgroups within expert panel 5. Please see the Figure 28 below for details. Group disagreement indices for each subgroup: subgroup "A" (0.055) and subgroup "B" (0.093) are lower than the 
threshold value of 0.10 . Experts' individual relative priorities, inconsistency levels, aggregated group results, and group disagreement indices for each subgroup are shown in the tables below.

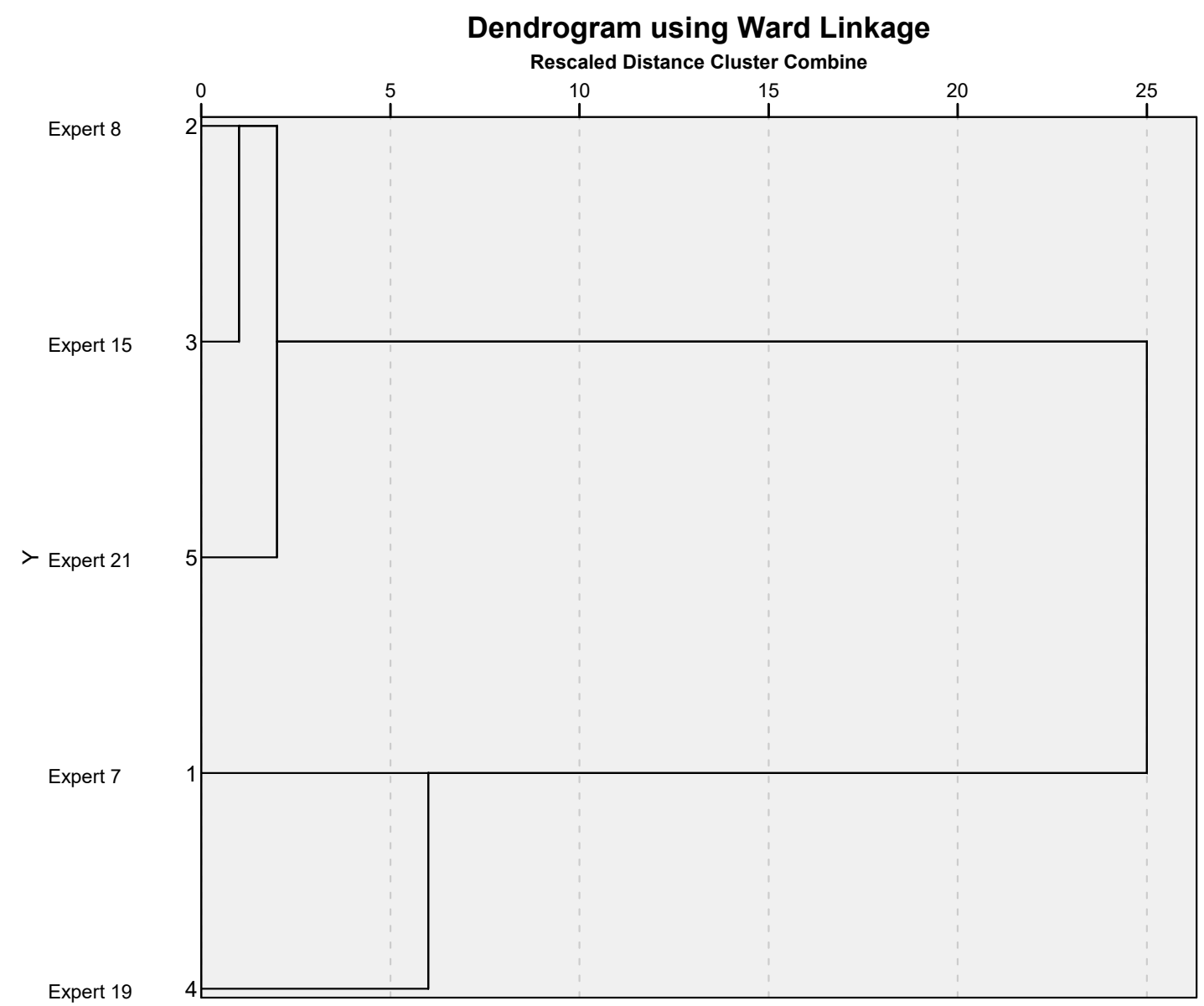

Figure 28 : Subgroups in expert panel 5 using dendrogram

Subgroup "A" is the largest group in expert panel 5 and consists of 3 experts. Experts in subgroup "A" placed reimbursement (60.3\%) first and followed by malpractice (19.3\%) and licensure (14.4\%) correspondingly, which is the same rank of the total panel weight. Credentialing $(5.7 \%)$ ranked the least important subcriteria with respect to the logistical barriers as shown in Table 29 below. 


\begin{tabular}{|c|c|c|c|c|c|}
\hline Expert & Licensure & Credentialing & Malpractice & Reimbursement & Inconsistency \\
\hline Expert 8 & 0.17 & 0.05 & 0.17 & 0.60 & 0.01 \\
\hline Expert 15 & 0.18 & 0.08 & 0.26 & 0.48 & 0.07 \\
\hline Expert 21 & 0.08 & 0.04 & 0.15 & 0.73 & 0.06 \\
\hline Mean & $\mathbf{0 . 1 4 3}$ & 0.057 & 0.193 & 0.603 & \\
\hline Minimum & $\mathbf{0 . 0 8}$ & 0.04 & 0.15 & 0.48 & \\
\hline Maximum & $\mathbf{0 . 1 8}$ & $\mathbf{0 . 0 8}$ & $\mathbf{0 . 2 6}$ & $\mathbf{0 . 7 3}$ & \\
\hline Std. Deviation & $\mathbf{0 . 0 4}$ & $\mathbf{0 . 0 2}$ & $\mathbf{0 . 0 5}$ & $\mathbf{0 . 1 0}$ & \\
\hline Disagreement & & & & & 0.055 \\
\hline
\end{tabular}

Table 29 : Analysis of Subgroup "A" results in expert panel 3

Subgroup "B" is the smallest group in expert panel 5 and consists of 2 experts. Experts in subgroup "B" placed malpractice (40.1\%) first, followed by credentialing (20.5\%) and reimbursement (19.5\%) correspondingly. Licensure $(19.0 \%)$ ranked the least important subcriteria with respect to the logistical barriers as shown in Table 30 below.

\begin{tabular}{|c|c|c|c|c|c|}
\hline Expert & Licensure & Credentialing & Malpractice & Reimbursement & Inconsistency \\
\hline Expert 7 & 0.25 & 0.25 & 0.25 & 0.25 & 0.00 \\
\hline Expert 19 & 0.13 & 0.16 & 0.57 & 0.14 & 0.00 \\
\hline Mean & 0.190 & 0.205 & 0.410 & 0.195 & \\
\hline Minimum & 0.13 & 0.16 & 0.25 & 0.14 & \\
\hline Maximum & 0.25 & 0.25 & 0.57 & 0.25 & \\
\cline { 1 - 4 } Std. Deviation & 0.06 & 0.04 & 0.16 & 0.06 & \\
\hline Disagreement & & & & & 0.093 \\
\hline
\end{tabular}

Table 30 : Analysis of Subgroup "B" results in expert panel 3

\subsection{Expert panel 6}

\subsubsection{Expert panel 6 results}

Expert panel 6 consisted of 7 experts who evaluated the relative importance of subcriteria under cultural barriers: physician acceptance, patient acceptance, and patient satisfaction. Based on 7 experts, the arithmetic means of the relative 
importance of physician acceptance, patient acceptance, and patient satisfaction are shown in the Figure 29 below.

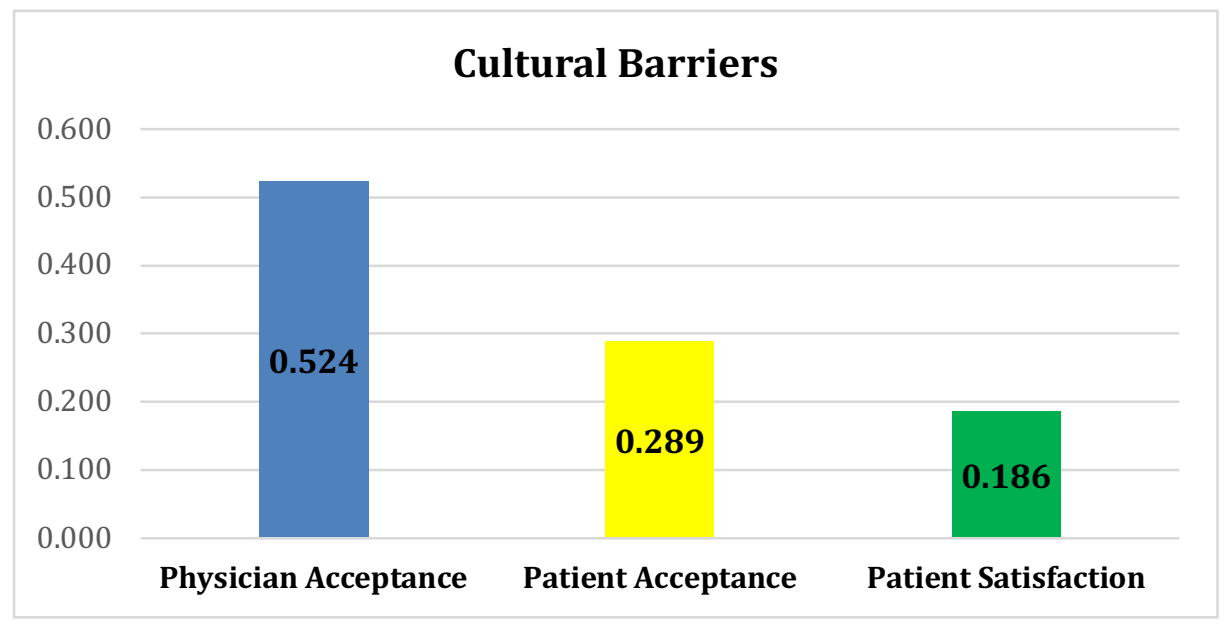

Figure 29 : Relative importance of sub-criteria under cultural barriers

According to the results, physician acceptance $(52.4 \%)$ is the most important sub-criteria with respect to the cultural barriers. Patient acceptance $(28.9 \%)$ is the second most important subcriteria with respect to the cultural barriers. Patient satisfaction (18.6\%) ranked the least important subcriteria with respect to the cultural barriers.

\subsubsection{Analysis of expert panel 6 results}

According to panel 6 results, all of the experts reflect an acceptable level of consistency in their judgments. There is a significant level of disagreement among the experts (0.139). Experts' individual relative priorities, inconsistency levels, aggregated group results, and group disagreement values are shown in Table 31 below. 


\begin{tabular}{|c|c|c|c|c|}
\hline Expert & Physician Acceptance & Patient Acceptance & Patient Satisfaction & Inconsistency \\
\hline Expert 4 & 0.45 & 0.34 & 0.21 & 0.00 \\
\hline Expert 12 & 0.33 & 0.33 & 0.33 & 0.00 \\
\hline Expert 13 & 0.30 & 0.53 & 0.17 & 0.09 \\
\hline Expert 14 & 0.67 & 0.18 & 0.16 & 0.00 \\
\hline Expert 17 & 0.58 & 0.14 & 0.28 & 0.04 \\
\hline Expert 19 & 0.44 & 0.44 & 0.11 & 0.00 \\
\hline Expert 22 & 0.90 & 0.06 & 0.04 & 0.01 \\
\hline Mean & 0.524 & 0.289 & 0.186 & \\
\hline Minimum & 0.30 & 0.06 & 0.04 & \\
\hline Maximum & 0.90 & 0.53 & 0.33 & \\
\hline Std. Deviation & 0.19 & 0.16 & 0.09 & 0.139 \\
\hline Disagreement & & & & \\
\hline
\end{tabular}

Table 31 : Analysis of expert panel 6 results

Subgroup analysis has identified 2 subgroups within expert panel 6 . Please see the Figure 30 below for details. Group disagreement indices for each subgroup: subgroup "A" (0.075) and subgroup "B" (0.091) are lower than the threshold value of 0.10 . Experts' individual relative priorities, inconsistency levels, aggregated group results, and group disagreement indices for each subgroup are shown in the tables below.

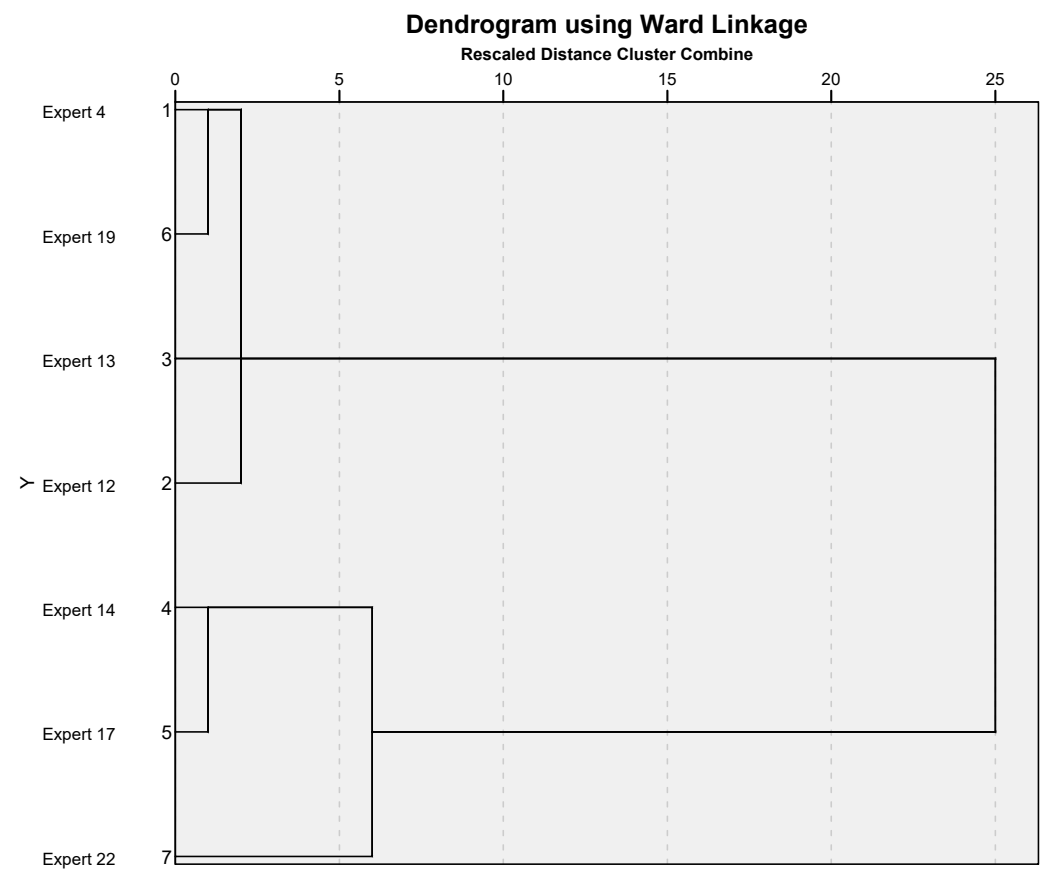

Figure 30 : Subgroups in expert panel 6 using dendrogram 
Subgroup "A" is the largest group in expert panel 6 and consists of 4 experts. Experts in subgroup "A" placed patient acceptance (41.0\%) first, followed by physician acceptance (38.0\%) and patient satisfaction (20.5\%) and rank second and third correspondingly as shown in Table 32 below.

\begin{tabular}{|c|c|c|c|c|}
\hline Expert & Physician Acceptance & Patient Acceptance & Patient Satisfaction & Inconsistency \\
\hline Expert 4 & 0.45 & 0.34 & 0.21 & 0.00 \\
\hline Expert 12 & 0.33 & 0.33 & 0.33 & 0.00 \\
\hline Expert 13 & 0.30 & 0.53 & 0.17 & 0.09 \\
\hline Expert 19 & 0.44 & 0.44 & 0.11 & 0.00 \\
\hline Mean & 0.380 & 0.410 & 0.205 & \\
\hline Minimum & 0.30 & 0.33 & 0.11 & \\
\hline Maximum & 0.45 & 0.53 & 0.33 & \\
\hline Std. Deviation & 0.07 & 0.08 & 0.08 & \\
\hline Disagreement & & & & 0.075 \\
\hline
\end{tabular}

Table 32 : Analysis of Subgroup "A" results in expert panel 6

Subgroup "B" is the smallest group in expert panel 6 and consists of 3 experts. Experts in subgroup "B" placed patient physician acceptance $(71.7 \%)$ first and followed by patient satisfaction (16.7\%) and patient acceptance $(12.0 \%)$ and rank second and third correspondingly as shown Table 33 in below.

\begin{tabular}{|c|c|c|c|c|}
\hline Expert & Physician Acceptance & Patient Acceptance & Patient Satisfaction & Inconsistency \\
\hline Expert 14 & $\mathbf{0 . 6 7}$ & $\mathbf{0 . 1 6}$ & $\mathbf{0 . 1 8}$ & 0.00 \\
\hline Expert 17 & $\mathbf{0 . 5 8}$ & $\mathbf{0 . 1 4}$ & $\mathbf{0 . 2 8}$ & 0.04 \\
\hline Expert 22 & $\mathbf{0 . 9 0}$ & $\mathbf{0 . 0 6}$ & $\mathbf{0 . 0 4}$ & 0.01 \\
\hline Mean & 0.717 & 0.120 & $\mathbf{0 . 1 6 7}$ & \\
\hline Minimum & $\mathbf{0 . 5 8}$ & $\mathbf{0 . 0 6}$ & $\mathbf{0 . 0 4}$ & \\
\hline Maximum & $\mathbf{0 . 9 0}$ & $\mathbf{0 . 1 6}$ & $\mathbf{0 . 2 8}$ & \\
\hline Std. Deviation & $\mathbf{0 . 1 3}$ & $\mathbf{0 . 0 4}$ & $\mathbf{0 . 1 0}$ & \\
\hline Disagreement & & & & 0.091 \\
\hline
\end{tabular}

Table 33 : Analysis of Subgroup "B" results in expert panel 6 


\subsection{Expert panel 7}

\subsubsection{Expert panel 7 results}

Expert panel 7 consisted of 5 experts, who evaluated the relative importance of the alternative solutions with respect to the mission. Based on 5 experts, the arithmetic means of the relative importance of continues training, education, government support, develop guidelines for the use of telemedicine, multi-state license for telemedicine, multi-state standards and regulation, and standard processes for reimbursement are shown in the Figure 31 below.

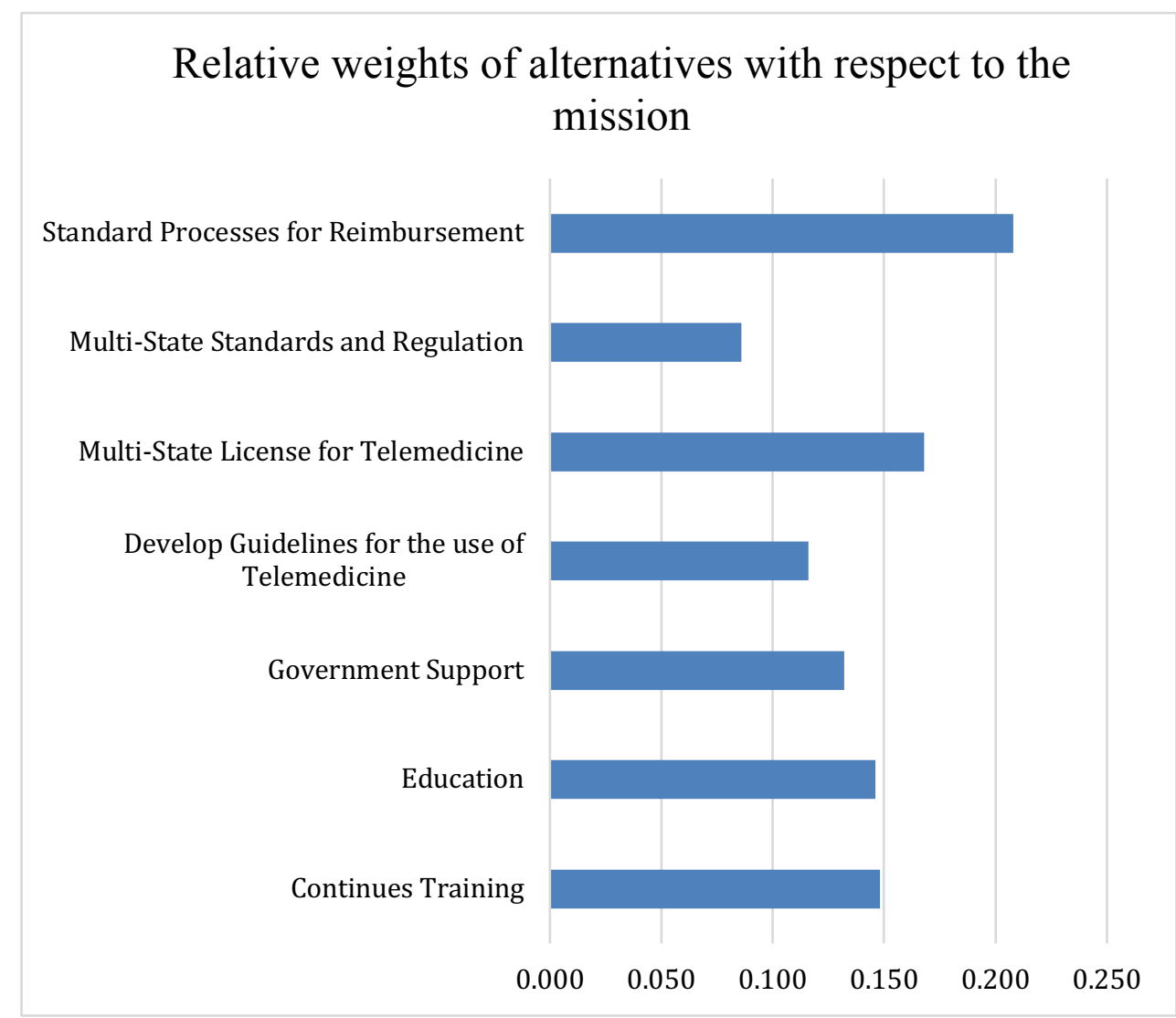

Figure 31 : Relative weights of alternatives with respect to the mission 
According to the results, standard processes for reimbursement $(20.8 \%)$ is the most important alternative solutions with respect to the mission. Multi-state license for telemedicine $(16.8 \%)$ is the second most important alternative solution with respect to the mission. Continues training (14.8\%) and education (14.6\%) are almost the same relative importance and ranked third and fourth correspondingly. Government support (13.2\%) ranked the fifth in importance and was followed by developing guidelines for the use of telemedicine which ranked the sixth in the importance. Multi-state standards and regulation (8.6\%) ranked the least important alternative solutions with respect to the mission.

\subsubsection{Analysis of expert panel 7 results}

According to panel 7 results, all of the experts reflect an acceptable level of consistency in their judgments. Also, there is no significant level of disagreement among the experts (0.068). Experts' individual relative priorities, inconsistency levels, aggregated group results, and group disagreement values are shown in Table 34 below.

\begin{tabular}{|c|c|c|c|c|c|c|c|c|}
\hline Expert & $\begin{array}{l}\text { Continues } \\
\text { Training }\end{array}$ & Education & $\begin{array}{c}\text { Government } \\
\text { Support }\end{array}$ & $\begin{array}{c}\text { Develop Guidelines } \\
\text { for the use of } \\
\text { Telemedicine }\end{array}$ & $\begin{array}{l}\text { Multi-State License } \\
\text { for Telemedicine }\end{array}$ & $\begin{array}{c}\text { Multi-State } \\
\text { Standards and } \\
\text { Regulation } \\
\end{array}$ & $\begin{array}{c}\text { Standard } \\
\text { Processes for } \\
\text { Reimbursement }\end{array}$ & Inconsistency \\
\hline Expert 4 & 0.1 & 0.16 & 0.15 & 0.05 & 0.15 & 0.04 & 0.35 & 0.06 \\
\hline Expert 14 & 0.1 & 0.06 & 0.16 & 0.05 & 0.27 & 0.08 & 0.29 & 0.06 \\
\hline Expert 18 & 0.11 & 0.09 & 0.06 & 0.13 & 0.25 & 0.15 & 0.21 & 0.05 \\
\hline Expert 23 & 0.26 & 0.27 & 0.16 & 0.12 & 0.08 & 0.07 & 0.05 & 0.09 \\
\hline Maximum & 0.26 & 0.27 & 0.16 & 0.23 & 0.27 & 0.15 & 0.35 & \\
\hline Std. Deviation & 0.06 & 0.07 & 0.04 & 0.07 & 0.08 & 0.04 & 0.11 & \\
\hline Disagreement & & & & & & & & 0.068 \\
\hline
\end{tabular}

Table 34 : Analysis of expert panel 7 results 


\subsection{Synthesis of Priorities}

According to the results of expert panels, synthesis of priorities is calculated for different levels of the decision hierarchy. The relative priorities of adoption barriers subcriteria with respect to the mission are calculated according to panel 1 results.

\subsubsection{Relative importance of subcriteria with respect to the mission}

The relative importance of all subcriteria, with respect to the mission, is analyzed in this section. This analysis gives more in-depth insight into the details of each criterion and can be useful for decision makers and analysts for future planning. The relative importance of all subcriteria with respect to the mission is shown in the Figure 32 below. 


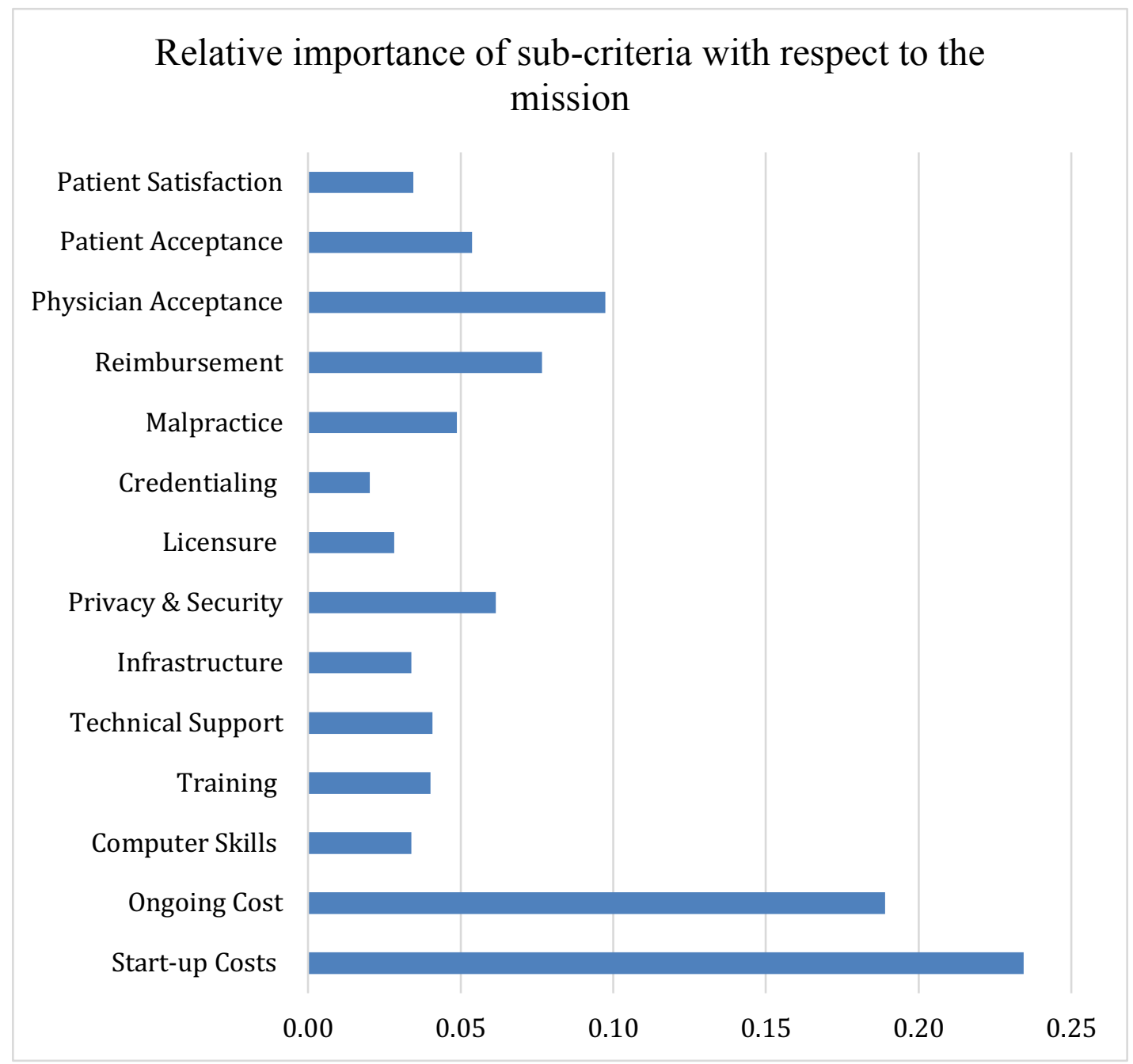

Figure 32 : Relative importance of sub-criteria with respect to the mission

The Startup Costs (0.23), ongoing cost (0.19), and physician acceptance $(0.10)$ are the top 3 highest weighted sub-criteria. It is important to remark that the top 2 subcriteria belong to the financial perspective. Physician acceptance $(0.19)$ is the next subcriteria highest weighted and it is a part of the cultural perspective.

Patient satisfaction (0.03), computer skills (0.03), infrastructure (0.03), licensure (0.03), and credentialing (0.02) are the lowest weighted subcriteria. It is important to remark that the least 2 subcriteria belong to the logistical perspective. 
The relative importance of all sub-criteria with respect to the mission are listed in Table 35 below.

\begin{tabular}{|l|c|c|}
\hline \multicolumn{1}{|c|}{ Sub-criteria } & Global weight & Rank \\
\hline Start-up Costs & 0.23 & 1 \\
\hline Ongoing Cost & 0.19 & 2 \\
\hline Physician Acceptance & 0.10 & 3 \\
\hline Reimbursement & 0.08 & 4 \\
\hline Privacy \& Security & 0.06 & 5 \\
\hline Patient Acceptance & 0.05 & 6 \\
\hline Malpractice & 0.05 & 6 \\
\hline Technical Support & 0.04 & 7 \\
\hline Training & 0.04 & 7 \\
\hline Patient Satisfaction & 0.03 & 8 \\
\hline Computer Skills & 0.03 & 8 \\
\hline Infrastructure & 0.03 & 8 \\
\hline Licensure & 0.03 & 8 \\
\hline Credentialing & 0.02 & 9 \\
\hline
\end{tabular}

Table 35 : The relative importance of all sub-criteria with respect to the mission

\subsubsection{Overall importance of alternative solutions with respect to the mission}

Global priorities of alternative solutions with respect to the mission are presented in this section. This analysis determines the ranking of the alternative solutions and identifies the solution that is most effective in increasing the adoption of teleconsultation according to the assessment perspectives. The overall importance of all alternative solutions with respect to the mission are shown in Figure 33 below. 


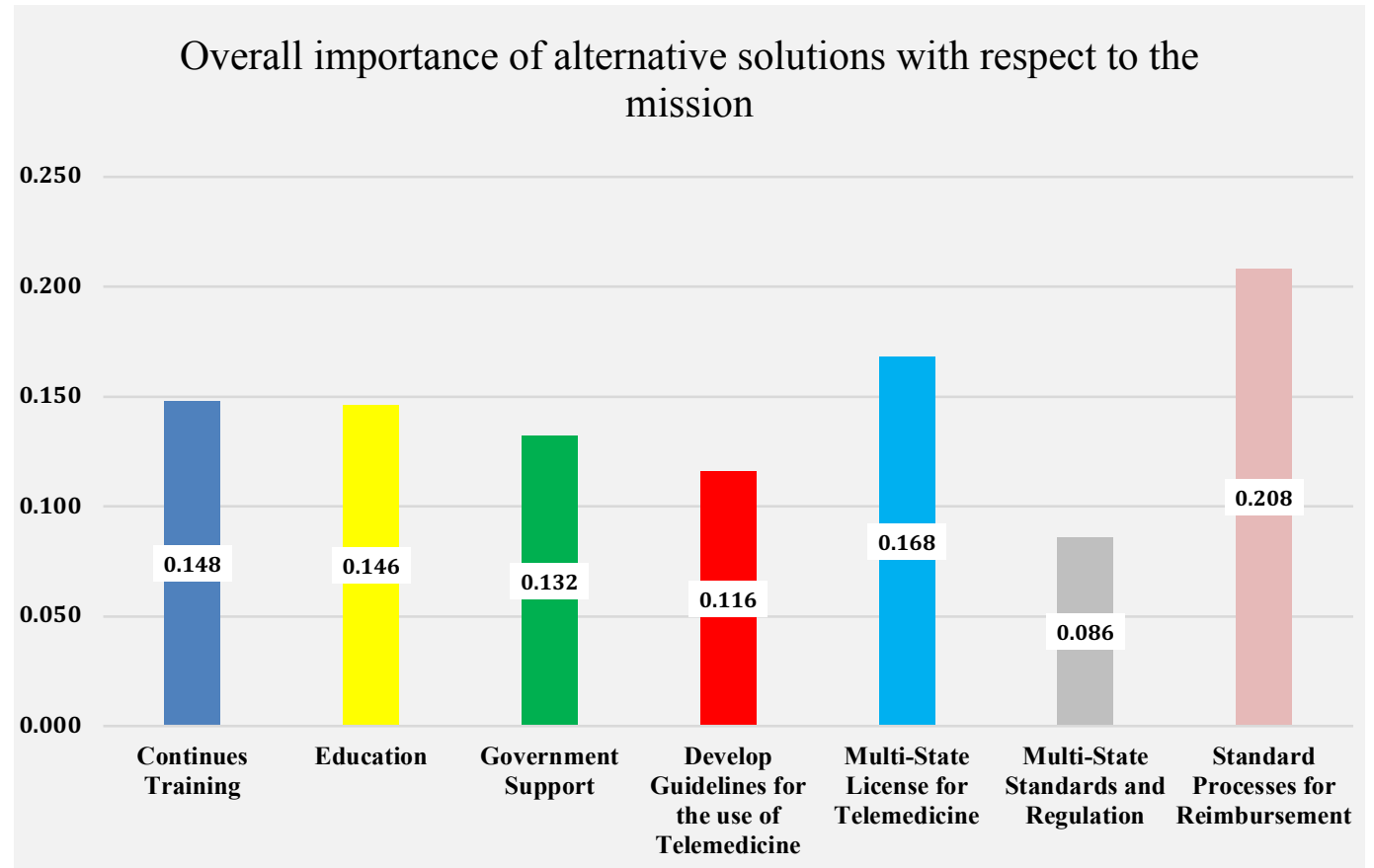

Figure 33 : Overall importance of alternative solutions with respect to the mission

According to the results, standard processes for reimbursement $(20.8 \%)$ is the most important alternative solutions with respect to the mission. Multi-state license for telemedicine $(16.8 \%)$ is the second most important alternative solution with respect to the mission. Continues training (14.8\%) and education (14.6\%) are almost the same relative importance and ranked third and fourth correspondingly. Government support (13.2\%) ranked the fifth in importance and was followed by developing guidelines for the use of telemedicine which ranked the sixth in importance. Multi-state standards and regulation (8.6\%) ranked the least important alternative solutions with respect to the mission. The overall importance of alternative solutions with respect to the mission are listed in Table 36 below. 


\begin{tabular}{|l|c|c|}
\hline \multicolumn{1}{|c|}{ Alternatives } & Global weight & Rank \\
\hline Standard Processes for Reimbursement & $20.80 \%$ & 1 \\
\hline Multi-State License for Telemedicine & $16.80 \%$ & 2 \\
\hline Continues Training & $14.80 \%$ & 3 \\
\hline Education & $14.60 \%$ & 4 \\
\hline Government Support & $13.20 \%$ & 5 \\
\hline Develop Guidelines for the use of Telemedicine & $11.60 \%$ & 6 \\
\hline Multi-State Standards and Regulation & $8.60 \%$ & 7 \\
\hline
\end{tabular}

Table 36 : The overall importance of alternative with respect to the mission

\subsection{Rank analysis of alternatives with respect to expert disagreements}

As discussed in previous sections, significant degrees of expert disagreements have been identified in Panel 3, Panel 5, and Panel 6; insights have been provided for each subgroup. This section attempts to explore whether or not expert disagreements have a significant impact on the overall rankings of alternatives. The overall rankings of alternatives are recalculated based on each subgroup response and compared with the original rankings, which are based on all experts' responses.

\subsubsection{Analysis of results with respect to expert disagreements in panel 3}

Experts in panel 3 disagree on the relative importance of subcriteria under the financial perspective. Hierarchical clustering analysis distinguished 2 subgroups in expert panel 3, which lowered the disagreement values. Table 37 below shows the relative importance of subcriteria per subgroups in expert panel 3. 


\begin{tabular}{|c|c|c|}
\hline Experts & Start-up cost & Ongoing cost \\
\hline Original panel 3 & 0.553 & 0.447 \\
\hline Subgroup "A" & 0.455 & 0.545 \\
\hline Subgroup "B" & 0.750 & 0.250 \\
\hline
\end{tabular}

Table 37 : Relative importance of subcriteria per subgroups in panel 3

The rankings of alternatives were recalculated according to the results of 2 subgroups and compared to original rankings of panel 3. Synthesis of priorities and rankings of alternatives with subgroups compared to overall panel 3 results are presented in Table 38 below.

\begin{tabular}{|l|c|c|c|}
\hline \multicolumn{1}{|c|}{ Alternatives } & Original panel 3 & Subgroup "A" & Subgroup "B" \\
\hline Standard Processes for Reimbursement & 1 & 1 & 1 \\
\hline Multi-State License for Telemedicine & 2 & 2 & 2 \\
\hline Continues Training & 3 & 3 & 3 \\
\hline Education & 4 & 4 & 4 \\
\hline Government Support & 5 & 5 & 5 \\
\hline Develop Guidelines for the use of Telemedicine & 6 & 6 & 6 \\
\hline Multi-State Standards and Regulation & 7 & 7 & 7 \\
\hline
\end{tabular}

Table 38 : Ranking of alternative solutions per subgroups in panel 3

The ranking of the alternative solutions remains unchanged with every subgroup compared to the complete panel 3 results. Standard processes for reimbursement still have the highest in importance ranking, followed by multi-state license for telemedicine, continues training, and education.

\subsubsection{Analysis of results with respect to expert disagreements in panel 5}

Experts in panel 5 disagree on the relative importance of subcriteria under the logistical perspective. Hierarchical clustering analysis distinguished two subgroups in expert panel 3 , which lowered the disagreement values. Table 39 
below shows the relative importance of subcriteria per subgroups in expert panel

5.

\begin{tabular}{|c|c|c|c|c|}
\hline Experts & Licensure & Credentialing & Malpractice & Reimbursement \\
\hline Original panel 5 & 0.162 & 0.116 & 0.280 & 0.440 \\
\hline Subgroup "A" & 0.143 & 0.057 & 0.193 & 0.603 \\
\hline Subgroup "B" & 0.190 & 0.205 & 0.410 & 0.195 \\
\hline
\end{tabular}

Table 39 : Relative importance of sub-criteria per subgroups in panel 5

The rankings of alternatives were recalculated according to the results of 2 subgroups and compared to original rankings of panel 5 . Synthesis of priorities and rankings of alternatives with subgroups compared to overall panel 5 results are presented in Table 40 below.

\begin{tabular}{|l|c|c|c|}
\hline \multicolumn{1}{|c|}{ Alternatives } & Original panel 5 & Subgroup "A" & Subgroup "B" \\
\hline Standard Processes for Reimbursement & 1 & 1 & 1 \\
\hline Multi-State License for Telemedicine & 2 & 2 & 2 \\
\hline Continues Training & 3 & 3 & 3 \\
\hline Education & 4 & 4 & 4 \\
\hline Government Support & 5 & 5 & 5 \\
\hline Develop Guidelines for the use of Telemedicine & 6 & 6 & 6 \\
\hline Multi-State Standards and Regulation & 7 & 7 & 7 \\
\hline
\end{tabular}

Table 40 : Ranking of alternative solutions per subgroups in panel 5

The ranking of the alternative solutions remains unchanged with every subgroup compared to the complete panel 5 results. Standard processes for reimbursement still have the highest in importance ranking, followed by multi-state license for telemedicine, continues training, and education. 


\subsubsection{Analysis of results with respect to expert disagreements in panel 6}

Experts in panel 6 disagree on the relative importance of subcriteria under the cultural perspective. Hierarchical clustering analysis distinguished two subgroups in expert panel 6 which lowered the disagreement values. Table 41 below shows the relative importance of subcriteria per subgroups in expert panel 6.

\begin{tabular}{|c|c|c|c|}
\hline Experts & Physician acceptance & Patient acceptance & Patient satisfaction \\
\hline Original panel 6 & 0.524 & 0.289 & 0.186 \\
\hline Subgroup "A" & 0.38 & 0.410 & 0.205 \\
\hline Subgroup "B" & 0.717 & 0.120 & 0.167 \\
\hline
\end{tabular}

Table 41: Relative importance of subcriteria per subgroups in panel 6

The rankings of alternatives were recalculated according to the results of 2 subgroups and compared to original rankings of panel 6 . Synthesis of priorities and rankings of alternatives with subgroups compared to overall panel 6 results are presented in Table 42 below.

\begin{tabular}{|l|c|c|c|}
\hline \multicolumn{1}{|c|}{ Alternatives } & Original panel 6 & Subgroup "A" & Subgroup "B" \\
\hline Standard Processes for Reimbursement & 1 & 1 & 1 \\
\hline Multi-State License for Telemedicine & 2 & 2 & 2 \\
\hline Continues Training & 3 & 3 & 3 \\
\hline Education & 4 & 4 & 4 \\
\hline Government Support & 5 & 5 & 5 \\
\hline Develop Guidelines for the use of Telemedicine & 6 & 6 & 6 \\
\hline Multi-State Standards and Regulation & 7 & 7 & 7 \\
\hline
\end{tabular}

Table 42 : Rankings of alternative solutions per subgroups in panel 6

The ranking of the alternative solutions remains unchanged with every subgroup compared to the complete panel 6 results. Standard processes for 
reimbursement still have the highest in importance ranking, followed by multi-state license for telemedicine, continues training and education.

\subsection{Sensitivity Analysis using scenarios approach}

The Scenario analysis was conducted to understand the effects of changing the relative importance of the main barriers' perspectives on rankings of model alternatives. Extreme weight variations were considered in "what-if" scenarios on perspectives while rankings of the model alternatives were observed. Since there are four main perspectives, four scenarios will be considered in this sensitivity analysis. In each case, one of the perspectives will be assigned a value of 0.97 , while three others will be assigned a value of 0.01 each as shown in Table 43 below $[163,165]$.

\begin{tabular}{|c|c|c|c|c|}
\hline Perspectives & Financial Barriers & Technical Barriers & Logistical Barriers & Cultural Barriers \\
\hline Orginal value & 0.42 & 0.21 & 0.17 & 0.19 \\
\hline Scenario 1 & 0.97 & 0.10 & 0.10 & 0.10 \\
\hline Scenario 2 & 0.10 & 0.97 & 0.10 & 0.10 \\
\hline Scenario 3 & $\mathbf{0 . 1 0}$ & $\mathbf{0 . 1 0}$ & 0.97 & 0.10 \\
\hline Scenario 4 & $\mathbf{0 . 1 0}$ & $\mathbf{0 . 1 0}$ & $\mathbf{0 . 1 0}$ & 0.97 \\
\hline
\end{tabular}

Table 43: Distribution of weights for model perspectives in four scenarios

The scenarios might be useful if the decision makers want to focus only on one of the model perspectives and look at the rankings of the alternatives under that main consideration. Each scenario replicates a situation where there is a different emphasis on one perspective driving the adoption of telemedicine as shown in Table 44 below. 


\begin{tabular}{|c|l|}
\hline Scenario & \multicolumn{1}{c|}{ Description } \\
\hline Financial focus & $\begin{array}{l}\text { The main point of focus in the adoption of telemedicine is financial } \\
\text { barriers which include start-up cost and ongoing cost. }\end{array}$ \\
\hline Technical focus & $\begin{array}{l}\text { The main point of focus in the adoption of telemedicine is technical } \\
\text { barriers which include computer skills, training, technical support, } \\
\text { infrastructure, and privacy \& security. }\end{array}$ \\
\hline Logistical focus & $\begin{array}{l}\text { The main point of focus in the adoption of telemedicine is logistical } \\
\text { barriers which include licensure, credentialing, malpractice, and } \\
\text { reimbursement. }\end{array}$ \\
\hline Cultural focus & $\begin{array}{l}\text { The main point of focus in the adoption of telemedicine is the cultural } \\
\text { barrier which includes physician acceptance, patient acceptance, and } \\
\text { patient satisfaction. }\end{array}$ \\
\hline
\end{tabular}

Table 44 : Description of the four scenarios focus.

\subsubsection{Scenario 1: Financial barriers focus}

In this scenario, the financial barriers were assigned as important criteria with a value of 0.97 and the others with a value of 0.01 each. The result shown in Figure 34 below.

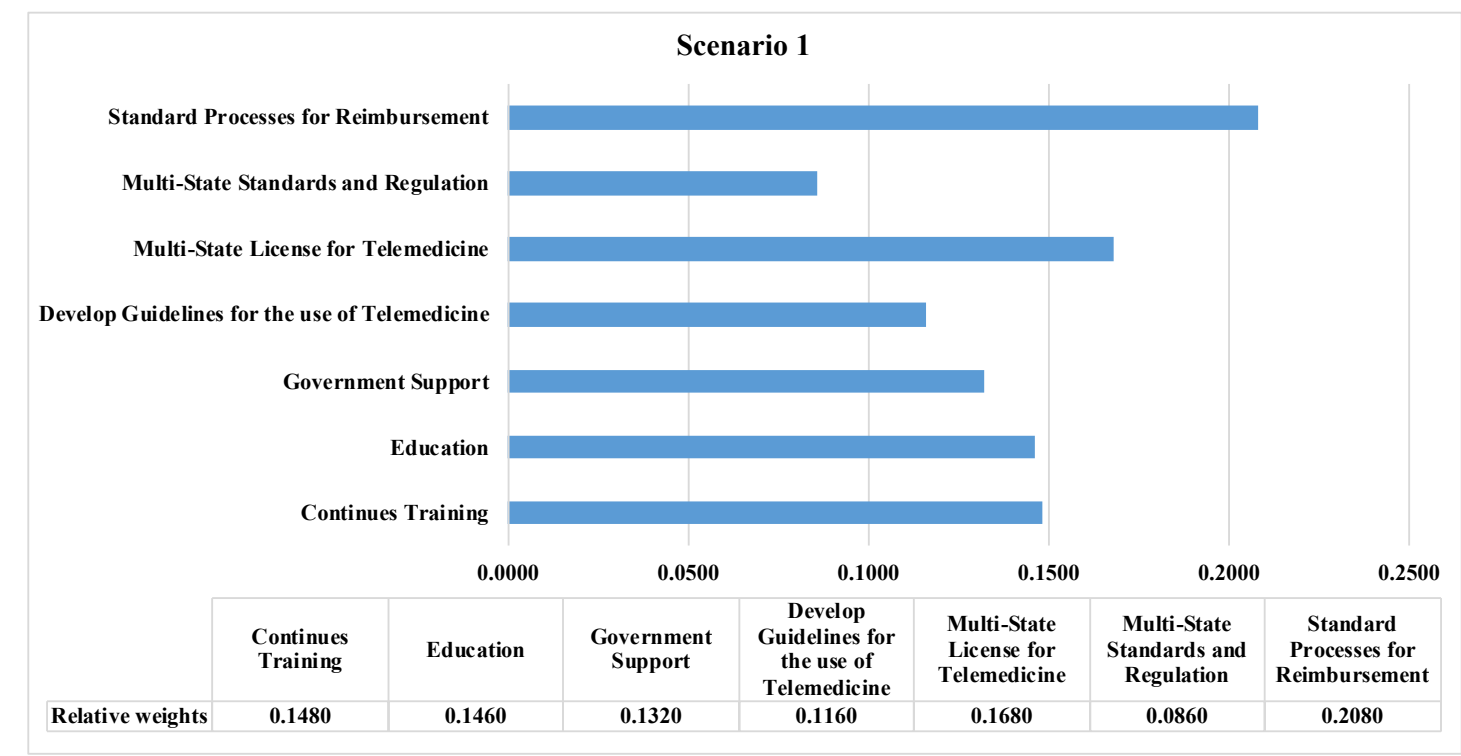

Figure 34 : The global contribution of alternatives to the mission in scenario 1 
According to the analysis above, standard processes for reimbursement (0.208) has the highest importance ranking in scenario 1. Multi-state license for telemedicine takes a second place in the adoption according to financial focus scenario with the value of $(0.1680)$. Multi-state standards and regulation takes the seventh and the last place in the adoption according to financial focus scenario with the value of $(0.0860)$. The alternatives ranking did not change and all alternatives under the financial barriers focus have the same ranking order as the original ranking as shown in Table 45 below.

\begin{tabular}{|l|c|c|c|c|}
\hline \multicolumn{1}{|c|}{ Alternatives } & Base Value & Original Rank & Sensitivity Value & New Rank \\
\hline Continues Training & 0.1470 & 3 & 0.1480 & 3 \\
\hline Education & 0.1450 & 4 & 0.1460 & 4 \\
\hline Government Support & 0.1311 & 5 & 0.1320 & 5 \\
\hline Develop Guidelines for the use of Telemedicine & 0.1152 & 6 & 0.1160 & 6 \\
\hline Multi-State License for Telemedicine & $\mathbf{0 . 1 6 6 9}$ & 2 & $\mathbf{0 . 1 6 8 0}$ & 2 \\
\hline Multi-State Standards and Regulation & $\mathbf{0 . 0 8 5 4}$ & 7 & $\mathbf{0 . 0 8 6 0}$ & 7 \\
\hline Standard Processes for Reimbursement & $\mathbf{0 . 2 0 6 6}$ & 1 & $\mathbf{0 . 2 0 8 0}$ & 1 \\
\hline
\end{tabular}

Table 45 : Scenario 1 overall importance of alternatives

\subsubsection{Scenario 2: Technical barriers focus}

In this scenario, the technical barriers were assigned as important criteria with a value of 0.97 and the others with a value of 0.01 each. The result shown in Figure 35 below. 


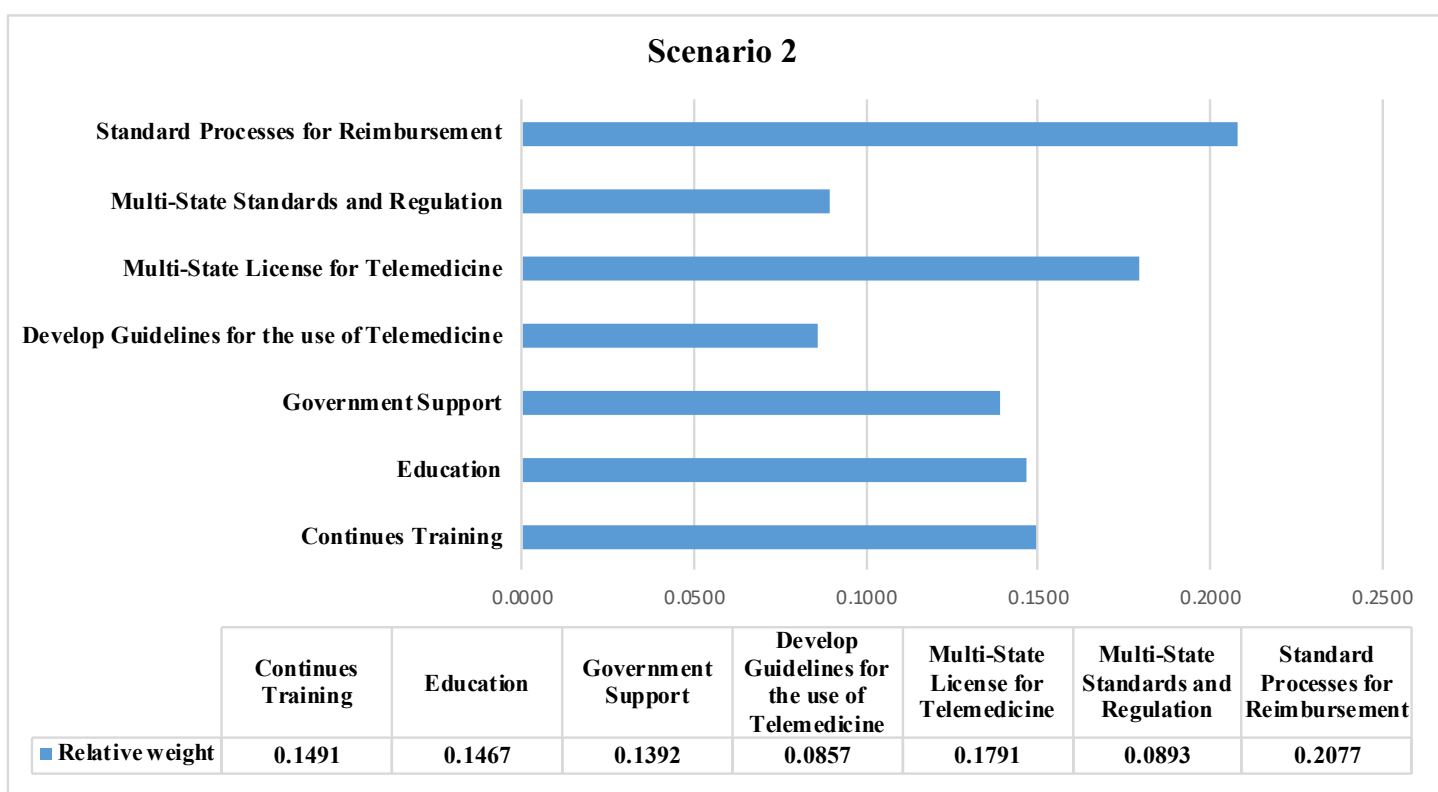

Figure 35 : The global contribution of alternatives to the mission in scenario 2

According to the analysis above, standard processes for reimbursement (0.2077) has the highest importance ranking in scenario 2. Multi-state license for telemedicine takes a second place in the adoption according to technical focus scenario with the value of $(0.1791)$. Multi-state standards and regulation takes the seventh and the last place in the adoption according to technical focus scenario with the value of $(0.0893)$. The alternatives ranking did not change and all alternatives under technical barriers focus have the same ranking order as the original ranking as shown in Table 46 below.

\begin{tabular}{|l|c|c|c|c|}
\hline \multicolumn{1}{|c|}{ Alternatives } & Base Value & Original Rank & Sensitivity Value & New Rank \\
\hline Continues Training & $\mathbf{0 . 1 4 7 0}$ & 3 & 0.1491 & 3 \\
\hline Education & $\mathbf{0 . 1 4 5 0}$ & 4 & 0.1467 & 4 \\
\hline Government Support & $\mathbf{0 . 1 3 1 1}$ & 5 & 0.1392 & 5 \\
\hline Develop Guidelines for the use of Telemedicine & 0.1152 & 6 & 0.0857 & 6 \\
\hline Multi-State License for Telemedicine & $\mathbf{0 . 1 6 6 9}$ & 2 & 0.1791 & 2 \\
\hline Multi-State Standards and Regulation & $\mathbf{0 . 0 8 5 4}$ & 7 & $\mathbf{0 . 0 8 9 3}$ & 7 \\
\hline Standard Processes for Reimbursement & $\mathbf{0 . 2 0 6 6}$ & 1 & $\mathbf{0 . 2 0 7 7}$ & 1 \\
\hline
\end{tabular}

Table 46 : Scenario 2 overall importance of alternatives 


\subsubsection{Scenario 3: Logistical barriers focus}

In this scenario, the logistical barriers were assigned as important criteria with a value of 0.97 and the others with a value of 0.01 each. The result shown in Figure 36 below.

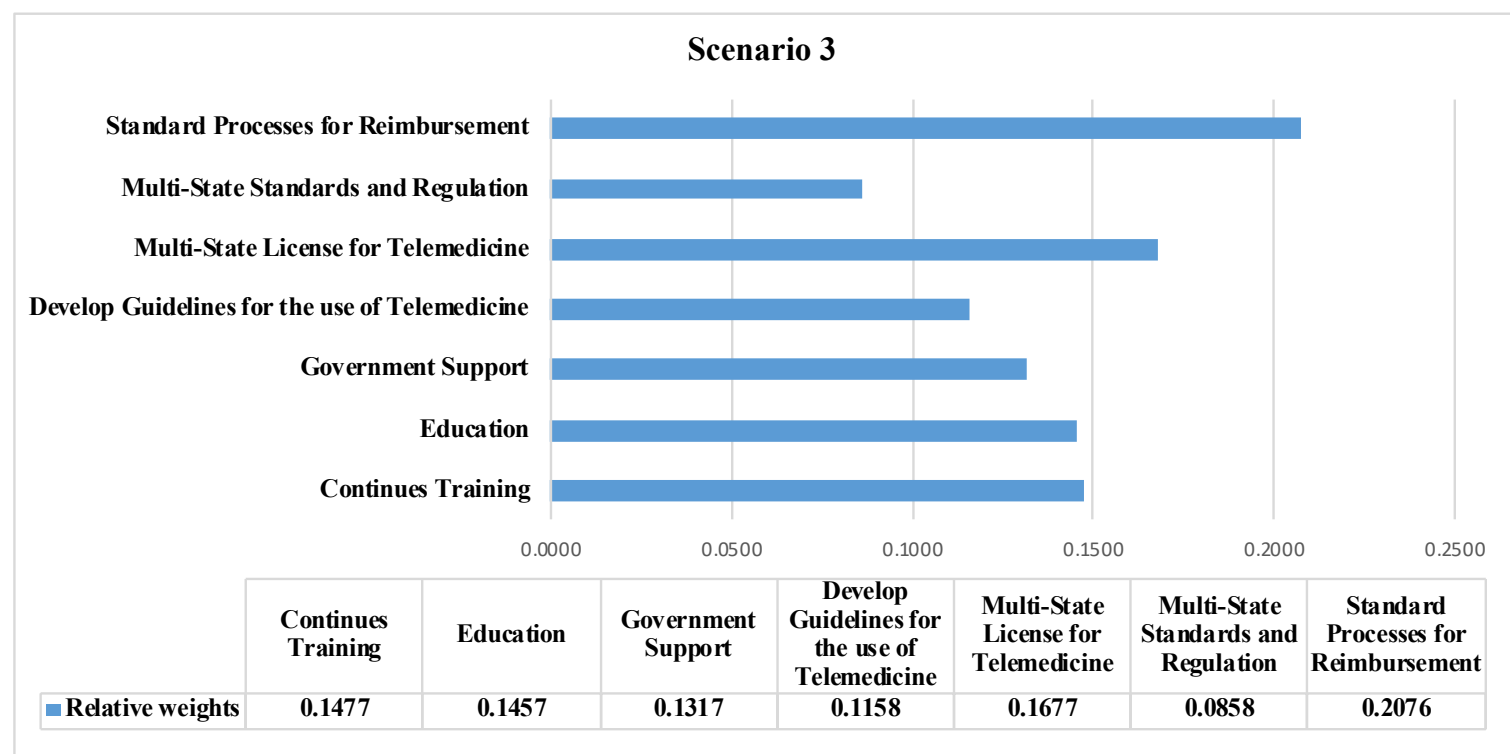

Figure 36 : The global contribution of alternatives to the mission in scenario 3

According to the analysis above, standard processes for reimbursement (0.2076) has the highest importance ranking in scenario 3. Multi-state license for telemedicine takes a second place in the adoption according to logistical focus scenario with the value of $(0.1677)$. Multi-state standards and regulation takes the seventh and the last place in the adoption according to logistical focus scenario with the value of $(0.0858)$. The alternatives ranking did not change and all alternatives under logistical barriers focus have the same ranking order as the original ranking as shown in Table 47 below. 


\begin{tabular}{|l|c|c|c|c|}
\hline \multicolumn{1}{|c|}{ Alternatives } & Base Value & Original Rank & Sensitivity Value & New Rank \\
\hline Continues Training & $\mathbf{0 . 1 4 7 0}$ & 3 & 0.1477 & 3 \\
\hline Education & $\mathbf{0 . 1 4 5 0}$ & 4 & 0.1457 & 4 \\
\hline Government Support & $\mathbf{0 . 1 3 1 1}$ & 5 & 0.1317 & 5 \\
\hline Develop Guidelines for the use of Telemedicine & $\mathbf{0 . 1 1 5 2}$ & 6 & 0.1158 & 6 \\
\hline Multi-State License for Telemedicine & $\mathbf{0 . 1 6 6 9}$ & 2 & $\mathbf{0 . 1 6 7 7}$ & 2 \\
\hline Multi-State Standards and Regulation & $\mathbf{0 . 0 8 5 4}$ & 7 & $\mathbf{0 . 0 8 5 8}$ & 7 \\
\hline Standard Processes for Reimbursement & $\mathbf{0 . 2 0 6 6}$ & 1 & $\mathbf{0 . 2 0 7 6}$ & 1 \\
\hline
\end{tabular}

Table 47: Scenario 3 overall importance of alternatives

\subsubsection{Scenario 4: Cultural barriers focus}

In this scenario, the cultural barriers were assigned as important criteria with a value of 0.97 and the others with a value of 0.01 each. The result shown in Figure

\section{7 below.}

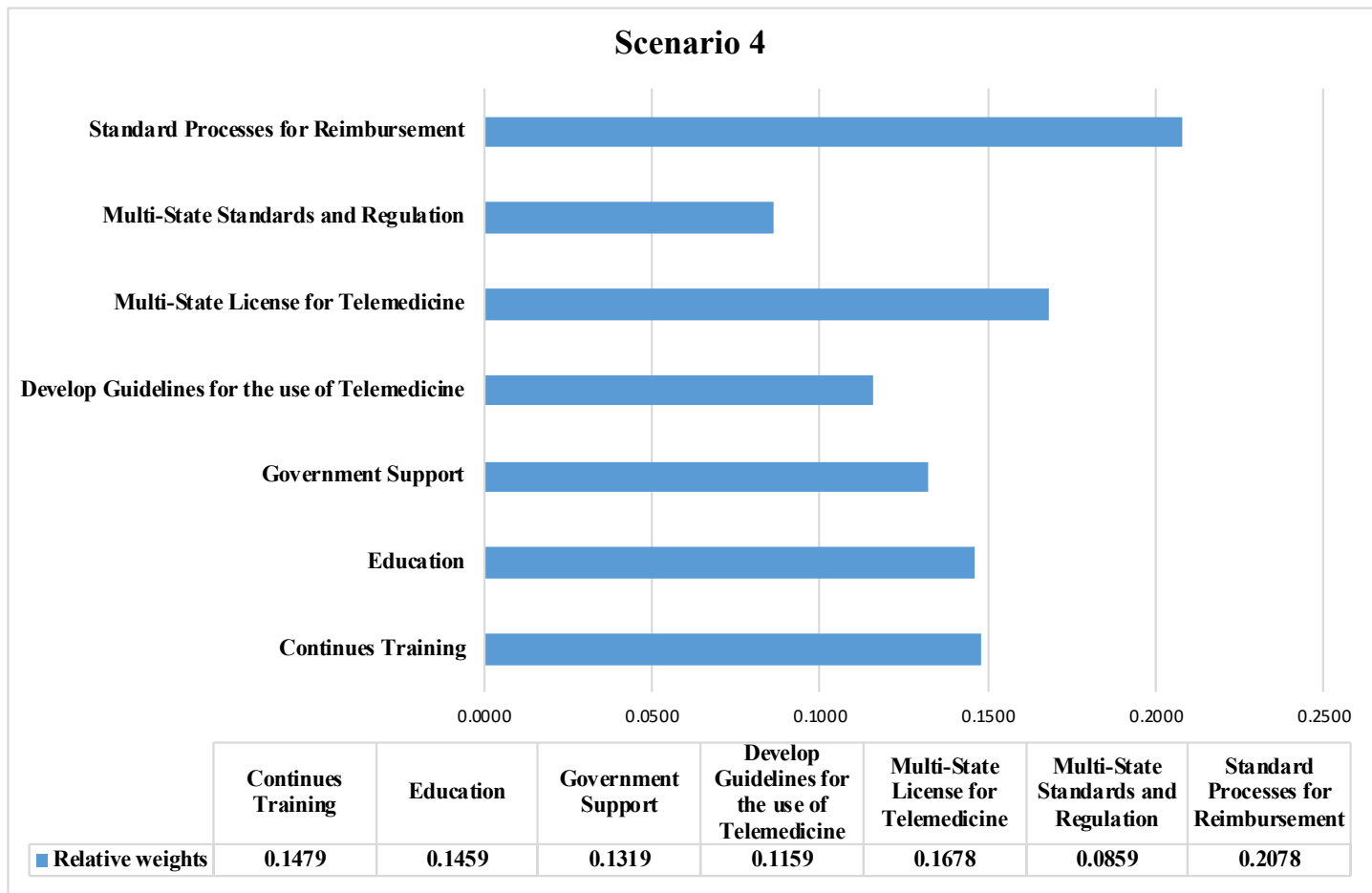

Figure 37 : The global contribution of alternatives to the mission in scenario 4 
According to the analysis above, standard processes for reimbursement (0.2078) has the highest importance ranking in scenario 4. Multi-state license for telemedicine takes a second place in the adoption according to cultural focus scenario with the value of (0.1678). Multi-state standards and regulation takes the seventh and the last place in the adoption according to cultural focus scenario with the value of $(0.0859)$. The alternatives ranking did not change and all alternatives under cultural barriers focus have the same ranking order as the original ranking as shown in Table 48 below.

\begin{tabular}{|l|c|c|c|c|}
\hline \multicolumn{1}{|c|}{ Alternatives } & Base Value & Original Rank & Sensitivity Value & New Rank \\
\hline Continues Training & $\mathbf{0 . 1 4 7 0}$ & 3 & $\mathbf{0 . 1 4 7 9}$ & 3 \\
\hline Education & $\mathbf{0 . 1 4 5 0}$ & 4 & $\mathbf{0 . 1 4 5 9}$ & 4 \\
\hline Government Support & $\mathbf{0 . 1 3 1 1}$ & 5 & $\mathbf{0 . 1 3 1 9}$ & 5 \\
\hline Develop Guidelines for the use of Telemedicine & $\mathbf{0 . 1 1 5 2}$ & 6 & $\mathbf{0 . 1 1 5 9}$ & 6 \\
\hline Multi-State License for Telemedicine & $\mathbf{0 . 1 6 6 9}$ & 2 & $\mathbf{0 . 1 6 7 8}$ & 2 \\
\hline Multi-State Standards and Regulation & $\mathbf{0 . 0 8 5 4}$ & 7 & $\mathbf{0 . 0 8 5 9}$ & 7 \\
\hline Standard Processes for Reimbursement & $\mathbf{0 . 2 0 6 6}$ & 1 & $\mathbf{0 . 2 0 7 8}$ & 1 \\
\hline
\end{tabular}

Table 48 : Scenario 4 overall importance of alternatives

\subsection{Criterion-Related Validity}

Criterion-related validity reflects the degree to which the assessment model is effective in performing in real-life evaluation: meaning that the results and recommendations achieved from the model are applicable, accurate, and valid. Experts were presented with the results of the research and asked to evaluate the appropriateness of the results obtained by the study and the appropriateness of the generalizability of the model. Experts confirmed that the results from the model were appropriate and valid. 


\section{CHAPTER 6: DISCUSSION}

This chapter discusses the results obtained from expert evaluations and the insights that those evaluations provide. Also, this chapter presents recommendations derived from expert feedback received.

\subsection{Technology Acceptance Model (TAM)}

In general, the structure of the technology in healthcare is very complex and we can see that very clear in telemedicine technology. The adopting model shows that the two components of Technology Acceptance Model (TAM): perceived ease of use $(54.4 \%)$ and perceived usefulness $(45.6 \%)$ almost have the same importance with more emphasis on perceived ease of use. The result reflects that the telemedicine technology must be easy to use in all healthcare levels before thinking about its usefulness. The research results matching several studies regarding TAM and telemedicine. Yung-Zung et al. 2015, did research about understanding the determinants of implementing telehealth systems and they found that perceived ease of use positively affects perceived usefulness [166].

However, most of the studies focus only on physician acceptance of telemedicine technology and ignores the other healthcare adoption factors. Paul et al. 1998, did research about examining TAM using physician acceptance of telemedicine technology, and their results concluded that perceived usefulness is a significant determinant of attitude and intention but perceived ease of use was not [167]. Their result does not match our research findings and the reason behind this difference is due to their research focus which is only on the physicians' point of view, where our research looked to the adoption from all perspectives. 


\subsection{Main perspective adoption barriers}

The financial barriers $(38.7 \%)$ ranked the top adoption criteria, technical barriers $(22.6 \%)$, cultural barriers $(20.9 \%)$, and logistical barriers $(17.4 \%)$ almost have the same importance. It is very clear how financial barriers limit the adoption of telemedicine and there are many studies that came up with the same conclusion. A recent study done by Australian researchers finds that funding for telemedicine is frequently mentioned both in Australia and internationally and that includes the lack of funding for doctors, specialists, and their staff to conduct telemedicine consultations [168]. Doctors and hospitals are not going to have much interest in providing telemedicine services or buying the equipment needed to deliver them if they are not confident that they will get paid to offset those costs. Only a few states have passed laws around private payer reimbursement for telemedicine and that varies from state to state. Government payers such as Medicare and Medicaid have their own set of limitations around telemedicine reimbursement.

In the financial barriers, startup costs $(55.3 \%)$ has more importance than ongoing costs (44.7\%). In general, the startup costs are very high in the healthcare industry and for that reason, high startup costs have presented a barrier to many healthcare facilities. There are many healthcare facilities that delay the adoption of telemedicine technology because the cost of implementing new technology often goes far beyond the price of the hardware or service [169]. There are other costs such as licensing and subscription fee, implementation fee, equipment costs, 
installation costs, staff training, technical support, marketing materials, ongoing maintenance, upgrades, and training [170].

The technical barriers (21\%) prevailed as a major issue in successfully implementing telemedicine. The technical barriers include everything that is related to the technical issue involved in the implementation of telemedicine such as computer skills, training, technical support, and infrastructure. Nearly all telemedicine programs depend on high rates of data transmission, so access to broadband Internet is necessary. Specialized application software, data storage devices, database management software, and medical devices capable of electronic data collection, storage, and transmission are all key components of the telemedicine infrastructure [105]. Also, sufficient computer skills required for applying telemedicine, where 'sufficient' means to be comfortable working within the healthcare environment and with healthcare technology in order to enable all staff members to participate in the telemedicine network [103]. It depends on the reliability and professional use of technology to ensure the delivery of a reliable telemedicine service. Training and technical support is also needed for using the system effectively and efficiently [104]. Technical support must be available 24 hours daily to ensure patient safety and it is key to ensuring telemedicine systems sustainability.

Cultural barriers $(18.6 \%)$ are very important barriers to the adoption of a telemedicine system and it depends largely on acceptance by healthcare providers (physicians, nurse practitioners, and physician assistants) [113]. We should acknowledge that user acceptance is important, but physicians' acceptance is 
critical because they are the most important users of telemedicine technology and their acceptance and satisfaction play a crucial role in the further acceptance of telemedicine $[115,171]$. Physicians' acceptance includes everything related to their discomfort in use of the new technology equipment in their daily practices and treating patients at a distance [116].

The logistical barriers (17.4\%) are the least important adoption barriers of telemedicine. One of the main advantages of telemedicine is allowing specialists to help more patients over states. However, physicians face many telemedicine challenges if they wish to practice in another state, including licensing and regulations that vary state-by-state. In some cases, practitioners may need a full medical license in both the state they live in and the state where the patient resides. In other situations, physicians may have to pay an expensive fee to practice across state lines. Additionally, even after obtaining the necessary clearance to practice medicine across state lines, physicians may be asked to follow state medical practice rules that are diverse and even sometimes conflicting [107]. The difficult nature of these laws limits the quick spread of telemedicine.

\subsection{Subcriteria adoption barriers}

The global weight for all sub-criteria is summarized in Table 49 below. Three categories were identified; high category $(\geq 10 \%)$, medium category $(5-9 \%)$ and low category $(1-4 \%)$. 


\begin{tabular}{|c|c|c|c|}
\hline & Sub-criteria & Global weight & Rank \\
\hline \multirow{3}{*}{ 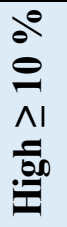 } & Start-up Costs & 0.23 & 1 \\
\hline & Ongoing Cost & 0.19 & 2 \\
\hline & Physician Acceptance & 0.10 & 3 \\
\hline \multirow{4}{*}{ 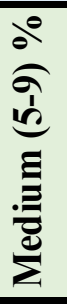 } & Reimbursement & 0.08 & 4 \\
\hline & Privacy \& Security & 0.06 & 5 \\
\hline & Patient Acceptance & 0.05 & 6 \\
\hline & Malpractice & 0.05 & 6 \\
\hline \multirow{7}{*}{ 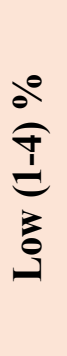 } & Technical Support & 0.04 & 7 \\
\hline & Training & 0.04 & 7 \\
\hline & Patient Satisfaction & $\mathbf{0 . 0 3}$ & 8 \\
\hline & Computer Skills & $\mathbf{0 . 0 3}$ & 8 \\
\hline & Infrastructure & $\mathbf{0 . 0 3}$ & 8 \\
\hline & Licensure & $\mathbf{0 . 0 3}$ & 8 \\
\hline & Credentialing & 0.02 & 9 \\
\hline
\end{tabular}

Table 49: The global weight for the subcriteria

The high category consists of 3 subcriteria startup costs $(23 \%)$, ongoing cost $(19 \%)$, and physician acceptance $(10 \%)$. It is noticeable that the top 2 subcriteria are under the financial barriers which match the weight of the main barrier where the financial barriers $(42.4 \%)$ ranked the top main barriers. This reflects the importance of financial barriers on the adoption of telemedicine applications and must be given more consideration before and during the adoption process.

The medium category consists of 4 subcriteria reimbursement $(8 \%)$, privacy and security $(6 \%)$, patient acceptance $(5 \%)$, and malpractice $(5 \%)$. Two of the medium category subcriteria under logistical barriers (17.4\%) that ranked the least important of the main barrier and that makes possible to distinguish between the 
lower 3 barriers since they have almost the same weight: technical barriers $(21 \%)$, cultural barriers (18.6\%), and logistical barriers (17.4\%).

The low category consists of 7 subcriteria technical support (4\%), training $(4 \%)$, patient satisfaction (3\%), computer skills $(3 \%)$, infrastructure $(3 \%)$, licensure $(3 \%)$, and credentialing (2\%). More than $50 \%$ of the low category under the technical barriers $(21 \%)$ ranked the second important of the main barrier and that one of the advantages of calculating the global weight to differentiate between main barriers.

\subsection{Alternative solutions}

The research identified 7 alternative solutions that can accelerate the adoption of telemedicine application: continues training, education, government support, develop guidelines for the use of telemedicine, multi-state license for telemedicine, multi-state standards and regulation, and standard processes for reimbursement.

According to the experts' input, the standard processes for reimbursement (20.8\%) ranked the top alternatives that can facilitate the adoption following by multi-state license for telemedicine (16.8\%) and continues training (14.8) as shown in Table 50 below. 


\begin{tabular}{|l|c|c|}
\hline \multicolumn{1}{|c|}{ Alternative Solutions } & Percentage & Rank \\
\hline Standard Processes for Reimbursement & $20.80 \%$ & 1 \\
\hline Multi-State License for Telemedicine & $16.80 \%$ & 2 \\
\hline Continues Training & $14.80 \%$ & 3 \\
\hline Education & $14.60 \%$ & 4 \\
\hline Government Support & $13.20 \%$ & 5 \\
\hline Develop Guidelines for the use of Telemedicine & $11.60 \%$ & 6 \\
\hline Multi-State Standards and Regulation & $\mathbf{8 . 6 0 \%}$ & 7 \\
\hline
\end{tabular}

Table 50: The alternative solutions ranking

The government support (13.2\%), develop guidelines for the use of telemedicine $(11.6 \%)$, and multi-state standards and regulation $(8.6 \%)$ are the three least important alternative solutions according to experts' input.

There is a strong relationship between the three levels of the model, the main perspective adoption barriers, subcriteria adoption barriers, and alternative solutions. The top main perspective adoption barriers are financial barriers and the top subcriteria adoption barriers are startup costs. The standard processes for reimbursement are the top alternative solutions which are a financial solution and by emphasizing more in this solution we could reduce the effect of the financial barriers and increase the adoption rate of telemedicine application.

If we look to the alternative solutions from other points of view and try to group them, we can come up with 3 main groups. Group 1, called standard and regulation, includes 3 alternative solutions: develop guidelines for the use of telemedicine, multi-state standards and regulation, and multi-state license for telemedicine. Group 2, called incentives, includes 2 alternative solutions which are standard processes for reimbursement and government support. Group 3, called 
learning, includes 2 alternative solutions which are continues training and education. The 3 groups' weights add together as shown in Figure 38 below.

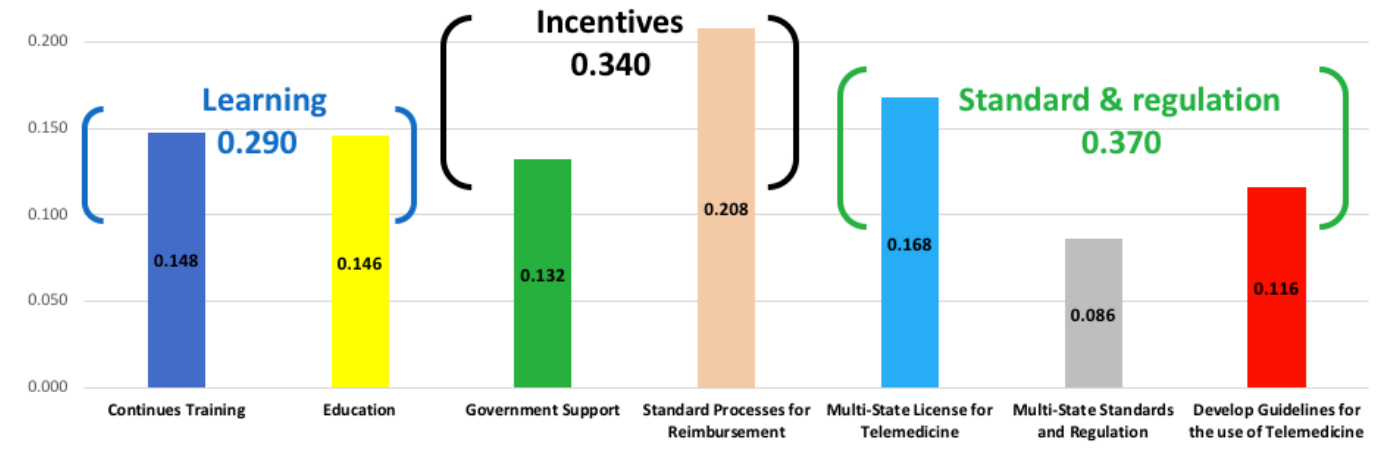

Figure 38: Grouping the alternative solutions

The 3 groups almost have the same importance but group 1, the standard and regulation ranked the top solution with $37.0 \%$ followed by group 2, incentive, with 34.0\%. Group 3, learning, ranked third and the least important solutions with $29.0 \%$.

\subsection{Recommendations}

Telemedicine has revolutionized medicine by increasing access and quality of care while decreasing costs. Telemedicine also reduces the inconveniences, costs, and health effects of traveling to a provider or healthcare facility. To leverage these benefits, many states still need to adapt or expand telemedicine regulations.

\subsubsection{Payment Model}

Grants from organizations and government entities can only do so much to help with the implementation of telemedicine, particularly when those grants are dedicated to specific initiatives or areas. Other types of funding, such as 
reimbursement from government and private payers, must increase in order for this issue to be solved, which should include telemedicine in all payment models, with research to ensure the outcomes and cost-effectiveness of reimbursement levels. Private insurers and the government need to revise their policies around payment to providers for telemedicine services.

\subsubsection{Physician Licensing}

Easing regulations on licensing and geographic areas where telemedicine can be utilized will help telemedicine to reach a broader patient population. One reasonable move that could help solve the problem would be to create a national telemedicine license. The American Telemedicine Association (ATA) has a petition out for removing barriers to medical licensure.

\subsubsection{Privacy and Security}

The success of telemedicine could be undermined if serious privacy and security risks are not addressed. Without adequate security and privacy protections for underlying telemedicine data and systems, providers and patients will lack trust in the use of telemedicine solutions.

Even if the technology markets itself as HIPAA compliant, there still needs to be extra safeguards in place. HIPAA does not have specific telehealth requirements, meaning a telehealth provider must meet the same HIPAA requirements as in-person care. Providers must also abide by any other state privacy laws. Therefore, providers may need to take additional steps, like establishing rules and safeguards for its technical support team, which may be 
inadvertently exposed to patients' protected health information when addressing technical issues. The most detailed information surrounding informed consent for a telehealth session can be found in the ATA guidelines and recommendations that discuss how to maintain privacy and security within the telemedicine session. 


\section{CHAPTER 7: CONCLUSIONS, LIMITATIONS AND FUTURE RESEARCH}

This chapter provides an overview of the conclusion regarding the achievements of the research's objectives, and the research contributions. After collecting all the data and analyzing this data to get the final results, insightful information can be obtained from these analyses. This chapter discusses these conclusions and explains research limitations and future work.

\subsection{Research Conclusions and Contributions}

After collecting all the data and analyzing it, the final results show that the financial barriers ranked the top adoption criteria and it must be given more attention before and during the adoption process in order to minimize their effect. The technical, logistical, and cultural barriers almost have the same effect in the adoption and they must be given the same attention and consideration before and during the adoption process.

Also, the validated HDM gives us the opportunity to know which sub-barriers slow the adoption of teleconsultation technology. After collecting all the data and analyzing this data the final results show that the startup costs, ongoing cost, and physician acceptance respectively are the top 3 subcriteria of the adoption.

The validated HDM gives us the opportunity to know which alternative solutions facilitate more the adoption of teleconsultation technology. After collecting all the data and analyzing this data the final results show that the standard processes for reimbursement ranked the top alternative. Multi-state licenses for teleconsultation, continues training, and education respectively are the second, third, and fourth alternatives that can help the diffusion of teleconsultation. 
Respectively, developing guidelines for the use of teleconsultation and multi-state standards and regulation ranked the least 2 alternatives that can help the diffusion of teleconsultation. The final HDM identified the best alternative solutions to increase the diffusion of teleconsultation. By giving these alternatives more consideration, we could increase the successful adoption rate.

\subsubsection{Theoretical Contributions}

The final research model provided the weight of all criteria, subcriteria, and alternative solutions. Figure 39 below summarize the weight for each element of the research model.

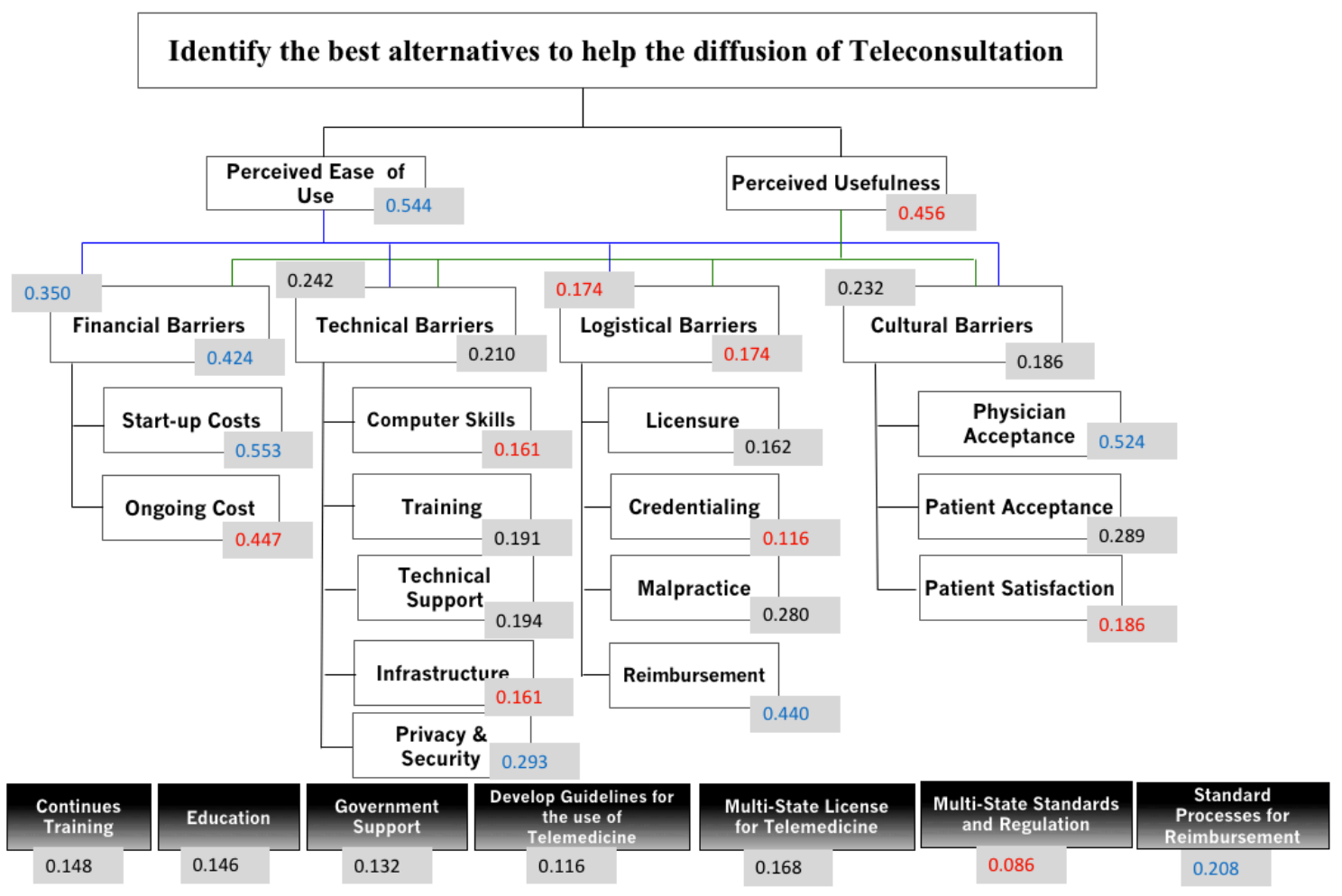

Figure 39: The final model weight 


\subsubsection{Practical Contributions}

The validated HDM model enables healthcare professionals to look at the problem from multiple perspectives and gives us the opportunity to know which criteria and subcriteria matter most in the adoption of teleconsultation technology.

The main contribution of this research is the development of a solid decision making model. Another contribution of this research is the usage of the HDM for healthcare technology assessment problems, in particular the teleconsultation adoption barriers. The research provided insight into how optimum decisions could change in different future scenarios by integrating sensitivity analysis in order to enable decision analysis.

There are many teleconsultation adoption failures, and by applying the final HDM, we can identify the top barriers that cause the failure. By giving these barriers more consideration during the implementation, we can reduce the adoption failure rate. This final HDM can reduce the failure rate of the adoption of teleconsultation by providing early indicators for which criteria or subcriteria needs more attention before and during the implementation or adoption of teleconsultation systems.

The final HDM can increase the success rate of adoption by identifying, from step one, which barriers we could face, and which one could cause the failure. By giving these barriers more attention during or before the adoption of teleconsultation systems, we could likely increase the probability of success of the adoption. 
Overall, initial improvements are expected to contribute to the existing level of knowledge by enabling more alternatives that can provide a better understanding of the adoption barriers and enhance the strategic adoption decisions.

\subsubsection{Policy Contributions}

The final HDM can explain many adoption failures and we come up with some recommendations from these failures. The payment model of telemedicine, private insurers, and the government need to revise their policies around payment to providers for telemedicine services. Include telemedicine in all payment models, with research to ensure the outcomes and cost-effectiveness of reimbursement levels.

Regarding physician licensing, creating a national telemedicine license could help solve the problem. Easing regulations on licensing and geographic areas where telemedicine can be utilized will help to reach a broader patient population.

Regarding privacy and security, providers and patients will lack trust in the use of telemedicine solutions without adequate security and privacy protections for underlying telemedicine data and systems. The ATA guidelines and recommendations must be followed on how to maintain privacy and security within the telemedicine session. 


\subsection{Research Limitation}

This research utilizes the hierarchical decision model methodology. The methodology in this research utilizes the judgments of a number of experts to provide data. Experts in the expert panels are assumed to be capable of providing judgments and have the required knowledge in telemedicine adoption. Expert panels are also assumed to be free of biases. The research consists of several expert panels who validated the results. Since the research engaged multiple expert panels consisting of experts from various fields, academia, engineering, healthcare, and management, and they were asked to validate the results, the results may be impacted by subjectivity. We cannot eliminate expert subjectivity completely, and that is due to the nature of the hierarchical decision model (HDM). However, there are some strategies to increase the reliability of the research. The rigor of expert panels could be increased by avoiding dominant characters and unproductive discussions and equal and straightforward representation of different perspectives.

Research results are also contexed and time-dependent and therefore, could be generalized accordingly. Future possible governmental and insurance reimbursement opportunities could impact the framework of the model. Also, the drivers, impacting adoption decisions, could change for internal or external reasons, which may impact objectives and goals as well as alternatives of the model.

This research also engages various validity measures, like measurements of inconsistency and disagreement. The research approach employs sensitivity 
analyses to gain insight into the impacts of potential scenarios in an attempt to determine the limited degree of generalizability.

The research employed sensitivity analysis that looked at the impacts of potential scenarios according to possible changes in variables and improved the model's generalization ability. Scenario analysis looks at the extreme hypothetical scenarios, where the weight of one perspective is at the maximum, and the rest of the perspectives receive minimum weight contributions. However, other scenarios are possible and should be constructed according to the healthcare department's needs assessing adoption.

The research focuses only on one teleconsultation technology, remote patient monitoring (RPM), and there are many other applications for teleconsultation that can be applied. However, the methodology and the analysis will not change if we change the teleconsultation application.

The research focuses only on one type of patient, elderly patients, and this another limitation of this research. The same research methodology and the analysis could be applied to another segment of patients, such as children or college students.

This research is based on a limited number of experts who identified based on their background and their experiences on this topic. The data collected in this research is based on 44 experts, and this one of the limitations of this research and the increasing number of experts who participate in this research will make the results more reliable and accurate. 
Moreover, the barriers impacting the adoption of teleconsultation technology could change for internal or external reasons. That may have an impact on objectives and goals as well as alternatives to the model. The research employs sensitivity analysis to look at the impacts of potential scenarios according to possible changes in variables and improve the model's generalization ability.

\subsection{Future Research}

Although telemedicine has many definitions, different categories, and classifications, no one can ignore its benefits for patients, physicians, and communities. Telemedicine is still a rich area for valuable innovation and significant investments. The future will bring more opportunities as new technologies improve the quality of telemedicine services, and at the same time realize a huge improvement in telecommunications technology.

The finalized HDM for the adoption barriers for teleconsultation for the senior population gives us the opportunity to learn which criteria and subcriteria matter most in the adoption of telemedicine technology. The model provides a tool for decision makers in telemedicine technology adoption to potentially improve the satisfaction with telemedicine technology in order to achieve the ultimate goal, which is providing high quality healthcare service.

Telemedicine faces many challenges in legislation, regulation, financial, technical, ethical, and cultural challenges, but still, the future is squarely on telemedicine. There is much evidence to support its cost benefits and improve the quality of healthcare services. Telemedicine needs support from individuals, communities, government, healthcare providers, and payers in order to be the first 
option to take over the traditional face-to-face practice. The empirical research and the finalized HDM provide numerous contributions to the body of knowledge.

For future research, we can do an onsite evaluation to get more accurate results by applying the finalized HDM. Further research could be done in another type of telemedicine such as emergency telemedicine, telenursing, teleradiology, telepharmacy, and teleneuropsychology. This research only focuses on senior patients and further research could be done by choosing another segment of patients such as young adults, college students, and children. Also, a similar methodology could be applied for the adoption of any healthcare technology or services. 


\section{REFERENCES}

[1] O. R. Liu Sheng, P. Jen-Hwa Hu, C.-P. Wei, K. Higa, and G. Au, "Adoption and diffusion of telemedicine technology in health care organizations: a comparative case study in Hong Kong," Journal of Organizational Computing and Electronic Commerce, vol. 8, no. 4, pp. 247-275, 1998.

[2] I. Janssen, D. S. Shepard, P. T. Katzmarzyk, and R. Roubenoff, "The healthcare costs of sarcopenia in the United States," Journal of the American Geriatrics Society, vol. 52, no. 1, pp. 80-85, 2004.

[3] P. Zhang et al., "Global healthcare expenditure on diabetes for 2010 and 2030," Diabetes research and clinical practice, vol. 87, no. 3, pp. 293-301, 2010.

[4] P. J.-H. Hu, P. Y. K. Chau, and O. R. L. Sheng, "Investigation of factors affecting healthcare organization's adoption of telemedicine technology," 2000: IEEE, pp. 10-pp.

[5] A. C. Trembly, "Federal Study Supports Telemedicine, But Health Insurers Remain Skeptical.(Brief Article)(Statistical Data Included)," National Underwriter Life \&amp; Health-Financial Services Edition, vol. 105, no. 16, p. 22, 2001.

[6] C. f. M. a. M. S. (CMS), "National Health Expenditures 2013 Highlights," 2013.

[7] G. K. Vincent and V. A. Velkoff, The next four decades: The older population in the United States: 2010 to 2050. US Department of Commerce, Economics and Statistics Administration, US Census Bureau, 2010.

[8] T. M. Dall, P. D. Gallo, R. Chakrabarti, T. West, A. P. Semilla, and M. V. Storm, "An aging population and growing disease burden will require alarge and specialized health care workforce by 2025," Health affairs, vol. 32, no. 11, 2013.

[9] S. L. Colby and J. M. Ortman, "Projections of the Size and Composition of the US Population: 2014 to 2060," US Census Bureau, Ed, pp. 25-1143, 2015.

[10] T. Dall, T. West, R. Chakrabarti, and W. Iacobucci, "The complexities of physician supply and demand: projections from 2013 to 2025," Washington, DC: IHS, 2015.

[11] A. o. A. M. C. (AAMC), "Recent Studies and Reports on Physician Shortages in the US," 2012.

[12] R. S. Hooker, J. F. Cawley, and C. M. Everett, "Predictive modeling the physician assistant supply: 2010-2025," Public Health Reports, pp. 708-716, 2011. 
[13] M. J. Dill and E. S. Salsberg, The complexities of physician supply and demand: projections through 2025. Association of American Medical Colleges, 2008.

[14] P.-Y. Yen and S. Bakken, "Review of health information technology usability study methodologies," Journal of the American Medical Informatics Association, vol. 19, no. 3, pp. 413-422, 2012.

[15] S. Subramanian, S. Hoover, B. Gilman, T. S. Field, R. Mutter, and J. H. Gurwitz, "Computerized physician order entry with clinical decision support in long-term care facilities: costs and benefits to stakeholders," Journal of the American Geriatrics Society, vol. 55, no. 9, pp. 1451-1457, 2007.

[16] M. E. Frisse et al., "The financial impact of health information exchange on emergency department care," Journal of the American Medical Informatics Association, vol. 19, no. 3, pp. 328-333, 2012.

[17] K. S. Reddy et al., "Towards achievement of universal health care in India by 2020: a call to action," The Lancet, vol. 377, no. 9767, pp. 760-768, 2011.

[18] U. E. Reinhardt, P. S. Hussey, and G. F. Anderson, "US health care spending in an international context," Health Affairs, vol. 23, no. 3, pp. 10-25, 2004.

[19] B. L. Brush, J. Sochalski, and A. M. Berger, "Imported care: Recruiting foreign nurses to US health care facilities," Health Affairs, vol. 23, no. 3, pp. 78-87, 2004.

[20] D. A. Dorr and M. M. King, "Health information technology," Comprehensive Care Coordination for Chronically III Adults, pp. 141-165, 2011.

[21] A. M. Audet, D. Squires, and M. M. Doty, "Where are we on the diffusion curve? Trends and drivers of primary care physicians' use of health information technology," Health services research, vol. 49, no. 1pt2, pp. 347-360, 2014.

[22] N. H. Shanks, Introduction to health care management. Jones \& Bartlett Publishers, 2016.

[23] E. Parmelli, G. Flodgren, F. Beyer, N. Baillie, M. E. Schaafsma, and M. P. Eccles, "The effectiveness of strategies to change organisational culture to improve healthcare performance: a systematic review," Implementation Science, vol. 6, no. 1, p. 1, 2011.

[24] P. Pawar, V. Jones, B.-J. F. Van Beijnum, and H. Hermens, "A framework for the comparison of mobile patient monitoring systems," Journal of biomedical informatics, vol. 45, no. 3, pp. 544-556, 2012.

[25] R. Wootton, J. Craig, and V. Patterson, Introduction to telemedicine. Royal Society of Medicine Press, 2006. 
[26] A. T. Association. "What is Telemedicine?"

[27] O. World Health, Telemedicine: opportunities and developments in Member States: report on the second global survey on eHealth. World Health Organization, 2010.

[28] S. H. Schug, C. Flim, and J. Attema, "Sustainable Telemedicine: paradigms for future-proof healthcare-A Briefing Paper," Version, vol. 1, p. 20, 2008.

[29] V. Ramos, "Contributions to the history of TelemediciNe of the TICs," in Telecommunications Conference (HISTELCON), 2010 2010: IEEE, pp. 1-5.

[30] J. Craig and V. Patterson, "Introduction to the practice of telemedicine," Journal of Telemedicine and Telecare, vol. 11, no. 1, pp. 3-9, 2005.

[31] B. Stanberry, "Telemedicine: barriers and opportunities in the 21 st century," Journal of internal medicine, vol. 247, no. 6, pp. 615-628, 2000.

[32] C. L. Wittson, D. C. Affleck, and V. Johnson, "Two-way television in group therapy," Mental hospitals, 1961.

[33] K. T. Bird, "Tele-Diagnosis: A New Community Health Resource," Educ Instr Broadcasting, 1969.

[34] D. S. Puskin, "Opportunities and challenges to telemedicine in rural America," Journal of Medical Systems, vol. 19, no. 1, pp. 59-67, 1995.

[35] M. Fuchs, "Provider attitudes toward STARPAHC: a telemedicine project on the Papago reservation," Medical care, vol. 17, no. 1, pp. 59-68, 1979.

[36] R. Makena and C. C. Hayes, "Flexible usage of space for telemedicine," in IEEE International Conference on Systems, Man, and Cybernetics (SMC), AK, USA, 2011 2011: IEEE, pp. 1134-1139.

[37] T. Takahashi, "The present and future of telemedicine in Japan," International journal of medical informatics, vol. 61, no. 2, pp. 131-137, 2001.

[38] S. Meystre, "The current state of telemonitoring: a comment on the literature," Telemedicine Journal \& e-Health, vol. 11, no. 1, pp. 63-69, 2005.

[39] S. Kumar and J. Marescaux, Telesurgery. Springer Science \& Business Media, 2008.

[40] V. R. Curran, "Tele-education," Journal of telemedicine and telecare, vol. 12, no. 2, pp. 57-63, 2006. 
[41] D. A. Forsberg, "Quality assurance in teleradiology," Telemedicine Journal, vol. 1, no. 2, pp. 107-114, 1995.

[42] L. Baer, P. Cukor, M. A. Jenike, L. Leahy, J. O'Laughlen, and J. T. Coyle, "Pilot studies of telemedicine for patients with obsessive-compulsive disorder," American Journal of Psychiatry, vol. 152, no. 9, pp. 1383-1385, 1995.

[43] D. A. Perednia and A. Allen, "Telemedicine technology and clinical applications," Jama, vol. 273, no. 6, pp. 483-488, 1995.

[44] L. R. Lipson and T. M. Henderson, "State initiatives to promote telemedicine," Telemedicine Journal, vol. 2, no. 2, pp. 109-121, 1996.

[45] C. Kesler and D. Balch, "Development of a telemedicine and distance learning network in rural eastern North Carolina," Journal of telemedicine and telecare, vol. 1, no. 3, pp. 178-182, 1995.

[46] A. Allen and D. Allen, "Telemental Health Services Today," Telemedicine Today, vol. 2, no. 1, pp. 12-24, 1994.

[47] L. B. Hammer, E. Allen, and T. D. Grigsby, "Work-family conflict in dual-earner couples: Within-individual and crossover effects of work and family," Journal of vocational behavior, vol. 50, no. 2, pp. 185-203, 1997.

[48] L. Pantanowitz et al., "American Telemedicine Association clinical guidelines for telepathology," Journal of pathology informatics, vol. 5, no. 1, p. 39, 2014.

[49] "2014 Telemedicine Survey." Foley \& Lardner LLP.

[50] BCC Research."Wearable Electronic Devices Rapidly Gaining Ground in Healthcare Information Technology Industry."

[51] A. Coban, I. F. Ertis, and N. A. Cavdaroglu, "Municipal solid waste management via multi-criteria decision making methods: A case study in Istanbul, Turkey," Journal of cleaner production, vol. 180, pp. 159-167, 2018.

[52] G. Villacreses, G. Gaona, J. Martínez-Gómez, and D. J. Jijón, "Wind farms suitability location using geographical information system (GIS), based on multicriteria decision making (MCDM) methods: The case of continental Ecuador," Renewable energy, vol. 109, pp. 275-286, 2017.

[53] J. M. Sánchez-Lozano, M. S. García-Cascales, and M. T. Lamata, "GIS-based onshore wind farm site selection using Fuzzy Multi-Criteria Decision Making methods. Evaluating the case of Southeastern Spain," Applied Energy, vol. 171, pp. 86-102, 2016. 
[54] S. Senthil, K. Murugananthan, and A. Ramesh, "Analysis and prioritisation of risks in a reverse logistics network using hybrid multi-criteria decision making methods," Journal of Cleaner Production, vol. 179, pp. 716-730, 2018.

[55] M. Tahri, M. Hakdaoui, and M. Maanan, "The evaluation of solar farm locations applying Geographic Information System and Multi-Criteria Decision-Making methods: Case study in southern Morocco," Renewable and Sustainable Energy Reviews, vol. 51, pp. 1354-1362, 2015.

[56] V. Penadés-Plà, T. García-Segura, J. V. Martí, and V. Yepes, "A review of multicriteria decision-making methods applied to the sustainable bridge design," Sustainability, vol. 8, no. 12, p. 1295, 2016.

[57] A. Yildiz and A. Y. Yayla, "Multi-criteria decision-making methods for supplier selection: A literature review," South African Journal of Industrial Engineering, vol. 26, no. 2, pp. 158-177, 2015.

[58] S. Dožić, "Multi-criteria decision making methods: Application in the aviation industry," Journal of Air Transport Management, vol. 79, p. 101683, 2019.

[59] D. F. Kocaoglu, "Hierarchical decision modeling," Engineering Management Program, Portland State University, 1988.

[60] D. F. Kocaoglu, "A participative approach to program evaluation," Engineering Management, IEEE Transactions on, no. 3, pp. 112-118, 1983.

[61] D. F. Kocaoglu and M. Guven Iyigun, "Strategic R\&D program selection and resource allocation with a decision support system application," 1994: IEEE, pp. 225-232.

[62] T. Turan, M. Amer, P. Tibbot, M. Almasri, F. Al Fayez, and S. Graham, "Use of Hierarchal Decision Modeling (HDM) for selection of graduate school for master of science degree program in engineering," 2009: IEEE, pp. 535-549.

[63] P. Gerdsri and D. F. Kocaoglu, "HDM for developing national emerging technology strategy and policy supporting sustainable economy: A case study of nanotechnology for Thailand's agriculture," in Management of Engineering \& Technology, 2008. PICMET 2008. Portland International Conference on, 2008: IEEE, pp. 344-350.

[64] N. J. Sheikh, K. Kim, and D. F. Kocaoglu, "Use of hierarchical decision modeling to select target markets for a new personal healthcare device," Health policy and technology, vol. 5, no. 2, pp. 99-112, 2016.

[65] S. A. Gandhi, V. P. Desai, A. S. Abhyankar, and V. Attar, "Traffic Congestion Control Using Hierarchical Decision Model," 2019: Springer, pp. 98-106. 
[66] S. J. Kim, N. J. Sheikh, and G. Stokes, "Assessment of Arms Import Policies Using a Hierarchical Decision Model and Expert Judgments: Case Study of South Korean Arms Procurement Organizations," International Journal of Innovation and Technology Management, vol. 16, no. 06, p. 1950045, 2019.

[67] H. Hu, D. Yarats, Q. Gong, Y. Tian, and M. Lewis, "Hierarchical decision making by generating and following natural language instructions," 2019, pp. 10025-10034.

[68] D. E. Arzola, J. Bauer, J. Binus, S. Purwar, and A. Wittman, "Education Nonprofit Organization: A Hierarchical Decision Modeling Tool for State Engagement Choice," 2017.

[69] M. Dabab, R. Khalifa, and N. Beltaif, "Evaluating the Selection of Cellular Business Using a Hierarchical Decision Model: The Case of Libya," 2019: IEEE, pp. 1-9.

[70] S. Lee and Y.-J. Son, "A Hierarchical Decision-making Process in Social Networks for Disaster Management," 2018.

[71] R. Carli, M. Dotoli, and R. Pellegrino, "A hierarchical decision-making strategy for the energy management of smart cities," IEEE Transactions on Automation Science and Engineering, vol. 14, no. 2, pp. 505-523, 2016.

[72] T. J. Gordon and J. Glenn, "Interactive scenarios," in Innovative research methodologies in management: Springer, 2018, pp. 31-61.

[73] Y. Liu, Y. Li, and Y. Ding, "East Asian summer rainfall projection and uncertainty under a global warming scenario," International Journal of Climatology, 2020.

[74] V. Villa, N. Paltrinieri, F. Khan, and V. Cozzani, "Towards dynamic risk analysis: A review of the risk assessment approach and its limitations in the chemical process industry," Safety science, vol. 89, pp. 77-93, 2016.

[75] B. Barr, J. Higgerson, and M. Whitehead, "Investigating the impact of the English health inequalities strategy: time trend analysis," bmj, vol. 358, p. j3310, 2017.

[76] V. R. Mallampalli et al., "Methods for translating narrative scenarios into quantitative assessments of land use change," Environmental Modelling \& Software, vol. 82, pp. 7-20, 2016.

[77] M. A. Meyer and J. M. Booker, Eliciting and analyzing expert judgment: a practical guide. SIAM, 2001.

[78] H. L. Dreyfus and S. E. Dreyfus, "Peripheral vision expertise in real world contexts," Organization studies, vol. 26, no. 5, pp. 779-792, 2005. 
[79] K. A. Ericsson, M. J. Prietula, and E. T. Cokely, "The making of an expert," Harvard business review, vol. 85, no. 7/8, p. 114, 2007.

[80] N. Jairath and J. Weinstein, "The Delphi methodology (Part one): A useful administrative approach," Canadian journal of nursing administration, vol. 7, no. 3, pp. 29-42, 1993.

[81] S. M. Millett and E. J. Honton, A manager's guide to technology forecasting and strategy analysis methods. Columbus, Ohio: Battelle Press, 1991, pp. xi, 99 p.

[82] E. R. Babbie, The practice of social research, 6th ed. Belmont, Calif.: Wadsworth Pub. Co., 1992.

[83] N. Black et al., "Consensus development methods: a review of best practice in creating clinical guidelines," Journal of health services research \& policy, vol. 4, no. 4, pp. 236-248, 1999.

[84] R. Cooke and L. Goossens, "Procedures guide for structured expert judgment," Brussels-Luxembourg: Commission of the European Communities, 1999.

[85] K. Q. Hill and J. Fowles, "The methodological worth of the Delphi forecasting technique," Technological Forecasting and Social Change, vol. 7, no. 2, pp. 179$192,1975$.

[86] O. Kuusi, "Expertise in the future use of generic technologies," VATT: $n$ tutkimuksia, vol. 59, 1999.

[87] C. M $\oslash$ ldrup, J. M. Morgall, and A. B. Almarsdóttir, "Perceived risk of future drugs--A Danish citizen Delphi," Health, Risk \& Society, vol. 4, no. 1, pp. 5-17, 2002.

[88] G. Rowe, G. Wright, and F. Bolger, "Delphi: a reevaluation of research and theory," Technological Forecasting and Social Change, vol. 39, no. 3, pp. 235$251,1991$.

[89] D. D. Heckathorn, "Respondent-driven sampling: a new approach to the study of hidden populations," Social problems, vol. 44, no. 2, pp. 174-199, 1997.

[90] D. D. Heckathorn, "Respondent-driven sampling II: deriving valid population estimates from chain-referral samples of hidden populations," Social problems, vol. 49, no. 1, pp. 11-34, 2002.

[91] S. A. Morris and B. Van der Veer Martens, "Mapping research specialties," Annual review of information science and technology, vol. 42, no. 1, pp. 213-295, 2008 
[92] C. Okoli and S. D. Pawlowski, "The Delphi method as a research tool: an example, design considerations and applications," Information \& Management, vol. 42, no. 1, pp. 15-29, 2004.

[93] P. Shah, "Expert Finding Using Social Networking," 2009.

[94] L. C. Leung and D. Cao, "On consistency and ranking of alternatives in fuzzy AHP," European Journal of Operational Research, vol. 124, no. 1, pp. 102-113, 2000.

[95] D. F. Kocaoglu, "A systems approach to the resource allocation process in police patrol," University of Pittsburgh., 1976.

[96] J. L. Mumpower and T. R. Stewart, "Expert judgement and expert disagreement," Thinking \& Reasoning, vol. 2, no. 2-3, pp. 191-212, 1996.

[97] N. Gerdsri and D. F. Kocaoglu, "An analytical approach to building a technology development envelope (TDE) for roadmapping of emerging technologies: a case study of emerging electronic cooling technologies for computer servers," in Management of Engineering and Technology, 2003. PICMET'03. Technology Management for Reshaping the World. Portland International Conference on, 2003: IEEE, pp. 380-389.

[98] J. J. Bartko, "On various intraclass correlation reliability coefficients," Psychological bulletin, vol. 83, no. 5, p. 762, 1976.

[99] H. Chen, Sensitivity analysis for hierarchical decision models. Portland State University, 2007.

[100] H. Chen and D. F. Kocaoglu, "A sensitivity analysis algorithm for hierarchical decision models," European Journal of Operational Research, vol. 185, no. 1, pp. 266-288, 2008.

[101] R. L. Bashshur, T. G. Reardon, and G. W. Shannon, "Telemedicine: a new health care delivery system," Annual Review of Public Health, vol. 21, no. 1, pp. 613$637,2000$.

[102] G. A. Loomis, J. S. Ries, R. M. Saywell, and N. R. Thakker, "If electronic medical records are so great, why aren't family physicians using them?," Journal of Family Practice, vol. 51, no. 7, pp. 636-641, 2002.

[103] D. L. Paul, K. E. Pearlson, and R. R. McDaniel Jr, "Assessing technological barriers to telemedicine: technology-management implications," Engineering Management, IEEE Transactions on, vol. 46, no. 3, pp. 279-288, 1999. 
[104] C. O. Bagayoko, H. Müller, and A. Geissbuhler, "Assessment of Internet-based tele-medicine in Africa (the RAFT project)," Computerized Medical Imaging and Graphics, vol. 30, no. 6, pp. 407-416, 2006.

[105] N. Barry, "Telemedicine diffusion in Scotland: training and technical support issues," British Journal of Healthcare Computing and Information Management, vol. 17, no. 5, pp. 20-23, 2000.

[106] C. E. Lewis, "My Computer, My Doctor: A Constitutional Call for Federal Regulation of Cybermedicine," American journal of law \& medicine, vol. 32, no. 4, pp. 585-609, 2006.

[107] R. C. Ashley, "Telemedicine: Legal, ethical, and liability considerations," Journal of the American Dietetic Association, vol. 102, no. 2, pp. 267-269, 2002.

[108] R. Fitzgerald, "Medical regulation in the telemedicine era," The Lancet, vol. 372, no. 9652, pp. 1795-1796, 2008.

[109] C. Natoli, "Summary of findings: Malpractice and telemedicine," Retrieved Oct, vol. 5, p. 2012, 2009.

[110] B. Chaudhry et al., "Systematic review: impact of health information technology on quality, efficiency, and costs of medical care," Annals of internal medicine, vol. 144, no. 10, pp. 742-752, 2006.

[111] G. Wachter, "HIPAA's Privacy Rule Summarized: What does it mean for Telemedicine," Telemedicine Regulatory Issue Summary. Retrieved September, vol. 13, p. 2003, 2001.

[112] P. A. Clark, K. Capuzzi, and J. Harrison, "Telemedicine: medical, legal and ethical perspectives," American Journal of Case Reports, vol. 16, no. 12, pp. RA261-RA272, 2010.

[113] K. McConnochie, N. Wood, N. Herendeen, C. ten Hoopen, L. Denk, and J. Neuderfer, "Integrating telemedicine in urban pediatric primary care: provider perspectives and performance," TELEMEDICINE and e-HEALTH, vol. 16, no. 3, pp. 280-288, 2010.

[114] R. Latifi, Current principles and practices of telemedicine and e-health. Ios Press, 2008.

[115] K. A. Frey and R. L. Bratton, "Role of telemedicine in the health care delivery system," The Journal of the American Board of Family Practice, vol. 15, no. 2, pp. 170-171, 2002. 
[116] P. Y. K. Chau and P. J.-H. Hu, "Investigating healthcare professionals' decisions to accept telemedicine technology: an empirical test of competing theories," Information \& management, vol. 39, no. 4, pp. 297-311, 2002.

[117] M. P. Yip, A. M. Chang, J. Chan, and A. E. MacKenzie, "Development of the Telemedicine Satisfaction Questionnaire to evaluate patient satisfaction with telemedicine: a preliminary study," Journal of Telemedicine and Telecare, vol. 9, no. 1, pp. 46-50, 2003.

[118] S. S. Gustke, D. C. Balch, V. L. West, and L. O. Rogers, "Patient satisfaction with telemedicine," Telemedicine Journal, vol. 6, no. 1, pp. 5-13, 2000.

[119] I. Ajzen and M. Fishbein, "Understanding attitudes and predicting social behaviour," 1980.

[120] I. Ajzen, From intentions to actions: A theory of planned behavior. Springer, 1985.

[121] I. Ajzen and T. J. Madden, "Prediction of goal-directed behavior: Attitudes, intentions, and perceived behavioral control," Journal of experimental social psychology, vol. 22, no. 5, pp. 453-474, 1986.

[122] M. Fishbein and I. Ajzen, Belief, attitude, intention and behavior: An introduction to theory and research. 1975.

[123] E. Rogers, "M.(1983). Diffusion of innovations," New York, 1983.

[124] L. G. Tornatzky and K. J. Klein, "Innovation characteristics and innovation adoption-implementation: A meta-analysis of findings," Engineering Management, IEEE Transactions on, no. 1, pp. 28-45, 1982.

[125] F. D. Davis, R. P. Bagozzi, and P. R. Warshaw, "User acceptance of computer technology: a comparison of two theoretical models," Management science, vol. 35, no. 8, pp. 982-1003, 1989.

[126] I. Ajzen, "The theory of planned behavior," Organizational behavior and human decision processes, vol. 50, no. 2, pp. 179-211, 1991.

[127] F. D. Davis, "Perceived usefulness, perceived ease of use, and user acceptance of information technology," MIS quarterly, pp. 319-340, 1989.

[128] F. D. Davis, "User acceptance of information technology: system characteristics, user perceptions and behavioral impacts," International journal of man-machine studies, vol. 38, no. 3, pp. 475-487, 1993.

[129] C. Sicotte and P. Lehoux, "Teleconsultation: rejected and emerging uses," Methods Archive, vol. 42, no. 4, pp. 451-457, 2003. 
[130] P. Lehoux, C. Sicotte, J. L. Denis, M. Berg, and A. Lacroix, "The theory of use behind telemedicine:: how compatible with physicians' clinical routines?," Social science \& medicine, vol. 54, no. 6, pp. 889-904, 2002.

[131] M. Nerlich and U. Schaechinger, Integration of health telematics into medical practice. IOS Press, 2003.

[132] N. Khoury, "Doctors Truly Without Borders:

Teleconsultation in the 21st Century," ed. Dubai, UAE: National Arab American Medical Association: NAAMA, 2008.

[133] J. L. Huston and D. C. Burton, "Patient satisfaction with multispecialty interactive teleconsultations," Journal of Telemedicine and Telecare, vol. 3, no. 4, pp. 205208, 1997.

[134] A. W. Darkins and M. A. Cary, Telemedicine and telehealth: principles, policies, performances and pitfalls. Springer publishing company, 2000.

[135] F. Verhoeven, K. Tanja-Dijkstra, N. Nijland, G. Eysenbach, and L. van GemertPijnen, "Asynchronous and synchronous teleconsultation for diabetes care: a systematic literature review," Journal of diabetes science and technology, vol. 4, no. 3, pp. 666-684, 2010.

[136] K. Adambounou et al., "[System of telesonography with synchronous teleconsultations and asynchronous telediagnoses (Togo)]," Medecine et sante tropicales, vol. 22, no. 1, pp. 54-60, 2011.

[137] R. Luk, M. Ho, and P. M. Aoki, "Asynchronous remote medical consultation for Ghana," 2008: ACM, pp. 743-752.

[138] C. M. Barnard and M. E. Goldyne, "Evaluation of an asynchronous teleconsultation system for diagnosis of skin cancer and other skin diseases," Telemedicine Journal and e-Health, vol. 6, no. 4, pp. 379-384, 2000.

[139] P. Rice, "Teleconsultation for Healthcare Services. A workbook for implementing new service models," ed: Yorkshire \& Humber HIEC Bradford, UK, 2011.

[140] A. J. Larner, "Teleneurology: an overview of current status," Practical neurology, vol. 11, no. 5, pp. 283-288, 2011.

[141] E. J. Nordal, D. Moseng, B. Kvammen, and M. L. Løchen, "A comparative study of teleconsultations versus face-to-face consultations," Journal of Telemedicine and Telecare, vol. 7, no. 5, pp. 257-265, 2001.

[142] F. E. Ferrante, "Evolving telemedicine/ehealth technology," Telemedicine Journal \& e-Health, vol. 11, no. 3, pp. 370-383, 2005. 
[143] Y. M. Chae, J. H. Lee, S. H. Ho, H. J. Kim, K. H. Jun, and J. U. Won, "Patient satisfaction with telemedicine in home health services for the elderly," International journal of medical informatics, vol. 61, no. 2, pp. 167-173, 2001.

[144] N. van den Berg, M. Schumann, K. Kraft, and W. Hoffmann, "Telemedicine and telecare for older patients - a systematic review," Maturitas, vol. 73, no. 2, pp. 94-114, 2012.

[145] T. Botsis, G. Demiris, S. Pedersen, and G. Hartvigsen, "Home telecare technologies for the elderly," Journal of Telemedicine and Telecare, vol. 14, no. 7, pp. 333-337, 2008.

[146] M.-k. Suh et al., "A remote patient monitoring system for congestive heart failure," Journal of medical systems, vol. 35, no. 5, pp. 1165-1179, 2011.

[147] S. I. Chaudhry et al., "Telemonitoring in patients with heart failure," New England Journal of Medicine, vol. 363, no. 24, pp. 2301-2309, 2010.

[148] D. Niyato, E. Hossain, and S. Camorlinga, "Remote patient monitoring service using heterogeneous wireless access networks: architecture and optimization," Selected Areas in Communications, IEEE Journal on, vol. 27, no. 4, pp. 412-423, 2009.

[149] K. D. Blanchet, "Remote patient monitoring," Telemedicine and e-Health, vol. 14, no. 2, pp. 127-130, 2008.

[150] H. S. Lin and W. W. Stead, Computational Technology for Effective Health Care:: Immediate Steps and Strategic Directions. National Academies Press, 2009.

[151] M. J. Field and J. Grigsby, "Telemedicine and remote patient monitoring," Jama, vol. 288, no. 4, pp. 423-425, 2002.

[152] R. Pekmezaris et al., "The impact of remote patient monitoring (telehealth) upon Medicare beneficiaries with heart failure," Telemedicine and e-health, vol. 18, no. 2, pp. 101-108, 2012.

[153] A. G. Logan et al., "Mobile Phone-Based Remote Patient Monitoring System for Management of Hypertension in Diabetic Patients*," American journal of hypertension, vol. 20, no. 9, pp. 942-948, 2007.

[154] A. Niksch, B. Rothman, N. Hodge, and M. Ranney, "The Value of Remote Patient Monitoring (RPM) Physicians' Perspectives," Innovation, vol. 401, p. 10.

[155] P. Raj, A. Raman, D. Nagaraj, and S. Duggirala, High-Performance Big-Data Analytics: Computing Systems and Approaches. Springer, 2015. 
[156] S. D. Anker, F. Koehler, and W. T. Abraham, "Telemedicine and remote management of patients with heart failure," The Lancet, vol. 378, no. 9792, pp. 731-739, 2011.

[157] A. Darkins et al., "Care Coordination/Home Telehealth: the systematic implementation of health informatics, home telehealth, and disease management to support the care of veteran patients with chronic conditions," Telemedicine and e-Health, vol. 14, no. 10, pp. 1118-1126, 2008.

[158] B. A. Rosser and C. Eccleston, "Smartphone applications for pain management," Journal of telemedicine and telecare, vol. 17, no. 6, pp. 308-312, 2011.

[159] N. Nakamura, T. Koga, and H. Iseki, "A meta-analysis of remote patient monitoring for chronic heart failure patients," Journal of telemedicine and telecare, vol. 20, no. 1, pp. 11-17, 2014.

[160] Z. D. Gellis, B. Kenaley, J. McGinty, E. Bardelli, J. Davitt, and T. Ten Have, "Outcomes of a telehealth intervention for homebound older adults with heart or chronic respiratory failure: a randomized controlled trial," The Gerontologist, vol. 52, no. 4, pp. 541-552, 2012.

[161] N. Oudshoorn, "How places matter: telecare technologies and the changing spatial dimensions of healthcare," Social studies of science, vol. 42, no. 1, pp. 121-142, 2012.

[162] N. M. Lorenzi and R. T. Riley, Organizational aspects of health informatics: managing technological change. Springer Science \& Business Media, 2013.

[163] I. Iskin, "An Assessment Model for Energy Efficiency Program Planning in Electric Utilities: Case of the Pacific of Northwest U.S.A," Ph.D. Thesis, Portland State University, 2014.

[164] P. Gerdsri and D. Kocaoglu, "A systematic approach to developing national technology policy and strategy for emerging technologies: A case study of nanotechnology for Thailand's agriculture industry," 2009: IEEE, pp. 447-461.

[165] R. Abotah, "Evaluation of Energy Policy Instruments for the Adoption of Renewable Energy: Case of Wind Energy in the Pacific Northwest U.S," Ph.D. Thesis, Portland State University., 2015.

[166] Y.-Z. Chang et al., "Understanding the determinants of implementing telehealth systems: a combined model of the theory of planned behavior and the technology acceptance model," Journal of Applied Sciences, vol. 15, no. 2, p. 277, 2015.

[167] P. J. Hu, P. Y. K. Chau, O. R. L. Sheng, and K. Y. Tam, "Examining the technology acceptance model using physician acceptance of telemedicine 
technology," Journal of management information systems, vol. 16, no. 2, pp. 91$112,1999$.

[168] J. Moffatt and D. S. Eley, "Barriers to the up-take of telemedicine in Australia-a view from providers," 2011.

[169] T. Reardon, "Research findings and strategies for assessing telemedicine costs," Telemedicine Journal \& e-Health, vol. 11, no. 3, pp. 348-369, 2005.

[170] G. Kumar, D. M. Falk, R. S. Bonello, J. M. Kahn, E. Perencevich, and P. Cram, "The costs of critical care telemedicine programs: a systematic review and analysis," Chest, vol. 143, no. 1, pp. 19-29, 2013.

[171] R. Latifi, Current principles and practices of telemedicine and e-health (no. 131). Ios Press, 2008.

[172] Isabalija, Stephen R., et al. "Factors affecting adoption, implementation and sustainability of telemedicine information systems in Uganda." Journal of health informatics in developing countries 5.2 (2011).

[173] Gomes, R., Rossi, R., Lima, S., Carmo, P., Ferreira, R., Menezes, I., ... \& Maciel, P. (2010). Pediatric cardiology and telemedicine: seven years' experience of cooperation with remote hospitals. Revista portuguesa de cardiologia: orgao oficial da Sociedade Portuguesa de Cardiologia $=$ Portuguese journal of cardiology: an official journal of the Portuguese Society of Cardiology, 29(2), 181.

[174] Loane, M., \& Wootton, R. (2002). A review of guidelines and standards for telemedicine. Journal of telemedicine and telecare, 8(2), 63-71.

[175] LeRouge, C., \& Garfield, M. J. (2013). Crossing the telemedicine chasm: have the US barriers to widespread adoption of telemedicine been significantly reduced? International journal of environmental research and public health, 10(12), 64726484 .

[176] Whitten, P., \& Buis, L. (2007). Private payer reimbursement for telemedicine services in the United States. Telemedicine and e-Health, 13(1), 15-24.

[177] Stanberry, B. (2006). Legal and ethical aspects of telemedicine. Journal of telemedicine and telecare, 12(4), 166-175.

[178] Shershneva, M. B., \& Olson, C. A. (2005). Education through telemedicine networks: setting quality standards. Journal of telemedicine and telecare, 11(3), $127-134$. 


\section{APPENDICES}

\section{Appendix A: Human Subject Agreement}

\section{Online Survey Consent Form}

You are invited to participate in a research study, entitled "Exploring Policies and Strategies for the Diffusion of Tele Consultation for the Care of Senior Population." The study is being conducted by Hamad Alanazi, graduate student from Engineering and Technology Management Department at Portland State University. The study is under the supervision my advisor, Tugrul U. Daim.

The purpose of this research study is to examine which strategies are important for removing barriers against the adoption of Tele Consultation. Your participation in the study will contribute to a better understanding of the different criteria with more knowledge to know which criteria in the model require developing and making more research on it to cover it from different perspective. This project is being conducted in partial fulfillment of the requirements for a $\mathrm{PhD}$ degree under the supervision of Dr. Tugrul U. Daim. You are invited as a potential participant due to your expertise in the area of Tele Consultation due to your qualification and professional experience. You are free to contact the investigator at the above address and phone number to discuss the study. You must be at least 18 years old to participate.

If you agree to participate, the evaluation will take approximately 30 minutes of your time and you will complete an activity about Tele Consultation.

There are no known risks to participate and all the information will be kept in my laptop and I will destroy the information after one year of graduation. There are no costs for participating, nor will you personally benefit from participating. Your name and email address will be collected during the data collection phase for tracking purposes only. Identifying information will be stripped from the final dataset.

Your participation in this study is voluntary. You may decline to answer any question and you have the right to withdraw from participation at any time. Withdrawal will not affect your relationship with Portland State University in any way. If you do not want to participate either simply stop participating or close the browser window. I may send study reminders about participation in the study. If you do not want to receive any more reminders, you may email me at halanazi@pdx.edu.

If you have any questions about the study or need to update your email address, send an email to halanazi@pdx.edu. You may also contact my advisor, Tugrul Daim at ji2td@pdx.edu. 
If you have questions about your rights or are dissatisfied at any time with any part of this study, you can contact the Human Subjects Research Review Committee at hsrrc@pdx.edu, Market Center Building, $6^{\text {th }}$ floor, 1600 SW $4^{\text {th }}$ Ave., Portland OR 97201.

If you agree to participate, click on the following link [HTTP://LINK TO STUDY URL]

Thank you.

Hamad Alanazi

Ph.D. Student

Department of Engineering and Technology Management

Portland State University 


\section{Appendix B: Invitation Letter}

Dear Sir/Madam,

My name is Hamad Alanazi and I am a Ph.D. student from the Engineering and Technology Management department at Portland State University. I am writing to invite you to participate in my research study called Exploring Policies and Strategies for the Diffusion of Tele Consultation for the Care of Senior Population. This research study is being conducted in partial fulfillment of the requirements for a $\mathrm{PhD}$ degree in Engineering and Technology Management at Portland State University.

You're eligible to be in this study because you are an expert from either academia or industry and will have enough experience to provide feedback on the criteria in the model I am researching.

Your participation in my research is important to developing a framework, measurement system, and metric for reaching the best benefit of geothermal energy resources. My research looks at the problem from different perspectives and dimensions with respect to utility objectives and goals.

The initial research model that I developed requires participation of experts who have knowledge and opinions in the topic area of Tele Consultation. Participation in the online survey/evaluation will take approximately 30 minutes to complete. This will help to further construct the model and establish a weight for selecting elements that require further development.

If you decide to participate in this study, you will make judgments on different criteria, using paired comparison between two elements, deciding which element is more important between the two. Remember, this is completely voluntary. You can choose to be in the study or not.

If you'd like to participate or have any questions about the study, please email me at halanazi@pdx.edu.

If you have any concerns or problems about participating in this study or your rights as a research subject, please contact the PSU Office of Research Integrity, 1600 SW 4th Ave., Market Center Building Ste. 620, Portland, OR, 97201; phone (503) 725-2227 or 1 (877) $480-4400$

Thank you very much.

Sincerely,

Hamad Alanazi

PhD Student

Department of Engineering and Technology Management

Portland State University 


\title{
Appendix C: Content Validity Instrument
}

\section{Instrument 1}

\author{
P Portland $\underset{\text { UNIVERITY }}{\text { State }}$
}

literature review reveals that there are several barriers impacting the adoption of teleconsultation through perceived usefulness and ease of use, which are the two components of the Technology Acceptance Model (TAM). Please click here to get more information about the Technology Acceptance Model (TAM).

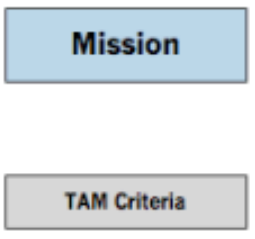

Identify the Best Alternatives to Help the Diffusion of Teleconsultation

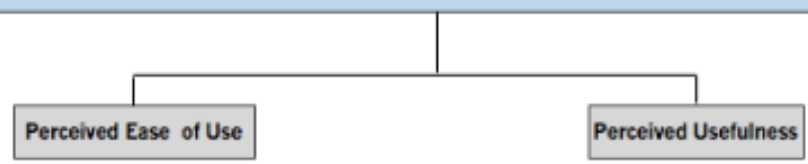

Please indicate whether the diffusion of teleconsultation affected by the two main components of the Technology Acceptance Model (TAM): Perceived Ease of Use and Perceived Usefulness.

\begin{tabular}{l|cc} 
& Yes & No \\
\hline Perceived Ease of Use & $\ddots$ & $\bigcirc$ \\
Perceived Usefulness & $\bigcirc$ & $\bigcirc$
\end{tabular}

Please use the space below for suggesting any additional consideration 


\section{Instrument 2}

面P Portland $\operatorname{UN}_{\text {NIVRSITY }}$

Literature review reveals that there are five main barriers to the adoption of teleconsultation which are financial, technical, logistical, ethical and cultural. Please click here for more information about each barrier.

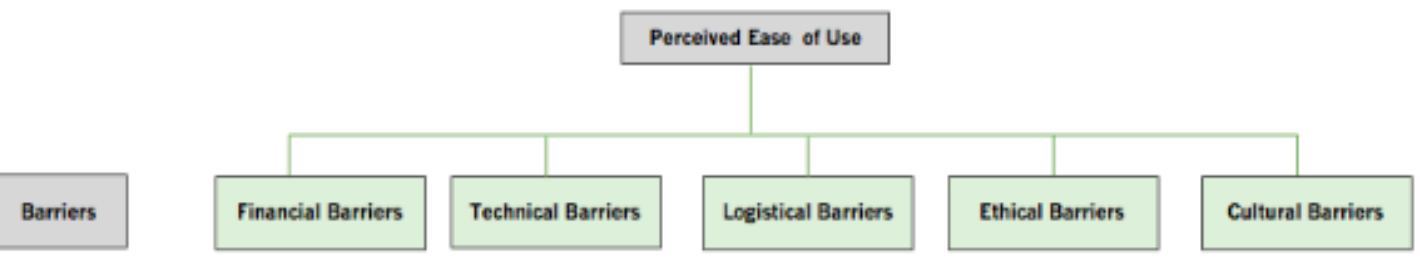

Please indicate whether the proposed barriers below are valid for the assessment of the perceived ease of use.

$\begin{array}{lcc} & \text { Yes } & \text { No } \\ \text { Financial Barriers } & 0 & 0 \\ \text { Technical Barriers } & 0 & 0 \\ \text { Logistical Barriers } & 0 & 0 \\ \text { Ethical Barriers } & 0 & 0 \\ \text { Cultural Barriers } & 0 & 0\end{array}$

. If you think there are other barriers not included in the model, please add them in the space below 
Literature review reveals that there are five main barriers to the adoption of teleconsultation which are financial, technical, logistical, ethical and cultural. Please click here for more information about each barrier.

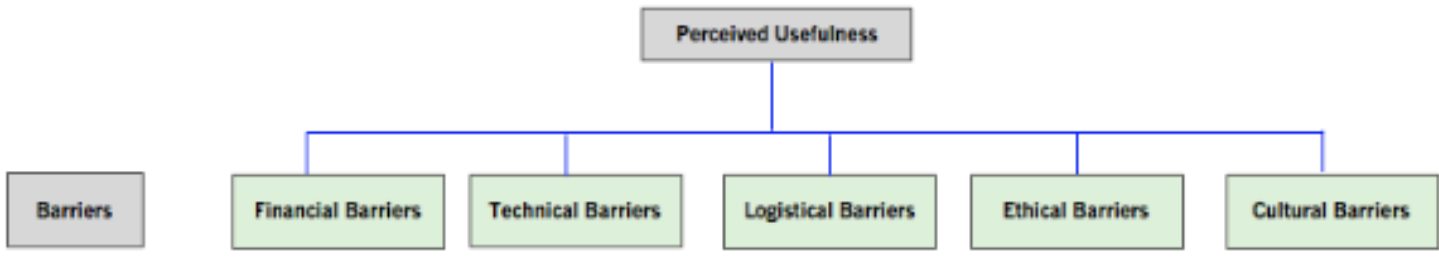

Please indicate whether the proposed barriers below are valid for the assessment of the perceived usefulness.

$\begin{array}{lcr} & \text { Yes } & \text { No } \\ \text { Financial Barriers } & 0 & 0 \\ \text { Technical Barriers } & 0 & 0 \\ \text { Logistical Barriers } & 0 & 0 \\ \text { Ethical Barriers } & 0 & 0 \\ \text { Cultural Barriers } & 0 & 0\end{array}$

. If you think there are other barriers not included in the model, please add them in the space below 


\section{Instrument 3}

P Portland $\underset{\text { UnIVERSITY }}{\text { State }}$

Literature review reveals that there are two sub-barriers associated with the financial barriers to the adoption of teleconsultation. Please click here for more information about each sub-barrier.
Barriers
Financial Barriers
Sub-barriers
Start-up Costs
Ongoing Cost

Please indicate whether the proposed sub-barriers below are valid for the financial barriers of the diffusion of teleconsultation.

\begin{tabular}{l|cc} 
& Yes & No \\
\hline Start-up Cost & 0 & 0 \\
Ongoing Cost & 0 & 0
\end{tabular}

If you think there are other sub-barriers not included in the model, please add them in the space below 


\section{Instrument 4}

面P Portland $\underset{\text { Universitr }}{\text { State }}$

Literature review reveals that there are four sub-barriers associated with the technical barriers to the adoption of teleconsultation. Please click here for more information about each sub-barrier.
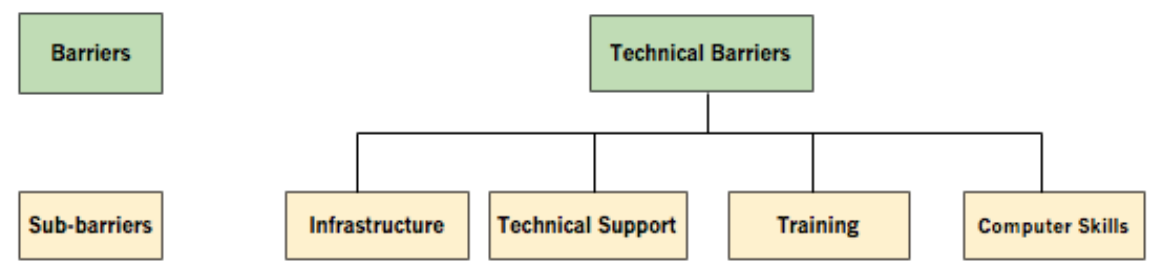

Please indicate whether the proposed sub-barriers below are valid for the technical barriers of the diffusion of teleconsultation.

\begin{tabular}{l|cc} 
& Yes & No \\
\hline Infrastructure & 0 & 0 \\
Technical Support & 0 & 0 \\
Training & 0 & 0 \\
Computer Skills & 0 & 0
\end{tabular}

If you think there are other sub-barriers not included in the model, please add them in the space below 


\section{Instrument 5}

面P Portland $\underset{\text { Universitr }}{\text { State }}$

Literature review reveals that there are four sub-barriers associated with the logistical barriers to the adoption of teleconsultation. Please click here for more information about each sub-barrier.

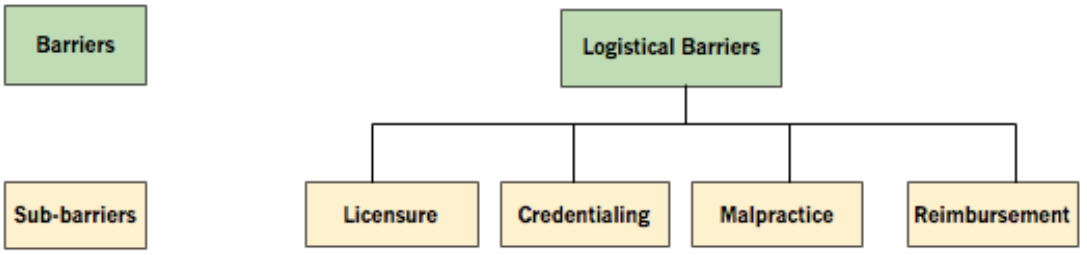

Please indicate whether the proposed sub-barriers below are valid for the logistical barriers of the diffusion of teleconsultation.

\begin{tabular}{l|cc} 
& Yes & No \\
\hline Licensure & 0 & 0 \\
Credentialing & 0 & 0 \\
Malpractice & 0 & 0 \\
Reimbursement & 0 & 0
\end{tabular}

If you think there are other sub-barriers not included in the model, please add them in the space below 


\section{Instrument 6}

面P Portland $\underset{\text { Universitr }}{\text { State }}$

Literature review reveals that there are three sub-barriers associated with the ethical barriers to the adoption of teleconsultation. Please click here for more information about each sub-barrier.
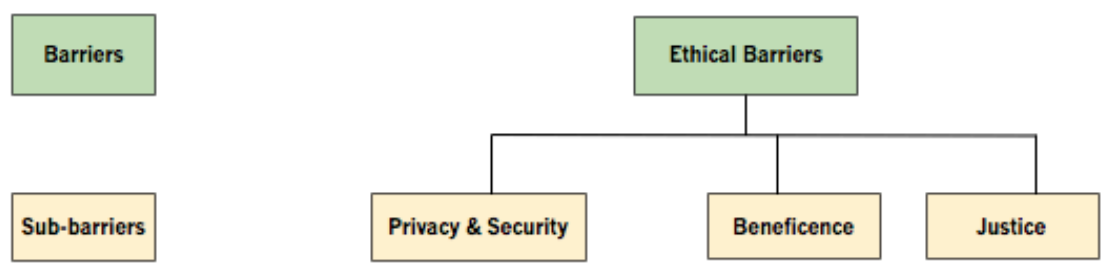

Please indicate whether the proposed sub-barriers below are valid for the ethical barriers of the diffusion of teleconsultation.

\begin{tabular}{l|cc} 
& Yes & No \\
\hline Privacy \& Security & 0 & 0 \\
Beneficence & 0 & 0 \\
Justice & 0 & 0
\end{tabular}

If you think there are other sub-barriers not included in the model, please add them in the space below 


\section{Instrument 7}

面P Portland $\underset{\text { Universitr }}{\text { State }}$

Literature review reveals that there are three sub-barriers associated with the cultural barriers to the adoption of teleconsultation. Please click here for more information about each sub-barrier.

Barriers

Sub-barriers

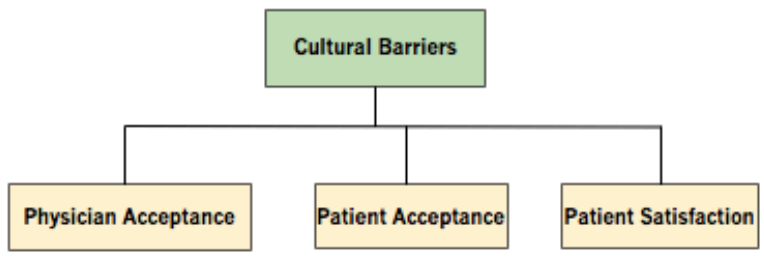

Please indicate whether the proposed sub-barriers below are valid for the cultural barriers of the diffusion of teleconsultation.

\begin{tabular}{l|cc} 
& Yes & No \\
\hline Physician Acceptance & 0 & 0 \\
Patient Acceptance & 0 & 0 \\
Patient Satisfaction & 0 & 0
\end{tabular}

If you think there are other sub-barriers not included in the model, please add them in the space below 


\section{Instrument 8}

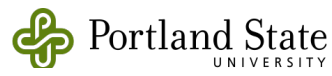

Literature review reveals that there are eight alternative solutions associated with the adoption of teleconsultation. Please click here for more information about each solution.

\section{Continues
Training \\ Education \\ Ethical Code

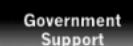 \\ Develop Guidelines for
the use of

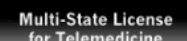

Multi-State Standards

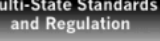

Standard
Processes for
Reimbursement

Please indicate whether the proposed alternative solutions below are valid to address adoption barriers for the diffusion of teleconsultation.

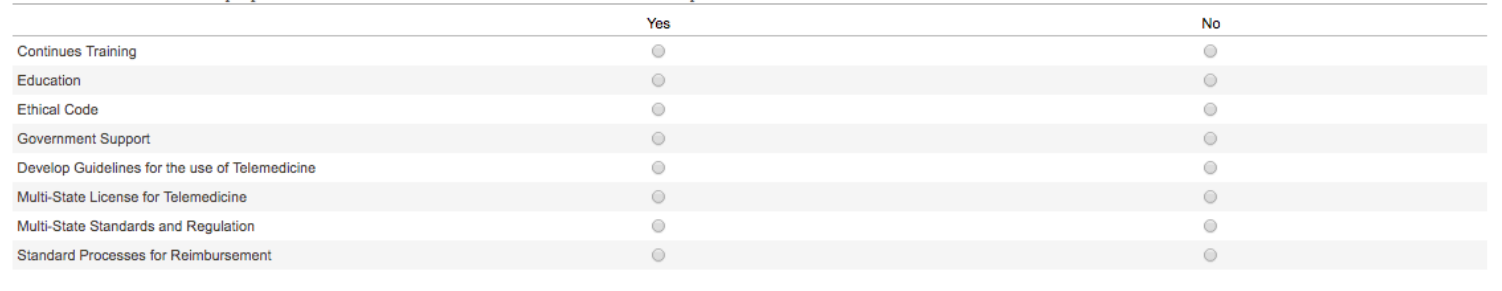

If you think there are other possible solutions not included in the model, please add them in the space below 


\section{Appendix D: Judgment Quantification Instrument}

\section{Instrument 1}

f Portland $\underset{\text { UniversirY }}{\text { State }}$

literature review reveals that there are several barriers impacting the adoption of teleconsultation through perceived usefulness and ease of use, which are the two components of the Technology Acceptance Model (TAM). Please click here to get more information about the Technology Acceptance Model (TAM).
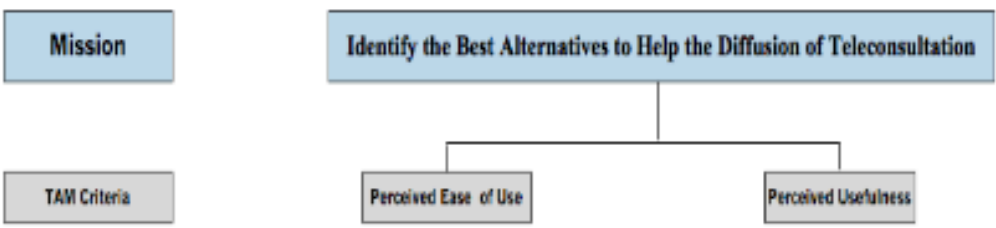

Please use the slider to determine the score of the first alternative (shown bold in red) compared to the second alternative with respect to the mission.

Please distribute 100 points between the following pairs of the components of the Technology Acceptance Model (TAM) perceived usefulness and ease of use to reflect your judgment on their relative importance to the mission.

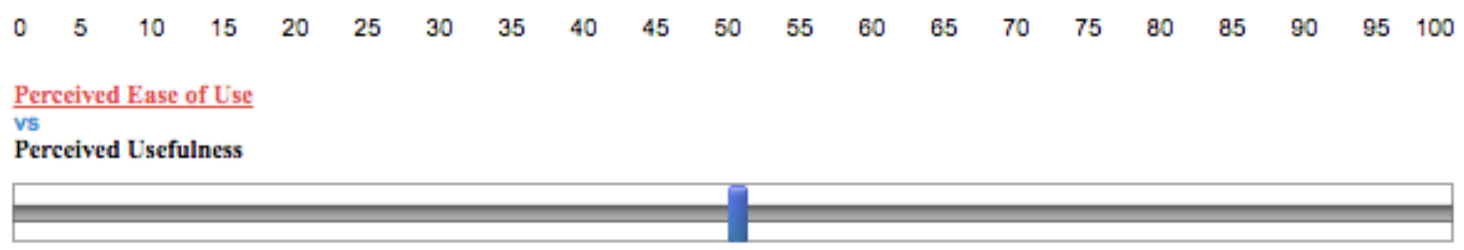




\section{Instrument 2}

面P Portland $\underset{\text { Universitr }}{\text { State }}$

Literature review reveals that there are four main barriers to the adoption of teleconsultation which are financial, technical, logistical, and cultural. Please click here for more information about each barier.

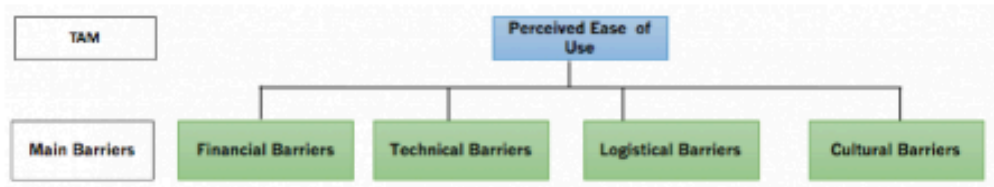

Please use the slider to determine the scare of the first aitemative (shown bold in red) compared to the second alternative with respect to the perceived ease of use.

Please distribute 100 points between the following pairs of the four main barrlers to reflect your judgment on their relative importance to the perceived ease of use.

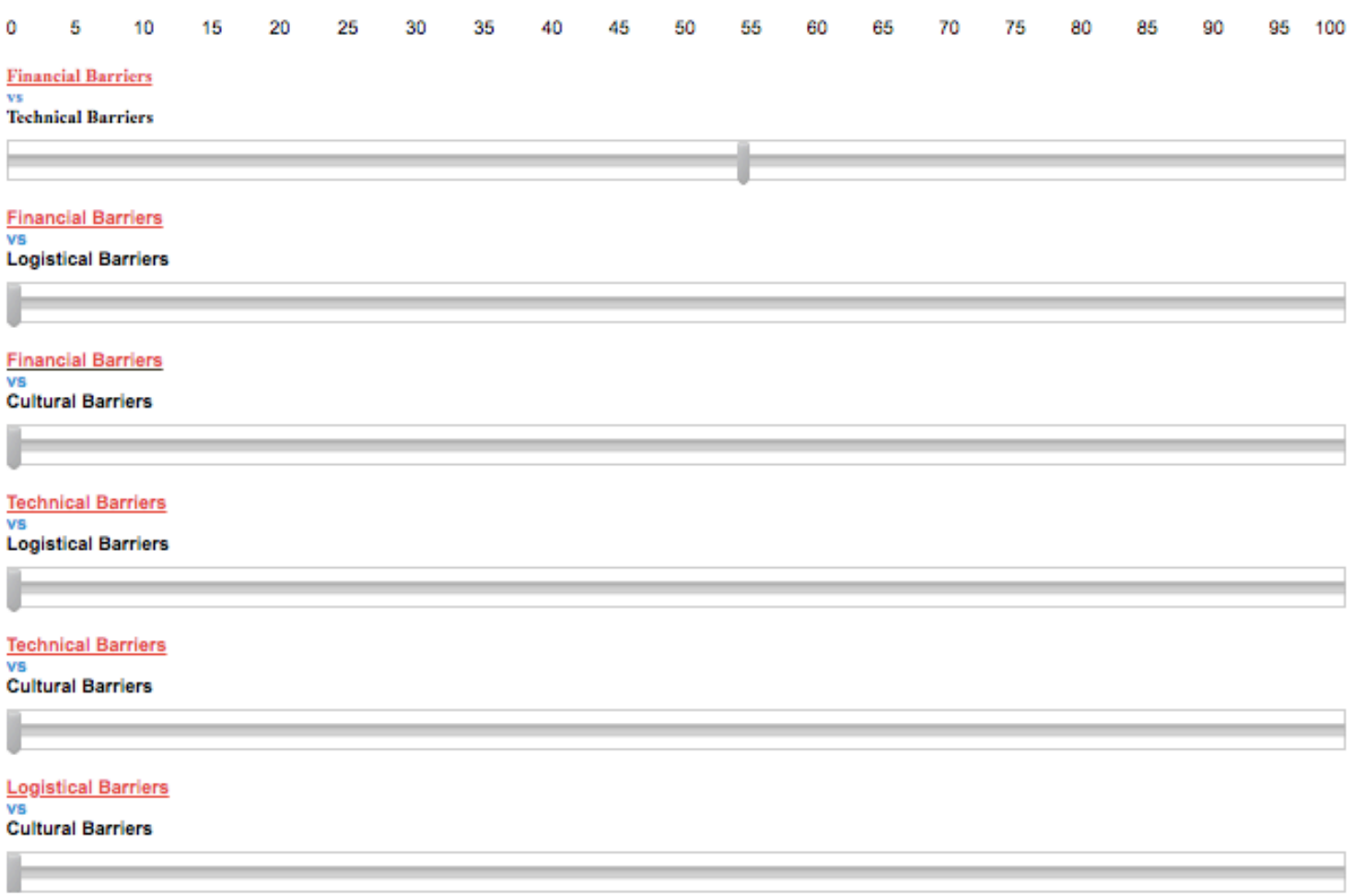


P Portland $\underset{\text { UNIVERITY }}{\operatorname{State}}$

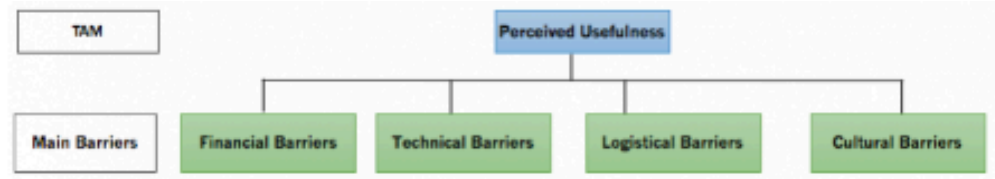

Please use the slider to determine the scare of the first altemetive (shown bold in red) compared to the second alternative with respect to the perceived usefuiness.

Please distribute 100 points between the following pairs of the four main barriers to reflect your judgment on their relative importance to the perceived usefuiness.

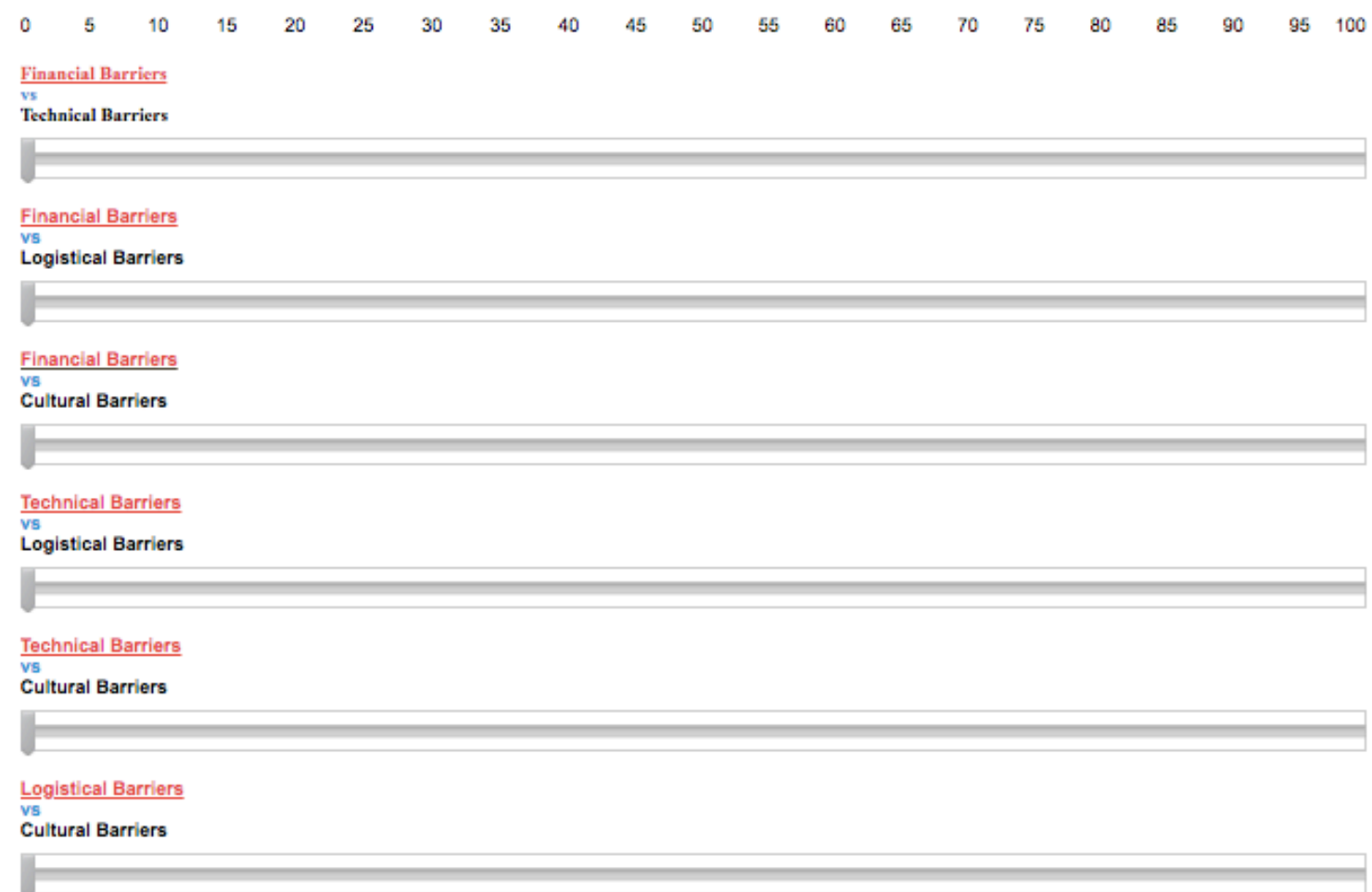




\section{Instrument 3}

P Portland State

The literature review reveals that there are two sub-barriers associated with financial barriers to the adoption of teleconsultation that are startup cost and ongoing cost. Please click here for more information about each sub-barrier.
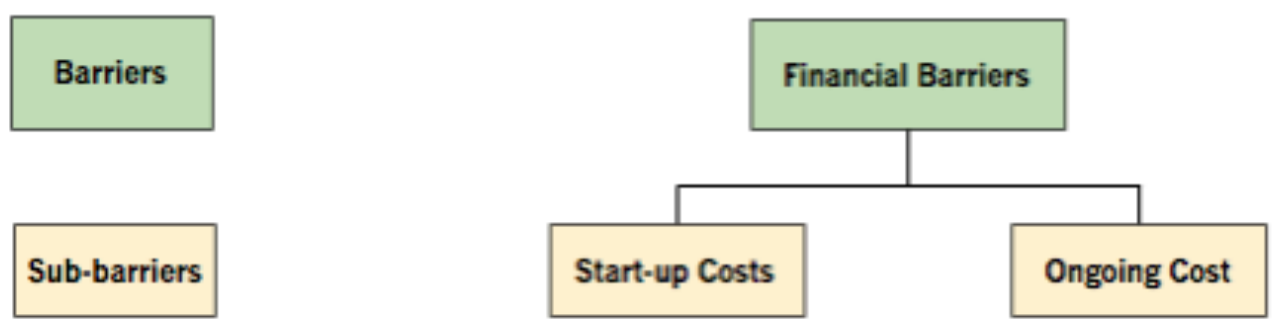

Please use the slider to determine the score of the first alternative (shown bold in red) compared to the second alternative with respect to the financial barriers.

Please distribute 100 points between the following pairs of sub-barriers to reflect your judgment on their relative importance to the financial barriers.

$\begin{array}{lllllllllllllllllllll}0 & 5 & 10 & 15 & 20 & 25 & 30 & 35 & 40 & 45 & 50 & 55 & 60 & 65 & 70 & 75 & 80 & 85 & 90 & 95 & 100\end{array}$

$\underline{\text { Start-up Costs }}$

vs

Ongoing Cost 


\section{Instrument 4}

面P Portland $\underset{\text { UNIVERSITY }}{\text { State }}$

The literature review reveals that there are five sub-barriers associated with technical barriers to the adoption of teleconsultation that are computer skills, training, technical support, infrastructure and privacy \& security. Please click here for more information about each sub-barrier.
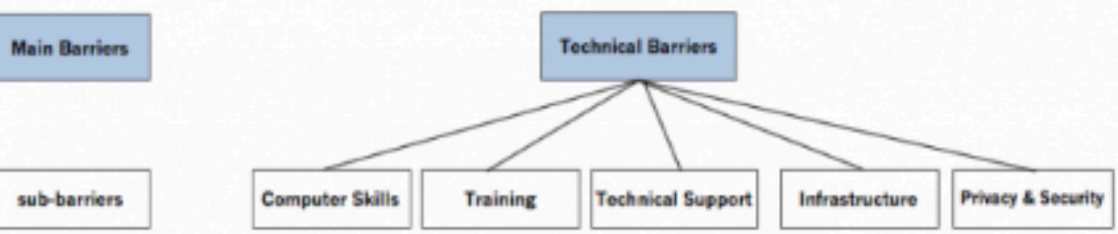

Please use the slider to determine the score of the first alternative (shown bold in red) compared to the second alternative with respect to the technical barriers.

Please distribute 100 points between the following pairs of sub-barriers to reflect your judgment on their relative importance to the technical barriers.

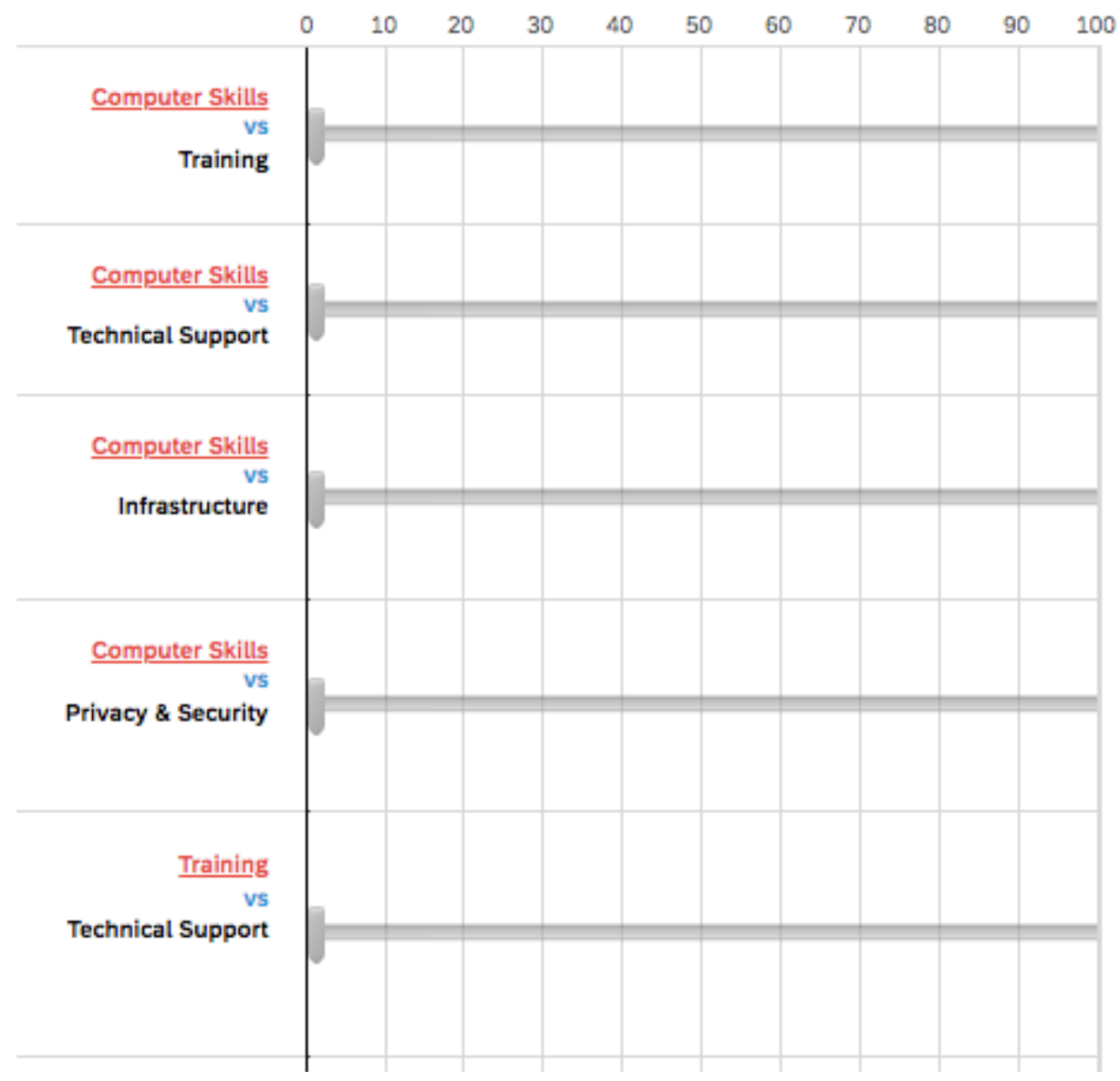




\section{Instrument 5}

面P Portland $\operatorname{UN}_{\text {NIVRSITY }}$

The literature review reveals that there are four sub-barriers associated with logistical barriers to the adoption of teleconsultation that are licensure, credentialing, malpractice and reimbursement. Please click here for more information about each sub-barrier.

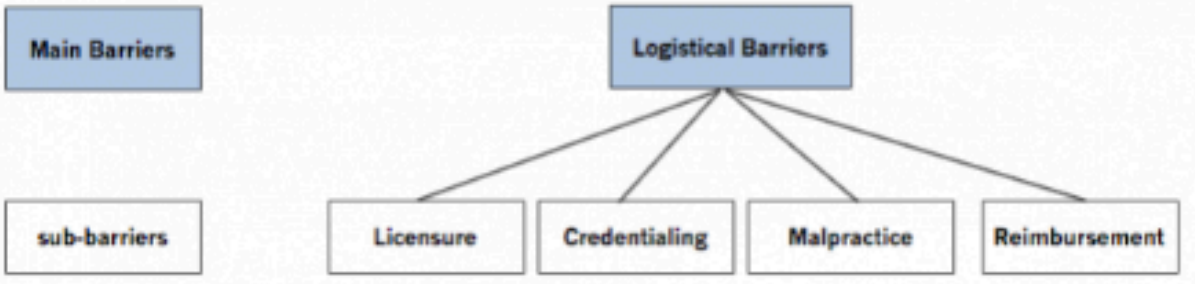

Please use the slider to determine the score of the first alternative (shown bold in red) compared to the second alternative with respect to the logistical barriers.

Please distribute 100 points between the following pairs of sub-barriers to reflect your judgment on their relative importance to the logistical barriers.

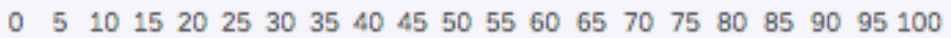

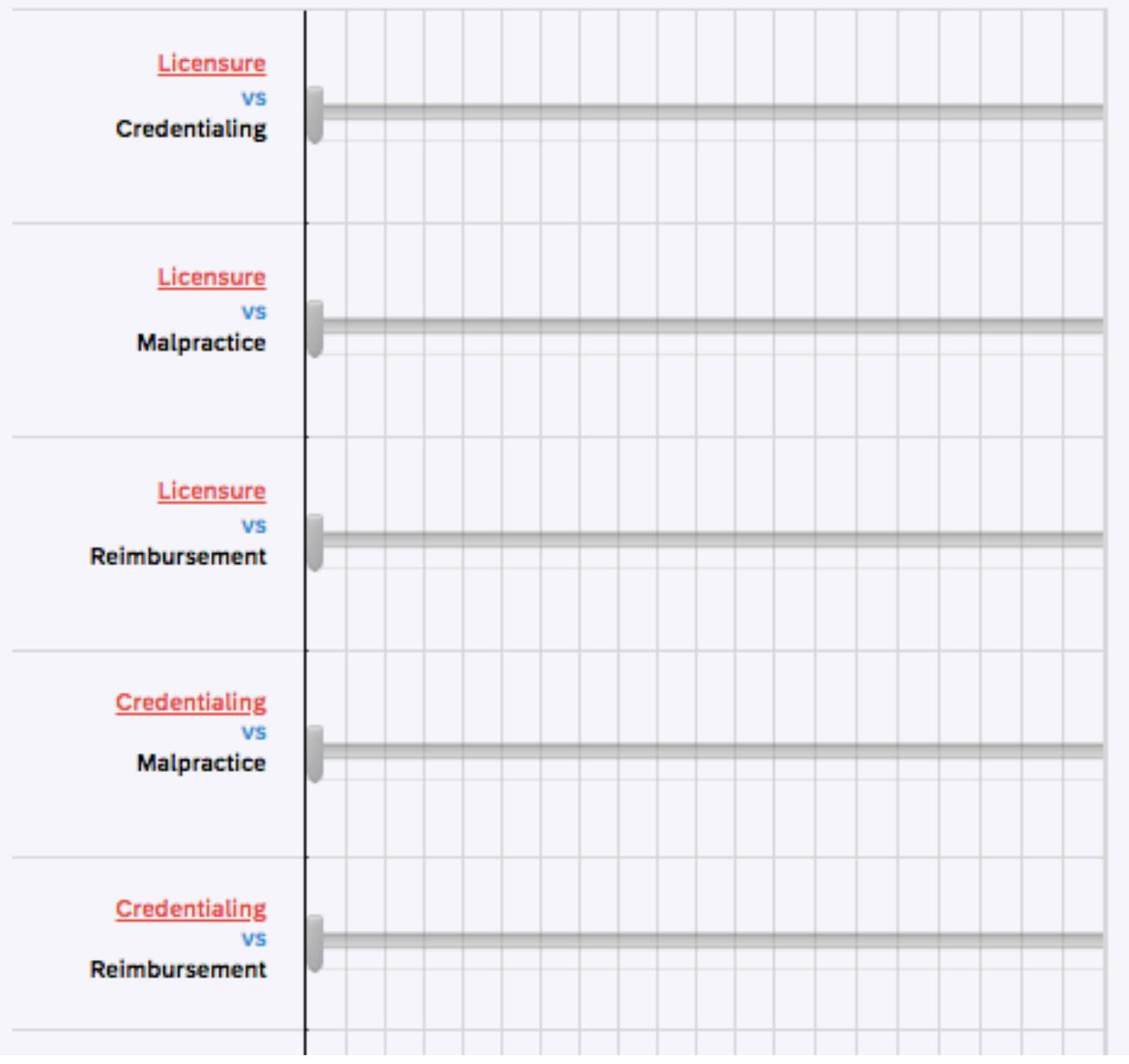




\section{Instrument 6}

面P Portland $\underset{\text { Universitr }}{\text { State }}$

The literature review reveals that there are four sub-barriers associated with cultural barriers to the adoption of teleconsultation that are physician acceptance, patient acceptance, and patient satisfaction. Please click here for more information about each sub-barrier.
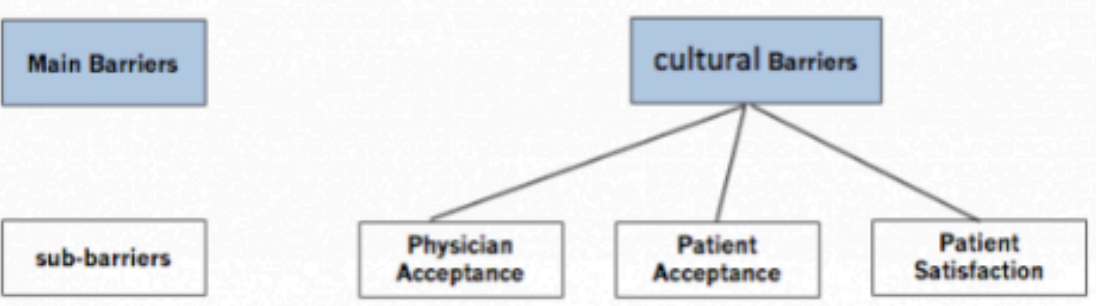

Please use the slider to determine the score of the first alternative (shown bold in red) compared to the second alternative with respect to the cultural barriers.

Please distribute 100 points between the following pairs of sub-barriers to reflect your judgment on their relative importance to the cultural barriers.

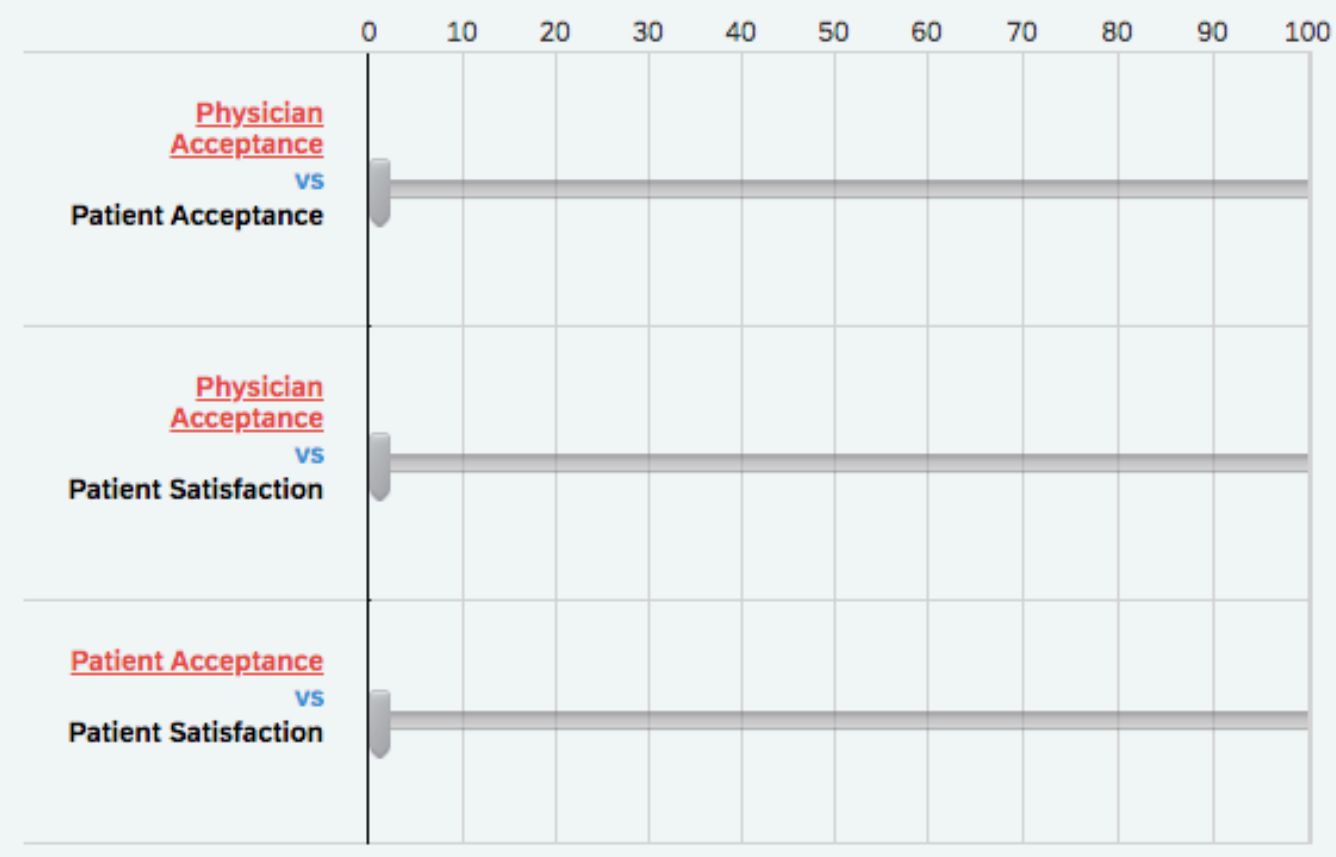




\section{Instrument 7}

面P Portland $\operatorname{UN}_{\text {NIVRSITY }}$

The literature review reveals that there are seven alternative solutions associated with the adoption of teleconsultation that are continues training, education, government support, develop guidelines for the use of teleconsultation, multi-state license for teleconsultation, multi-state standards and regulation and standard processes for reimbursement. Please click here for more information about each solution.

Please use the slider to determine the score of the first alternative (shown bold in red) compared to the second alternative with respect to the alternative solutions.

Please distribute 100 points between the following pairs of sub-barriers to reflect your judgment on their relative importance to the alternative solutions.

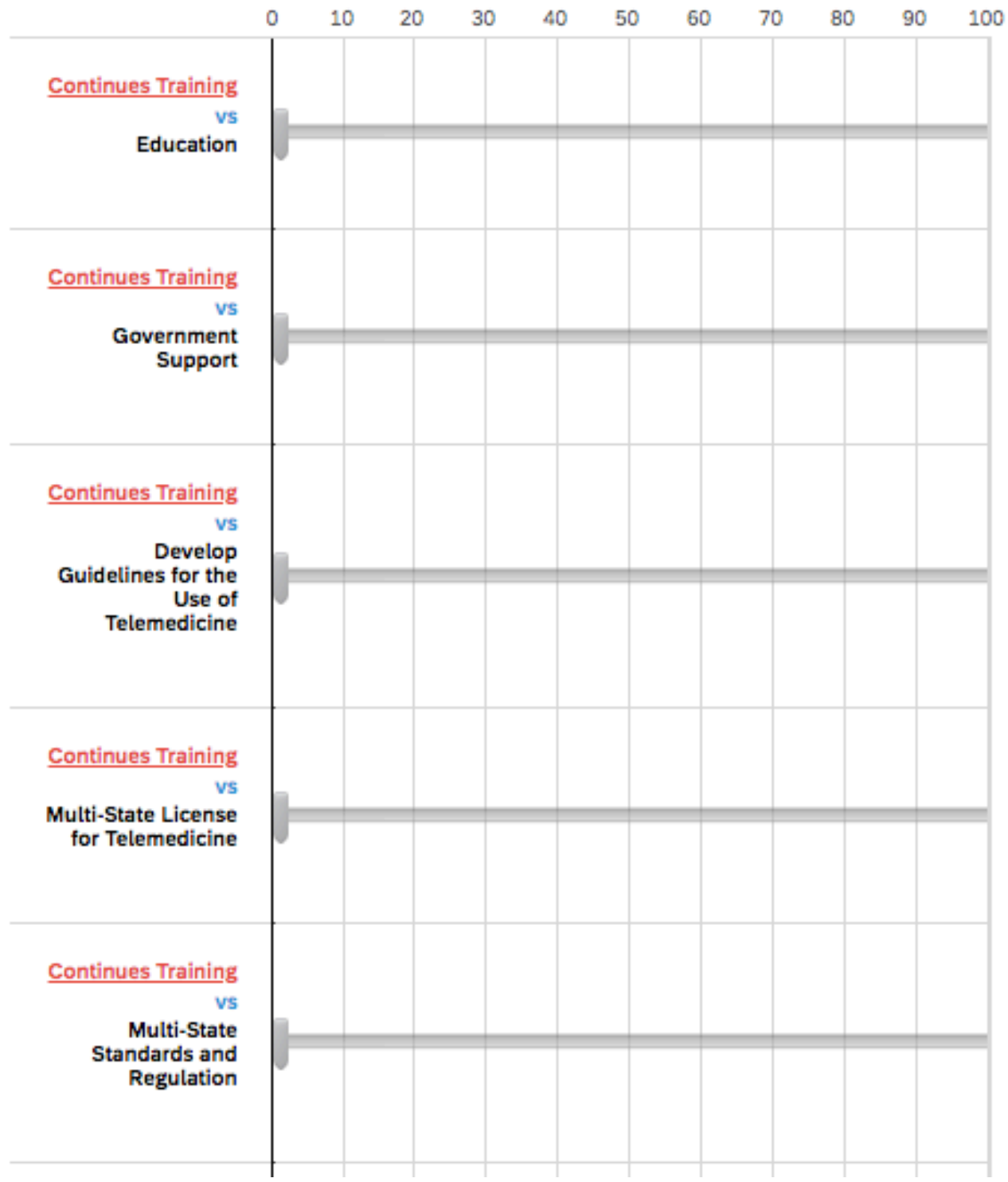

